Stochastic control and optimal stopping for non-Markov processes with infinite horizon and related topics

\title{
SVEN HAADEM
}

DisSERTATION PRESENTED FOR THE DEGREE of Philosophiae Doctor

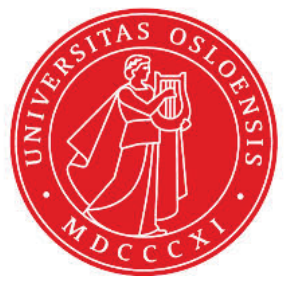

Department of MATHEMATiCs

UNIVERSITY OF OSLO

2013 
(C) Sven Haadem, 2014

Series of dissertations submitted to the

Faculty of Mathematics and Natural Sciences, University of Oslo No. 1465

ISSN $1501-7710$

All rights reserved. No part of this publication may be reproduced or transmitted, in any form or by any means, without permission.

Cover: Inger Sandved Anfinsen.

Printed in Norway: AIT Oslo AS.

Produced in co-operation with Akademika Publishing.

The thesis is produced by Akademika Publishing merely in connection with the thesis defence. Kindly direct all inquiries regarding the thesis to the copyright holder or the unit which grants the doctorate. 
I would like to dedicate this thesis to my loving wife, parents and sisters. 



\title{
Acknowledgements
}

\author{
"Rabbit's clever," said Pooh thoughtfully. "Yes," said Piglet, \\ "Rabbit's clever." "And he has Brain." "Yes," said Piglet, \\ "Rabbit has Brain." There was a long silence. "I suppose," \\ said Pooh, "that that's why he never understands \\ anything." - A.A. Milne, Winnie-the-Pooh.
}

To continuously develop and challenge oneself one needs to invest a great deal of will and energy. The life changing challenges need to be found and conquered. To do that successfully one needs to develop a deep self awareness. It takes courage and strength to find and ask the right questions.

It is hard to know when it is needed to go beyond the surface. Often the most elegant and simple things are the ones that requires the most thought. It is easy to feel comfortable with the obvious appearance. If one skips the deeper analysis one often miss the most valuable lessons life has to offer. So, it is of great importance, as I learned the hard way many times during my $\mathrm{PhD}$, to always be critical. With all things in life, don't let the shiny surface stop you from digging deeper. Invest the time and effort. Take for example the famous sentence that often pops up on small gift shop signs and in self-help books: "Be the change you wish to see in the world." Without reflecting on the context and broader statement it doesn't provide any insight and miss the depths in Gandhi's messages. It can easily be turned into a simple excuse. If so, it takes away much of our responsibilities and oversimplifies society. It states the most trivial part that I believe most people realize by themself but if you don't dig deeper the important part is missed. You also need to take responsibility for the change through hard work and courage. It appears so deep and elegant that you are easily tempted to swallow it without giving it any more thought.

For me, the first quote is the other way around. It appears simple but a little dumb at first. Then, the more you contemplate on it the deeper it grows. It summarize a feeling I always have had problems putting into words. It sometimes comes sneaking up on me when I am buried in a long chain of thoughts. Of course i don't say I don't understand anything as I have undoubtedly learned much during my 
PhD. The thing is, what I learned and value the most isn't the technicalities and mathematics. Rather it is how to apply these new ways of thinking in all areas of my life. I have learned to be critical, structure my thoughts and organize all complex system I stumble upon. This way, new wisdom and knowledge is easier to come by and my horizon expands faster and faster. This has allowed me to grow as a person but it comes at a cost. One gets caught up in complexities, get buried in thoughts and may miss out on valuable connections to other people.

Since the Norwegian society is so small with limited resources, we need to understand our strengths and focus on them. To be able to develop these strengths such that we can stay competitive in an increasingly interacting and competitive world, we need to attract talented people. The Norwegian system where you as a phd student is given a generous wage is a great step towards attracting these talents, both locally and internationally. Moreover, as a byproduct of not only attracting these talents locally, we receive a blend of cultures. This provides us with different viewpoints and innovations. All of this are factors that enables us to remain a society with a high living standard, high respect for diversity, acceptance and a relatively small difference between the rich and the poor.

It has been a long and windy road and without all the support from supervisors, family, friends and colleagues this thesis would never made it to the print. Even though it was frustrating at times, I am very glad I did take this road. I believe I will look back at this period as one of the most rewarding and important moments of my life.

Many big thanks from the deepest place in my heart to all who supported me in any way. I would like to mention a few, but the list is far from complete:

- Frank Proske - for his constant positivity and selflessness. Always able to keep me afloat, always a new perspective and never allowing negativity to get a hold. Whitout his selfless guidance I would never have made it to the end. I have learnt more from him than I ever could dream of, both mathematically and personally. A great inspiration and mentor. Without him this thesis would never have happened.

- Bernt Øksendal - for being a true inspiration and guide. Bernt has a way to always finding a solution when it seems the furthest away. Never judging and always treating others with grace and respect. A patient guide through the mathematical jungle and a very important part of this thesis. 
- S. R. Srinivasa Varadhan and Courant institute at New York University for allowing me to visit during the Spring of 2013. I was warmly welcomed and had a great challenging stay.

- My fellow Phd colleagues.

- Everyone at the math department and CMA for creating an exceptional working environment.

- My wife - not much mathematical guidance here, but on the other side more support and love than any man could ever dream of. Always pushing for challenges that I must overcome. Enabling me to grow and be a better person. My true soulmate.

- My family - unconditionally there. Supportive beyond the call of duty. Always helping me be the one I want to be.

- My friends - without them this thesis would have been finished years earlier.

Once more (something this important is worth repeating); thanks to anyone who was in anyway involved in this process. I appreciate all the support beyond any words.

I would like to finish with a quote that I find very insightful and that one should always keep in mind. When teaching, leading others, being lead or when judging politicians;

\section{"As I grow older, I pay less attention to what men say. I just watch what they do." - Andrew Carnegie.}





\begin{abstract}
In this thesis five articles are presented.

1. The first article is entitled "Optimal stopping and stochastic control differential games for jump diffusions" which was written with: Fouzia Baghery, Bernt Øksendal and Isabelle Turpin and published in Stochastics: An International Journal of Probability and Stochastic Processes. In this article we have an investor who wants to find the optimal time for selling the investment. Optimal is considered to be the same as risk minimal under some convex risk measure. The stochastic differential games under study are jump diffusions driven by Brownian motions and compensated Poisson random measures, where one of the players can choose the stochastic control and the other player can decide when to stop the system. Further, a verification theorem for such games in terms of a Hamilton-Jacobi-Bellman variational inequality (HJBVI) is proven. We also prove that the value function of the game is a viscosity solution of this associated HJBVI. The results are applied to study some specific examples, including optimal resource extraction in a worst case scenario, and risk minimizing optimal portfolio and stopping.

2. The second article is entitled "Maximum principles for jump diffusion processes with infinite horizon". It is written in collaboration with Bernt Øksendal and Frank Proske and published in Automatica. In this article we try to find a good extension of the maximum principle to infinite horizons and partial information. The results are applied to an optimal consumption and portfolio problem in infinite horizon.

3. The third article is entitled "A Maximum Principle for Infinite Horizon Delay Equations". It is written in collaboration with Nacira Agram, Bernt Øksendal and Frank Proske. The article is to be published in SIAM. In this article we continue to look at infinite horizons. Now, in a delay equation setting. We prove a maximum principle of optimal control of stochastic delay equations on infinite horizon. We establish first and second sufficient stochastic maximum principles as well as necessary conditions for that problem. We illustrate our results by an application on optimal consumption rate for an economic quantity.
\end{abstract}


4. The fourth article is entitled "Backward Stochastic fractional differential equations and a maximum principle with infinite horizon driven by a fractional Brownian motion". Continuing on with infinite horizon but changing the driver to a fractional Brownian motion. Using fractional Brownian motion makes the whole framework much more complex and the requirements in the theorems get stronger and more demanding. We show existence and uniqueness of backward stochastic differential equations (BSDEs) on infinite horizon driven by a fractional Brownian motion (fBm). Then we prove a maximum principle for the problem of optimal control for a fractional SDE with infinite horizon and partial information. Further, we prove existence of fractional backward stochastic differential equations on infinite horizon. We illustrate our findings by an example.

5. The fifth article is entitled "On the Construction and Malliavin Differentiability of Lévy Noise Driven SDEs with Singular Coefficients". It is written in collaboration with Frank Proske and is to be published in Journal of Functional Analysis. In this paper we consider solutions of SDEs driven by a Lévy process with non-smooth coefficients. In this paper we introduce a new technique to construct unique strong solutions of SDE's with singular coefficients driven by certain Lévy processes. Our method which is based on Malliavin calculus does not rely on a pathwise uniqueness argument. Furthermore, the approach, which provides a direct construction principle, grants the additional insight that the obtained solutions are Malliavin differentiable. 


\section{Contents}

1 Introduction

1.1 Optimal stopping and stochastic control differential games for jump diffusions . . . . . . . . . . . . . . 3

1.2 Maximum principles for jump diffusion processes with infinite horizon . . . . . . . . . . . . . 6

1.3 A Maximum Principle for Infinite Horizon Delay Equations . . . . . . . . . . . . . . . . . . 9

1.4 Backward Stochastic fractional differential equations and a maximum principle with infinite horizon driven by a fractional Brownian motion . . . . . . . . . . . . . . . . . . . . . . . 13

1.5 On the Construction and Malliavin Differentiability of Lévy Noise Driven SDEs with Singular Coefficients 18

\section{Articles 22}

2 Optimal Stopping and Stochastic Control 23

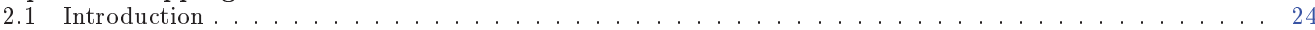

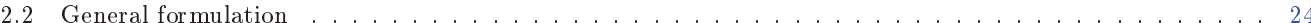

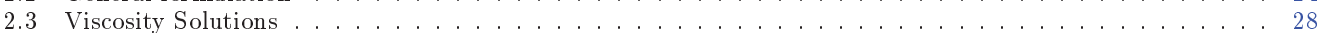

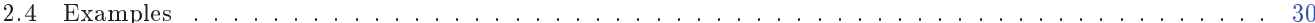

2.5 Proof of the Dynamical Programming Principle (Prop. 2.3) . . . . . . . . . . . . . . . . 38

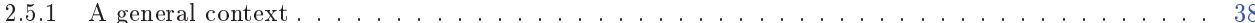

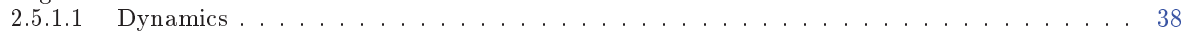

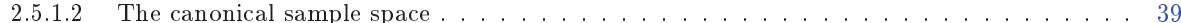

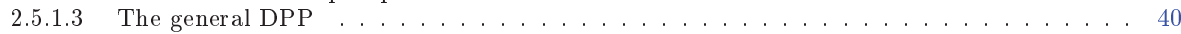

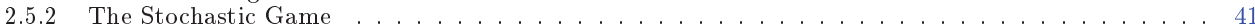

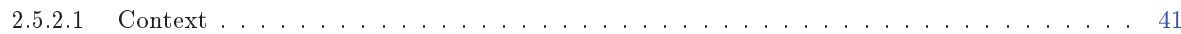

2.5.2.2 Admissible Controls and Strategies . . . . . . . . . . . . . . . . . . . . . . . . 41

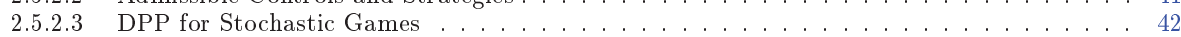

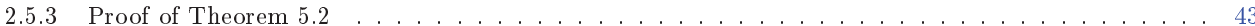

2.5.3.1 Intermediate Results . . . . . . . . . . . . . . . . . . . . 43

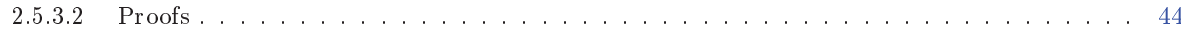

3 Infinite Horizon Maximum Principle 52

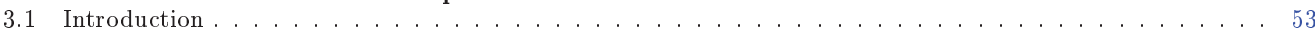

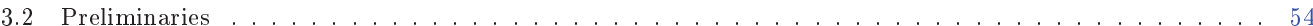

3.3 Existence and Uniqueness . . . . . . . . . . . . . . . . . . . . . . . . 55

3.4 Optimal control with partial information and infinite horizon . . . . . . . . . . . . . . . . . 60

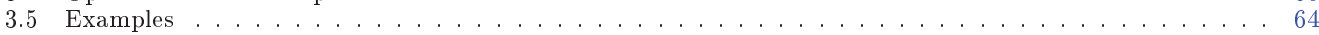

3.6 Necessary Maximum Principle . . . . . . . . . . . . . . . . . . . . . . . . . . . . . . . . 73

4 Infinite Horizon with Delay 77

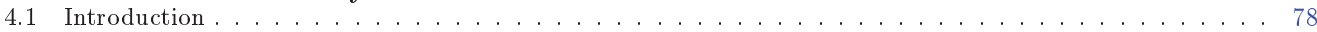

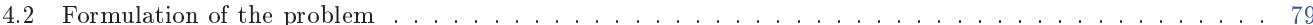

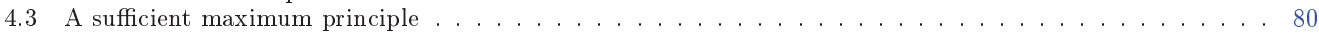

4.3.1 Hamiltonian and time-advanced BSDEs for adjoint equations . . . . . . . . . . . . . . . 80

4.3 .2 A first sufficient maximum principle . . . . . . . . . . . . . . . . . . . . 81

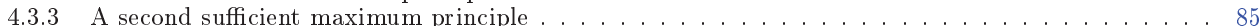

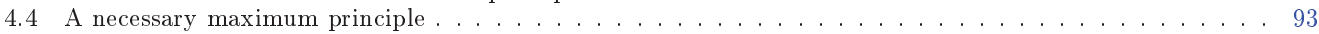

4.5 Existence and uniqueness of the time-advanced BSDEs on infinite horizon . . . . . . . . . . . . 96 
5 Fractional Brownian Motion 106

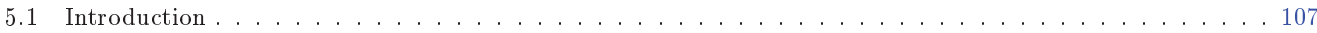

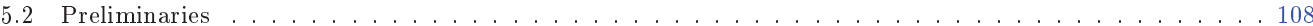

5.3 Fractional Wick-Itô-Skorohod integral in $L^{2} \ldots \ldots \ldots \ldots \ldots \ldots$

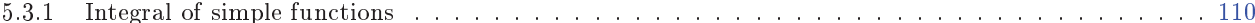

5.3 .2 FWIS integral for general stochastic functions . . . . . . . . . . . . . . . . . 111

5.3 .3 Itô's formula . . . . . . . . . . . . . . . . . . . . . . . . . . . . 113

5.3.4 Fractional Clark-Haussmann-Ocone theorem . . . . . . . . . . . . . . . . . . 113

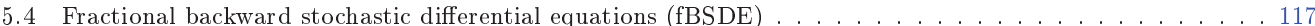

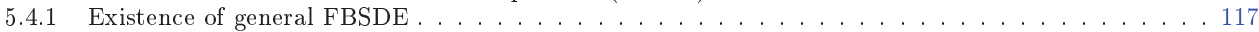

5.4 .2 Linear infinite horizon backward stochastic differential equations (LIH-BSDE) . . . . . . . 123

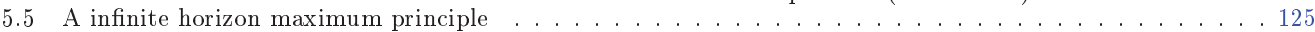

6 SDEs with Singular Coefficients 131

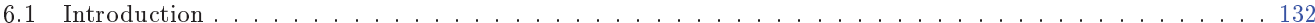

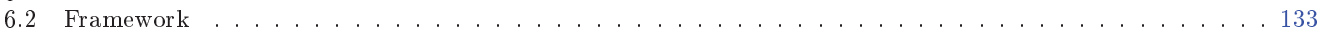

6.2 .1 Hölder Spaces . . . . . . . . . . . . . . . . . . . . . . . . . . . . . . . . 133

6.2 .2 Lévy Processes . . . . . . . . . . . . . . . . . . . . . . . . . . . . . 134

6.2.3 Chaos Expansions and the Malliavin Derivative . . . . . . . . . . . . . . . . . . . . 135

6.2 .4 Fractional Sobolev Spaces . . . . . . . . . . . . . . . . . . . . . 137

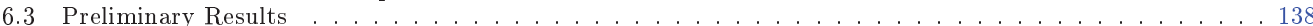

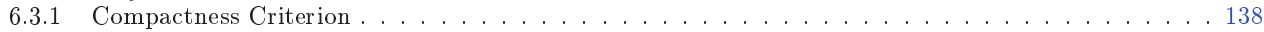

6.3.2 Some Regularity Results . . . . . . . . 140

6.3.2.1 Kolmogorov Type Equations Associated with Lévy Processes . . . . . . . . . . . . . . . . 140

6.4 Construction of Solutions to SDE's via the Compactness Criterion for $L^{2}(\mu) \ldots \ldots \ldots 1$

\section{Future Work}

7 Future Work 162

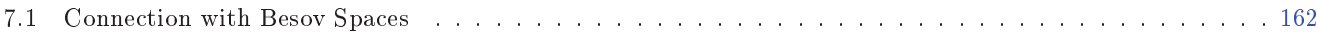

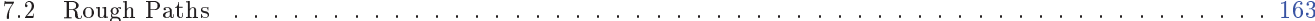

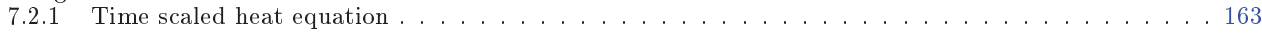

\section{Background Material 172}

8 Basic Stochastic Analysis 173

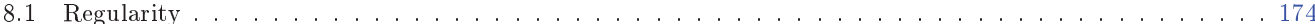

8.2 Convergence and Local Martingales . . . . . . . . . . . . . . . . . . . . . . . . 175

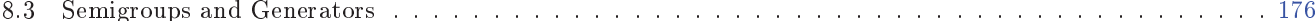

8.4 Integration with Martingales and Semi-Martingales . . . . . . . . . . . . . . . . . 177

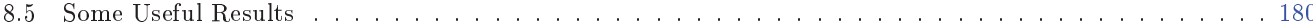

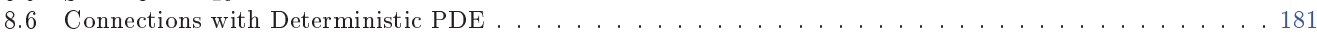

9 Lévy Processes

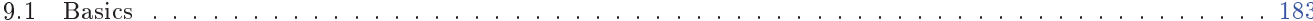

9.2 Semigroups and Generators . . . . . . . . . . . . . . . . . . . . . . . . 184

9.3 Stochastic Integration . . . . . . . . . . . . . . . . . . . . . . . . . . . 185

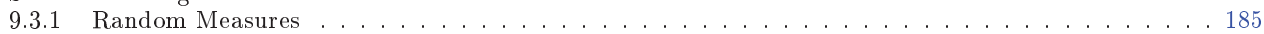

10 Optimal Control 187

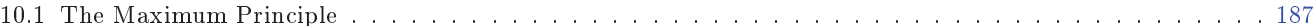

10.1.1 A General Maximum Principle for Brownian Motions . . . . . . . . . . . . . . . . . . 187

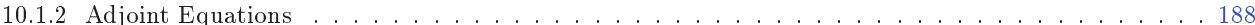

10.1.3 A Concave Maximum Principle for Jump Diffusions . . . . . . . . . . . . . . . . . . 190

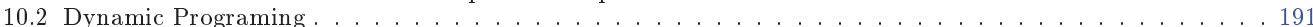

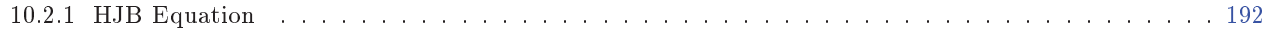

10.2 .2 Viscosity Solution . . . . . . . . . . . . . . . . . . . . . . . . . . . . . . . . . . . . . . . .

10.2 .3 Jump Diffusions . . . . . . . . . . . . . . . . . . . . . . . . . . . . . . 193

10.3 Connection Between the Maximum Principle and Dynamic Programing . . . . . . . . . . . . . . . . 194

10.3.1 Relationship for Deterministic Systems . . . . . . . . . . . . . . . . . . . . . 194

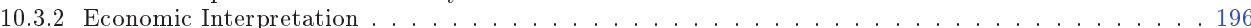

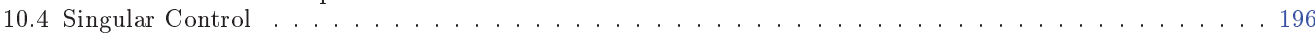

11 Optimal Stopping 199

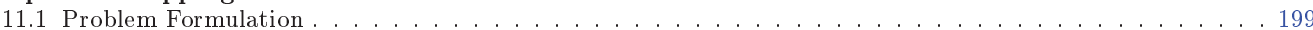

11.2 Sufficient Optimal Stopping Theorem . . . . . . . . . . . . . . . . . . . . . . . 200 
12 Risk Measures 201

12.1 Value at Risk . . . . . . . . . . . . . . . . . . . . . . . . . . 201

12.2 Coherent Risk Measures . . . . . . . . . . . . . . . . . . . . . . . . . . . . . . 202

12.3 An Axiomatic Approach . . . . . . . . . . . . . . . . . . . . . . . 203

12.3.1 Axioms on Acceptance Sets . . . . . . . . . . . . . . . . . . . . . . . . 205

12.4 A Generalization to Convex Risk Measures . . . . . . . . . . . . . . . . . . . . . . . 206

12.5 g-Brownian Motion and Dynamic Risk Measures . . . . . . . . . . . . . . . . . . . . . . . . . . 208

12.5.1 Dynamic Risk Measures from g-Expectations . . . . . . . . . . . . . . . . . . . . . 209

12.6 G-Brownian motion . . . . . . . . . . . . . . . . . . . . . . . . . 209

13 Stochastic Differential Equations 211

13.1 Strong Solutions . . . . . . . . . . . . . . . . . . . . . . . . . . 212

13.2 Weak Solutions . . . . . . . . . . . . . . . . . . . . . . . . . . . . . . . 212

13.3 Stochastic Differential Equations for Lévy Processes . . . . . . . . . . . . . . . . . . . . . 214

13.4 Stochastic Flows and Cocycles . . . . . . . . . . . . . . . . . . . . . . . . . . . 215

14 Backward Stochastic Differential Equations 216

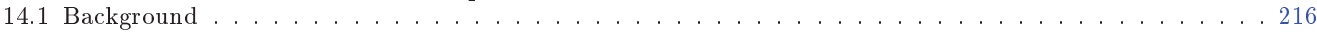

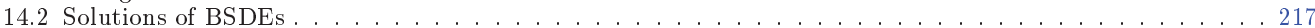

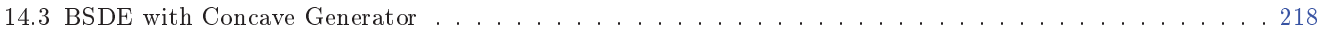

14.4 Forward-Backward Stochastic Differential Equations . . . . . . . . . . . . . . . . . . . . . . 218

14.4.1 Backward Stochastic Differential Equations and g-Expectations . . . . . . . . . . . . . . . . 219

15 Dirichlet Forms

15.1 Unbound Operators in Banach Spaces . . . . . . . . . . . . . . . . . . . . . . . . . . 220

15.1 .1 Closed symmetric forms . . . . . . . . . . . . . . . . . . . . . . . . . 221

15.1.2 Fourier Transform and Pseudo-Differential Operator . . . . . . . . . . . . . . . . . . . . 221

15.2 Closed Forms and Semigroups . . . . . . . . . . . . . . . . . . . . . . . . . 222

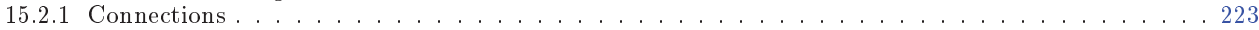

16 Delay Equations

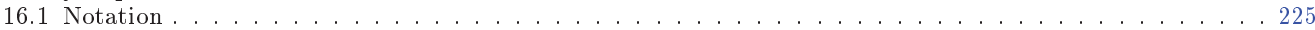

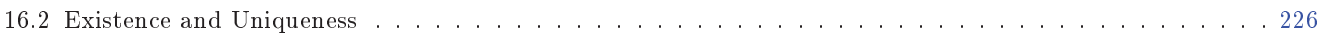

16.3 Markov Property . . . . . . . . . . . . . . . . . . . . . . . . . . . . . . . . . . . . . . . . . .

17 Besov Spaces 228

18 Rough Paths

18.1 Hölder and Sobolev Spaces . . . . . . . . . . . . . . . . . . . . . . . . 230

$18.2 p$-Variation and Hölder Continuous Paths . . . . . . . . . . . . . . . . . . . . . . . . 231

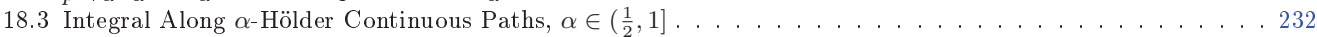

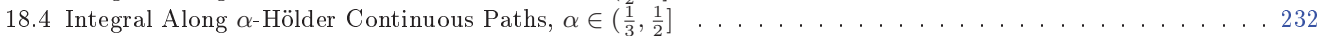

18.4.1 Construction of the Integral by Approximations . . . . . . . . . . . . . . . . . . . . . . 232

18.5 Rough Path Differential Equations . . . . . . . . . . . . . . . . . . . . . 235

19 Chaos Expansions and the Malliavin Derivative 236

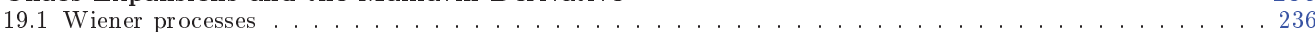

19.1.1 Hermite Polynomial, Function and the Wiener Chaos . . . . . . . . . . . . . . . 236

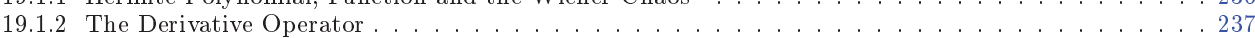

19.1 .3 The Divergence Operator . . . . . . . . . . . . . . . . . . . . . . . . . 238

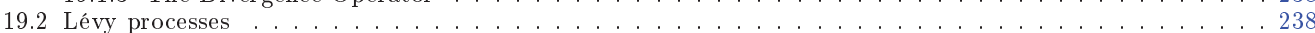

19.2.1 Malliavin Derivative for Lévy processes . . . . . . . . . . . . . . . . . . . . . 238

20 White Noise 240

20.1 Topological Vector Spaces . . . . . . . . . . . . . . . . . . . . . . . . . . . . . 240

20.2 White Noise Space . . . . . . . . . . . . . . . . . . . . . . . . . . . . . . . 241

20.2 .1 Construction of the White Noise Space . . . . . . . . . . . . . . . . . . . . . 242

20.3 Wick product . . . . . . . . . . . . . . . . . . . . . . . . . . . . . 243

20.4 Differential operators . . . . . . . . . . . . . . . . . . . . . . . . . . . . . . . . . . . . . . . . . . . . . . 244

21 Fractional Derivatives and Integrals 246

21.1 Riemann-Liouville Fractional Integrals . . . . . . . . . . . . . . . . . . . . . . . . . . . 247

21.2 Fractional Integrals of Weyls Type . . . . . . . . . . . . . . . . . . . . . . . 247

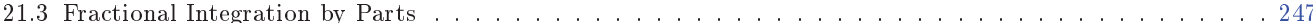

21.4 Riemann-Liouville Fractional Derivatives . . . . . . . . . . . . . . . . . . . . . . . . 247

21.5 Fractional Derivatives of Marchaud's Type . . . . . . . . . . . . . . . . . . . . . . . . . . 248 
22 Fractional Brownian Motion 249

22.1 Basics . . . . . . . . . . . . . . . . . . . . . . . . . . . . 249

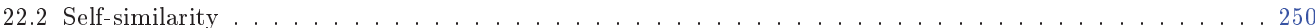

22.2 .1 Hölder and Martingale properties . . . . . . . . . . . . . . . . . 250

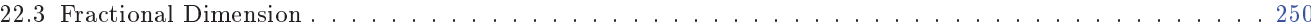

22.4 Moving Average Representation . . . . . . . . . . . . . . . . . . . . . . 250

22.5 Representation using Calculus of Variations . . . . . . . . . . . . . . . . . . . . . . . 251

22.6 Representation using White Noise . . . . . . . . . . . . . . . . . . . . . . 252

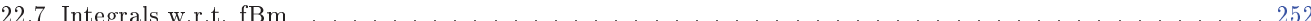

22.7 .1 Integrals w.r.t. $\mathrm{fBm}$ using White Noise . . . . . . . . . . . . . . . . 252

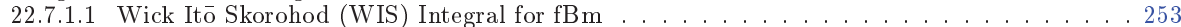

22.7.1.2 Fractional Wick Itō Skorohod (fWIS) integrals for fBm with Hurst index $H>\frac{1}{2} \ldots \ldots 254$

22.7.2 Integrals w.r.t. fBm using Youngs Integral . . . . . . . . . . . . . . . . . . . . 256

22.7 .3 Integrals w.r.t. fBm using Rough Paths . . . . . . . . . . . . . . . . . . 257

23 Banach Valued Processes 258

23.1 Spatially Homogeneous Noise and Random Fields . . . . . . . . . . . . . . . . . . . . . . . . . 258

23.1 .1 Walsh Method ... . . . . . . . . . . . . . . . . . . . 259

23.1 .2 Connection between the two integrals . . . . . . . . . . . . . . . . . . . . 260

23.2 Banach Spaces . . . . . . . . . . . . . . . . . . . . . . . 260

23.2 .1 Gaussian Measures . . . . . . . . . . . . . . . . . . . . . . . . . . . 261

23.2 .2 Reproducing Kernel Hilbert Space . . . . . . . . . . . . . . . . . . . . . . 262

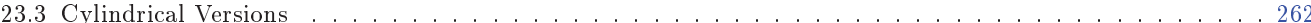

23.3 .1 Cylindrical Measures . . . . . . . . . . . . . . . . . . . . . . . . 262

23.3.2 Cylindrical Random Variables . . . . . . . . . . . . . . . . . . . . . . . . . . . . . . 263

23.3 .3 Radonification . . . . . . . . . . . . . . . . . . . . . . . 263

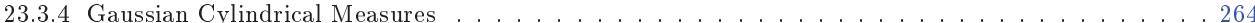

23.3 .5 -Operators . . . . . . . . . . . . . . . . . . . . . . . 264

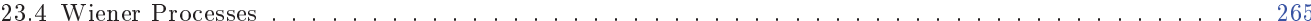

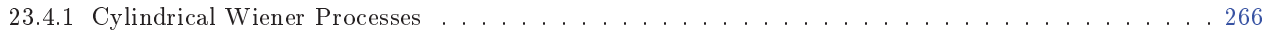

23.4.2 Cylindrical Stochastic Integral _. . . . . . . . . . . . . . . . . . . . . . . . 267

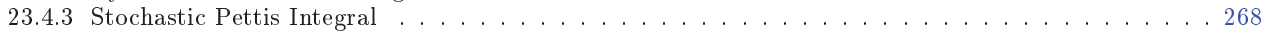

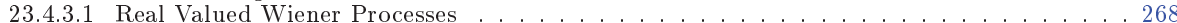

23.4.3.2 Cylindrical Wiener Processes . . . . . . . . . . . . . . . . . . . . 269

23.5 The Hilbert case ......... . . . . . . 271

23.5.1 Nuclear and Hilbert-Schmidt Operators . . . . . . . . . . . . . . . . . . . . 271

23.5.2 Hilbert-valued Wiener Processes . . . . . . . . . . . . . . . . . . . . . . . . . 271

23.6 H-valued Integrals . . . . . . . . . . . . . . . . . . . . . . . . . . . . . . . . . 272

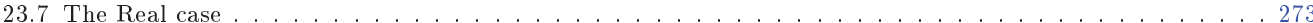

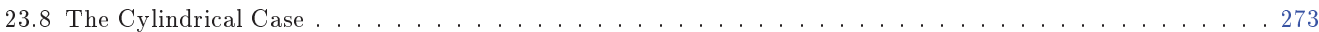

23.9 Relation with the Random Field Approach . . . . . . . . . . . . . . . . . . . . . . . . . . . 274

23.10Lèvy Processes in Banach spaces . . . . . . . . . . . . . . . . . . . . . . . . 275

24 Manifolds and Lie Groups 277

24.1 Matrix Groups $\ldots \ldots \ldots \ldots \ldots \ldots \ldots \ldots \ldots$

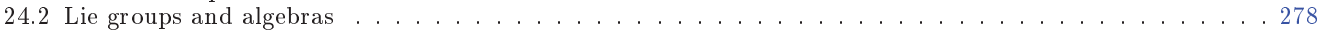

24.3 Martingales on Manifolds and Lie groups . . . . . . . . . . . . . . . . . . . . . . . . 278

References 


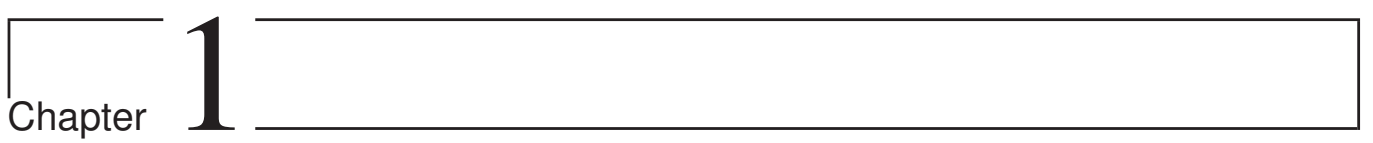

\section{Introduction}

Stochastic analysis is an important mathematical field that has proven very valuable both theoretically and in practical applications. It is a rich and rewarding subfield of mathematics for those who take the time to get to know it. Within stochastic analysis there are a large and fast growing counterpart to deterministic optimization, namely stochastic optimization. It is a realistic and useful extension of the deterministic theory and has countless real world applications.

Two large and important subfields of stochastic optimization is stochastic control and optimal stopping. Optimal stopping is concerned with the problem of choosing a time to take a particular action in order to maximise an expected reward (or equivalently, minimise an expected cost) in a stochastic setting. On the other hand, optimal control deals with the behavior of stochastic dynamical systems with inputs. These inputs needs to be controlled in an optimal way so that, as with optimal stopping, one maximises an expected reward.

There are generally two approaches of solving optimal stopping problems. One solution technique is the martingale approach, so called because it uses martingale theory, the most important concept being the Snell envelope. As optimal stopping problems often can often be written in the form of a Bellman equation, the problem can be solved by dynamic programming.

The two principal approaches to optimal control problems are the Pontryagin's maximum principle and Bellman's dynamic programming. The Pontryagin principle was formulated in 1956 by the Russian mathematician Lev Semenovich Pontryagin. The Euler-Lagrange equation of the calculus of variations is a special case of this principle. The principle states informally that a function called the Hamiltonian must be minimized over $\mathcal{U}$, the set of all permissible controls. Separately and independently Richard Bellman discovered a necessary condition for optimality. This method has become known as Bellman's dynamic programming. The point with the dynamic programming method is to breaks the decision problem into smaller subproblems. Start by considering the first decision separately, setting aside all future decisions. Then solve next subproblem until a solution of the original problem is found.

For finite horizon optimization problems one need to decide what happens when the horizon is reached. So, it is often preferable to look at infinite horizons to avoid the horizon problem. Infinite-horizon optimal control problems arise in many fields of economics, in particular in models of economic growth. Important examples are the perpetual American option and sustainability issues in optimal resource extraction. When it comes to stochastic control, the possibly infinite horizon does not cause any problems when using dynamic programming. However, dynamic programming can only be used for Markovian models. In non-Markovian models the maximum principle need to be used instead.

In the deterministic case the maximum principle by Pontryagin has been extended to infinitehorizon problems, but transversality conditions have not been given in general. The 'natural' transversality condition in the infinite case would be a zero limit condition, meaning in the economic sense that one more unit of good at the limit gives no additional value. But this 
property is not necessarily verified. In fact Halkin [1974] provides a counterexample for a 'natural' extension of the finite-horizon transversality conditions. Thus some care is needed in the infinite horizon case, and it is emphasize that the maximum principle for infinite horizon control is not a straightforward extension of the finite horizon case.

While stochastic optimization was in the beginning mainly studied in the context of Brownian motion a more realistic model is to take into account possible discontinuities. Thus a Lévy model is needed. This extension to include jumps gives us a much more realistic view on many real world problems.

Since dynamic system often have some kind of delay an extension to delay equations is of great importance. To this end we will explore a problem with delay differential equations (DDEs, or more generally functional differential equations, FDEs). A delay differential equation is a differential equation where the time derivatives at the current time depend on the solution and possibly its derivatives at previous times. While DDEs look a lot like ODEs the theory is more complicated and there are some surprising differences from ODEs. The study of infinite horizon delay equations has great real world applications.

Often it is useful to drop the requirement of independent increments for the Brownian motion. One way to do this is to consider a generalization of Brownian motion called fractional Brownian motion, $B^{H}(t)$. The fractional Brownian motion has fractional dimension. That is with probability 1 , the graph of $B^{H}(t)$ has both Hausdorff dimension and box dimension of $2-H$. When considering dynamic system based on fractional Brownian motion things get complicated very quickly. This is mainly due to the missing properties of Lévy processes like the Markov and Martingale properties. We often end up with much more complicated equations. But it may be worth it considering the more accurate description it gives of some real world systems, for example several financial markets. For $H>\frac{1}{2}, B^{H}$ has some amount of memory and may give a better model for some financial instruments like weather derivatives. The issue with fBm in financial markets and completeness is explored by Cheridito [2003]. If one assume the existence of an arbitrarily small minimal amount of time between transactions then there doesn't exist any arbitrage opportunities. However as C.Bender et al. [2006] points out this removes the possibility of continuous adjustments of the replication portfolio and thus hedging and replication may no longer be available.

To construct an infinite horizon maximum principle a solution of an infinite horizon backward stochastic differential equation (BSDE) is required. The problem then becomes that it requires a finite terminal time for the associated backward stochastic differential equation (BSDE). Thus, if the terminal time $T$ is infinite, what is the correct substitute for the terminal value of the solution of the BSDE? It seems that requiring a limit inequality on the terminal condition is the way to process. This approach seems to be in line with deterministic theory.

Differential equations play an important part in optimization. We need to know when we have a solution to our equations and the properties of the solution. Therefore we study the basic building block for the above: stochastic differential equations (SDEs). Consider the stochastic differential equation

$$
X_{t}=x+\int_{0}^{t} b\left(s, X_{s}\right) d s+L_{t}, 0 \leq t \leq T, x \in \mathbb{R}^{d} .
$$

Using Picard iteration it is well known that there exists a unique square integrable strong solution if the drift coefficient $b$ is Lipschitz continuous and of linear growth. However, what happens when $b$ is singular in the sense that $b$ is bounded and $\alpha$-Hölder continuous?

The analysis of strong solutions of SDEs with singular or non-Lipschitz coefficients is important and has been of interest for decades. Such solutions naturally arise from a variety of applications in the theory of controlled diffusion processes. For a singular function and Wiener process the solution has been intensively studied in the literature. A milestone in theory of 
SDEs is a result due to A.K. Zvonkin, Zvonkin [1974], who constructed unique strong solutions for Wiener process driven SDEs on the real line, when $b$ is merely bounded and measurable by employing estimates of solutions of parabolic partial differential equations and a pathwise uniqueness argument. Using similar techniques the latter result was subsequently extended to the multidimensional case.If the the driving process $L_{t}$ is a pure jump Lévy process we observe major differences to the Gaussian case. For example, if $L_{t}$ is a one-dimensional symmetric $\alpha$-stable process for $0<\alpha<1$ then one can find a bounded $\gamma$-Hölder-continuous drift coefficient $b$ with $\alpha+\gamma<1$ such that pathwise uniqueness of solutions to the SDE fails.

There are a follow up article where the solutions are in Hilbert spaces, but this article will not be included in this thesis.

As I want this thesis to be readable with just a basic understanding of stochastics, I have included a part which will hopefully guide a reader who is relatively new to stochastics to an understanding of the mathematics needed to read the articles. There is a small hope that the background notes may one day be a complete introduction to stochastic analysis. I also hope that in their present state they are able to give some insight. Enabling the reader both to read the articles and get a taste of the rich world of stochastic analysis and the deep roots to other mathematical subjects.

I will now try to provide a brief and and as non-technical as possible overview of all the articles and their main ideas.

\subsection{Optimal stopping and stochastic control differential games for jump diffusions}

The first article is: "Optimal stopping and stochastic control differential games for jump diffusions" which was written with Fouzia Baghery, Bernt Øksendal and Isabelle Turpin and published in Stochastics: An International Journal of Probability and Stochastic Processes.

Let $X(t)=X(t, \omega) \in[0, \infty) \times \Omega$ be a stochastic process on a filtered probability space $\left(\Omega, \mathcal{F},\left(\mathcal{F}_{t}\right)_{t>0}, \mathbb{P}\right)$. This process represents the wealth of an investment at time $t$ and the owner of the investment wants to find the optimal time for selling the investment. We let "optimal" be in the sense of "risk minimal", so that the problem is to find a stopping time $\tau=\tau(\omega)$ which minimizes $\rho(X(\tau))$, where $\rho$ denotes a risk measure (see chapter 12 on risk measures). If the risk measure $\rho$ is chosen to be a convex risk measure in the sense of Föllmer and Schied [2002] and (or) Frittelli and Gianin [2002], then it can be given the representation

$$
\rho(X)=\sup _{\mathbb{Q} \in \mathcal{N}}\left\{\mathbb{E}_{\mathbb{Q}}[-X]-\zeta(Q)\right\},
$$

for some set $\mathcal{N}$ of probability measures $\mathbb{Q} \ll \mathbb{P}$ and some convex "penalty" function $\zeta: \mathcal{N} \rightarrow \mathbb{R}$. Using this representation the optimal stopping problem above takes the form

$$
\inf _{\tau \in \mathcal{T}}\left(\sup _{\mathbb{Q} \in \mathcal{N}}\left\{\mathbb{E}_{\mathbb{Q}}[-X(\tau)]-\zeta(Q)\right\}\right)
$$

where $\mathcal{T}$ is a given family of admissible $\mathcal{F}_{t^{-}}$stopping times. This may be regarded as an optimal stopping-stochastic control differential game.

We till study this problem in a jump diffusion context. So, suppose now that we have a jump process and that the state $Y(t)=Y^{u}(t)=Y_{t}^{y, u}$ at time $t$ is given as the solution of a 
stochastic differential equation of the form

$$
\left\{\begin{array}{l}
d Y(t)=b\left(Y(t), u_{0}(t)\right) d t+\sigma\left(Y(t), u_{0}(t)\right) d B(t) \\
+\int_{\mathbb{R}_{0}^{k}} \gamma\left(Y\left(t^{-}\right), u_{1}(t, z), z\right) \tilde{N}(d t, d z) \\
Y(0)=y \in \mathbb{R}^{k}
\end{array}\right.
$$

Here $b: \mathbb{R}^{k} \times K \rightarrow \mathbb{R}^{k}, \sigma: \mathbb{R}^{k} \times K \rightarrow \mathbb{R}^{k \times k}$ and $\gamma: \mathbb{R}^{k} \times K \times \mathbb{R}^{k} \rightarrow \mathbb{R}^{k \times k}$ are given functions, $B(t)$ is a $k$-dimensional Brownian motion and $\tilde{N}(.,)=.\left(\tilde{N}_{1}(.,),. \ldots, \tilde{N}_{k}(.,).\right)$ are $k$ independent compensated Poisson random measures independent of $B($.$) , while K$ is a given subset of $\mathbb{R}^{p}$. For each $j=1, \ldots, k$ we have $\tilde{N}_{j}(d t, d z)=N_{j}(d t, d z)-\nu_{j}(d z) d t$, where $\nu_{j}$ is the Lévy measure (intensity measure) of the Poisson random measure $N_{j}(.,$.$) .$

We may regard $u(t, z)=\left(u_{0}(t), u_{1}(t, z)\right)$ as our control process, assumed to be càdlàg, $\mathcal{F}_{t^{-}}$ adapted and with values in $K \times K$ for a.a. $t, z, \omega$. Thus $Y(t)=Y^{(u)}(t)$ is a controlled jump diffusion.

Let $f: \mathbb{R}^{k} \times K \rightarrow \mathbb{R}$ and $g: \mathbb{R}^{k} \rightarrow \mathbb{R}$ be given functions. Let $\mathcal{A}$ be a given set of controls contained in the set of $u=\left(u_{0}, u_{1}\right)$ such that (1.3) has a unique strong solution and such that

$$
\mathbb{E}^{y}\left[\int_{0}^{\tau_{\delta}}|f(Y(t), u(t))| d t\right]<\infty
$$

(where $\mathbb{E}^{y}$ denotes expectation when $Y(0)=y$ ) where

$$
\tau_{\mathcal{S}}=\inf \{t>0 ; Y(t) \notin \mathcal{S}\} \quad(\text { the bankruptcy time) }
$$

is the first exit time of a given open solvency set $\mathcal{S} \subset \mathbb{R}^{k}$. We let $\mathcal{T}$ denote the set of all stopping times $\tau \leq \tau_{\mathcal{S}}$. We assume that

$$
\left\{g^{-}(Y(\tau))\right\}_{\tau \in \mathcal{T}} \quad \text { is uniformly integrable. }
$$

For $\tau \in \mathcal{T}$ and $u \in \mathcal{A}$ we define the performance functional $J^{\tau, u}(y)$ by

$$
J^{\tau, u}(y)=\mathbb{E}^{y}\left[\int_{0}^{\tau} f(Y(t), u(t)) d t+g(Y(\tau))\right] .
$$

Where

$$
\tau_{\mathcal{S}}=\inf \{t>0 ; Y(t) \notin \mathcal{S}\} \quad \text { (the bankruptcy time) }
$$

is the first exit time of a given open solvency set $\mathcal{S} \subset \mathbb{R}^{k}$. We regard $\tau$ as the "control" of player number 1 and $u$ as the control of player number 2, and consider the stochastic differential game to find the value function $\Phi$ and an optimal pair $\left(\tau^{*}, u^{*}\right) \in \mathcal{T} \times \mathcal{A}$ such that

$$
\Phi(y)=\inf _{u \in \mathcal{A}}\left(\sup _{\tau \in \mathcal{T}} J^{\tau, u}(y)\right)=J^{\tau^{*}, u^{*}}(y) .
$$


Let $A^{u}$ be the generator given by

$$
\begin{aligned}
A^{u} \varphi(y)= & \sum_{i=1}^{k} b_{i}\left(y, u_{0}(y)\right) \frac{\partial \varphi}{\partial y_{i}}(y) \\
+ & \frac{1}{2} \sum_{i, j=1}^{k}\left(\sigma \sigma^{t}\right)_{i j}\left(y, u_{0}(y)\right) \frac{\partial^{2} \varphi}{\partial y_{i} \partial y_{j}}(y) \\
+ & \sum_{j=1}^{k} \int_{\mathbb{R}}\left\{\varphi \left(y+\gamma^{(j)}\left(y, u_{1}(y, z), z\right)-\varphi(y)\right.\right. \\
& \left.-\nabla \varphi(y) \cdot \gamma^{(j)}\left(y, u_{1}(y, z), z\right)\right\} \nu_{j}(d z) ; \quad \varphi \in \mathcal{C}^{2}\left(\mathbb{R}^{k}\right) .
\end{aligned}
$$

Here $\nabla \varphi=\left(\frac{\partial \varphi}{\partial y_{1}}, \ldots, \frac{\partial \varphi}{\partial y_{k}}\right)$ is the gradient of $\varphi$ and $\gamma^{(j)}$ is column number $j$ of the $k \times k$ matrix $\gamma$. We then have the following verification theorem.

Theorem 1.1. (Verification theorem for stopping-control games)

Suppose there exists a function $\varphi: \overline{\mathcal{S}} \rightarrow \mathbb{R}$ such that

(i) $\varphi \in \mathcal{C}^{1}(\mathcal{S}) \bigcap \mathcal{C}(\bar{S})$

(ii) $\varphi \geq g$ on $\mathcal{S}$

Define

$$
D=\{y \in \mathcal{S} ; \varphi(y)>g(y)\} \quad \text { (the continuation region) }
$$

Suppose, with $Y(t)=Y^{(u)}(t)$,

(iii) $\mathbb{E}^{y}\left[\int_{0}^{\tau_{S}} \chi_{\partial D}(Y(t)) d t\right]=0$ for all $u \in \mathcal{A}$

(iv) $\partial D$ is a Lipschitz surface

(v) $\varphi \in \mathcal{C}^{2}(\mathcal{S} \backslash \partial D)$, with locally bounded derivatives near $\partial D$

(vi) there exists $\hat{u} \in \mathcal{A}$ such that

$$
A^{\hat{u}} \varphi(y)+f(y, \hat{u}(y))=\inf _{u \in \mathcal{A}}\left\{A^{u} \varphi(y)+f(y, u(y))\right\}\left\{\begin{array}{l}
=0, \text { for } y \in D, \\
\leq 0, \text { for } y \in \mathcal{S} \backslash D .
\end{array}\right.
$$

(vii) $\mathbb{E}^{y}\left[|\varphi(Y(\tau))|+\int_{0}^{\tau}\left|A^{u} \varphi(Y(t))\right| d t\right]<\infty$, for all $\tau \in \mathcal{T}$ and all $u \in \mathcal{A}$.

For $u \in \mathcal{A}$ define

$$
\tau_{D}=\tau_{D}^{(u)}=\inf \left\{t>0 ; Y^{(u)}(t) \notin D\right\}
$$

and, in particular,

$$
\hat{\tau}=\tau_{D}^{(\hat{u})}=\inf \left\{t>0 ; Y^{(\hat{u})}(t) \notin D\right\} .
$$

(viii) Suppose that the family $\left\{\varphi(Y(\tau)) ; \tau \in \mathcal{T}, \tau \leq \tau_{D}\right\}$ is uniformly integrable, for each $u \in \mathcal{A}, y \in \mathcal{S}$.

Then $\varphi(y)=\Phi(y)$ and $(\hat{\tau}, \hat{u}) \in \mathcal{T} \times \mathcal{A}$ is an optimal pair, in the sense that

$$
\Phi(y)=\inf _{u}\left(\sup _{\tau} J^{\tau, u}(y)\right)=\sup _{\tau} J^{\tau, \hat{u}}(y)=J^{\hat{\tau}, \hat{u}}(y)=\varphi(y)=\inf _{u} J^{\tau_{D}, u}(y)=\sup _{\tau}\left(\inf _{u} J^{\tau, u}(y)\right) .
$$




\subsection{Maximum principles for jump diffusion processes with infinite horizon}

The second article is entitled "Maximum principles for jump diffusion processes with infinite horizon". It is written in collaboration with Bernt Øksendal and Frank Proske and is published in Automatica.

In this article we consider a controlled jump diffusion, $X(t)=X^{u}(t)$, described by the stochastic differential equation

$$
\begin{aligned}
d X(t) & =b(t, X(t), u(t), \omega) d t+\sigma(t, X(t), u(t), \omega) d B(t) \\
& +\int_{\mathbb{R}_{0}^{n}} \theta(t, X(t), u(t), z, \omega) \tilde{N}(d z, d t) ; 0 \leq t<\infty \\
X(0) & =x \in \mathbb{R}^{n}
\end{aligned}
$$

where $\mathbb{R}_{0}=\mathbb{R} \backslash\{0\}, b:[0, \infty] \times \mathbb{R}^{n} \times U \times \Omega \rightarrow \mathbb{R}^{n}$ is adapted, $\sigma:[0, \infty] \times \mathbb{R}^{n} \times U \times \Omega \rightarrow \mathbb{R}^{n \times n}$ is adapted with respect to $\left\{\mathcal{F}_{t}\right\}_{t \geq 0}$ and $\theta:[0, \infty] \times \mathbb{R}^{n} \times U \times \Omega \rightarrow \mathbb{R}^{n \times n}$ is predictable with respect to $\left\{\mathcal{F}_{t}\right\}_{t \geq 0}$.

Let

$$
\mathcal{E}_{t} \subset \mathcal{F}_{t},
$$

be a given subfiltration, representing the information available to the controller at time $t ; t \geq 0$. The process $u(t)$ is our control, assumed to be $\left\{\mathcal{E}_{t}\right\}_{t \geq 0}$ predictable and with values in a convex set $U \subset \mathbb{R}^{n}$.

Now, consider a control problem for a performance functional given by

$$
J(u)=E\left[\int_{0}^{\infty} f(t, X(t), u(t), \omega) d t\right]
$$

where $X(t)$ is a controlled jump diffusion and $u(t)$ is the control process. We allow for the case where the controller only has access to partial information. Thus, we have an infinite horizon problem with partial information. Namely, the problem to find $\hat{u} \in \mathcal{A}_{\varepsilon}$ such that

$$
J(\hat{u})=\sup _{u \in \mathcal{A}_{\varepsilon}} J(u)
$$

Let us define the Hamiltonian $H:[0, T] \times \mathbb{R}^{n} \times U \times \mathbb{R}^{n} \times \mathbb{R}^{n \times n} \times \mathcal{R} \rightarrow \mathbb{R}$, by

$$
\begin{aligned}
H(t, x, u, p, q, r) & =f(t, x, u, \omega)+b^{T}(t, x, u, \omega) p+\operatorname{tr}\left(\sigma^{T}(t, x, u, \omega) q\right) \\
& +\sum_{i, j=1}^{n} \int_{\mathbb{R}_{0}^{n}} \theta_{i, j}(t, x, u, z, \omega) r_{i, j}(t, z) \nu_{j}(d z) .
\end{aligned}
$$


The adjoint equation in the unknown $\mathcal{F}_{t}$-predictable processes $(p(t), q(t), r(t, z))$ is the following

$$
\begin{aligned}
d p(t) & =-\nabla_{x} H(t, X(t), u(t), p(t), q(t), r(t, \cdot)) d t+q(t) d B(t) \\
& +\int_{\mathbb{R}_{0}^{n}} r(t, z) \tilde{N}(d z, d t), t \geq 0 .
\end{aligned}
$$

The first we do is to prove existence and uniqueness of the solution $(Y(t), Z(t), K(t, \zeta))$ of infinite horizon BSDEs of the form;

$$
\begin{aligned}
d Y(t) & =-g(t, Y(t), Z(t), K(t, \cdot)) d t+Z(t) d B(t) \\
& +\int_{\mathbb{R}_{0}^{n}} K(t, \zeta) \tilde{N}(d \zeta, d t) ; 0 \leq t \leq \tau, \\
\lim _{t \rightarrow \tau} Y(t) & =\xi(\tau) \mathbf{1}_{[0, \infty)}(\tau),
\end{aligned}
$$

where $\tau \leq \infty$ is a given $\mathcal{F}_{t}$-stopping time, possibly infinite.

To state our main result for infinite horizon BSDEs we need to list some requirements:

1. The function $g: \Omega \times \mathbb{R}_{+} \times \mathbb{R}^{k} \times \mathbb{R}^{k \times d} \times \mathcal{R} \rightarrow \mathbb{R}^{k}$ is such that there exist real numbers $\mu, \lambda, K_{1}$ and $K_{2}$, such that $K_{1}, K_{2}>0$ and

$$
\lambda>2 \mu+K_{1}^{2}+K_{2}^{2}
$$

We assume that the function $g$ satisfies the following requirements:

(a) $g(\cdot, y, z, k)$ is progressively measurable for all $y, z, k$, and

$$
\begin{aligned}
& \left|g(t, y, z, k(\cdot))-g\left(t, y, z^{\prime}, k^{\prime}(\cdot)\right)\right| \leq K_{1}\left\|z-z^{\prime}\right\| \\
& +K_{2}\left\|k(\cdot)-k^{\prime}(\cdot)\right\|_{R},
\end{aligned}
$$

where

$$
\|k(\cdot)\|_{R}^{2}=\int_{\mathbb{R}_{0}^{n}} k^{2}(\zeta) \nu(d \zeta)
$$

and $\|z\|=\left[\operatorname{Tr}\left(z z^{*}\right)\right]^{\frac{1}{2}}$.

(b)

$$
\left\langle y-y^{\prime}, g(t, y, z, k)-g\left(t, y^{\prime}, z, k\right)\right\rangle \leq \mu\left|y-y^{\prime}\right|^{2}
$$

for all $y, y^{\prime}, z, k$ P-a.s.

(c)

$$
E \int_{0}^{\tau} e^{\lambda t}|g(t, 0,0,0)|^{2} d t<\infty
$$

(d) Finally we require that

$$
y \mapsto g(t, y, z, k)
$$

is continuous for all $t, z, k$ P-a.s. 
2. We have a final condition on $\xi$, which is $\mathcal{F}_{\tau}$-measurable such that $E\left(e^{\lambda \tau}|\xi|^{2}\right)<\infty$ and

$$
E \int_{0}^{\tau} e^{\lambda t}\left|g\left(t, \xi_{t}, \eta_{t}, \psi_{t}\right)\right|^{2} d t<\infty
$$

where $\xi_{t}=E\left(\xi \mid \mathcal{F}_{t}\right)$ and $\eta, \psi$ are $\left\{\mathcal{F}_{t}\right\}_{t \geq 0}$-adapted and $\left\{\mathcal{F}_{t}\right\}_{t \geq 0}$-predictable, respectively, s.t.

$$
\xi=E \xi+\int_{0}^{t} \eta(s) d B_{s}+\int_{0}^{t} \int_{\mathbb{R}_{0}^{n}} \psi(s, \zeta) \tilde{N}(d \zeta, d s) .
$$

A solution of the BSDE (1.7)-(1.8), is a triple $\left(Y_{t}, Z_{t}, K_{t}\right)$ of progressively measurable processes with values in $\mathbb{R}^{k} \times \mathbb{R}^{k \times d} \times \mathcal{R}$ s.t. $Z_{t}, K_{t}=0$ when $t>\tau$,

1. $E\left[\sup _{t \geq 0} e^{\lambda t}\left|Y_{t}\right|^{2}+\int_{0}^{\tau} e^{\lambda s}\left|Z_{s}\right|^{2} d s+\int_{0}^{\tau} \int_{\mathbb{R}_{0}^{n}} e^{\lambda s} K^{2}(s, \zeta) \nu(d \zeta) d s\right]<\infty$,

2. $Y_{t}=Y_{T \wedge \tau}+\int_{t \wedge \tau}^{T \wedge \tau} g_{s} d s-\int_{t \wedge \tau}^{T \wedge \tau} Z_{s} d B_{s}-\int_{t \wedge \tau}^{T \wedge \tau} \int_{\mathbb{R}_{0}^{n}} K(s, \zeta) \tilde{N}(d \zeta, d s)$ for all deterministic $T<\infty$ and

3. $Y_{t}=\xi$ on the set $\{t \geq \tau\}$.

Remark 1.1 (Infinite Horizon). This incorporates the case where $\tau(\omega)=\infty$ on some set $A$ with $P(A)>0$, possibly $P(A)=1$.

Theorem 1.2 (Existence and uniqueness). Under the above conditions there exists a unique solution $\left(Y_{t}, Z_{t}, K_{t}\right)$ of the BSDE (1.7)-(1.8), which satisfies the condition;

$$
\begin{aligned}
& E\left[\sup _{0 \leq t \leq \tau} e^{\lambda t}\left|Y_{t}\right|^{2}+\int_{0}^{\tau} e^{\lambda s}\left(\left|Y_{s}\right|^{2}+\left\|Z_{s}\right\|^{2}\right) d s+\int_{0}^{\tau} e^{\lambda s} \int_{\mathbb{R}_{0}^{n}} K^{2}(s, \zeta) \nu(d \zeta) d s\right] \\
& \leq c E\left[e^{\lambda \tau}|\xi|^{2}+\int_{0}^{\tau} e^{\lambda s}|g(s, 0,0,0)|^{2} d s\right]
\end{aligned}
$$

for some positive number $c$.

We are now able to present the main result of this paper, namely the following maximum principle:

Theorem 1.3 (Sufficient Infinite Horizon Maximum Principle). Let $\hat{u} \in \mathcal{A}_{\varepsilon}$ and let $(\hat{p}(t), \hat{q}(t), \hat{r}(t, z))$ be an associated solution to the equation (1.6). Assume that for all $u \in \mathcal{A}_{\mathcal{E}}$ the following terminal condition holds:

$$
0 \leq E\left[\varlimsup_{t \rightarrow \infty}\left[\hat{p}(t)^{T}(X(t)-\hat{X}(t))\right]\right]<\infty
$$

Moreover, assume that $H(t, x, u, \hat{p}(t), \hat{q}(t), \hat{r}(t, \cdot))$ is concave in $x$ and $u$ and

$$
\begin{array}{r}
E\left[H(t, \hat{X}(t), \hat{u}(t), \hat{p}(t), \hat{q}(t), \hat{r}(t, \cdot)) \mid \varepsilon_{t}\right] \\
=\max _{u \in U} E\left[H(t, \hat{X}(t), u, \hat{p}(t), \hat{q}(t), \hat{r}(t, \cdot)) \mid \varepsilon_{t}\right] .
\end{array}
$$


In addition we assume that for all $T<\infty$,

$$
\begin{aligned}
& E\left[\int _ { 0 } ^ { T } ( \hat { X } ( t ) - X ^ { u } ( t ) ) ^ { T } \left[\hat{q} \hat{q}^{T}\right.\right. \\
& \left.\left.+\int_{\mathbb{R}_{0}^{n}} \hat{r} \hat{r}^{T}(t, z) \nu(d z)\right]\left(\hat{X}(t)-X^{u}(t)\right) d t\right]<\infty, \\
& E\left[\int _ { 0 } ^ { T } \hat { p } ( t ) ^ { T } \left[\sigma \sigma^{T}(t, X(t), u(t))\right.\right. \\
& \left.\left.+\int_{\mathbb{R}_{0}^{n}} \theta \theta^{T}(t, X(t), u(t), z) \nu(d z)\right] p(t) d t\right]<\infty, \\
& E\left[\left|\nabla_{u} H(t, \hat{X}(t), \hat{u}(t), \hat{p}(t), \hat{q}(t), \hat{r}(t, \cdot))\right|^{2}\right]<\infty,
\end{aligned}
$$

and that

$$
E\left[\int_{0}^{\infty}|H(s, X(s), u(s), \hat{p}(s), \hat{q}(s), \hat{r}(s, \cdot))|\right]<\infty
$$

for all $u$.

Then we have that $\hat{u}(t)$ is optimal.

We continue the article by giving some examples before we give a necessary maximum principle. The necessary maximum principle will delayed to the full article as it is very technical.

\subsection{A Maximum Principle for Infinite Horizon Delay Equa- tions}

The third article is entitled "A Maximum Principle for Infinite Horizon Delay Equations". It is written in collaboration with Nacira Agram, Bernt Øksendal and Frank Proske. The article is to be published in SIAM.

In this article we consider a system that is governed by the stochastic differential delay equation (SDDE in short):

$$
\left\{\begin{aligned}
d X(t) & =b(t, X(t), Y(t), A(t), u(t)) d t \\
& +\sigma(t, X(t), Y(t), A(t), u(t)) d B(t) \\
& +\int_{\mathbb{R}_{0}} \theta(t, X(t), Y(t), A(t), u(t), z) \tilde{N}(d t, d z) ; t \in[0, \infty), \\
X(t) & =X_{0}(t) ; t \in[-\delta, 0] \\
Y(t) & =X(t-\delta) ; t \in[0, \infty), \\
A(t) & =\int_{t-\delta}^{t} e^{-\lambda(t-r)} X(r) d r ; t \in[0, \infty),
\end{aligned}\right.
$$


where $X_{0}(t)$ is a given continuous (deterministic) function, and

$$
\begin{aligned}
& \delta>0, \lambda>0 \text { are given constants. } \\
& b:[0, \infty) \times \mathbb{R} \times \mathbb{R} \times \mathbb{R} \times \mathcal{U} \times \Omega \rightarrow \mathbb{R} \\
& \sigma:[0, \infty) \times \mathbb{R} \times \mathbb{R} \times \mathbb{R} \times \mathcal{U} \times \Omega \rightarrow \mathbb{R} \\
& \theta:[0, \infty) \times \mathbb{R} \times \mathbb{R} \times \mathbb{R} \times \mathcal{U} \times \mathbb{R}_{0} \times \Omega \rightarrow \mathbb{R}
\end{aligned}
$$

are given continuous functions such that for all $t, b(t, x, y, a, u), \sigma(t, x, y, a, u)$ and $\theta(t, x, y, a, u, z)$ are $\mathcal{F}_{t}$-measurable for all $x \in \mathbb{R}, y \in \mathbb{R}, a \in \mathbb{R}, u \in \mathcal{U}$ and $z \in \mathbb{R}_{0}:=\mathbb{R} \backslash\{0\}$. We assume that $b, \sigma, \theta$ are $C^{1}$ (i.e. continuously differentiable/ Frèchet differentiable) with respect to $x, y, a, u$ and $z$, for all $t$ and a.a $\omega$. Let $\varepsilon_{t} \subset \mathcal{F}_{t}$ be a given subfiltration, representing the information available to the controller at time $t$. Let $\mathcal{U}$ be a non-empty subset of $\mathbb{R}$. We let $\mathcal{A}_{\varepsilon}$ denote a given family of admissible $\mathcal{E}_{t}$-adapted control processes. An element of $\mathcal{A}_{\mathcal{\varepsilon}}$ is called an admissible control. The corresponding performance functional is given as

$$
J(u)=E\left[\int_{0}^{\infty} f(t, X(t), Y(t), A(t), u(t)) d t\right] ; u \in \mathcal{A}_{\mathcal{E}},
$$

where $u(t)$ is the control process.

The value function $\Phi$ is defined as

$$
\Phi\left(X_{0}\right)=\sup _{u \in \mathcal{A}_{\varepsilon}} J(u)
$$

An admissible control $u^{*}$ is called an optimal control for (1.9) if it attains the maximum of $J(u)$ over $\mathcal{A}_{\mathcal{E}}$. (1.9) is called the state equation, the solution $X^{*}(t)$ corresponding to $u^{*}$ is called an optimal trajectory.

The Hamiltonian is defined by

$$
\begin{aligned}
& H(t, x, y, a, u, p, q, r(\cdot))=f(t, x, y, a, u)+b(t, x, y, a, u) p+\sigma(t, x, y, a, u) q \\
&+\int_{\mathbb{R}_{0}} \theta(t, x, y, a, u, z) r(z) \nu(d z)
\end{aligned}
$$

where

$$
H:[0, \infty) \times \mathbb{R} \times \mathbb{R} \times \mathbb{R} \times \mathcal{U} \times \mathbb{R} \times \mathbb{R} \times \Re \times \Omega \rightarrow \mathbb{R}
$$

and $\Re$ is the set of functions $r: \mathbb{R}_{0} \rightarrow \mathbb{R}$ such that the integral term in (1.12) converges and $\mathcal{U}$ is the set of possible control values.

We suppose that $b, \sigma$ and $\theta$ are $C^{1}$ functions with respect to $(x, y, a, u)$ and that

$$
\begin{gathered}
E\left[\int _ { 0 } ^ { \infty } \left\{\left|\frac{\partial b}{\partial x_{i}}(t, X(t), Y(t), A(t), u(t))\right|^{2}+\left|\frac{\partial \sigma}{\partial x_{i}}(t, X(t), Y(t), A(t), u(t))\right|^{2}\right.\right. \\
\left.\left.+\int_{\mathbb{R}_{0}}\left|\frac{\partial \theta}{\partial x_{i}}(t, X(t), Y(t), A(t), u(t))\right|^{2} \nu(d z)\right\} d t\right]<\infty
\end{gathered}
$$

for $x_{i}=x, y, a$ and $u$. 

tion

The adjoint processes $(p(t), q(t), r(t, \cdot)), t \in[0, \infty), z \in \mathbb{R}_{0}$ are assumed to satisfy the equa-

$$
d p(t)=E\left[\mu(t) \mid \mathcal{F}_{t}\right] d t+q(t) d B(t)+\int_{\mathbb{R}_{0}} r(t, z) \tilde{N}(d t, d z) ; t \in[0, \infty)
$$

where

$$
\begin{aligned}
& \mu(t)=-\frac{\partial H}{\partial x}(t, X(t), Y(t), A(t), u(t), p(t), q(t), r(t, \cdot)) \\
& -\frac{\partial H}{\partial y}(t+\delta, X(t+\delta), Y(t+\delta), A(t+\delta), u(t+\delta), p(t+\delta), q(t+\delta), r(t+\delta, \cdot)) \\
& -e^{\lambda t}\left(\int_{t}^{t+\delta} \frac{\partial H}{\partial a}(s, X(s), Y(s), A(s), u(s), p(s), q(s), r(s, \cdot)) e^{-\lambda s} d s\right) .
\end{aligned}
$$

We then show the following theorem;

Theorem 1.4. Let $\hat{u} \in \mathcal{A}_{\varepsilon}$ with corresponding state processes $\hat{X}(t), \hat{Y}(t)$ and $\hat{A}(t)$ and adjoint processes $\hat{p}(t), \hat{q}(t)$ and $\hat{r}(t, \cdot)$ assumed to satisfy the advanced backward stochastic differential equation (ABSDE in short) (1.14)- (1.15). Suppose that the following assertions hold:

(i)

$$
\varlimsup_{T \rightarrow \infty} E[\hat{p}(T)(X(T)-\hat{X}(T))] \geq 0,
$$

for all $u \in \mathcal{A}_{\varepsilon}$ with corresponding solution $X(t)$.

(ii) The function

$$
(x, y, a, u) \rightarrow H(t, x, y, a, u, \hat{p}(t), \hat{q}(t), \hat{r}(t, \cdot)),
$$

is concave for each $t \in[0, \infty)$ a.s.

(iii)

$$
E\left[\int_{0}^{T}\left\{\hat{q}^{2}(t)(\sigma(t)-\hat{\sigma}(t))^{2}+\int_{\mathbb{R}} \hat{r}^{2}(t, z)(\theta(t, z)-\hat{\theta}(t, z))^{2} \nu(d z)\right\} d t\right]<\infty,
$$

for all $T<\infty$.

(iiii)

$$
\begin{aligned}
& \max _{v \in \mathcal{U}} E\left[H(t, \hat{X}(t), \hat{X}(t-\delta), \hat{A}(t), v, \hat{p}(t), \hat{q}(t), \hat{r}(t, \cdot)) \mid \mathcal{E}_{t}\right] \\
& =E\left[H(t, \hat{X}(t), \hat{X}(t-\delta), \hat{A}(t), \hat{u}(t), \hat{p}(t), \hat{q}(t), \hat{r}(t, \cdot)) \mid \varepsilon_{t}\right],
\end{aligned}
$$

for all $t \in[0, \infty)$ a.s.

Then $\hat{u}$ is an optimal control for the problem (1.11).

We also show a jump version of an Itô formula for a delayed system. 
Lemma 1.5 (The Itô formula for delayed system). Consider a function

$$
G(t)=F(t, X(t), A(t))
$$

where $F$ is a function in $C^{1,2,1}\left(\mathbb{R}^{3}\right)$. Note that

$$
A(t)=\int_{-\delta}^{0} e^{\lambda s} X(t+s) d s .
$$

Then

$$
\begin{aligned}
d G(t)= & (L F)(t, X(t), Y(t), A(t), u(t)) d t \\
+ & \sigma(t, X(t), Y(t), A(t), u(t)) \frac{\partial F}{\partial x}(t, X(t), A(t)) d B(t) \\
+ & \int_{\mathbb{R}_{0}}\left\{F\left(t, X\left(t^{-}\right)+\theta(t, X(t), Y(t), A(t), u, z), A\left(t^{-}\right)\right)\right. \\
& -F\left(t, X\left(t^{-}\right), A\left(t^{-}\right)\right) \\
& \left.-\frac{\partial F}{\partial x}\left(t, X\left(t^{-}\right), A\left(t^{-}\right)\right) \theta(t, X(t), Y(t), A(t), u, z)\right\} \nu(d z) d t \\
+ & \int_{\mathbb{R}_{0}}\left\{F\left(t, X\left(t^{-}\right)+\theta(t, X(t), Y(t), A(t), u, z), A\left(t^{-}\right)\right)\right. \\
& \left.-F\left(t, X\left(t^{-}\right), A\left(t^{-}\right)\right)\right\} \tilde{N}(d t, d z) \\
+ & {\left[X(t)-\lambda A(t)-e^{-\lambda \delta} Y(t)\right] \frac{\partial F}{\partial a}(t, X(t), A(t)) d t, }
\end{aligned}
$$

where

$$
L F=L F(t, x, y, a, u)=\frac{\partial F}{\partial t}+b(t, x, y, a, u) \frac{\partial F}{\partial x}+\frac{1}{2} \sigma^{2}(t, x, y, a, u) \frac{\partial^{2} F}{\partial x^{2}} .
$$

This formula allows us to show a second maximum principle.

First, define the Hamiltonian, $H^{\prime}: \mathbb{R}_{+} \times \mathbb{R} \times \mathbb{R} \times \mathbb{R} \times \mathcal{U} \times \mathbb{R}^{3} \times \mathbb{R}^{3} \times \Re \rightarrow \mathbb{R}$ as

$$
\begin{aligned}
& H^{\prime}(t, x, y, a, u, p, q, r(\cdot)) \\
& =f(t, x, y, a, u)+b(t, x, y, a, u) p_{1}+\left(x-\lambda a-e^{-\lambda \delta} y\right) p_{3} \\
& +\sigma(t, x, y, a, u) q_{1}+\int_{\mathbb{R}_{0}} \theta(t, x, y, a, u, z) r(z) \nu(d z),
\end{aligned}
$$

where $p=\left(p_{1}, p_{2}, p_{3}\right)^{T} \in \mathbb{R}^{3}$ and $q=\left(q_{1}, q_{2}, q_{3}\right) \in \mathbb{R}^{3}$. For each $u \in \mathcal{A}_{\varepsilon}$ the associated adjoint equations are the following backward stochastic differential equations in the unknown 
$\mathcal{F}_{t}$-adapted processes $(p(t), q(t), r(t, \cdot))$ given by

$$
\begin{aligned}
d p_{1}(t) & =-\frac{\partial H^{\prime}}{\partial x}(t, X(t), Y(t), A(t), u(t), p(t), q(t), r(t, \cdot)) d t+q_{1}(t) d B(t) \\
& +\int_{\mathbb{R}_{0}} r(t, z) \tilde{N}(d t, d z) \\
d p_{2}(t) & =-\frac{\partial H^{\prime}}{\partial y}(t, X(t), Y(t), A(t), u(t), p(t), q(t), r(t, \cdot)) d t \\
d p_{3}(t) & =-\frac{\partial H^{\prime}}{\partial a}(t, X(t), Y(t), A(t), u(t), p(t), q(t), r(t, \cdot)) d t+q_{3}(t) d B(t),
\end{aligned}
$$

Theorem 1.6 (A second infinite horizon maximum principle for delay equations). Suppose $\hat{u} \in \mathcal{A}_{\varepsilon}$ and let $(\hat{X}(t), \hat{Y}(t), \hat{A}(t))$ and $(\hat{p}(t), \hat{q}(t), \hat{r}(t, \cdot))$ be corresponding solutions of (1.21)(1.23), respectively. Suppose that

$$
\left.(x, y, a, u) \mapsto H^{\prime}(t, x, y, a, u, \hat{p}(t), \hat{q}(t), \hat{r}(t, \cdot))\right)
$$

is concave for all $t \geq 0$ a.s. and

$$
\begin{aligned}
& E\left[H^{\prime}(t, \hat{X}(t), \hat{Y}(t), \hat{A}(t), \hat{u}(t), \hat{p}(t), \hat{q}(t), \hat{r}(t, \cdot)) \mid \mathcal{E}_{t}\right] \\
& =\max _{u \in \mathcal{U}} E\left[H^{\prime}(t, \hat{X}(t), \hat{Y}(t), \hat{A}(t), u, \hat{p}(t), \hat{q}(t), \hat{r}(t, \cdot)) \mid \mathcal{E}_{t}\right] .
\end{aligned}
$$

Further, assume that

$$
\varlimsup_{T \rightarrow \infty} E\left[\hat{p}_{1}(T)(X(T)-\hat{X}(T))+\hat{p}_{3}(T)(A(T)-\hat{A}(T))\right] \geq 0
$$

In addition assume that

$$
\hat{p}_{2}(t)=0,
$$

for all $t$. Then $\hat{u}$ is an optimal control for the control problem (1.11).

We then show some examples before we prove a necessary maximum principle.

\subsection{Backward Stochastic fractional differential equations and a maximum principle with infinite horizon driven by a fractional Brownian motion}

The fourth article is entitled "Backward Stochastic fractional differential equations and a maximum principle with infinite horizon driven by a fractional Brownian motion".

In this article we consider a control problem with respect to a given performance functional:

$$
J(u)=E\left[\int_{0}^{\infty} f(t, X(t), u(t), \omega) d t\right],
$$


where $X(t)$ is a controlled fractional diffusion given by

$$
\begin{aligned}
d X(t) & =b(t, X(t), u(t), \omega) d t+\sigma(t, X(t), u(t), \omega) d B^{H}(t) \\
X(0) & =x \in \mathbb{R}^{n},
\end{aligned}
$$

where $B_{t}^{H}$ is a fractional Brownian motion with Hurst parameter $H$ and $u(t)$ is the control process.

We start by taking a look at fractional backward stochastic differential equations. Let $b$ : $[0, \infty) \times \mathbb{R} \times \mathbb{R} \rightarrow \mathbb{R}$. Consider the problem of finding a $\mathcal{F}^{H}$-adapted processes $(p(t), q(t))$ such that

$$
\begin{cases}d p(t) & =b(t, p(t), q(t)) d t+q(t) d B^{H}(t) \\ \lim _{t \rightarrow \infty} p(t) & =0\end{cases}
$$

This is a infinite horizon fractional backward stochastic differential equation (ihfBSDE). We prove a result about existence and uniqueness of the solution $(Y(t), Z(t))$ of infinite horizon BSDEs of the form;

$$
\begin{aligned}
d Y(t) & =-g(t, Y(t), Z(t)) d t+Z(t) d B^{H}(t) ; 0 \leq t \leq \tau, \\
\lim _{t \rightarrow \tau} Y(t) & =\xi(\tau) \mathbf{1}_{[0, \infty)}(\tau),
\end{aligned}
$$

where $\tau \leq \infty$ is a given $\mathcal{F}_{t}$-stopping time, possibly infinite. Our result is the fractional version of Haadem et al. [2013], and the infinite horizon version of Baghery et al. [2008]. See also Pardoux [1999], Peng and Shi [2000], Yin [2008], Li and Peng [2009], Li and Tang [1994], Royer [2006], Barles et al. [2009] and Situ [2002], for the classical Brownian motion case.

Let $\eta_{t}=\eta_{0}+b_{t}+\int_{0}^{t} \sigma_{s} d B_{s}^{H}$, where $\eta_{0}$ is a given constant, $b_{t}$ is a deterministic differentiable function of $t$, and $\sigma_{s}$ is a deterministic continuous function such that $\sigma_{t}$ exists for all $t$ and $\frac{d}{d t}\|\sigma\|_{t}$ exists and it is strictly positive. Let $\xi=h\left(\eta_{\tau}\right)$ and a continuous $h$. Let

$$
p_{t}(x)=\frac{1}{\sqrt{2 \pi t}} e^{-\frac{x^{2}}{2 t}}
$$

Let the semigroup $\left\{P_{t}\right\}_{t \geq 0}$ be given by

$$
P_{t} f(x)=\int_{\mathbf{R}} p_{t}(x-y) f(y) d y .
$$

If $f$ is continuous we have that

$$
\frac{\partial}{\partial t} P_{t} f(x)=\frac{1}{2} \frac{\partial^{2}}{\partial x^{2}} P_{t} f(x)
$$

Using Itô's Lemma, we get

$$
f\left(\eta_{t}\right)=P_{\|\sigma\|_{t}^{2}} f\left(\eta_{0}\right)+\int_{0}^{T} \frac{\partial}{\partial t} P_{\|\sigma\|_{t}^{2}-\|\sigma\|_{s}^{2}} f\left(\eta_{s}\right) \sigma(s) d B_{s}^{H} .
$$


Therefore

$$
\hat{E}\left[f\left(\eta_{t}\right) \mid \mathcal{F}_{t}\right]=P_{\|\sigma\|_{t}^{2}} f\left(\eta_{0}\right)+\int_{0}^{t} \frac{\partial}{\partial t} P_{\|\sigma\|_{t}^{2}-\|\sigma\|_{s}^{2}} f\left(\eta_{s}\right) \sigma(s) d B_{s}^{H} .
$$

Define

$$
\mathcal{V}_{\infty}=\left\{Y(\cdot)=\phi(\cdot, \eta(\cdot)) ; \phi(\cdot, \cdot) \in C^{1,2}\right\}
$$

where $C_{t}^{1,2}$ is the set of functions that are continuously differentiable with respect to $t$ and twice continuously differentiable with respect to $x$. Let $\tilde{\mathcal{V}}_{\infty}$ be the completion of $\mathcal{V}_{\infty}$ under the norm

$$
\|Y\|_{\lambda}^{2}:=\int_{0}^{\infty} e^{\lambda t} E\left[\left|Y_{t}\right|^{2}\right] d t=\int_{0}^{\infty} e^{\lambda t} E\left[\left|\phi\left(t, \eta_{t}\right)\right|^{2}\right] d t .
$$

We require the following:

1. For each $\tau \geq T>0$

$$
\inf _{0 \leq s \leq T} \frac{\hat{\sigma}_{s}}{\sigma_{s}} \geq c_{0},
$$

for some positive constant $c_{0}$, where

$$
\hat{\sigma}_{s}=\int_{0}^{s} \phi(s, r) \sigma_{r} d r
$$

2. We have real numbers $\mu, \lambda$ and $K$, such that $K>0$ and

$$
\lambda>2 \mu+2 K^{2} .
$$

We also assume that the function $g: \Omega \times \mathbb{R}_{+} \times \mathbb{R} \times \mathbb{R} \rightarrow \mathbb{R}$ satisfies the following requirements:

(a) $g(\cdot, y, z)$ is progressively measurable for all $y \in \mathbb{R}, z \in \mathbb{R}$, and

$$
\left|g(t, y, z)-g\left(t, y, z^{\prime}\right)\right| \leq K\left|z-z^{\prime}\right|
$$

(b)

$$
\left\langle y-y^{\prime}, g(t, y, z, k)-g\left(t, y^{\prime}, z, k\right)\right\rangle \leq \mu\left|y-y^{\prime}\right|^{2}
$$

for all $y, y^{\prime}, z$ a.s.

(c)

$$
E \int_{0}^{\tau} e^{\lambda t}|g(t, 0,0)|^{2} d t<\infty
$$

(d) finally we require that

$$
y \mapsto g(t, y, z),
$$

is continuous for all $t, z$ a.s. 
3. Let $\eta_{t}=\eta_{0}+b_{t}+\int_{0}^{t} \sigma_{s} d B_{s}^{H}$ as above. Assume the final condition $\xi$ is given by $\xi=\eta_{\tau}$, and $\xi_{t}=\hat{E}\left[\xi \mid \mathcal{F}_{t}\right]$ such that $E\left[e^{\lambda \tau}|\xi|^{2}\right]<\infty$ and

$$
E\left[\int_{0}^{\tau} e^{\lambda t}\left|g\left(t, \xi_{t}, \sigma_{t}\right)\right|^{2} d t\right]<\infty
$$

A solution of the BSDE (1.24)-(1.25), is a dual $\left(Y_{t}, Z_{t}\right)$ of progressively measurable processes with values in $\mathbb{R} \times \mathbb{R}$ s.t. $Z_{t}=0$ when $t>\tau$,

1. $E\left[\int_{0}^{\tau} e^{\lambda s} D_{t}^{\phi} Z_{s} d s\right]<\infty$,

2. $Y_{t}=Y_{T \wedge \tau}+\int_{t \wedge \tau}^{T \wedge \tau} g_{s} d s-\int_{t \wedge \tau}^{T \wedge \tau} Z_{s} d B_{s}^{H}$ for all deterministic $T<\infty$ and

3. $Y_{t}=\xi$ on the set $\{t \geq \tau\}$.

Remark 1.7 (Infinite Horizon). This incorporates the case where $\tau(\omega)=\infty$ on some set $A$ with $P(A)>0$, possibly $P(A)=1$.

Theorem 1.8 (Existence and uniqueness). Under the above conditions there exists a unique solution $\left(Y_{t}, Z_{t}\right)$ of the BSDE (1.24)-(1.25), which satisfies the condition

$$
\begin{aligned}
& E\left[\int_{0}^{\tau} e^{\lambda s}\left(\left|Y_{s}\right|^{2}+\left|Z_{s}\right|^{2}\right) d s\right] \\
& \leq c E\left[e^{\lambda \tau}|\xi|^{2}+\int_{0}^{\tau} e^{\lambda s}|g(s, 0,0)|^{2} d s\right]
\end{aligned}
$$

for some positive number $c$.

Next we give a constructive proof of existence of the linear infinite horizon backward stochastic differential equation (LIHBSDE)

$$
\begin{aligned}
d p(t) & =[\alpha(t)+b(t) p(t)+c(t) q(t)] d t+q(t) d \hat{B}^{H}(t), \\
\lim _{t \rightarrow \infty} p(t) & =0,
\end{aligned}
$$

where $b(t)$ and $c(t)$ are given continuous deterministic functions and $\alpha(t)=\alpha(t, \omega)$ is a given $\mathcal{F}_{t}^{H}$-adapted process such that $\int_{0}^{\infty}|\alpha(t, \omega)| d t<\infty$ almost surely.

Finally we are able to show our main theorem. Assume we have an m-dimensional fractional Brownian motion, $B^{H}(t)$, with Hurst parameter $H=\left(H_{1}, H_{2}, \ldots, H_{m}\right)$. Let $X(t)=X^{u}(t)$ be a controlled fractional diffusion, described by the stochastic differential equation;

$$
\begin{aligned}
d X(t) & =b(t, X(t), u(t), \omega) d t+\sigma(t, X(t), u(t), \omega) d B^{H}(t) \\
X(0) & =x \in \mathbb{R}^{n} .
\end{aligned}
$$

Let

$$
\mathcal{E}_{t} \subset \mathcal{F}_{t},
$$

be a given subfiltration, representing the information available to the controller at time $t ; t \geq 0$. The process $u(t)$ is our control, assumed to be $\left\{\mathcal{E}_{t}\right\}_{t \geq 0}$ adapted and with values in a set $U \subset \mathbb{R}^{n}$. 
Let $\mathcal{A}$ be our family of $\mathcal{E}_{t}$-adapted controls. Let $f:[0, \infty] \times \mathbb{R}^{n} \times U \times \Omega \rightarrow \mathbb{R}^{n}$ be adapted and assume that

$$
E\left[\int_{0}^{\infty}|f(t, X(t), u(t), \omega)| d t\right]<\infty \text { for all } u \in \mathcal{A}
$$

Then we define

$$
J(u)=E\left[\int_{0}^{\infty} f(t, X(t), u(t), \omega) d t\right]
$$

to be our performance functional.

Definition 1.9 (Admissible pair). Let $\mathcal{A}$ denote the $\mathcal{F}_{t}^{H}$-adapted processes $u:[0, \infty) \times \Omega \rightarrow U$ such that $X^{u}(t)$ exists and doesn't explode in $[0, \infty)$ and such that it satisfies the stochastic differential equation (5.17). If $u \in \mathcal{A}$ and $X^{u}(t)$ is the corresponding state process, we call $\left(u, X^{u}(t)\right)$ an admissible pair.

We study the problem to find $\hat{u} \in \mathcal{A}$ such that

$$
J(\hat{u})=\sup _{u \in \mathcal{A}} J(u)
$$

If such $\hat{u} \in \mathcal{A}$ exists the $\hat{u}$ is called an optimal control and $\left(\hat{u}, X^{\hat{u}}(t)\right)$ is called an optimal pair. Let $C\left([0, \infty), \mathbb{R}^{n \times m}\right)$ be the set of continuous functions from $[0, \infty)$ into $\mathbb{R}^{n \times m}$. We now define the Hamiltonian $H:[0, \infty) \times \mathbb{R}^{n} \times U \times \mathbb{R}^{n} \times C\left([0, \infty), \mathbb{R}^{n \times m}\right) \rightarrow \mathbb{R}$, by

$$
\begin{aligned}
H(t, x, u, p, q, \omega) & =f(t, x, u, \omega)+b^{T}(t, x, u, \omega) p \\
& +\sum_{i=1}^{n} \sum_{j, k=1}^{m} \sigma_{i, k}(t, x, u) \int_{0}^{\infty} q_{i, k}(s) \phi_{H_{k}}(s, t) d s,
\end{aligned}
$$

where $\phi_{H_{k}}(s, t)$ is defined as above. For notational convenience we will in the rest of the paper suppress any $\omega$ in the notation. The adjoint equation that arise in the maximum principle is the unknown $\mathcal{F}_{t}$-predictable processes $(p(t), q(t))$ that satisfies the following stochastic differential equation;

$$
d \hat{p}(t)=-\nabla_{x} H(t, \hat{X}(t), \hat{u}(t), \hat{p}(t), \hat{q}(t)) d t+q(t) d B^{H}(t) .
$$

Theorem 1.10 (Partial Information, Infinite Horizon Fractional Maximum Principle). Let $\hat{u} \in \mathcal{A}$ and let $(\hat{p}(t), \hat{q}(t))$ be an associated solution to the equation (1.34). Define $I_{4}$ as

$$
I_{4}:=E\left[\sum_{i}^{n} \sum_{j, k}^{m} \int_{0}^{\infty} \int_{0}^{\infty} D_{j, s}^{\phi_{j}}\left\{\sigma_{i, k}(t, X(t), u(t))-\sigma_{i, k}(t, \hat{X}(t), \hat{u}(t))\right\} D_{k, t}^{\phi_{k}} \hat{q}_{i, j}(s) d t d s\right] .
$$

Assume that for all $u \in \mathcal{A}$ the following terminal condition holds:

$$
0 \leq E\left[\varlimsup_{t \rightarrow \infty}\left[\hat{p}(t)^{T}(X(t)-\hat{X}(t))\right]\right],
$$


and

$$
I_{4} \leq 0
$$

Moreover assume that $H(t, x, u, \hat{p}(t), \hat{q}(t))$ is concave in $x$ and $u$ and

$$
\begin{array}{r}
E\left[H(t, \hat{X}(t), \hat{u}(t), \hat{p}(t), \hat{q}(t)) \mid \varepsilon_{t}\right] \\
=\max _{u \in U} E\left[H(t, \hat{X}(t), u, \hat{p}(t), \hat{q}(t)) \mid \varepsilon_{t}\right] .
\end{array}
$$

and

$$
E\left[\left|\nabla_{u} H(t, \hat{X}(t), \hat{u}(t), \hat{p}(t), \hat{q}(t))\right|^{2}\right]<\infty
$$

Then we have that $\hat{u}(t)$ is optimal.

\subsection{On the Construction and Malliavin Differentiability of Lévy Noise Driven SDEs with Singular Coefficients}

The fifth article is entitled "On the Construction and Malliavin Differentiability of Lévy Noise Driven SDEs with Singular Coefficients". It is written in collaboration with Frank Proske and is to be published in Journal of Functional Analysis.

In this article we consider the stochastic differential equation (SDE)

$$
X_{t}=x+\int_{0}^{t} b\left(s, X_{s}\right) d s+L_{t}, 0 \leq t \leq T, x \in \mathbb{R}^{d},
$$

where $b:[0, T] \times \mathbb{R}^{d} \longrightarrow \mathbb{R}^{d}$ is a Borel-measurable function and $L_{t}, 0 \leq t \leq T$ is a $d$-dimensional Lévy process. We will study strong solutions to (1.35) for certain Lévy processes, when $b$ is singular in the sense that $b$ is bounded and $\alpha$-Hölder continuous. To achieve this we first show a compactness criterion for subsets of $L^{2}(\mu)$.

Theorem 1.11 (Compactness in $\left.L^{2}(\mu)\right)$. Let $C$ be a self adjoint compact operator on $(H \otimes$ $\left.L^{2}(\nu)\right)$ with dense image, where $H:=L^{2}([0,1])$. Then for any $c>0$ the set

$$
\mathcal{G}=\left\{G \in \mathbb{D}^{1,2}:\|G\|_{L^{2}(\Omega)}+\left\|C^{-1} D G\right\|_{L^{2}\left(\Omega ; H \otimes L^{2}(\nu)\right)} \leq c\right\}
$$

is relatively compact in $L^{2}(\mu)$.

Next, we prove some regularity results for Kolmogorov type equations. We show the following result:

Theorem 1.12. Let $L_{t}, 0 \leq t \leq T$ be a d-dimensional truncated $\alpha$-stable process for $\alpha \in(1,2)$ and $d \geq 2$. Suppose that $\phi \in C\left([0, T], C_{b}^{\beta}\left(\mathbb{R}^{d}\right)\right)$ for $\beta \in(0,1)$ satisfies that $\alpha+\beta>2$. Then there exists a $u \in C\left([0, T], C_{b}^{1, \beta}\left(\mathbb{R}^{d}\right)\right) \cap C\left([0, T], C_{b}^{2}\left(\mathbb{R}^{d}\right)\right) \cap C^{1}\left([0, T], C_{b}\left(\mathbb{R}^{d}\right)\right)$ such that

$$
\frac{\partial u}{\partial t}=\mathcal{L} u+\phi
$$


with $\mathcal{L}$ defined as in (6.9) and such that

$$
\|D u\|_{C_{b}^{\beta}} \leq C(T)\|\phi\|_{C_{b}^{\beta}}
$$

where

$$
C(T) \longrightarrow 0 \text { for } T \searrow 0
$$

as well as

$$
\left\|D^{2} u\right\|_{\infty} \leq M\|\phi\|_{C_{b}^{\beta}}
$$

for a constant $M$.

We easily get the extension

Theorem 1.13. Let $L_{t}, 0 \leq t \leq T$ be a d-dimensional truncated $\alpha$-stable process for $\alpha \in(1,2)$ and $d \geq 2$. Require that $\varphi \in C\left([0, T], C_{b}^{\beta}\left(\mathbb{R}^{d}\right)\right)$ for $\beta \in(0,1)$ with $\alpha+\beta>2$. Then there exists a $u \in C\left([0, T], C_{b}^{1, \beta}\left(\mathbb{R}^{d}\right)\right) \cap C\left([0, T], C_{b}^{2}\left(\mathbb{R}^{d}\right)\right) \cap C^{1}\left([0, T], C_{b}\left(\mathbb{R}^{d}\right)\right)$ satisfying the backward Kolmogorov equation

$$
\begin{aligned}
\frac{\partial u}{\partial t}+b \cdot \nabla u+\mathcal{L} u & =-\varphi \text { on }[0, T], \\
\left.u\right|_{t=T} & =0 .
\end{aligned}
$$

Moreover

$$
\|D u\|_{C_{b}^{\beta}} \leq C(T)\|\varphi\|_{C_{b}^{\beta}}
$$

where

$$
C(T) \longrightarrow 0 \text { for } T \searrow 0
$$

as well as

$$
\left\|D^{2} u\right\|_{\infty} \leq M\|\varphi\|_{C_{b}^{\beta}}
$$

for a constant $M$.

To be able to use the compactness criterion introduced above, we then define a self adjoint operator $A$ on $L^{2}((0, \tau)) \otimes L^{2}(\nu)$ (for fixed $\tau>0$ ) which admits a compact inverse $A^{-1}$ : $L^{2}((0, \tau)) \otimes L^{2}(\nu) \longrightarrow L^{2}((0, \tau)) \otimes L^{2}(\nu)$. More precisely, the operator $A$ is constructed as follows: Let the function $p$ (potential) be given by

$$
p(t, x)= \begin{cases}\frac{1}{|x|^{d+\alpha+\delta}} & , \text { if }|x| \leq \frac{1}{2} \\ \frac{2^{d+\alpha}}{(1-|x|)^{1 / 2}} & , \text { if } \frac{1}{2}<|x|<1\end{cases}
$$

for some $\delta>0$ such that $\alpha+\delta<2$. Consider now for fixed $\tau>0$ the symmetric form $\mathcal{E}$ on 
$L^{2}((0, \tau)) \otimes L^{2}(\nu)$ defined as

$$
\begin{aligned}
\mathcal{E}(f, g)= & \int_{0}^{\tau} \int_{0}^{\tau} \int_{|y|<1} \int_{|x|<1} \frac{\left(f\left(t_{1}, x\right)-f\left(t_{2}, y\right)\right)\left(g\left(t_{1}, x\right)-g\left(t_{2}, y\right)\right)}{\left(\left|t_{1}-t_{2}\right|+|x-y|\right)^{d+1+2 s}} d x d y d t_{1} d t_{2} \\
& +\int_{0}^{\tau} \int_{|x|<1} p(t, x) f(t, x) g(x) d x d t
\end{aligned}
$$

for functions $f, g$ in the (dense) domain $D(\mathcal{E}) \subset L^{2}((0, \tau)) \otimes L^{2}(\nu)$ and a fixed $s$ with

$$
0<s<\frac{1}{2}
$$

where

$$
\begin{aligned}
& D(\mathcal{E}) \\
: & =\left\{f:\|f\|_{L^{2}((0, \tau)) \otimes L^{2}(\nu)}^{2}+\int_{0}^{\tau} \int_{0}^{\tau} \int_{|y|<1} \int_{|x|<1} \frac{\left|f\left(t_{1}, x\right)-f\left(t_{2}, y\right)\right|^{2}}{\left(\left|t_{1}-t_{2}\right|+|x-y|\right)^{d+1+2 s}} d x d y d t_{1} d t_{2}\right. \\
& \left.+\int_{0}^{\tau} \int_{|x|<1} p(t, x)|f(t, x)|^{2} d x d t<\infty\right\} .
\end{aligned}
$$

Then $\mathcal{E}$ is a positive symmetric closed form and we can find by Kato's first representation theorem a positive self adjoint operator $T_{\mathcal{E}}$ such that

$$
\mathcal{E}(f, g)=\left(f, T_{\mathcal{E}} g\right)_{L^{2}((0, \tau)) \otimes L^{2}(\nu)}
$$

for for all $g \in D\left(T_{\mathcal{E}}\right), f \in D(\mathcal{E})$. Further, since

$$
\mathcal{E}(f, f) \geq\|f\|_{L^{2}((0, \tau)) \otimes L^{2}(\nu)}^{2}
$$

for all $f \in D(\mathcal{E})$, that is the form $\mathcal{E}$ is bounded from below by a positive number, we also have that $D(\varepsilon)=D\left(T_{\varepsilon}^{1 / 2}\right)$. Let us now define the operator $A$ as

$$
A=T_{\mathcal{E}}^{1 / 2}
$$

We then show that $A$ has a discrete spectrum and a compact operator inverse $A^{-1}$.

We also need the following estimate with respect to the operator $A$ :

Lemma 1.14. Let $b \in C\left([0, T] ; C_{b}^{\beta}\left(\mathbb{R}^{d}\right)\right) \cap C\left([0, T], C_{b}^{\infty}\left(\mathbb{R}^{d}\right)\right)$. Further, let $X$. be the unique strong solution to (1.35) with respect to the drift coefficient $b$. Then for sufficiently small $T<\infty$ we have that

$$
E\left[\left\|A D_{.,}, X(\tau)\right\|_{L^{2}((0, \tau)) \otimes L^{2}(\nu)}^{2}\right] \leq K \exp \left(T M H_{1}\left(\|b\|_{C_{b}^{\beta}}^{2}\right)\right)
$$

for all $0<\tau \leq T$, where $K, M<\infty$ are constants independent of $b$ and where $H_{1}$ is $a$ non-negative continuous function given by

$$
H_{1}(y):=\frac{(y+1)^{2}}{\left(1-C^{2}(T) y\right)}, 0 \leq y<\frac{1}{C^{2}(T)} .
$$


for a constant $C(T)$ with $C(T) \longrightarrow 0$ as $T \searrow 0$.

Finally, from the above the compactness criterion is satisfied with the operator $A$ and we get;

Theorem 1.15. Suppose that $L_{t}, 0 \leq t \leq T$ is a d-dimensional truncated $\alpha$-stable process for $\alpha \in(1,2)$ and $d \geq 2$. Require that $b \in C_{b}^{\beta}\left(\mathbb{R}^{d}\right)$ for $\beta \in(0,1)$ such that $\alpha+\beta>2$. Then there exists for sufficiently small $T>0$ a unique strong solution $X$. to the $S D E$

$$
d X_{t}=b\left(X_{t}\right) d t+d L_{t}, 0 \leq t \leq T, X_{0}=x .
$$

Moreover, $X_{t}$ is Malliavin differentiable for all $0 \leq t \leq T$. 
Part I

Articles 


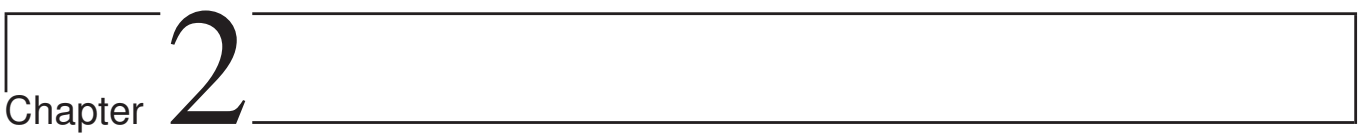

Optimal Stopping and Stochastic Control

Differential Games for Jump Diffusions

With Fouzia Baghery, Bernt Øksendal and Isabelle Turpin.

Published in Stochastics: An International Journal of Probability and Stochastic Processes. 


\section{$2.1 \quad$ Introduction}

Let $X(t)=X(t, \omega) \in[0, \infty) \times \Omega$ be a stochastic process on a filtered probability space $\left(\Omega, \mathcal{F},\left(\mathcal{F}_{t}\right)_{t \geq 0}, \mathbb{P}\right)$ representing the wealth of an investment at time $t$. The owner of the investment wants to find the optimal time for selling the investment. If we interpret "optimal" in the sense of "risk minimal", then the problem is to find a stopping time $\tau=\tau(\omega)$ which minimizes $\rho(X(\tau))$, where $\rho$ denotes a risk measure. If the risk measure $\rho$ is chosen to be a convex risk measure in the sense of Föllmer and Schied [2002] and (or) Frittelli and Gianin [2002], then it can be given the representation

$$
\rho(X)=\sup _{\mathbb{Q} \in \mathcal{N}}\left\{\mathbb{E}_{\mathbb{Q}}[-X]-\zeta(Q)\right\}
$$

for some set $\mathcal{N}$ of probability measures $\mathbb{Q} \ll \mathbb{P}$ and some convex "penalty" function $\zeta: \mathcal{N} \rightarrow \mathbb{R}$.

Using this representation the optimal stopping problem above gets the form

$$
\inf _{\tau \in \mathcal{T}}\left(\sup _{\mathbb{Q} \in \mathcal{N}}\left\{\mathbb{E}_{\mathbb{Q}}[-X(\tau)]-\zeta(Q)\right\}\right)
$$

where $\mathcal{T}$ is a given family of admissible $\mathcal{F}_{t^{-}}$stopping times. This may be regarded as an optimal stopping-stochastic control differential game.

In this paper we study this problem in a jump diffusion context. In Section 2.2 we formulate a general optimal stopping-stochastic control differential game problem in this context and we prove a general verification theorem for such games in terms of variational inequality-HamiltonJacobi-Bellman (VIHJB) equations. Then in Section 2.3 we apply the general results obtained in Section 2.2 to study the problem (2.2). By parametrizing the measures $\mathbb{Q} \in \mathcal{N}$ by a stochastic process $\theta(t, z)=\left(\theta_{0}(t), \theta_{1}(t, z)\right)$ we may regard (2.2) as a special case of the general stochastic differential game in Section 2.2. We use this to solve the problem in some special cases.

\subsection{General formulation}

In this section we put the problem in the introduction into a general framework of optimal stopping and stochastic control differential game for jump diffusions and we prove a verification theorem for the value function of such a game. We refer to Øksendal and Sulem [2007] for information about optimal stopping and stochastic control for jump diffusions. The following presentation follows Mataramvura and Øksendal [2008] closely.

Suppose the state $Y(t)=Y^{u}(t)=Y_{t}^{y, u}$ at time $t$ is given as the solution of a stochastic differential equation of the form

$$
\left\{\begin{array}{l}
d Y(t)=b\left(Y(t), u_{0}(t)\right) d t+\sigma\left(Y(t), u_{0}(t)\right) d B(t) \\
+\int_{\mathbb{R}_{0}^{k}} \gamma\left(Y\left(t^{-}\right), u_{1}(t, z), z\right) \tilde{N}(d t, d z) \\
Y(0)=y \in \mathbb{R}^{k}
\end{array}\right.
$$

Here $b: \mathbb{R}^{k} \times K \rightarrow \mathbb{R}^{k}, \sigma: \mathbb{R}^{k} \times K \rightarrow \mathbb{R}^{k \times k}$ and $\gamma: \mathbb{R}^{k} \times K \times \mathbb{R}^{k} \rightarrow \mathbb{R}^{k \times k}$ are given functions, $B(t)$ is a $k$-dimensional Brownian motion and $\tilde{N}(.,)=.\left(\tilde{N}_{1}(.,),. \ldots, \tilde{N}_{k}(.,).\right)$ are $k$ independent compensated Poisson random measures independent of $B($.$) , while K$ is a given subset of $\mathbb{R}^{p}$. For each $j=1, \ldots, k$ we have $\tilde{N}_{j}(d t, d z)=N_{j}(d t, d z)-\nu_{j}(d z) d t$, where $\nu_{j}$ is the Lévy measure (intensity measure) of the Poisson random measure $N_{j}(.,$.$) .$ 
We may regard $u(t, z)=\left(u_{0}(t), u_{1}(t, z)\right)$ as our control process, assumed to be càdlàg , $\mathcal{F}_{t}$-adapted and with values in $K \times K$ for a.a. $t, z, \omega$.

Thus $Y(t)=Y^{(u)}(t)$ is a controlled jump diffusion.

Let $f: \mathbb{R}^{k} \times K \rightarrow \mathbb{R}$ and $g: \mathbb{R}^{k} \rightarrow \mathbb{R}$ be given functions. Let $\mathcal{A}$ be a given set of controls contained in the set of $u=\left(u_{0}, u_{1}\right)$ such that (2.3) has a unique strong solution and such that

$$
\mathbb{E}^{y}\left[\int_{0}^{\tau_{\mathcal{S}}}|f(Y(t), u(t))| d t\right]<\infty
$$

(where $\mathbb{E}^{y}$ denotes expectation when $Y(0)=y$ ) where

$$
\tau_{\mathcal{S}}=\inf \{t>0 ; Y(t) \notin \mathcal{S}\} \quad \text { (the bankruptcy time) }
$$

is the first exit time of a given open solvency set $\mathcal{S} \subset \mathbb{R}^{k}$. We let $\mathcal{T}$ denote the set of all stopping times $\tau \leq \tau_{\mathcal{S}}$. We assume that

$$
\left\{g^{-}(Y(\tau))\right\}_{\tau \in \mathcal{T}} \quad \text { is uniformly integrable. }
$$

Note that $Y^{(u)}(t)$ is quasi-left continuous, in the sense that for each given $\tau \in \mathcal{T}$ we have

$$
\lim _{t \rightarrow \tau^{-}} Y^{(u)}(t)=Y^{(u)}(\tau)
$$

see Jacod and Shiryaev [2003], Proposition I. 2.26 and Proposition I. 3.27.

For $\tau \in \mathcal{T}$ and $u \in \mathcal{A}$ we define the performance functional $J^{\tau, u}(y)$ by

$$
J^{\tau, u}(y)=\mathbb{E}^{y}\left[\int_{0}^{\tau} f(Y(t), u(t)) d t+g(Y(\tau))\right]
$$

(we interpret $g(Y(\tau))$ as 0 if $\tau=\infty$ ).

We regard $\tau$ as the "control" of player number 1 and $u$ as the control of player number 2, and consider the stochastic differential game to find the value function $\Phi$ and an optimal pair $\left(\tau^{*}, u^{*}\right) \in \mathcal{T} \times \mathcal{A}$ such that

$$
\Phi(y)=\inf _{u \in \mathcal{A}}\left(\sup _{\tau \in \mathcal{T}} J^{\tau, u}(y)\right)=J^{\tau^{*}, u^{*}}(y)
$$

We restrict ourselves to Markov controls $u=\left(u_{0}, u_{1}\right)$, i.e. we assume that $u_{0}(t)=\bar{u}_{0}(Y(t))$ and $u_{1}(t)=\bar{u}_{1}(Y(t), z)$ for some functions $\bar{u}_{0}: \mathbb{R}^{k} \rightarrow K, \bar{u}_{1}: \mathbb{R}^{k} \times \mathbb{R}^{k} \rightarrow K$. For simplicity of notation we will in the following not distinguish between $u_{0}$ and $\bar{u}_{0}, u_{1}$ and $\bar{u}_{1}$.

When the control $u$ is Markovian the corresponding process $Y^{(u)}(t)$ becomes a Markov pro- 
cess, with generator $A^{u}$ given by

$$
\begin{aligned}
A^{u} \varphi(y)= & \sum_{i=1}^{k} b_{i}\left(y, u_{0}(y)\right) \frac{\partial \varphi}{\partial y_{i}}(y) \\
+ & \frac{1}{2} \sum_{i, j=1}^{k}\left(\sigma \sigma^{t}\right)_{i j}\left(y, u_{0}(y)\right) \frac{\partial^{2} \varphi}{\partial y_{i} \partial y_{j}}(y) \\
+ & \sum_{j=1}^{k} \int_{\mathbb{R}}\left\{\varphi \left(y+\gamma^{(j)}\left(y, u_{1}(y, z), z\right)-\varphi(y)\right.\right. \\
& \left.-\nabla \varphi(y) \cdot \gamma^{(j)}\left(y, u_{1}(y, z), z\right)\right\} \nu_{j}(d z) ; \quad \varphi \in \mathbb{C}^{2}\left(\mathbb{R}^{k}\right) .
\end{aligned}
$$

Here $\nabla \varphi=\left(\frac{\partial \varphi}{\partial y_{1}}, \ldots, \frac{\partial \varphi}{\partial y_{k}}\right)$ is the gradient of $\varphi$ and $\gamma^{(j)}$ is column number $j$ of the $k \times k$ matrix $\gamma$.

We can now formulate the main result of this section:

Theorem 2.1. (Verification theorem for stopping-control games)

Suppose there exists a function $\varphi: \overline{\mathcal{S}} \rightarrow \mathbb{R}$ such that

(i) $\varphi \in \mathcal{C}^{1}(\mathcal{S}) \cap \mathrm{e}(\bar{S})$

(ii) $\varphi \geq g$ on $\mathcal{S}$

Define

$$
D=\{y \in \mathcal{S} ; \varphi(y)>g(y)\} \quad \text { (the continuation region). }
$$

Suppose, with $Y(t)=Y^{(u)}(t)$,

(iii) $\mathbb{E}^{y}\left[\int_{0}^{\tau_{S}} \chi_{\partial D}(Y(t)) d t\right]=0$ for all $u \in \mathcal{A}$

(iv) $\partial D$ is a Lipschitz surface

(v) $\varphi \in \mathfrak{C}^{2}(\mathcal{S} \backslash \partial D)$, with locally bounded derivatives near $\partial D$

(vi) there exists $\hat{u} \in \mathcal{A}$ such that

$$
A^{\hat{u}} \varphi(y)+f(y, \hat{u}(y))=\inf _{u \in \mathcal{A}}\left\{A^{u} \varphi(y)+f(y, u(y))\right\}\left\{\begin{array}{l}
=0, \text { for } y \in D, \\
\leq 0, \text { for } y \in \mathcal{S} \backslash D .
\end{array}\right.
$$

(vii) $\mathbb{E}^{y}\left[|\varphi(Y(\tau))|+\int_{0}^{\tau}\left|A^{u} \varphi(Y(t))\right| d t\right]<\infty$, for all $\tau \in \mathcal{T}$ and all $u \in \mathcal{A}$.

For $u \in \mathcal{A}$ define

$$
\tau_{D}=\tau_{D}^{(u)}=\inf \left\{t>0 ; Y^{(u)}(t) \notin D\right\}
$$

and, in particular,

$$
\hat{\tau}=\tau_{D}^{(\hat{u})}=\inf \left\{t>0 ; Y^{(\hat{u})}(t) \notin D\right\} .
$$

(viii) Suppose that the family $\left\{\varphi(Y(\tau)) ; \tau \in \mathcal{T}, \tau \leq \tau_{D}\right\}$ is uniformly integrable, for each $u \in \mathcal{A}, y \in \mathcal{S}$.

Then $\varphi(y)=\Phi(y)$ and $(\hat{\tau}, \hat{u}) \in \mathcal{T} \times \mathcal{A}$ is an optimal pair, in the sense that

$$
\Phi(y)=\inf _{u}\left(\sup _{\tau} J^{\tau, u}(y)\right)=\sup _{\tau} J^{\tau, \hat{u}}(y)=J^{\hat{\gamma}, \hat{u}}(y)=\varphi(y)=\inf _{u} J^{\tau_{D}, u}(y)=\sup _{\tau}\left(\inf _{u} J^{\tau, u}(y)\right) .
$$

Proof. Choose $\tau \in \mathcal{T}$ and let $\hat{u} \in \mathcal{A}$ be as in (vi). By an approximation argument (see Theorem 3.1 in Øksendal and Sulem [2007]) we may assume that $\varphi \in \mathcal{C}^{2}(\mathcal{S})$. Then by the Dynkin formula 
(see Theorem 1.24 in Øksendal and Sulem [2007]) and (vi) we have, with $\hat{Y}=Y^{(\hat{u})}$

$$
\begin{aligned}
\mathbb{E}^{y}\left[\varphi\left(\hat{Y}\left(\tau_{m}\right)\right]\right. & =\varphi(y)+\mathbb{E}^{y}\left[\int_{0}^{\tau_{m}} A^{\hat{u}} \varphi(\hat{Y}(t)) d t\right] \\
& \leq \varphi(y)-\mathbb{E}^{y}\left[\int_{0}^{\tau_{m}} f(\hat{Y}(t), \hat{u}(t)) d t\right]
\end{aligned}
$$

where $\tau_{m}=\tau \wedge m ; m=1,2, \ldots$.

Letting $m \rightarrow \infty$ this gives, by (2.4), (2.6), (vii), (i) and the Fatou Lemma,

$$
\begin{aligned}
\varphi(y) & \geq \liminf _{m \rightarrow \infty} \mathbb{E}^{y}\left[\int_{0}^{\tau_{m}} f(\hat{Y}(t), \hat{u}(t)) d t+\varphi\left(\hat{Y}\left(\tau_{m}\right)\right)\right] \\
& \geq \mathbb{E}^{y}\left[\int_{0}^{\tau} f(\hat{Y}(t), \hat{u}(t)) d t+g\left(\hat{Y}(\tau) \chi_{\{\tau<\infty\}}\right]=J^{\tau, \hat{u}}(y) .\right.
\end{aligned}
$$

Since this holds for all $\tau$ we have

$$
\varphi(y) \geq \sup _{\tau} J^{\tau, \hat{u}}(y) \geq \inf _{u}\left(\sup _{\tau} J^{\tau, u}(y)\right), \text { for all } u \in \mathcal{A} .
$$

Next, for given $u \in \mathcal{A}$ define, with $Y(t)=Y^{(u)}(t)$,

$$
\tau_{D}=\tau_{D}^{u}=\inf \{t>0 ; Y(t) \notin D\} .
$$

Choose a sequence $\left\{D_{m}\right\}_{m=1}^{\infty}$ of open sets such that $\bar{D}_{m}$ is compact, $\bar{D}_{m} \subset D_{m+1}$ and $D=\bigcup_{m=1}^{\infty} D_{m}$

and define

$$
\tau_{D}(m)=m \wedge \inf \left\{t>0 ; Y(t) \notin D_{m}\right\} .
$$

By the Dynkin formula we have, by (vi), for $m=1,2, \ldots$,

$$
\begin{aligned}
\varphi(y) & =\mathbb{E}^{y}\left[-\int_{0}^{\tau_{D}(m)} A^{u} \varphi(Y(t)) d t+\varphi\left(Y\left(\tau_{D}(m)\right)\right)\right] \\
& \leq \mathbb{E}^{y}\left[\int_{0}^{\tau_{D}(m)} f(Y(t), u(t)) d t+\varphi\left(Y\left(\tau_{D}(m)\right)\right] .\right.
\end{aligned}
$$

By the quasi-left continuity of $Y($.$) (see Jacod and Shiryaev [2003], Proposition I. 2. 26$ and Proposition I. 3. 27), we get

$$
Y\left(\tau_{D}(m)\right) \rightarrow Y\left(\tau_{D}\right) \text { a.s. } \quad \text { as } m \rightarrow \infty .
$$

Therefore, if we let $m \rightarrow \infty$ in (2.15) we get

$$
\varphi(y) \leq \mathbb{E}^{y}\left[\int_{0}^{\tau_{D}} f(Y(t), u(t)) d t+g\left(Y\left(\tau_{D}\right)\right)\right]=J^{\tau_{D}, u}(y) .
$$


Since this holds for all $u \in \mathcal{A}$ we get

$$
\varphi(y) \leq \inf _{u} J^{\tau_{D}, u}(y) \leq \sup _{\tau}\left(\inf _{u} J^{\tau, u}(y)\right)
$$

In particular, applying this to $u=\hat{u}$ we get equality, i.e.

$$
\varphi(y)=J^{\hat{\tau}, \hat{u}}(y)
$$

Combining (2.14), (2.16) and (2.17) we obtain

$$
\begin{aligned}
\inf _{u}\left(\sup _{\tau} J^{\tau, u}(y)\right) \leq \sup _{\tau} J^{\tau, \hat{u}}(y) \leq \varphi(y)=J^{\hat{r}, \hat{u}}(y)=\varphi(y) \\
\leq \inf _{u} J^{\tau_{D}, u}(y) \leq \sup _{\tau}\left(\inf _{u} J^{\tau, u}(y)\right) \leq \inf _{u}\left(\sup _{\tau} J^{\tau, u}(y)\right) .
\end{aligned}
$$

Since we always have

$$
\sup _{\tau}\left(\inf _{u} J^{\tau, u}(y)\right) \leq \inf _{u}\left(\sup _{\tau} J^{\tau, u}(y)\right)
$$

we conclude that we have equality everywhere in (2.18) and the proof is complete.

Remark 2.1. It is natural to ask if the value function in the above theorem is the unique viscosity solution of the corresponding HJB variational inequalities. This will be proved to be the case by some of us in a subsequent paper (work in progress).

\subsection{Viscosity Solutions}

Let the state, $Y(t)=Y^{u}(t)$, be given by equation (2.3), the performance functional by equation (2.7) and the value function by equation (2.8). In the following we will assume that the functions $b, \sigma, \gamma, f, g$ are continuous with respect to $(y, u)$. Further, the following standard assumptions are adopted; there exists $C>0, \alpha: \mathbb{R}^{k} \rightarrow \mathbb{R}^{k}$ with $\int \alpha^{2}(z) \nu(d z)<\infty$ such that for all $x, y \in \mathbb{R}^{k}$, $z \in \mathbb{R}^{k}$ and $u \in K$,

$$
\begin{aligned}
& \text { A1. }|b(x, u)-b(y, u)|+|\sigma(x, u)-\sigma(y, u)|+|f(x, u)-f(y, u)|+|g(x)-g(y)| \leq C|x-y|, \\
& \text { A2. }|b(y, u)|+|\sigma(y, u)| \leq C(1+|y|), \\
& \text { A3. }|f(y, u)|+|g(y)| \leq C(1+|y|)^{m} \\
& \text { A4. }\left|\gamma\left(x, u_{1}, z\right)-\gamma\left(y, u_{1}, z\right)\right| \leq \alpha(z)|x-y|, \\
& \text { A5. }\left|\gamma\left(x, u_{1}, z\right)\right| \leq \alpha(z)(1+|x|) \text { and }\left|\gamma\left(x, u_{1}, z\right)\right| \mathbf{1}_{|z|<1} \leq C_{x}, C_{x} \in \mathbb{R} .
\end{aligned}
$$

Let us define a HJB variational inequality by

$$
\max \left\{\inf _{u \in K}\left[A^{u} \varphi(y)+f(y, u(y))\right], g(y)-\varphi(y)\right\}=0,
$$

and

$$
\varphi=g \text { on } \partial \mathcal{S} \text {. }
$$


where $A^{y} \varphi(y)$ is defined by equation (2.9).

Definition 2.2 (Viscosity solutions). A locally bounded function $\varphi \in U S C(\overline{\mathcal{S}})$ is called a viscosity subsolution of (2.20)-(2.21) in $\mathcal{S}$ if (2.21) holds and for each $\psi \in C_{0}^{2}(\mathcal{S})$ and each $y_{0} \in \mathcal{S}$ such that $\psi \geq \varphi$ on $\mathcal{S}$ and $\psi\left(y_{0}\right)=\varphi\left(y_{0}\right)$, we have

$$
\max \left\{\inf _{u \in K}\left[A^{u} \psi\left(y_{0}\right)+f\left(y_{0}, u\left(y_{0}\right)\right)\right], g\left(y_{0}\right)-\psi\left(y_{0}\right)\right\} \geq 0
$$

A function $\varphi \in L S C(\overline{\mathcal{S}})$ is called a viscosity supersolution of the (2.20)-(2.21) in $\mathcal{S}$ if (2.21) holds and for each $\psi \in C_{0}^{2}(\mathcal{S})$ and each $y_{0} \in \mathcal{S}$ such that $\psi \leq \varphi$ on $\mathcal{S}$ and $\psi\left(y_{0}\right)=\varphi\left(y_{0}\right)$, we have

$$
\max \left\{\inf _{u \in K}\left[A^{u} \psi\left(y_{0}\right)+f\left(y_{0}, u\left(y_{0}\right)\right)\right], g\left(y_{0}\right)-\psi\left(y_{0}\right)\right\} \leq 0
$$

Further, if $\varphi \in C\left([0, T] \times \mathbb{R}^{n}\right)$ is both a viscosity subsolution and a viscosity supersolution it is called a viscosity solution.

Proposition 2.3 (Dynamic programming principle). Let $\Phi$ be as in (2.8). Then we have

(i) $\forall h>0, \forall y \in \mathbb{R}^{k}$

$$
\Phi(y)=\sup _{\tau \in \mathcal{T}} \inf _{u \in \mathcal{A}} E^{y}\left[\int_{0}^{\tau \wedge h} f(Y(s), u(s)) d s+g\left(Y_{\tau}\right) \mathbf{1}_{\tau<h}+\Phi\left(Y_{h}\right) \mathbf{1}_{h \leq \tau}\right]
$$

(ii) Let $\varepsilon>0, y \in \mathbb{R}^{k}, u \in \mathcal{A}$ and define the stopping time

$$
\tau_{y, u}^{\varepsilon}=\inf \left\{0 \leq t \leq \tau_{s} ; \Phi\left(Y_{t}^{y, u}\right) \leq g\left(Y_{t}^{y, u}\right)+\varepsilon\right\} .
$$

Then, if $\tau_{u} \leq \tau_{y, u}^{\varepsilon}$, for all $u \in \mathcal{A}$, we have that:

$$
\Phi(y)=\inf _{u \in \mathcal{A}} E^{y}\left[\int_{0}^{\tau_{u}} f(Y(s)) d s+\Phi\left(Y_{\tau_{u}}^{y}\right)\right]
$$

Remark 2.2. Prop. 2.3 (i) is a consequence of Prop. 2.3 (ii) as observed in Krylov Krylov [1980] p135.

Proof. As the proof is long and complex it is postponed to Part 5.

Theorem 2.4. Under assumptions A1-A4, the value function $\Phi$ is a viscosity solution of (2.20)(2.21).

Proof. $\Phi$ is continuous according to the estimates of the moments of the jump diffusion state process (see Lemma 3.1 p.9 in Pham [1998]) and from Lipschitz condition A2 on $f$ and $g$ we get that

$$
\Phi(y)=g(y) \text { on } \partial S \text {. }
$$

We now prove that $\Phi$ is a subsolution of (2.20)-(2.21). Let $\psi \in C_{0}^{2}(\mathcal{S})$ and $y_{0} \in \mathcal{S}$ such that

$$
0=(\psi-\Phi)\left(y_{0}\right)=\min _{y}(\psi-\Phi)
$$


Define

$$
D=\{y \in \mathcal{S} \mid \Phi(y)>g(y)\} .
$$

If $y_{0} \notin D$ then $g\left(y_{0}\right)=\Phi\left(y_{0}\right)$ and hence (2.22) holds. Next suppose $y_{0} \in D$. Then we have by Proposition 3.2 for $\hat{\tau}=\tau_{D}$ and $h>0$ small enough:

$$
\Phi\left(y_{0}\right)=\inf _{u \in \mathcal{A}} E^{y_{0}}\left[\int_{0}^{h} f\left(Y^{y_{0}}(t), u(t)\right) d t+\Phi\left(Y^{y_{0}}(h)\right)\right] .
$$

From (2.24) we get

$$
0 \leq \inf _{u \in \mathcal{A}} E^{y_{0}}\left[\int_{0}^{h} f\left(Y^{y_{0}}(t), u(t)\right) d t+\psi\left(Y^{y_{0}}(h)\right)-\psi\left(y_{0}\right)\right] .
$$

By Itô 's formula we obtain that

$$
0 \leq \inf _{u \in \mathcal{A}} \frac{1}{h} E^{y_{0}}\left[\int_{0}^{h}\left[A^{u} \psi\left(Y_{t}^{y_{0}}\right)+f\left(Y^{y_{0}}(t), u(t)\right)\right] d t\right] .
$$

Using assumptions A1-A4 with estimates on the moments of a jump diffusion and by letting $h \rightarrow 0^{+}$, we have

$$
\inf _{u \in K}\left[A^{u} \psi\left(y_{0}\right)+f\left(y_{0}, u\left(y_{0}\right)\right)\right] \geq 0,
$$

and hence

$$
\max \left\{\inf _{u \in K}\left[A^{u} \psi\left(y_{0}\right)+f\left(y_{0}, u\left(y_{0}\right)\right)\right], g\left(y_{0}\right)-\psi\left(y_{0}\right)\right\} \geq 0 .
$$

This shows that $\Phi$ is a viscosity subsolution. The proof for supersolution is similar.

The problem of showing uniqueness of viscosity solution is not addressed in this paper but will be considered in a future article.

\subsection{Examples}

Let us look at some control problems where we include stopping times as one of the controls. We then apply the result of the previous section to find a solution. We will look at both a jump and a non-jump market.

Exemple 2.5 (Optimal Resource Extraction in a Worst Case Scenario). Let

$$
d P(t)=P(t)\left[\alpha d t+\beta d B(t)+\int_{\mathbb{R}_{0}} \gamma(z) \tilde{N}(d t, d z)\right] ; P(0)=y_{1}>0,
$$

where $\alpha, \beta$ are constants and $\gamma(z)$ is a given function such that $\int_{\mathbb{R}_{0}} \gamma^{2}(z) \nu(d z)<\infty$. Let $Q(t)$ be the amount of remaining resources at time $t$, and let the dynamics be described by

$$
d Q(t)=-u(t) Q(t) d t ; Q(0)=y_{2} \geq 0 .
$$

where $u(t)$ controls the consumption rate of the resource $Q(t)$, and $m$ is the maximum extraction 
rate. We let

$$
d Y(t)=\left\{\begin{array}{l}
d Y_{0}(t)=d t \\
d Y_{1}(t)=d P(t) ; P(0)=y_{1}>0 \\
d Y_{2}(t)=d Q(t) ; Q(0)=y_{2} \geq 0 \\
d Y_{3}(t)=-Y_{3}(t)\left[\theta_{0}(t) d B(t)+\int_{\mathbb{R}_{0}} \theta_{1}(t, z) \tilde{N}(d t, d z)\right] ; Y_{3}(0)=y_{3}>0
\end{array}\right.
$$

Let the running cost be given by $K_{0}+K_{1} u_{t}$ ( $K_{0}, K_{1} \geq 0$, constants). Then we let our performance functional be given by, with $\theta=\left(\theta_{0}, \theta_{1}\right)$,

$$
\begin{aligned}
& J^{\tau, u, \theta}\left(s, y_{1}, y_{2}, y_{3}\right) \\
& =E^{y}\left[\int_{0}^{\tau} e^{-\delta(s+t)}\left(u(t)\left(P(t) Q(t)-K_{1}\right)-K_{0}\right) Y_{3}(t) d t+e^{-\delta(s+\tau)}(M P(\tau) Q(\tau)-a) Y_{3}(\tau)\right]
\end{aligned}
$$

where $\delta>0$ is the discounting rate and $M>0, a>0$ are constants ( $a$ can be seen as a transaction cost). Our problem is to find $(\hat{\tau}, \hat{u}, \hat{\theta})$ in $\mathcal{T} \times U \times \Theta$ such that

$$
\Phi(y)=\Phi\left(s, y_{1}, y_{2}, y_{3}\right)=\sup _{u}\left[\inf _{\theta}\left(\sup _{\tau} J^{\tau, u, \theta}(y)\right)\right]=J^{\hat{\tau}, \hat{u}, \hat{\theta}}(y) .
$$

Then the generator of $Y^{u, \theta}$ is given by;

$$
\begin{aligned}
A^{u, \theta} \varphi(y) & =A^{u, \theta} \varphi\left(s, y_{1}, y_{2}, y_{3}\right)=\frac{\partial \varphi}{\partial s}+y_{1} \alpha \frac{\partial \varphi}{\partial y_{1}}-u y_{2} \frac{\partial \varphi}{\partial y_{2}}+\frac{1}{2} y_{1}^{2} \beta^{2} \frac{\partial^{2} \varphi}{\partial^{2} y_{1}}+\frac{1}{2} y_{3}^{2} \theta_{0}^{2} \frac{\partial^{2} \varphi}{\partial^{2} y_{3}} \\
& -y_{1} y_{3} \beta \theta_{0} \frac{\partial^{2} \varphi}{\partial y_{1} \partial y_{3}} \\
& +\int_{\mathbb{R}_{0}}\left\{\varphi\left(s, y_{1}+y_{1} \gamma(z), y_{2}, y_{3}-y_{3} \theta_{1}(z)\right)-\varphi\left(s, y_{1}, y_{2}, y_{3}\right)-y_{1} \gamma(z) \frac{\partial \varphi}{\partial y_{1}}+y_{3} \theta_{1}(z) \frac{\partial \varphi}{\partial y_{3}}\right\} \nu(d z) .
\end{aligned}
$$

We need to find a subset $D$ of $\mathcal{S}=\mathbb{R}_{+}^{4}=[0, \infty)^{4}$ and $\varphi\left(s, y_{1}, y_{2}, y_{3}\right)$ such that

$$
\begin{aligned}
& \varphi\left(s, y_{1}, y_{2}, y_{3}\right)=g\left(s, y_{1}, y_{2}, y_{3}\right):=e^{-\delta s}\left(M y_{1} y_{2}-a\right) y_{3}, \forall\left(s, y_{1}, y_{2}, y_{3}\right) \notin D \\
& \varphi\left(s, y_{1}, y_{2}, y_{3}\right) \geq e^{-\delta s}\left(M y_{1} y_{2}-a\right) y_{3}, \forall\left(s, y_{1}, y_{2}, y_{3}\right) \in \mathcal{S} \\
& A^{u, \theta} \varphi\left(s, y_{1}, y_{2}, y_{3}\right)+f\left(s, y_{1}, y_{2}, y_{3}, u\right):=A^{u, \theta} \varphi\left(s, y_{1}, y_{2}, y_{3}\right)+e^{-\delta s}\left(u\left(y_{1} y_{2}-K_{1}\right)-K_{0}\right) y_{3} \\
& \leq 0, \forall\left(s, y_{1}, y_{2}, y_{3}\right) \in \mathcal{S} \backslash D, \forall u \in[0, m] \\
& \sup _{u}\left[\inf _{\theta}\left\{A^{u, \theta} \varphi\left(s, y_{1}, y_{2}, y_{3}\right)+e^{-\delta s}\left(u\left(y_{1} y_{2}-K_{1}\right)-K_{0}\right) y_{3}\right\}\right]=0, \forall\left(s, y_{1}, y_{2}, y_{3}\right) \in D .
\end{aligned}
$$

Then

$$
\hat{\theta_{0}}=\frac{y_{1}}{y_{3}} \beta \frac{\varphi_{13}}{\varphi_{33}},
$$


is a minimizer of $\theta_{0} \mapsto A^{u, \theta} \varphi\left(s, y_{1}, y_{2}, y_{3}\right)$ where we are using the notation

$$
\varphi_{i j}=\frac{\partial^{2} \varphi}{\partial y_{j} \partial y_{i}}
$$

Let $\hat{\theta}_{1}(z)$ be the minimizer of $\theta_{1}(z) \mapsto A^{u, \theta} \varphi(y)$ and let $\hat{u}$ be the the maximizer of $u \mapsto A^{u, \theta} \varphi(y)+$ $f(y, u)$ i.e.

$$
u \mapsto A^{u, \theta} \varphi\left(s, y_{1}, y_{2}, y_{3}\right)+e^{-\delta s} u y_{3}\left(y_{1} y_{2}-K_{1}\right)-u y_{2} \varphi_{2}-y_{3} K_{0}
$$

Let us try a function on the form

$$
\varphi\left(s, y_{1}, y_{2}, y_{3}\right)=e^{-\delta s} F(w), \text { where } w=y_{1} y_{2} y_{3}
$$

Then

$$
\hat{u}= \begin{cases}m, & \text { if } w F^{\prime}(w)<w-y_{3} K_{1} \\ 0, & \text { otherwise, }\end{cases}
$$

and

$$
\hat{\theta}=\beta\left(1+\frac{F^{\prime}(w)}{F^{\prime \prime}(w) w}\right)
$$

Further, the first order condition for $\hat{\theta}_{1}(z)$ is

$$
\int_{\mathbb{R}_{0}}\left\{(1+\gamma(z)) F^{\prime}\left(w(1+\gamma(z))\left(1-\hat{\theta}_{1}(z)\right)\right)-F^{\prime}(w)\right\} \nu(d z)=0 .
$$

For $w F^{\prime}(w)<w-y_{3} K_{1}$ we have

$$
\begin{aligned}
& A^{\hat{u}, \hat{\theta}} e^{-\delta s} F\left(y_{1}, y_{2}, y_{3}\right)=-\delta e^{-\delta s} F(w)+w e^{-\delta s} \alpha F^{\prime}(w)-m w e^{-\delta s} F^{\prime}(w) \\
& +\frac{1}{2} w^{2} \beta^{2} F^{\prime \prime}(w) e^{-\delta s}+\frac{1}{2} w^{2} \beta^{2} F^{\prime \prime}(w) e^{-\delta s}\left(1+\left(\frac{F^{\prime}(w)}{F^{\prime \prime}(w) w}\right)^{2}+\frac{2 F^{\prime}(w)}{F^{\prime \prime}(w) w}\right) \\
& -w \beta^{2} e^{-\delta s}\left(F^{\prime}(w)+\frac{\left(F^{\prime}(w)\right)^{2}}{F^{\prime \prime}(w) w}+w F^{\prime \prime}(w)+F^{\prime}(w)\right) \\
& +e^{-\delta s} \int_{\mathbb{R}_{0}}\left\{F\left(w(1+\gamma(z))\left(1-\hat{\theta}_{1}(z)\right)\right)-F(w)-w \gamma(z) F^{\prime}(w)+\hat{\theta_{1}}(z) w F^{\prime}(w)\right\} \nu(d z) \\
& =-\delta e^{-\delta s} F(w)+w e^{-\delta s} \alpha F^{\prime}(w)-m w e^{-\delta s} F^{\prime}(w) \\
& +\beta^{2} e^{-\delta s}\left(-\frac{\left(F^{\prime}(w)\right)^{2}}{2 F^{\prime \prime}(w)}-w F^{\prime}(w)\right) \\
& +e^{-\delta s} \int_{\mathbb{R}_{0}}\left\{F\left(w(1+\gamma(z))\left(1-\hat{\theta}_{1}(z)\right)\right)-F(w)-w \gamma(z) F^{\prime}(w)+\hat{\theta_{1}}(z) w F^{\prime}(w)\right\} \nu(d z)
\end{aligned}
$$


We then need that if $w F^{\prime}(w)<w-y_{3} K_{1}$, then

$$
\begin{aligned}
& A^{\hat{u}, \hat{\theta}} F(w)+\left(m\left(y_{1} y_{2}-K_{1}\right)-K_{0}\right) y_{3}=-\delta F(w)+w \alpha F^{\prime}(w)-m w F^{\prime}(w) \\
& -\beta^{2}\left(\frac{\left(F^{\prime}(w)\right)^{2}}{2 F^{\prime \prime}(w)}+w F^{\prime}(w)\right) \\
& +\int_{\mathbb{R}_{0}}\left\{F\left(w(1+\gamma(z))\left(1-\hat{\theta}_{1}(z)\right)-F(w)-w \gamma(z) F^{\prime}(w)+\hat{\theta}_{1}(z) w F^{\prime}(w)\right\} \nu(d z)\right. \\
& +\left(m\left(y_{1} y_{2}-K_{1}\right)-K_{0}\right) y_{3}=0 .
\end{aligned}
$$

Similarly, if $w F^{\prime}(w) \geq w-y_{3} K_{1}$, then $\hat{u}=0$ and hence we must have

$$
\begin{aligned}
& A^{\hat{u}, \hat{\theta}} F(w)-K_{0} y_{3}=-\delta F(w)+w \alpha F^{\prime}(w) \\
& -\beta^{2}\left(\frac{\left(F^{\prime}(w)\right)^{2}}{2 F^{\prime \prime}(w)}+w F^{\prime}(w)\right) \\
& +\int_{\mathbb{R}_{0}}\left\{F\left(w(1+\gamma(z))\left(1-\hat{\theta}_{1}(z)\right)\right)-F(w)-w \gamma(z) F^{\prime}(w)+\hat{\theta}_{1}(z) w F^{\prime}(w)\right\} \nu(d z) \\
& -K_{0} y_{3}=0
\end{aligned}
$$

The continuation region $D$ gets the form

$$
D=\left\{\left(s, y_{1}, y_{2}, y_{3}\right): F(w)>\left(M y_{1} y_{2}-a\right) y_{3}\right\}
$$

Therefore we get the requirement

$$
F(w)=\left(M y_{1} y_{2}-a\right) y_{3}, \forall\left(s, y_{1}, y_{2}, y_{3}\right) \notin D .
$$

In light of this requirement and in order for $\varphi$ to be on the form (2.30) we see that we need $K_{0}, K_{1}$ and $a$ to be zero. Hence we let $K_{0}=K_{1}=a=0$ from now on. Then we need that $F$ satisfies the variational inequality

$$
\max \left\{A_{0}^{\hat{u}, \hat{\theta}} F(w)+\tilde{m} w, M w-F(w)\right\}=0, w>0,
$$

where

$$
\begin{aligned}
& A_{0}^{\hat{u}, \hat{\theta}} F(w)=-\delta F(w)+w \alpha F^{\prime}(w)-\tilde{m} w F^{\prime}(w)-\beta^{2}\left(\frac{\left(F^{\prime}(w)\right)^{2}}{2 F^{\prime \prime}(w)}+w F^{\prime}(w)\right) \\
& +\int_{\mathbb{R}_{0}}\left\{F\left(w(1+\gamma(z))\left(1-\hat{\theta}_{1}(z)\right)\right)-F(w)-w \gamma(z) F^{\prime}(w)+\hat{\theta_{1}}(z) w F^{\prime}(w)\right\} \nu(d z),
\end{aligned}
$$

with

$$
\tilde{m}:=m \chi_{(-\infty, 1)}\left(F^{\prime}(w)\right)
$$

The variational inequality (2.38) - (2.40) is hard to solve analytically, but it may be accessible by numerical methods.

Exemple 2.6 (Worst case scenario optimal control and stopping in a Lévy -market) . Let our 
dynamics be given by

$$
\begin{aligned}
d Y_{0}(t) & =d t ; & & Y_{0}(0)=s \in \mathbb{R} . \\
d Y_{1}(t) & \left.=\left(Y_{1}(t) \alpha(t)-u(t)\right) d t+Y_{1}(t) \beta\right) d B(t) & & \\
& +Y_{1}\left(t^{-}\right) \int_{\mathbb{R}} \gamma(s, z) \tilde{N}(d s, d z) ; & & Y_{1}(0)=y_{1}>0 . \\
d Y_{2}(t) & =-Y_{2}(t) \theta_{0}(t) d B(t)-Y_{2}(t) \int_{\mathbb{R}} \theta_{1}(s, z) \tilde{N}(d s, d z) ; & & Y_{2}(0)=y_{2}>0 .
\end{aligned}
$$

Solve

$$
\Phi(s, x)=\sup _{u}\left[\sup _{\tau}\left(\inf _{\theta_{0}, \theta_{1}} J^{\theta, u, \tau}(s, x)\right)\right]
$$

where

$$
J^{\theta, u, \tau}(s, x)=E^{x}\left[\int_{0}^{\tau} e^{-\delta(s+t)} \frac{u^{\lambda}}{\lambda} Y_{2}(t) d t\right]
$$

The interpretation of this problem is the following:

$Y_{1}(T)$ represents the size of the population (e.g. fish) when a harvesting strategy $u(t)$ is applied to it. The process $Y_{2}(t)$ represents the Radon-Nikodym derivative of a measure $Q$ with respect to $P$, i.e.

$$
Y_{2}(t)=\frac{d\left(Q \mid \mathcal{F}_{t}\right)}{d\left(P \mid \mathcal{F}_{t}\right)}=E\left[\frac{d Q}{d P} \mid \mathcal{F}_{t}\right] ; 0 \leq t \leq T
$$

This means that we can write

$$
\begin{aligned}
& J^{\theta, y, \tau}(s, x)=E^{x}\left[\int_{0}^{\infty} e^{-\delta(s+t)} \chi_{[0, \tau](t)} \frac{u^{\lambda}(t)}{\lambda} E\left[\frac{d Q}{d P} \mid \mathcal{F}_{t}\right] d t\right] \\
& =E_{Q}^{x}\left[\int_{0}^{\infty} e^{-\delta(s+t)} \frac{u^{\lambda}(t)}{\lambda} d t\right] .
\end{aligned}
$$

Hence $J^{\theta, y, \tau}$ represents the expected utility up to the stopping time $\tau$, measured in terms of a scenario (probability measure $Q$ ) chosen by the market. Therefore our problem may be regarded as a worst case scenario optimal harvesting/stopping problem. Alternatively, the problem may be interpreted as a risk minimizing optimal stopping and control problem. To see this, we use the following representation of a given convex risk measure $\rho$ :

$$
\rho(F)=\sup _{Q \in \mathcal{P}}\left\{E_{Q}[-F]-\varsigma(Q)\right\} ; F \in L^{\infty}(P),
$$

where $\mathcal{P}$ is the set of all measures $Q$ above and $\varsigma: \mathcal{P} \rightarrow \mathbb{R}$ is a given convex "penalty" function. If $\varsigma=0$ as above, the risk measure $\rho$ is called coherent. See P. Artzner and Heath [1999], Frittelli and Gianin [2002] and Föllmer and Schied [2002] . 
In this case our generator becomes

$$
\begin{aligned}
& A^{u, \theta} \varphi\left(s, y_{1}, y_{2}\right)=\frac{\partial \varphi}{\partial s}+\left(y_{1} \alpha-u\right) \frac{\partial \varphi}{\partial y_{1}}+\frac{1}{2} y_{1}^{2} \beta^{2} \frac{\partial^{2} \varphi}{\partial^{2} y_{1}} \\
& +\frac{1}{2} y_{2}^{2} \theta_{0}^{2} \frac{\partial^{2} \varphi}{\partial^{2} y_{2}}-y_{1} y_{2} \beta \theta_{0} \frac{\partial^{2} \varphi}{\partial y_{1} \partial y_{2}} \\
& +\int_{\mathbb{R}}\left[\varphi\left(s, y_{1}+y_{1} \gamma(s, z), y_{2}-y_{2} \theta_{1}(s, z)\right)-\varphi\left(s, y_{1}, y_{2}\right)-y_{1} \gamma(s, z) \frac{\partial \varphi}{\partial y_{1}}+y_{2} \theta_{1}(z) \frac{\partial \varphi}{\partial y_{2}}\right] \nu(d z) .
\end{aligned}
$$

and hence

$$
\begin{aligned}
& A^{u, \theta} \varphi\left(s, y_{1}, y_{2}\right)+f\left(s, y_{1}, y_{2}\right)=\frac{\partial \varphi}{\partial s}+\left(y_{1} \alpha-u\right) \frac{\partial \varphi}{\partial y_{1}}+\frac{1}{2} y_{1}^{2} \beta^{2} \frac{\partial^{2} \varphi}{\partial^{2} y_{1}} \\
& +\frac{1}{2} y_{2}^{2} \theta_{0}^{2} \frac{\partial^{2} \varphi}{\partial^{2} y_{2}}-y_{1} y_{2} \beta \theta_{0} \frac{\partial^{2} \varphi}{\partial y_{1} \partial y_{2}} \\
& +\int_{\mathbb{R}}\left[\varphi\left(s, y_{1}+y_{1} \gamma(s, z), y_{2}-y_{2} \theta_{1}(s, z)\right)-\varphi\left(s, y_{1}, y_{2}\right)-y_{1} \gamma(s, z) \frac{\partial \varphi}{\partial y_{1}}+y_{2} \theta_{1}(z) \frac{\partial \varphi}{\partial y_{2}}\right] \nu(d z) \\
& +e^{-\delta s} \frac{u^{\lambda}}{\lambda} y_{2} .
\end{aligned}
$$

Imposing the first-order condition we get the following equations for the optimal control processes $\hat{\theta}_{0}, \hat{\theta}_{1}$ and $\hat{u}$ :

$$
\begin{gathered}
\hat{\theta_{0}}=\frac{y_{1}}{y_{2}} \beta \frac{\varphi_{12}}{\varphi_{22}}, \\
\int_{\mathbb{R}}\left\{\varphi_{2}\left(s, y_{1}+y_{1} \gamma(s, z), y_{2}-y_{2} \hat{\theta}_{1}(s, z)\right)-\varphi_{2}\left(s, y_{1}, y_{2}\right)\right\} \nu(d z)=0,
\end{gathered}
$$

and

$$
\hat{u}=\left(\frac{e^{\delta s} \varphi_{1}}{y_{2}}\right)^{\frac{1}{\lambda-1}}
$$

where $\varphi_{i}=\frac{\partial \varphi}{\partial y_{i}} ; i=1,2$. This gives

$$
\begin{aligned}
& A^{\hat{u}, \hat{\theta}} \varphi\left(s, y_{1}, y_{2}\right)+f\left(s, y_{1}, y_{2}, \hat{u}\right)=\frac{\partial \varphi}{\partial s}+\left(y_{1} \alpha-\left(e^{\delta s} \frac{\varphi_{1}}{y_{2}}\right) \frac{1}{\lambda-1}\right) \varphi_{1}+\frac{1}{2} y_{1}^{2} \beta^{2} \varphi_{11}-\frac{1}{2} y_{1}^{2} \beta^{2} \frac{\varphi_{12}^{2}}{\varphi_{22}} \\
& +\int_{\mathbb{R}}\left[\varphi\left(s, y_{1}+y_{1} \gamma(s, z), y_{2}-y_{2} \hat{\theta}_{1}(s, z)\right)-\varphi\left(s, y_{1}, y_{2}\right)-y_{1} \gamma(s, z) \frac{\partial \varphi}{\partial y_{1}}+y_{2} \hat{\theta}_{1}(z) \frac{\partial \varphi}{\partial y_{2}}\right] \nu(d z) \\
& +e^{-\delta s} \frac{\left(\frac{\phi_{1} e^{\delta s}}{y_{2}}\right)^{\frac{\lambda}{\lambda-1}}}{\lambda} y_{2} .
\end{aligned}
$$

Let us try a value function of the form

$$
\varphi\left(s, y_{1}, y_{2}\right)=e^{-\delta s} y_{1}^{\lambda} F\left(y_{2}\right),
$$


for some function $F$ (to be determined). Then

$$
\begin{gathered}
\hat{\theta}_{0}=\beta \frac{\lambda \beta F^{\prime}\left(y_{2}\right)}{y_{2} F^{\prime \prime}\left(y_{2}\right)}, \\
\int_{\mathbb{R}}\left\{(1+\gamma(s, z))^{\gamma} F^{\prime}\left(y_{2}-y_{2} \hat{\theta}_{1}(s, z)\right)-F^{\prime}\left(y_{2}\right)\right\} \nu(d z)=0,
\end{gathered}
$$

and

$$
\hat{u}=\left(\frac{F\left(y_{2}\right) \lambda}{y_{2}}\right)^{\frac{1}{\lambda-1}} y_{1}
$$

With $\hat{\theta_{1}}$ as in (2.44) put

$$
\begin{aligned}
A_{0}^{\hat{\theta}, \hat{u}} F\left(y_{2}\right) & =-\delta F\left(y_{2}\right)+\left(\alpha-\left(\frac{\lambda F\left(y_{2}\right)}{y_{2}}\right)^{\frac{1}{\lambda-1}}\right) \lambda F\left(y_{2}\right)+\frac{1}{2} \beta^{2} \lambda(\lambda-1) F\left(y_{2}\right)-\frac{1}{2} \beta^{2} \frac{F^{\prime 2}\left(y_{2}\right)}{F^{\prime \prime}\left(y_{2}\right)} \\
& +\frac{y_{2}}{\lambda}\left(\frac{\lambda F\left(y_{2}\right)}{y_{2}}\right)^{\frac{\lambda}{\lambda-1}}+\int_{\mathbb{R}}\left[(1+\gamma(z))^{\lambda} F\left(y_{2}-y_{2} \hat{\theta}_{1}(z)\right)\right. \\
& \left.-F\left(y_{2}\right)-\gamma(z) \lambda F\left(y_{2}\right)+y_{2} \hat{\theta}_{1}(z) F^{\prime}\left(y_{2}\right)\right] \nu(d z) .
\end{aligned}
$$

Thus we see that the problem reduces to the problem of solving a non-linear variational-integro inequality as follows:

Suppose there exits a process $\hat{\theta}_{1}(s, z)$ satisfying $(2.44)$ and a $C^{1}$-function $F: \mathbb{R}_{+} \rightarrow \mathbb{R}_{+}$such that if we put

$$
D=\left\{y_{2}>0 ; F\left(y_{2}\right)>0\right\}
$$

then $F \in C^{2}(D)$ and

$$
A_{0}^{\hat{\theta}, \hat{u}} F\left(y_{2}\right)=0 \text { for } y_{2} \in D .
$$

Then the function $\varphi$ given by (2.42) is the value function of the problem. The optimal control process are as in (2.43) - (2.45) and an optimal stopping time is

$$
\tau^{*}=\inf \left\{t>0 ; Y_{2}(t) \notin D\right\} .
$$

Exemple 2.7 (Risk minimizing optimal portfolio and stopping).

$$
\begin{array}{lrl}
d Y_{0}(t)=d t ; & Y_{0}(0)=s \in \mathbb{R} . \\
d Y_{1}(t)=Y_{1}(t)[(r+(\alpha-r) \pi(t)) d t+\beta \pi(t) d B(t)] ; & & Y_{1}(0)=y_{1}>0 \\
d Y_{2}(t)=-Y_{2}(t) \theta(t) d B(t) ; & & Y_{2}(0)=y_{2}>0
\end{array}
$$

where $r, \alpha$ and $\beta>0$ are constants. Solve

$$
\Phi(s, x)=\sup _{\pi}\left[\sup _{\tau}\left(\inf _{\theta} J^{\pi, \theta, \tau}\right)\right]
$$


where

$$
J^{\pi, \theta, \tau}(s, x)=E^{x}\left[e^{-\delta \tau} \lambda Y_{1}(\tau) Y_{2}(\tau)\right]
$$

where $0<\lambda \leq 1$ and $(1-\lambda)$ is a percentage transaction cost. The generator is

$$
\begin{aligned}
A^{\theta, \pi} \varphi\left(s, y_{1}, y_{2}\right)+f\left(s, y_{1}, y_{2}\right) & =\frac{\partial \varphi}{\partial s}+y_{1}(r+(\alpha-r) \pi) \frac{\partial \varphi}{\partial y_{1}}+\frac{1}{2} y_{1}^{2} \beta^{2} \pi^{2} \frac{\partial^{2} \varphi}{\partial^{2} y_{1}} \\
& +\frac{1}{2} y_{2}^{2} \theta^{2} \frac{\partial^{2} \varphi}{\partial^{2} y_{2}}-y_{1} y_{2} \beta \theta \pi \frac{\partial^{2} \varphi}{\partial y_{1} \partial y_{2}} .
\end{aligned}
$$

From the first order conditions we get that

$$
\hat{\pi}=\frac{(\alpha-r) \varphi_{1} \varphi_{22}}{y_{1} \beta^{2}\left(\varphi_{12}^{2}-\varphi_{11} \varphi_{22}\right)},
$$

and

$$
\hat{\theta}=\frac{(\alpha-r) \varphi_{1} \varphi_{12}}{\beta y_{2}\left(\varphi_{12}^{2}-\varphi_{11} \varphi_{22}\right)} .
$$

Let us try to put

$$
\varphi\left(s, y_{1}, y_{2}\right)=e^{-\delta s} \lambda y_{1} y_{2}
$$

Then we get

$$
\begin{gathered}
A^{\hat{\theta}, \hat{\pi}} \varphi\left(s, y_{1}, y_{2}\right)=y_{1} y_{2}(r-\delta), \\
\hat{\theta}=\frac{\alpha-r}{\beta} .
\end{gathered}
$$

and

$$
\hat{\pi}=0 .
$$

So if

$$
r-\delta \leq 0
$$

then $A^{\hat{\theta}, \hat{\pi}} \varphi \leq 0$ and the best is to stop immediately and $\varphi=\Phi$. If

$$
r-\delta>0
$$

then

$$
D=[0, T] \times \mathbb{R}^{k} \times \mathbb{R}^{k},
$$

so $\hat{\tau}=T$.

Remark 2.3. Note that the optimal value given in (2.53) for $\hat{\theta}$ corresponds to choosing the measure $Q$ defined by

$$
d Q(\omega)=Y_{2}(T) d P(\omega)
$$

to be an equivalent martingale measure for the underlying financial market $\left(S_{0}(t), S_{1}(t)\right)$ defined 
by

$$
\begin{aligned}
& d S_{0}(t)=r d t ; S_{0}(0)=0, \\
& d S_{1}(t)=S_{1}(t)[\alpha d t+\beta d B(t)] ; S_{1}(0)>0 .
\end{aligned}
$$

This illustrates that equivalent martingale measures often appear as solutions of stochastic differential games between the agent and the market. This was first proved in Øksendal and Sulem [2008] and subsequent in a partial information context in An and Øksendal [2008] and An et al. [2008].

\subsection{Proof of the Dynamical Programming Principle (Prop.} 2.3)

The Proposition 2.3 (ii) is proved by Krylov Krylov [1980] for diffusion processes and mixed strategies whereas Pham Pham [1998] has mentioned how to generalize it to this context of jump diffusions. Let us explain how it may be adapted to our case of stochastic differential games with optimal stopping and stochastic control for jump diffusions. First we need a Bellman's Principle for stochastic differential games. We here refer to Fleming and Sougadinis Fleming and Souganidis [1989] Theorem 1.6 and Biswas Biswas Theorem 2.2, whose proofs rest on the continuity of the value functions and the introduction of a restrictive class of admissible strategies. Next, to generalize the Dynamic Programming Principle to our optimal stopping and stochastic control differential games problem, we use the technique of randomized stopping developed by Krylov Krylov [1980] p. 36. For greater generality, we establish a version of the Dynamical Programming Principle whose Prop. 2.3 is a particular case.

\subsubsection{A general context}

\subsubsection{Dynamics}

For a fixed positive constant $T$ and $s \in[0, T)$, the state $Y(t)=Y_{t}^{s, y, u}$ where $0 \leq t \leq T-s$ is driven by

$$
\begin{aligned}
d Y(t)= & b(s+t, Y(t), u(t)) d t+\sigma(s+t, Y(t), u(t)) d B(t) \\
& +\int_{\mathbb{R}_{0}^{k}} \gamma\left(s+t, Y\left(t^{-}\right), u(t), z\right) \tilde{N}(d t, d z)
\end{aligned}
$$

with the initial condition

$$
Y(s)=y \in S .
$$

$b:[0, T] \times \mathbb{R}^{k} \times K \rightarrow \mathbb{R}^{k}, \sigma:[0, T] \times \mathbb{R}^{k} \times K \rightarrow \mathbb{R}^{k \times k}$ and $\gamma:[0, T] \times \mathbb{R}^{k} \times K \times \mathbb{R}^{k} \rightarrow \mathbb{R}^{k \times k}$ are given functions and verify the assumptions of regularity of the part 3 uniformly with respect to $t$ and are lipschitz continuous in the variable $t$ for all $(y, u) . B(t)$ and $\tilde{N}(.,$.$) are defined as$ in part 2 .

$u(t)$ is the control process assumed to be predictable and with values in $K$ for a.a. $t, \omega$. The set of controls is denoted by $M(s)$.

$$
\tau_{\mathcal{S}}=\inf \{0<t \leq T-s ; Y(t) \notin \mathcal{S}\}
$$

$\mathcal{T}_{s, T}$ denotes the set of all stopping times $\tau \leq \tau_{\mathcal{S}}$ 
For $\tau \in \mathcal{T}_{s, T}$ and $u \in M(s)$ the performance functional is

$$
J^{\tau, u}(s, y)=\mathbb{E}_{s, y}^{u}\left[\int_{0}^{\tau} f(s+t, Y(t), u(t)) e^{-\varphi_{t}} d t+g(\tau, Y(\tau)) e^{-\varphi_{\tau}}\right]
$$

where $\varphi_{t}^{s, y, u}=\int_{0}^{t} c^{u_{r}}\left(s+r, Y_{r}^{s, y, u}\right) d r, c:[0, T] \times \mathbb{R}^{k} \times K \rightarrow \mathbb{R}^{+}, f:[0, T] \times \mathbb{R}^{k} \times K \rightarrow \mathbb{R}$ and $g:[0, T] \times \mathbb{R}^{k} \rightarrow \mathbb{R}$ are given functions.

The value function is defined as

$$
\Phi(s, y)=\sup _{\tau \in \mathcal{T}_{s, T}} \inf _{u \in M(s)} J^{\tau, u}(s, y)
$$

\subsubsection{The canonical sample space}

We work in a canonical Wiener-Poisson space, following Biswas, Ishikawa and Kunita [2006] and Buckdahn et al. [2008]. For a constant $T$ and $0 \leq s<t \leq T$, let $\Omega_{s, t}^{1}$ be the standard Wiener space i.e. the set of all functions from $[s, t]$ to $\mathbb{R}^{k}$ starting from 0 and topologized by the sup-norm. We denote the corresponding Borel $\sigma$-algebra by $\mathcal{B}_{1}^{0}$ and let $P_{s, t}^{1}$ be the Wiener measure on $\left(\Omega_{s, t}^{1}, \mathcal{B}_{1}^{0}\right)$.

In addition, upon denoting $Q_{s, t}^{*}=[s, t] \times\left(\mathbb{R}^{k} \backslash 0\right)$, let $\Omega_{s, t}^{2}$ be the set of all $\mathbb{N} \bigcup\{\infty\}$-valued measures on $\left(Q_{s, t}^{*}, \mathcal{B}\left(Q_{s, t}^{*}\right)\right)$ where $\mathcal{B}\left(Q_{s, t}^{*}\right)$ is the usual Borel $\sigma$-algebra of $Q_{s, t}^{*}$. We denote $\mathcal{B}_{2}^{0}$ to be the smallest $\sigma$-algebra over $\Omega_{s, t}^{2}$ so that the mappings $q \in \Omega_{s, t}^{2} \mapsto q(A) \in \mathbb{N} \bigcup\{\infty\}$ are measurable for all $A \in \mathcal{B}\left(Q_{s, t}^{*}\right)$. Let the co-ordinate random measure $N_{s, t}$ be defined as $N_{s, t}(q, A)=q(A)$ for all $q \in \Omega_{s, t}^{2}, A \in \mathcal{B}\left(Q_{s, t}^{*}\right)$ and denote $P_{s, t}^{2}$ to be the probability measure on $\left(\Omega_{s, t}^{2}, \mathcal{B}_{2}^{0}\right)$ under which $N_{s, t}$ is a Poisson random measure with Lévy measure $\nu$ satisfying

$$
\int_{\mathbb{R} \backslash\{0\}} \min \left(|z|^{2}, 1\right) \nu(\mathrm{dz})<\infty
$$

Next, for very $0 \leq s<t \leq T$, we define $\Omega_{s, t}=\Omega_{s, t}^{1} \times \Omega_{s, t}^{2}, P_{s, t} \equiv P_{s, t}^{1} \otimes P_{s, t}^{2}$ and $\mathcal{B}_{s, t} \equiv$ $\mathcal{B}_{1}^{0} \times \bigotimes \mathcal{B}_{2}^{0}$ i.e. the completion of $\mathcal{B}_{1}^{0} \times \otimes \mathcal{B}_{2}^{0}$ with respect to the probability measure $P_{s, t}$. We will follow the convention that $\Omega_{t, T} \equiv \Omega_{t}$ and $\mathcal{B}_{t, T} \equiv \mathcal{F}_{t}$. A generic element of $\Omega_{t}$ is denoted by $\omega=\left(\omega_{1}, \omega_{2}\right)$, where $\omega_{i} \in \Omega_{t, T}^{i}$ for $i \in\{1,2\}$, and we define the coordinate functions

$$
W_{s}^{t}(\omega)=\omega_{1}(s) \text { and } \mathrm{N}^{\mathrm{t}}(\omega, \mathrm{A})=\omega_{2}(\mathrm{~A})
$$

for all $0 \leq t \leq s \leq T, \omega \in \Omega, A \in \mathcal{B}\left(Q_{t, T}^{*}\right)$. The process $W^{t}$ is a Brownian motion starting at $t$ and $N^{t}$ is a Poisson random measure on the probability space $\left(\Omega_{t}, \mathcal{F}_{t}, P_{t}\right)$, and they are independent. Also, for $t \in[0, T]$, the filtration $\mathcal{F}_{t, .}=\left(\mathcal{F}_{t, s}\right)_{s \in[t, T]}$ is defined as follows:

We make $\hat{\mathcal{F}}_{t, \text {. }}$ to be right-continuous and denote it by $\mathcal{F}_{t, .}^{+}$. Finally, we augment $\mathcal{F}_{t, \text {. }}^{+}$by $P_{t}$-null sets and call it $\mathcal{F}_{t, .}$. As and when it necessitates, we extend the filtration $\mathcal{F}_{t, \text {. for }} s<t$ by choosing $\mathcal{F}_{t, s}$ as the trivial $\sigma$ algebra augmented by $P_{t}$-null sets. We follow the convention that $\mathcal{F}_{t, T}=\mathcal{F}_{t}$. When the terminal time $T$ is replaced by another time point, say $\tau$, the filtration we have just described is denoted by $\mathcal{F}_{t . .}^{\tau}$.

Finally, note that the space $\Omega_{s, t}$ is defined as the product of canonical Wiener space and Poisson 
space. Therefore, for any $\tau \in(t, T)$, we can identify the probability space $\left(\Omega_{t}, \mathcal{F}_{t, .}, P_{t}\right)$ with $\left(\Omega_{t, \tau} \times \Omega_{\tau}, \mathcal{F}_{t, .}^{\tau} \otimes \mathcal{F}_{\tau, .}, P_{t, \tau} \otimes P_{\tau}\right)$ by the following bijection $\pi: \Omega_{t} \rightarrow \Omega_{t, \tau} \times \Omega_{\tau}$. For a generic element $\omega=\left(\omega_{1}, \omega_{2}\right) \in \Omega_{t}=\Omega_{t, T}^{1} \times \Omega_{t, T}^{2}$, we define

$$
\begin{gathered}
\omega^{t, \tau}=\left(\left.\omega_{1}\right|_{[t, \tau]},\left.\omega_{2}\right|_{[t, \tau]}\right) \in \Omega_{t, \tau} \\
\omega^{\tau, T}=\left(\left.\left(\omega_{1}-\omega_{1}(\tau)\right)\right|_{[t, \tau]},\left.\omega_{2}\right|_{[t, \tau]}\right) \in \Omega_{\tau, T} \\
\pi(\omega)=\left(\omega^{t, \tau}, \omega^{\tau, T}\right)
\end{gathered}
$$

The description of the inverse map $\pi^{-1}$ is also apparent from above.

\subsubsection{The general DPP}

Theorem 2.8. Let $s \in[0, T], y \in \mathbb{R}^{k}$ and let $\tau=\tau^{u} \in \mathcal{T}_{s, T}$ be defined for each $u \in \mathcal{M}(s)$. Then

$$
\begin{aligned}
\Phi(s, y)= & \sup _{\gamma \in \mathcal{T}_{s, T}} \inf _{u \in M(s)} \mathbb{E}_{s, y}^{u}\left[\int_{0}^{\tau \wedge \gamma} f^{u_{t}}\left(s+t, Y_{t}\right) e^{-\varphi_{t}} d t\right. \\
& \left.+g\left(s+\gamma, Y_{\gamma}\right) e^{-\varphi_{\gamma}} \chi_{\gamma \leq \tau}+\Phi\left(s+\tau, Y_{\tau}\right) e^{-\varphi_{\tau}} \chi_{\tau \leq \gamma}\right],
\end{aligned}
$$

where $f^{u_{t}}\left(s+t, Y_{t}\right)=f\left(s+t, Y_{t}, u_{t}\right)$.

This theorem is in fact a consequence of the more general following result. Let $\varepsilon>0$, we define:

$\tau_{s, y, u}^{\varepsilon}=\inf \left\{t \geq 0: \Phi\left(s+t, Y_{t}^{s, y, u}\right) \leq g\left(s+t, Y_{t}^{s, y, u}\right)+\varepsilon\right\}$.

Theorem 2.9. Let $s \in[0, T], y \in \mathbb{R}^{k}, u \in \mathcal{M}(s)$ and $\tau^{u} \in \mathcal{T}_{s, T}$. We are given a nonnegative process $r_{t}^{u}$, progressively mesurable and bounded. Then

$$
\begin{aligned}
\Phi(s, y) \geq & \inf _{u \in M(s)} \mathbb{E}_{s, y}^{u}\left[\int_{0}^{\tau}\left(f^{u_{t}}+r_{t} \Phi\right)\left(s+t, Y_{t}\right) e^{-\varphi_{t}-\int_{0}^{t} r_{p} d p} d t\right. \\
& \left.+\Phi\left(s+\tau, Y_{\tau}\right) e^{-\varphi_{\tau}-\int_{0}^{\tau} r_{p} d p}\right] .
\end{aligned}
$$

If $\tau^{u} \leq \tau_{y, u}^{\varepsilon}$ for some $\varepsilon>0$ and all $u \in \mathcal{M}(s)$, we have equality in (2.60).

Proof. (Theorem (5.2 to Theorem 5.1)

We write the right side of $(2.59)$ as $W_{1}(s, y)$ then

$$
\begin{aligned}
W_{1}(s, y) \geq & \inf _{u \in M(s)} \mathbb{E}_{s, y}^{u}\left[\int_{0}^{\tau \wedge \tau^{\varepsilon}} f^{u_{t}}\left(s+t, Y_{t}\right) e^{-\varphi_{t}} d t+g\left(s+\tau^{\varepsilon}, Y_{\tau^{\varepsilon}}\right) e^{-\varphi_{\tau^{\varepsilon}}} \chi_{\tau^{\varepsilon}<\tau}\right. \\
& \left.+\Phi\left(s+\tau, Y_{\tau}\right) e^{-\varphi_{\tau}} \chi_{\tau<\tau^{\varepsilon}}\right] .
\end{aligned}
$$

and from the inequality

$$
g\left(s+\tau^{\varepsilon}, Y_{\tau^{\varepsilon}}\right) \geq \Phi\left(s+\tau^{\varepsilon}, Y_{\tau^{\varepsilon}}\right)-\varepsilon,
$$

it follows that

$$
W_{1}(s, y) \geq \inf _{u \in M(s)} \mathbb{E}_{s, y}^{u}\left[\int_{0}^{\tau \wedge \tau^{\varepsilon}} f^{u t}\left(s+t, Y_{t}\right) e^{-\varphi_{t}} d t+\Phi\left(s+\tau \wedge \tau^{\varepsilon}, Y_{\tau \wedge \tau^{\varepsilon}}\right) e^{-\varphi_{\tau \wedge \tau^{\varepsilon}}}-\varepsilon\right](2.62)
$$


Since $\tau \wedge \tau^{\varepsilon} \leq \tau^{\varepsilon}$, by Theorem 5.2 for $r_{t}^{u}=0$, we have that the last lowerbound is equal to $\Phi(s, y)-\varepsilon$. Now let $\varepsilon$ tend to zero therefore $W_{1}(s, y) \geq \Phi(s, y)$.

On the other hand, $g(s, y) \leq \Phi(s, y)$ so that

$$
W_{1}(s, y) \leq \sup _{\gamma} \inf _{u \in M(s)} \mathbb{E}_{s, y}^{u}\left[\int_{0}^{\tau \wedge \gamma} f^{u_{t}}\left(s+t, Y_{t}\right) e^{-\varphi_{t}} d t+\Phi\left(s+\tau \wedge \gamma, Y_{\tau \wedge \gamma}\right) e^{-\varphi_{\tau \wedge \gamma}}\right]
$$

Let assume in Theorem 5.2 that $r_{t}^{u}=0$, we note that the last upper bound does not exceed $\Phi(s, y)$. Hence $W_{1}(s, y) \leq \Phi(s, y)$.

In order to investigate the proof of the Theorem 5.2 we introduce the case of stochastic differential games (see Biswas).

\subsubsection{The Stochastic Game}

\subsubsection{Context}

We introduce a two-player zero-sum stochastic differential game where the state is governed by controlled jump-diffusions. For $t \in[0, T-s]$

$$
\begin{aligned}
d Y(t)= & b(s+t, Y(t), u(t), v(t)) d t+\sigma(s+t, Y(t), u(t), v(t)) d B(t) \\
& +\int_{\mathbb{R}_{0}^{k}} \gamma\left(s+t, Y\left(t^{-}\right), u(t), v(t), z\right) \tilde{N}(d t, d z) ; \\
Y(s)= & y \in \mathbb{R}^{k} .
\end{aligned}
$$

Remark 2.10. For us and on the rest of the paper $b(t, y, u, v)=b(t, y, u), \sigma(t, y, u, v)=$ $\sigma(t, y, u)$ and $\gamma(t, y, u, v, z)=\gamma(t, y, u, z)$ so that $Y_{t}^{s, y, u, v}=Y_{t}^{s, y, u}=Y(t)$.

For convenience, we shall use the superscripts $u, v$ and the subscripts $s, y$ on the expectation sign to indicate expectation of quantites which depend on $s, y$ and strategies $u, v$. We introduce $f(t, y, u, v)=f^{u}(t, y)+v g(t, y) ; c^{u, v}(t, y)=c^{u}(t, y)+v$ and $\varphi_{t}^{s, y, u, v}=\int_{0}^{t} c^{u_{r}, v_{r}}\left(s+r, Y_{r}\right) d r$. We use the notation $\psi=(u, v)$ and define

$$
J_{n}^{\psi}(s, y)=\mathbb{E}_{s, y}^{\psi}\left[\int_{0}^{T-s} f\left(s+t, Y_{t}, u_{t}, v_{t}\right) e^{-\varphi_{t}} d t+g\left(T-s, Y_{T-s}\right) e^{-\varphi_{T-s}}\right],
$$

for strategie $v=\left(v_{t}\right)$ with values in $[0, n], n \in \mathbb{N}^{*}$.

\subsubsection{Admissible Controls and Strategies}

Definition 2.11. An admissible control process u(.) (resp. v(.)) for player I (resp. player II)

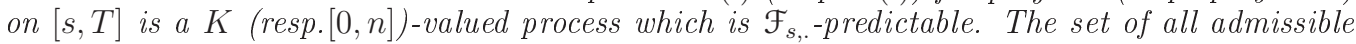
controls for player I (resp.II) is denoted by $\mathcal{M}(s)$ (resp. $\mathcal{N}(s)$ ).

We say the controls $u, \tilde{u} \in \mathcal{M}(s)$ are the same on $[s, t]$ and write $u \approx \tilde{u}$ on $[s, t]$ if $\mathbb{P}_{t}(u(r)=\tilde{u}(r)$ for a.e. $r \in[s, t])=1$. A similar convention is followed for members of $\mathcal{N}(s)$.

Definition 2.12. An admissible strategy $\alpha$ (resp. $\beta$ ) for player I (resp. II) is a mapping $\alpha: N(s) \rightarrow M(s)$ (resp. $\beta: M(s) \rightarrow N(s)$ ) such that if $v(.) \approx \tilde{v}($ resp.u $\approx \tilde{u})$ ) on $[s, t]$, then $\alpha[v] \approx \alpha[\tilde{v}]$ on $[s, t]$ for every $t \in[s, T]$ (resp. $\beta[u] \approx \beta[\tilde{u}]$ ). The set of admissible strategies for player $I$ (resp. II) on $[s, T]$ is denoted by $\Gamma_{n}(s)$ (resp. $\left.\Delta_{n}(s)\right)$. 
Remark 2.13. We will denote $\Gamma(s):=\bigcup_{n} \Gamma_{n}(s)$ and $\Delta(s):=\bigcup_{n} \Delta_{n}(s)$.

We set $B_{n}=K \times[0, n]$ and $\mathcal{B}_{n}$ the set of strategies. Let $\mathcal{R}_{n}$ be a set of nonnegative processes $\bar{r}_{t}$ which are progressively measurable with respect to $\left(\mathcal{F}_{t}\right)$ and such that $\bar{r}_{t}(\omega) \leq n$ for all $(t, \omega)$, $\mathcal{B}=\bigcup_{n} \mathcal{B}_{n}$ and $\mathcal{R}=\bigcup_{n} \mathcal{R}_{n}$. Each strategy $\psi \in \mathcal{B}_{n}$ is obviously a pair of processes $(u, \bar{v})$ with $u=\left(u_{t}\right) \in \mathcal{M}(s), \bar{v}=\left(\beta[u]_{t}\right) \in \Delta_{n}(s)$.

Definition 2.14. i) The lower value of the $S D G$ (2.64-2.65) with initial data $(s, y)$ is given by

$$
\underline{\Phi_{n}}(s, y):=\inf _{\alpha \in \Gamma_{n}(s)}\left(\sup _{v \in \mathcal{N}(s)} J_{n}(s, y, \alpha[v], v)\right)
$$

ii) The upper value of the $S D G(2.64-2.65)$ is

$$
\overline{\Phi_{n}}(s, y):=\sup _{\beta \in \Delta_{n}(s)}\left(\inf _{u \in \mathcal{M}(s)} J_{n}(s, y, u, \beta[u])\right) .
$$

\subsubsection{DPP for Stochastic Games}

Proposition 2.15. The upper and lower value functions are Lipschitz continuous in y, Hölder continuous in $t$ and verify $\left|\underline{\Phi_{n}}(s, y)\right|+\left|\overline{\Phi_{n}}(s, y)\right| \leq C(1+|y|)^{m}$.

Proposition 2.16. Let $s \in[0, T], \tau \in \mathcal{T}_{s, T}$, for every $y \in \mathbb{R}^{k}$,

$$
\begin{aligned}
\overline{\Phi_{n}}(s, y)= & \sup _{\beta \in \Delta_{n}(s)} \inf _{u \in \mathcal{M}(s)} E_{s, y}^{u, \beta}\left[\int_{0}^{\tau}\left(f^{u_{t}}+\beta[u]_{t} g\right)\left(s+t, Y_{t}\right) e^{-\varphi_{t}} d t\right. \\
& \left.+\overline{\Phi_{n}}\left(s+\tau, Y_{\tau}\right) e^{-\varphi_{\tau}}\right] \\
\underline{\Phi_{n}}(s, y)= & \inf _{\alpha \in \Gamma_{n}(s)} \sup _{v \in \mathcal{N}(s)} E_{s, y}^{\alpha, v}\left[\int_{0}^{\tau}\left(f^{\alpha[v]_{t}}+v_{t} g\right)\left(s+t, Y_{t}\right) e^{-\varphi_{t}} d t\right. \\
& \left.+\underline{\Phi_{n}}\left(s+\tau, Y_{\tau}\right) e^{-\varphi_{\tau}}\right] .
\end{aligned}
$$

Remark 2.17. (i) Thanks to comparison theorems for Isaacs equations and the inequality $H^{+} \leq H^{-}$where

$$
\begin{aligned}
& H^{+}=\inf _{u \in K} \sup _{v \in[0, n]}\left(A^{u} \varphi(t, y)+f(t, y, u, v)\right), \\
& H^{-}=\sup _{v \in[0, n]} \inf _{u \in K}\left(A^{u} \varphi(t, y)+f(t, y, u, v)\right),
\end{aligned}
$$

we have $\Phi_{n} \leq \overline{\Phi_{n}}$.

(ii) Under Isaac's condition, that is $H^{+}=H^{-}$, we have

$$
\underline{\Phi_{n}}(s, y)=\overline{\Phi_{n}}(s, y)
$$

This is true for instance:

- in the deterministic case, see Evans and Sougadinis [1984] 
- if the controls appear separated in both the dynamics and the payoff, see Bensoussan and Friedman [1974].

Proposition 2.18. (i) $\Phi(s, y)$ is Lipschitz continuous in $(s, y)$.

(ii) $\overline{\Phi_{n}}(s, y) \nearrow \Phi(s, y)$ uniformly on each set of the form $\{(s, y): s \in[0, T],|y| \leq R\}$.

\subsubsection{Proof of Theorem 5.2}

\subsubsection{Intermediate Results}

For more lightness we give all the intermediate results whose proofs are postponed in the next subsection. For $\psi=(u, v) \in K \times[0, n]$, let define

$$
G_{s, t}^{\psi} w(y)=\mathbb{E}_{s, y}^{\psi}\left[\int_{0}^{t-s} f^{\psi}\left(s+r, X_{r}\right) e^{-\varphi_{r}} d r+w\left(X_{t-s}\right) e^{-\varphi_{t-s}}\right]
$$

and

$$
G_{s, t} w(y)=\sup _{\psi} G_{s, t}^{\psi} w(y) .
$$

Lemma 2.19. Let $s_{0}<s_{1}<\ldots<s_{n}=T$. Then

$$
\overline{\Phi_{n}}\left(s_{0}, y\right) \geq G_{s_{0}, s_{1}} G_{s_{1}, s_{2}} \ldots G_{s_{n-1}, s_{n}} g(y) .
$$

Theorem 2.20. Let $s_{0}=s_{0}^{i}<s_{1}^{i}<\ldots<s_{n(i)}^{i}=T(i=1,2, \ldots), \max _{j}\left(s_{j+1}^{i}-s_{j}^{i}\right) \rightarrow 0$ for $i \rightarrow \infty$. Then

$$
\begin{aligned}
\overline{\Phi_{n}}\left(s_{0}, y\right) & =\lim _{i \rightarrow \infty} G_{s_{0}^{i}, s_{1}^{i}} G_{s_{1}^{i}, s_{2}^{i} \ldots G_{s_{n(i)-1}^{i}, s_{n(i)}^{i}}} g(y) \\
& =\sup _{i} G_{s_{0}^{i}, s_{1}^{i}} G_{s_{1}^{i}, s_{2}^{i} \ldots} G_{s_{n(i)-1}^{i}, s_{n(i)}^{i}} g(y) .
\end{aligned}
$$

Lemma 2.21. (a) Let $s \in[0, T], y \in \mathbb{R}^{k}, \psi=(u, v) \in M(s) \times \Delta_{n}(s)$ with $v=\beta[u]$ then the processes

$$
\delta_{t}^{\psi, s, y} \equiv \overline{\Phi_{n}}\left(s+t, Y_{t}^{u, s, y}\right) e^{-\varphi_{t}^{\psi, s, y}}-\mathbb{E}_{s, y}^{\psi}\left[\int_{t}^{T-s} f^{\psi_{r}}\left(s+r, Y_{r}\right) e^{-\varphi_{r}} d r+g\left(Y_{T-s}\right) e^{-\varphi_{T-s}} / F_{t}\right]
$$

and

$$
K_{t}^{\psi, s, y} \equiv \overline{\Phi_{n}}\left(s+t, Y_{t}^{u, s, y}\right) e^{-\varphi_{t}^{\psi, s, y}}+\int_{0}^{t} f^{\psi_{r}}\left(s+r, Y_{r}\right) e^{-\varphi_{r}} d r
$$

defined for $t \in[0, T-s]$ are supermartingales with respect to $\left\{F_{t}\right\}$, the first process being nonnegative (a.s.).

(b) $G_{s, t} \overline{\Phi_{n}}(t, y) \leq \overline{\Phi_{n}}(s, y)$ for $y \in \mathbb{R}^{k}, 0 \leq s \leq t \leq T$.

Lemma 2.22. Let $s \in[0, T], y \in \mathbb{R}^{k}, \psi=(u, \beta) \in M(s) \times \Gamma_{n}(s)$ then the process

$$
\overline{\Phi_{n}}\left(s+t, Y_{t}^{u, s, y}\right) e^{-\varphi_{t}^{\psi, s, y}}+\int_{0}^{t}\left[f^{u_{p}}+\beta\left[u_{p}\right] \overline{\Phi_{n}}\right]\left(s+p, Y_{p}^{u, s, y}\right) e^{-\varphi_{p}^{\psi, s, y}} d p
$$


defined for $t \in[0, T-s]$ is a continuous supermartingale.

Lemma 2.23. (a) Let $s \in[0, T], y \in \mathbb{R}^{k}$ and for each $u \in M(s)$ let $\tau^{u} \in \mathcal{T}_{s, T}, r_{t}^{u} \in \mathcal{R}$ be defined. Then

$\overline{\Phi_{n}}(s, y)=\inf _{u \in \mathcal{M}(s)} \mathbb{E}_{s, y}^{u}\left[\int_{0}^{\tau}\left[f^{u_{t}}+n\left(g-\overline{\Phi_{n}}\right)_{+}\right]\left(s+t, Y_{t}\right) e^{-\varphi_{t}-\int_{0}^{t} r_{p} d p} d t+\overline{\Phi_{n}}\left(s+\tau, Y_{\tau}\right) e^{-\varphi_{\tau}-\int_{0}^{\tau} r_{p} d p}\right]$

(b) Let $g_{n}=g \wedge \overline{\Phi_{n}}$. Then

$$
\overline{\Phi_{n}}(s, y)=\sup _{\tau \in \mathcal{T}_{s, T}} \inf _{u \in \mathcal{M}(s)} \mathbb{E}_{s, y}^{u}\left[\int_{0}^{\tau} f^{u_{t}}\left(s+t, Y_{t}\right) e^{-\varphi_{t}} d t+g_{n}\left(s+\tau, Y_{\tau}\right) e^{-\varphi_{\tau}}\right]
$$

Corollaire 2.24. Since $g_{n} \leq g, \overline{\Phi_{n}} \leq \Phi$.

Corollaire 2.25. (Consequence of Prop 5.9 and Lemma 5.15) Let $s \in[0, T], y \in \mathbb{R}^{k}, \psi=$ $(u, \bar{v}) \in \mathcal{B}$ then the process

$$
\rho_{t}^{\psi, s, y} \equiv \Phi\left(s+t, Y_{t}^{u, s, y}\right) e^{-\varphi_{t}^{\psi, s, y}}+\int_{0}^{t}\left[f^{u_{p}}+\bar{v}_{p} \Phi\right]\left(s+p, Y_{p}^{u, s, y}\right) e^{-\varphi_{p}^{\psi, s, y}} d p
$$

defined for $t \in[0, T-s]$ is a continuous supermartingale.

\subsubsection{Proofs}

The following result is given without demonstration, we refer to Krylov [1980] Lemma 2.14 p148.

Lemma 2.26. Let $s \in[0, T], 0 \leq t_{1} \leq t_{2} \leq T-s$ and $\psi \in \mathcal{M}(s) \times \Gamma(s)$ such that $\psi_{t}=\psi_{t_{1}}$ for $t \in\left[t_{1}, t_{2}\right)$. Let the continuous function $w(y)$ satisfying $|w(y)| \leq N(1+|y|)^{m}$. Then a.s.

$$
\mathbb{E}_{s, y}^{\psi}\left[\int_{t_{1}}^{t_{2}} f^{\psi_{t}}\left(s+t, Y_{t}\right) e^{-\varphi_{t}} d t+w\left(Y_{t_{2}}\right) e^{-\varphi_{t_{2}}} / \mathcal{F}_{t_{1}}\right]=e^{-\varphi_{t_{1}}^{\psi, s, y}} G_{s+t_{1}, s+t_{2}}^{\psi_{t_{1}}} w\left(Y_{t_{1}}^{\psi_{t_{1}}}\right) .
$$

Proof. (Lemme 5.15) By Lemma 5.14 (a),

$$
K_{t}^{\psi, s, y}=\overline{\Phi_{n}}\left(s+t, Y_{t}^{\psi, s, y}\right) e^{-\varphi_{t}^{\psi, s, y}}+\int_{0}^{t} f^{\psi_{p}}\left(s+p, Y_{p}\right) e^{-\varphi_{p}^{\psi, s, y}} d p
$$

is a supermartingale. In particular for $\psi=(u, 0)$,

$$
\overline{\Phi_{n}}\left(s+t, Y_{t}^{u, s, y}\right) e^{-\varphi_{t}^{u, s, y}}+\int_{0}^{t} f^{u_{p}}\left(s+p, Y_{p}^{u, s, y}\right) e^{-\varphi_{p}^{u, s, y}} d p
$$

is a supermartingale. Applying the lemma from Appendix 2 in Krylov [1980] with $\Phi_{t}=$ $e^{-\int_{0}^{t} \beta[u]_{s} d s}$ thus completing the proof.

Proof. (Lemme 5.12)

We introduce $w_{i}(y)=G_{s_{i}, s_{i+1}} \ldots G_{s_{n-1}, s_{n}} g(y), i=0, \ldots, n-1$ and $w_{n}(y)=g(y)$. Let $\varepsilon>$ 0, by the Theorem 2.2 (Chap. 3) in Krylov [1980] and the assumption (A3), we deduce the 
continuity of $w_{n-1}$ and that $\left|w_{n-1}(y)\right| \leq N(1+|y|)^{m}$. Arguing in the same way, we convince ourselves that all the functions $w_{i}(y)$ are continuous. Furthermore $w_{i}(y)=G_{s_{i}, s_{i+1}} w_{i+1}(y)=$ $\sup G_{s_{i}, s_{i+1}}^{\psi} w_{i+1}(y), i=0, \ldots, n-1$. By the corollary 2.8 in Krylov [1980] p145, the functions $G_{s_{i}, s_{i+1}}^{\psi} w_{i+1}(y)$ are continuous with respect to $\psi$ and $y$. Then for all $y \in \mathbb{R}^{k}$ there exists a Borel function $\beta_{i}^{y}$ such that

$$
w_{i}(y) \leq G_{s_{i}, s_{i+1}}^{u, \beta_{i}^{y}} w_{i+1}(y)+\varepsilon, \forall u \in \mathcal{M}(s) .
$$

We construct a strategy $\psi_{t}^{\varepsilon}=\left(u_{t}^{\varepsilon}, v_{t}^{\varepsilon}\right)$ by the following way: $u_{t}^{\varepsilon}=u_{i}^{\varepsilon} \in K$ and $v_{t}^{\varepsilon}=\beta\left[u_{i}^{\varepsilon}\right]$ for $t \in\left[s_{i}, s_{i+1}\right)$. Then $\psi_{t}^{\varepsilon}$ is admissible and by the lemma 5.19 ,

$\mathbb{E}_{s_{0}, y}^{\psi^{\varepsilon}}\left[\int_{s_{i}-s_{0}}^{s_{i+1}-s_{0}} f^{\psi_{t}}\left(s_{0}+t, Y_{t}\right) e^{-\varphi_{t}} d t+w_{i+1}\left(Y_{s_{i+1}-s_{0}}\right) e^{-\varphi_{s_{i+1}-s_{0}}}\right]=\mathbb{E}_{s_{0}, y}^{\psi^{\varepsilon}} e^{-\varphi_{s_{i}-s_{0}}} G_{s_{i}, s_{i+1}}^{\psi^{\varepsilon}} w_{i+1}\left(Y_{s_{i}-s_{0}}\right)$.

Thus this result combining with (2.72) yields

$$
\mathbb{E}_{s_{0}, y}^{\psi^{\varepsilon}}\left[\int_{s_{i}-s_{0}}^{s_{i+1}-s_{0}} f^{\psi_{t}}\left(s_{0}+t, Y_{t}\right) e^{-\varphi_{t}} d t+w_{i+1}\left(Y_{s_{i+1}-s_{0}}\right) e^{-\varphi_{s_{i+1}-s_{0}}}\right] \geq \mathbb{E}_{s_{0}, y}^{\psi^{\varepsilon}}\left(e^{-\varphi_{s_{i}-s_{0}}} w_{i}\left(Y_{s_{i}-s_{0}}\right)\right)-\varepsilon
$$

Adding up all such inequalities and collecting like terms, we find

$$
\mathbb{E}_{s_{0}, y}^{\psi^{\varepsilon}}\left[\int_{0}^{T-s} f^{\psi_{t}}\left(s_{0}+t, Y_{t}\right) e^{-\varphi_{t}} d t+g\left(Y_{T-s}\right) e^{-\varphi_{T-s}}\right]=J_{n_{0}}^{\psi^{\varepsilon}}\left(s_{0}, y\right) \geq w_{0}(y)-n \varepsilon .
$$

Then

$$
\sup _{\beta \in \Gamma(s)} \inf _{u \in \mathcal{M}(s)} J_{n}^{\psi}\left(s_{0}, y\right) \geq w_{0}(y)-n \varepsilon
$$

and letting $\varepsilon$ tend to zero we prove

$$
\overline{\Phi_{n}}\left(s_{0}, y\right) \geq w_{0}(y)=G_{s_{0}, s_{1}} G_{s_{1}, s_{2}} \ldots G_{s_{n-1}, s_{n}} g(y)
$$

Proof. (Thm 5.13) By lemma 5.12 we have

$$
\overline{\Phi_{n}}\left(s_{0}, y\right) \geq G_{s_{0}^{i}, s_{1}^{i}} G_{s_{1}^{i}, s_{2}^{i}} \ldots G_{s_{n(i)-1}^{i}, s_{n(i)}^{i}} g(y) .
$$

so that it remains to prove

$$
\overline{\Phi_{n}}\left(s_{0}, y\right) \leq \liminf _{i \rightarrow \infty} G_{s_{0}^{i}, s_{1}^{i}} G_{s_{1}^{i}, s_{2}^{i} \ldots} G_{s_{n(i)-1}^{i}, s_{n(i)}^{i}} g(y) .
$$

We give us step strategies $\left(u^{i}, v^{i}=\beta\left[u^{i}\right]\right)$ such that $J_{n}^{\psi^{i}}\left(s_{0}, y\right) \rightarrow \overline{\Phi_{n}}\left(s_{0}, y\right)$ as $i \rightarrow \infty$ and $u_{t}^{i}=u_{s_{j}^{i}-s_{0}}^{i}$ for $t \in\left[s_{j}^{i}-s_{0}, s_{j+1}^{i}-s_{0}\right)$. Then if we introduce $w_{n(i)}^{i}(y)=g(y), w_{j}^{i}(y)=$ 
$G_{s_{j}^{i}, s_{j+1}^{i}} w_{j+1}^{i}(y),(j=0, \ldots, n(i)-1)$, we get by the lemma 5.19 that

$$
\begin{array}{r}
\mathbb{E}_{s_{0}, y}^{\psi^{i}}\left[\int_{s_{j}^{i}-s_{0}}^{s_{j+1}^{i}-s_{0}} f^{\psi_{r}}\left(s_{0}+r, Y_{r}\right) e^{-\varphi_{r}} d r\right. \\
=\mathbb{E}_{s_{0}, y}^{\psi^{i}}\left[w_{j+1}^{i}\left(Y_{s_{j+1}^{i}-s_{0}}\right) e^{-\varphi_{s_{j}^{i}-s_{0}}} G_{s_{j}^{i} s_{j+1}^{i}-s_{0}}^{\psi_{j}^{i}-s_{0}} w_{j+1}^{i}\left(Y_{s_{j}^{i}-s_{0}}\right)\right] \\
\leq \mathbb{E}_{s_{0}, y}^{\psi^{i}}\left[e^{-\varphi_{s_{j}^{i}-s_{0}}} w_{j}^{i}\left(Y_{s_{j}^{i}-s_{0}}\right)\right] .
\end{array}
$$

Adding up such inequalities with respect to $j$ from $j=0$ to $j=n(i)-1$, and collecting like terms, we obtain:

$$
\mathbb{E}_{s_{0}, y}^{\psi^{i}}\left[\int_{0}^{T-s_{0}} f^{\psi_{r}}\left(s_{0}+r, Y_{r}\right) e^{-\varphi_{r}} d r+w_{n(i)}^{i}\left(Y_{s_{n}^{i}-s_{0}}\right) e^{-\varphi_{s_{n}^{i}-s_{0}}}\right] \leq w_{0}^{i}(y)
$$

that is $J_{n}\left(s_{0}, y, \psi^{i}\right) \leq w_{0}^{i}(x)$ and finally $\overline{\Phi_{n}}\left(s_{0}, y\right) \leq \liminf _{i \rightarrow \infty} w_{0}^{i}(y)$.

Proof. (Lemma 5.14) (a) Obviously

$$
\delta_{t}^{\psi, s, y}-K_{t}^{\psi, s, y}=-\mathbb{E}_{s, y}^{\psi}\left[g\left(Y_{T-s}\right) e^{-\varphi_{T-s}}+\int_{0}^{T-s} f^{\psi_{r}}\left(s+r, Y_{r}\right) e^{-\varphi_{r}} d r / \mathcal{F}_{t}\right]
$$

where the right side is a martingale. Hence $\delta_{t}^{\psi, s, y}$ is a supermartingale if $K_{t}^{\psi, s, y}$ is a supermartingale. The nonnegativity of $\delta_{t}^{\psi, s, y}$ is a consequence of the defintion of a supermartingale, $\delta_{t}^{\psi, s, y} \geq \mathbb{E}_{s, y}^{\psi}\left[\delta_{T-s} / \mathcal{F}_{t}\right]$ and $\delta_{T-s}^{\psi, s, y} \equiv 0$. Furthermore by the Prop 5.8, the function $\overline{\Phi_{n}}(s+t, y)$ is continuous with respect to $y$ and verifies $\left|\overline{\Phi_{n}}(s+t, y)\right| \leq N(1+|y|)^{m}$. Then by lemma 2.7 Krylov [1980] p144, $\lim _{n \rightarrow \infty} K_{t}^{\psi^{n}, s, y}=K_{t}^{\psi, s, y}, \forall t \in[0, T-s]$ if $\psi^{n} \rightarrow \psi$. We can choose step strategies $\psi^{n}$ so that we need prove $K_{t}^{\psi, s, x}$ is a supermartingale for step strategies only. It suffices to prove that $\mathbb{E}_{s, x}^{\psi}\left[K_{t_{2}} / \mathcal{F}_{t_{1}}\right] \leq K_{t_{1}}$ (a.s.) for $t_{2} \geq t_{1}$ if $u_{t}=u_{t_{1}} \in K$ on $\left[t_{1}, t_{2}\right.$ ) and $v_{t}=\beta\left[u_{t_{1}}\right] \in[0, n]$ for $t \in\left[t_{1}, t_{2}\right)$. We then have by the Lemma 5.19

$$
\begin{aligned}
\mathbb{E}_{s, y}^{\psi}\left[K_{t_{2}} / \mathbb{F}_{t_{1}}\right]=\underset{a . s .}{=} & \int_{0}^{t_{1}} f^{\psi_{r}}\left(s+r, Y_{r}^{\psi, s, y}\right) e^{-\varphi_{r}^{\psi, s, y}} d r \\
& +\mathbb{E}_{s, y}^{\psi}\left[\int_{t_{1}}^{t_{2}} f^{\psi_{t}}\left(s+t, Y_{t}\right) e^{-\varphi_{t}} d t+e^{-\varphi_{t_{2}}^{\psi, s, y}} \overline{\Phi_{n}}\left(s+t_{2}, Y_{t_{2}}\right) / \mathbb{F}_{t_{1}}\right] \\
= & \int_{0}^{t_{1}} f^{\psi_{r}}\left(s+r, Y_{r}\right) e^{-\varphi_{r}^{\psi, s, y}} d r \\
& +e^{-\varphi_{t_{1}}^{\psi, s, y}} G_{s+t_{1}, s+t_{2}}^{\psi_{t_{1}}} \overline{\Phi_{n}}\left(s+t_{2}, Y_{t_{1}}^{\psi, s, y}\right) .
\end{aligned}
$$

So that it remains to prove the assertion (b) of the lemma.

(b) Our objective is to prove that $G_{s, t} \overline{\Phi_{n}}(t, y) \leq \overline{\Phi_{n}}(s, y)\left(=\sup _{\beta} \inf _{u} J_{n}^{u, v=\beta[u]}(s, y)\right)$. We con- 
struct a sequence of subdivisions of $[t, T]$ whose diameter tends to zero:

$$
t=s_{0}=s_{0}^{i}<\ldots<s_{j}^{i}<\ldots<s_{n(i)}^{i}=T, \max _{j}\left(s_{j+1}^{i}-s_{j}^{i}\right) \underset{i \rightarrow+\infty}{\rightarrow} 0 .
$$

By lemma 5.12, Proposition 5.8 and the assumptions (A1)-(A5), we get for $\psi_{0}=\left(u_{0}, v_{0}\right) \in$ $K \times[0, n]$

$$
\begin{aligned}
N(1+|y|)^{m} \geq \overline{\Phi_{n}}(t, y) & \geq G_{s_{0}^{i}, s_{1}^{i}} G_{s_{1}^{i}, s_{2}^{i} \ldots G_{s_{n(i)-1}^{i}, s_{n(i)}^{i}} g(y)} \\
& \geq G_{s_{0}^{i}, s_{1}^{i}}^{\psi_{0}} G_{s_{1}^{i}, s_{2}^{i}}^{\psi_{0}} \ldots G_{s_{n(i)-1}^{i}, s_{n(i)}^{i}}^{\psi_{0}} g(y) \\
& =\mathbb{E}_{s, y}^{\psi_{0}}\left[+\int_{0}^{T-t} f^{\psi_{0}}\left(t+r, Y_{r}\right) e^{-\varphi_{r}} d r+g\left(Y_{T-t}\right) e^{-\varphi_{T-t}}\right] \\
& \geq-N(1+|y|)^{m}
\end{aligned}
$$

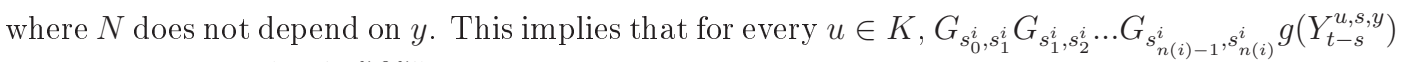
does not exceed $N\left(1+\left|Y_{t-s}^{u, s, y}\right|\right)^{m}$, the latter expression having a finite mathematical expectation. Then by $G_{s, t}^{\psi} G_{s_{0}^{i}, s_{1}^{i}} G_{s_{1}^{i}, s_{2}^{i} \ldots} \ldots G_{s_{n(i)-1}^{i}, s_{n(i)}^{i}} g(y)=\mathbb{E}_{s, y}^{\psi}\left[\int_{0}^{t-s} f^{\psi_{r}}\left(s+r, Y_{r}\right) e^{-\varphi_{r}} d r+w_{0}^{i}\left(Y_{t-s}\right) e^{-\varphi_{t-s}}\right]$, and the application of Lebesgue's theorem and Theorem 5.13, we deduce

$$
\begin{aligned}
\lim _{i \rightarrow \infty} G_{s, t}^{\psi} w_{0}^{i}(y) & =\mathbb{E}_{s, y}^{\psi}\left[\int_{0}^{t-s} f^{\psi_{r}}\left(s+r, Y_{r}\right) e^{-\varphi_{r}} d r+w_{0}^{i}\left(Y_{t-s}\right) e^{-\varphi_{t-s}}\right] \\
& =G_{s, t}^{\psi} \overline{\Phi_{n}}(t, y) .
\end{aligned}
$$

Finally by lemma 5.12, we have

$$
G_{s, t}^{\psi} \overline{\Phi_{n}}(t, y)=\lim _{i \rightarrow \infty} G_{s, t}^{\psi} G_{s_{0}^{i}, s_{1}^{i}} G_{s_{1}^{i}, s_{2}^{i} \ldots G_{s_{n(i)-1}^{i}, s_{n(i)}^{i}}} g(y) \leq \overline{\Phi_{n}}(s, y)
$$

Proof. (Lemma 5.16) First (b) is a consequence of (a). Indeed, by $(a)$ with $r_{t}^{u} \equiv 0$, we have

$$
\overline{\Phi_{n}}(s, y)=\inf _{u \in \mathcal{M}(s)} \mathbb{E}_{s, y}^{u}\left[\int_{0}^{\tau}\left[f^{u_{t}}+n\left(g-\overline{\Phi_{n}}\right)_{+}\right]\left(s+t, Y_{t}\right) e^{-\varphi_{t}} d t+\overline{\Phi_{n}}\left(s+\tau, Y_{\tau}\right) e^{-\varphi_{\tau}}\right]
$$

If $f_{n}^{u}:=f^{u}+n\left(g-\overline{\Phi_{n}}\right)_{+}$, since $f_{n}^{u} \geq f^{u}$ and that $\overline{\Phi_{n}} \geq g_{n}$, we have for every $\tau=\tau^{u}$,

$$
\begin{aligned}
\overline{\Phi_{n}}(s, y) & \geq \inf _{u \in \mathcal{M}(s)} \mathbb{E}_{s, y}^{u}\left[\int_{0}^{\tau} f^{u}\left(s+t, Y_{t}\right) e^{-\varphi_{t}} d t+g_{n}\left(s+\tau, Y_{\tau}\right) e^{-\varphi_{\tau}}\right] \\
& \geq \sup _{\tau \in \mathcal{T}_{s, T}} \inf _{u \in \mathcal{M}(s)} \mathbb{E}_{s, y}^{u}\left[\int_{0}^{\tau} f^{u}\left(s+t, Y_{t}\right) e^{-\varphi_{t}} d t+g_{n}\left(s+\tau, Y_{\tau}\right) e^{-\varphi_{\tau}}\right] .
\end{aligned}
$$

Furthermore if we define $\tau_{0} \equiv \inf \left\{t \geq 0, \overline{\Phi_{n}}\left(s+t, Y_{t}^{u}\right) \leq g\left(s+t, Y_{t}^{u}\right)\right\}$, for $t \in\left[0, \tau_{0}^{u}\right)$ we have 
$f_{n}^{u}\left(s+t, Y_{t}^{u}\right)=f^{u}\left(s+t, Y_{t}^{u}\right), \overline{\Phi_{n}}\left(s+t, Y_{t}^{u}\right)=g_{n}\left(s+t, Y_{t}^{u}\right)$ and in (2.86) we find

$$
\overline{\Phi_{n}}(s, y)=\inf _{u \in \mathcal{M}(s)} \mathbb{E}_{s, y}^{Y}\left[\int_{0}^{\tau_{0}^{u}} f^{u}\left(s+t, Y_{t}\right) e^{-\varphi_{t}} d t+g_{n}\left(s+\tau_{0}, Y_{\tau_{0}}^{u}\right) e^{-\varphi_{\tau_{0}}}\right] .
$$

Then combining (2.88) with (2.89), we deduce (b).

It remains to establish the assertion (a). In fact it is enough to prove it for $\tau^{u}=T-s$ and $r \equiv 0$.

Let $\psi=(u, \bar{v}) \in \mathcal{B}_{n}$ we introduce

$$
K_{t}=\overline{\Phi_{n}}\left(s+t, Y_{t}^{\psi, s, y}\right) e^{-\varphi_{t}^{\psi, s, y}}+\int_{0}^{t} f^{\psi_{p}}\left(s+p, Y_{p}^{\psi, s, y}\right) e^{-\varphi_{p}^{\psi, s, y}} d p
$$

and $\Phi_{t}=e^{\int_{0}^{t} \bar{v}_{p} d p}$. By the Lemma 5.14 the process $K_{t}$ is a supermartingale. Thus applying the lemma of Appendix 2 in Krylov [1980], the process $\rho_{t} \equiv K_{t} \phi_{t}-\int_{0}^{t} K_{s} d \phi_{s}$ is a supermartingale and

$$
\overline{\Phi_{n}}(s, y)=\mathbb{E} \rho_{0} \geq \mathbb{E} \rho_{T-s} \geq e^{n(T-s)}\left[\mathbb{E} K_{T-s}-\overline{\Phi_{n}}(s, y)\right]+\overline{\Phi_{n}}(s, y) .
$$

Using Fubini's theorem, we prove that

$$
\mathbb{E} \rho_{T-s}=\mathbb{E}_{s, y}^{u}\left[g\left(T, Y_{T-s}\right) e^{-\varphi_{T-s}}+\int_{0}^{T-s}\left[f^{u_{t}}+\bar{v}_{t}\left(g-\overline{\Phi_{n}}\right)\right]\left(s+t, Y_{t}\right) e^{-\varphi_{t}} d t\right]
$$

Further we note the upper bound of the last expression with respect to $\bar{v}$ is

$$
\mathbb{E}_{s, y}^{u}\left[g\left(T, Y_{T-s}\right) e^{-\varphi_{T-s}}+\int_{0}^{T-s}\left[f^{u_{t}}+n\left(g-\overline{\Phi_{n}}\right)_{+}\right]\left(s+t, Y_{t}\right) e^{-\varphi_{t}} d t\right]
$$

Thus taking the upper bound and the lower bound with respect to respectively $\bar{v}$ and $u$ in (2.90), we find

$$
\begin{aligned}
\overline{\Phi_{n}}(s, x) & \geq \inf _{u \in \mathcal{M}(s)} \mathbb{E}_{s, y}^{u}\left[\int_{0}^{T-s}\left[f^{u_{t}}+n\left(g-\overline{\Phi_{n}}\right)_{+}\right]\left(s+t, Y_{t}\right) e^{-\varphi_{t}} d t+g\left(T, Y_{T-s}\right) e^{-\varphi_{T-s}}\right] \\
& \geq e^{n(T-s)} \sup _{\bar{v}} \inf _{u \in \mathcal{M}(s)}\left(\mathbb{E}\left[K_{T-s}\right]-\overline{\Phi_{n}}(s, y)\right)+\overline{\Phi_{n}}(s, y) .
\end{aligned}
$$

Finally the expression of $K_{T-s}$ leads to $\sup _{\bar{v}} \inf _{u \in \mathcal{M}(s)} \mathbb{E}\left[K_{T-s}\right]-\overline{\Phi_{n}}(s, y)=0$ so that the expected equality follows.

Proof. (Proposition 5.11) (i) follows from the Proposition 5.8.

(ii) Since $\mathcal{B}_{n} \subset \mathcal{B}_{n+1}$, the sequence $\overline{\Phi_{n}}(s, y)$ increases. Moreover by Corollary $5.17 \overline{\Phi_{n}} \leq \Phi$ so that we define $\tilde{\Phi}(s, y)=\lim _{n \rightarrow \infty} \overline{\Phi_{n}}(s, y)$. By Corollary 5.17, $\tilde{\Phi}(s, y) \leq \Phi(s, y)$.

Recalling $\underline{\Phi_{n}}(s, y) \leq \overline{\Phi_{n}}(s, y)$ (see remark $5.10(\mathrm{i})$ ), let $\varepsilon>0$ and $\alpha^{\varepsilon} \in \Delta_{n}(s)$ such that

$$
\begin{aligned}
\overline{\Phi_{n}}(s, y) \geq \underline{\Phi_{n}}(s, y) & \left.\geq \sup _{v \in \mathcal{N}(s)} \mathbb{E}_{s, y}^{\alpha^{\varepsilon}, v}\left[\int_{0}^{T-s}\left[f^{\alpha_{t}^{\varepsilon}[v]}+v_{t} g\right)\right]\left(s+t, Y_{t}\right) e^{-\varphi t} d t+g\left(T, Y_{T-s}\right) e^{-\varphi_{T-s}}\right]-\varepsilon \\
& \left.\geq \mathbb{E}_{s, y}^{\alpha^{\varepsilon}, v}\left[\int_{0}^{T-s}\left[f^{\alpha_{t}^{\varepsilon}[v]}+v_{t} g\right)\right]\left(s+t, Y_{t}\right) e^{-\varphi_{t}} d t+g\left(T, Y_{T-s}\right) e^{-\varphi_{T-s}}\right]-\varepsilon, \forall v \in(\mathbb{\nabla}((2 s i)) .
\end{aligned}
$$


We take for $\tau \in \mathcal{T}_{s, T}, v_{t} \equiv n \chi_{\tau \leq t}$. Then, using Fubini's theorem we get

$$
\begin{aligned}
\overline{\Phi_{n}}(s, y) \geq & \mathbb{E}_{s, y}^{\alpha^{\varepsilon}}\left[\int_{\tau}^{T-s} n e^{-n(t-\tau)}\left[\int_{0}^{t} f^{\alpha_{p}^{\varepsilon}}\left(s+p, Y_{p}\right) e^{-\varphi_{p}} d p+g\left(s+t, Y_{t}\right) e^{-\varphi_{t}}\right] d t\right] \\
& +\mathbb{E}_{s, y}^{\alpha^{\varepsilon}}\left[e^{-n(T-s-\tau)}\left[\int_{0}^{T-s} f^{\alpha_{t}^{\varepsilon}}\left(s+t, Y_{t}\right) e^{-\varphi_{t}} d t+g\left(T, Y_{T-s}\right) e^{-\varphi_{T-s}}\right]\right]-\varepsilon \cdot(2.9
\end{aligned}
$$

We introduce

$$
\begin{aligned}
\eta^{\alpha^{\varepsilon}, s, y}(t) & =\int_{0}^{t} f^{\alpha_{p}^{\varepsilon}}\left(s+p, Y_{p}^{\alpha^{\varepsilon}, s, y}\right) e^{-\varphi_{p}^{\alpha^{\varepsilon}, s, y}} d p+g\left(s+t, Y_{t}^{\alpha^{\varepsilon}, s, y}\right) e^{-\varphi_{t}^{\alpha^{\varepsilon}, s, y}}, t \leq T-s \\
\eta^{\alpha^{\varepsilon}, s, y}(t) & =\eta(T-s), t>T-s .
\end{aligned}
$$

Furthermore we introduce a random variable $\xi$ which has an exponential distribution with a parameter equal to unity and which, in addition, does not depend on $\left\{\eta^{\alpha^{\varepsilon}, s, y}(t)\right\}$. We obtain $\overline{\Phi_{n}}(s, y) \geq \mathbb{E}_{s, y}^{\alpha^{\varepsilon}} \eta\left(\tau+\frac{1}{n} \xi\right)-\varepsilon$.

Therefore, by Lebesgue's theorem

$$
\begin{aligned}
& \tilde{\Phi}(s, y)+\varepsilon \geq \mathbb{E}_{s, y}^{\alpha^{\varepsilon}} \eta(\tau) \\
& \tilde{\Phi}(s, y)+\varepsilon \geq \sup _{\tau} \mathbb{E}_{s, y}^{\alpha^{\varepsilon}} \eta(\tau) \geq \inf _{\alpha} \sup _{\tau} \mathbb{E}_{s, y}^{\alpha^{\varepsilon}} \eta(\tau)
\end{aligned}
$$

Letting $\varepsilon$ tend to zero then $\tilde{\Phi}(s, y) \geq \Phi(s, y)$ and we conclude that $\tilde{\Phi}(s, y)=\Phi(s, y)$. The rest of the proof is a consequence of Dini's theorem and the property $\left|g_{n}(s, y)\right| \leq N(1+|y|)^{m}$ with the same constant $N$ for all $n, s, y$.

Proof. (Theorem 5.2)

Corollary 5.18, properties of supermartingales and $\Phi(s, y)=\rho_{0}$ imply

$$
\begin{aligned}
\Phi(s, y)=\mathbb{E}_{s, y}^{\psi}\left(\rho_{0}\right) \geq \mathbb{E}_{s, y}^{u}\left(\rho_{\tau}\right)= & \mathbb{E}_{s, y}^{u}\left[\int_{0}^{\tau}\left[f^{u_{t}}\left(s+t, Y_{t}\right)+\bar{v}_{t} \Phi\left(s+t, Y_{t}\right)\right] e^{-\varphi_{t}-\int_{0}^{t} \bar{v}_{p} d p} d t\right. \\
& \left.+\Phi\left(s+\tau, Y_{\tau}\right) e^{-\varphi_{\tau}-\int_{0}^{\tau} \bar{v}_{p} d p}\right]
\end{aligned}
$$

which proves inequality $(2.60)$.

Next let $\varepsilon>0 \tau_{u, s, y}^{\varepsilon}=\inf \left\{t \geq 0: \Phi\left(s+t, Y_{t}^{u, s, y}\right) \leq g\left(s+t, Y_{t}^{\alpha, s, y}\right)+\varepsilon\right\}$ and $\tau^{u} \leq \tau_{u, s, y}^{\varepsilon}$. According to DPP (see Prop 5.8) for each $n$,

$$
\begin{aligned}
\overline{\Phi_{n}}(s, y)= & \sup _{\beta \in \Delta_{n}(s)} \inf _{u \in \mathcal{M}(s)} E_{s, y}^{u, \beta}\left[\int_{0}^{\tau}\left(f^{u_{t}}+\beta[u]_{t} g\right)\left(s+t, Y_{t}\right) e^{-\varphi_{t}} d t\right. \\
& \left.+\overline{\Phi_{n}}\left(s+\tau, Y_{\tau}\right) e^{-\varphi_{\tau}}\right] \\
\leq & \sup _{\beta \in \Delta(s)} \inf _{u \in \mathcal{M}(s)} E_{s, y}^{u, \beta}\left[\int_{0}^{\tau}\left(f^{u_{t}}+\beta[u]_{t} g\right)\left(s+t, Y_{t}\right) e^{-\varphi_{t}} d t\right. \\
& \left.+\Phi\left(s+\tau, Y_{\tau}\right) e^{-\varphi_{\tau}}\right] .
\end{aligned}
$$


Taking the limit as $n \rightarrow \infty$ and using the fact that $\overline{\Phi_{n}} \nearrow \Phi$ we have

$$
\Phi(s, y) \leq \sup _{\beta \in \Delta(s)} \inf _{u \in \mathcal{M}(s)} E_{s, y}^{u, \beta}\left[\int_{0}^{\tau}\left(f^{u_{t}}+\beta[u]_{t} g\right)\left(s+t, Y_{t}\right) e^{-\varphi_{t}} d t+\Phi\left(s+\tau, Y_{\tau}\right) e^{-\varphi_{\tau}}\right]
$$

Now by (2.94) and the inequality $\Phi \geq g$ we get

$$
\Phi(s, y) \geq E_{s, y}^{u, \beta}\left[\int_{0}^{\tau}\left(f^{u_{t}}+\beta[u]_{t} g\right)\left(s+t, Y_{t}\right) e^{-\varphi_{t}} d t+\Phi\left(s+\tau, Y_{\tau}\right) e^{-\varphi_{\tau}}\right] .
$$

so that combining (2.95) and (2.96) we find

$$
\Phi(s, y)=\sup _{\beta \in \Delta(s)} \inf _{u \in \mathcal{M}(s)} E_{s, y}^{u, \beta}\left[\int_{0}^{\tau}\left(f^{u_{t}}+\beta[u]_{t} g\right)\left(s+t, Y_{t}\right) e^{-\varphi_{t}} d t+\Phi\left(s+\tau, Y_{\tau}\right) e^{-\varphi_{\tau}}\right]
$$

Further, we take a sequence $u^{i} \in \mathcal{M}(s)$ and $\beta^{i}=\beta\left[u^{i}\right] \in \Delta(s)$ such that

$$
\Phi(s, y)=\lim _{i \rightarrow \infty} E_{s, y}^{u^{i}}\left[\int_{0}^{\tau}\left(f^{u_{t}^{i}}+\beta\left[u^{i}\right]_{t} g\right)\left(s+t, Y_{t}\right) e^{-\varphi_{t}^{u^{i}, \beta}} d t+\Phi\left(s+\tau, Y_{\tau}\right) e^{-\varphi_{\tau}^{u^{i}}-\int_{0}^{\tau} \beta\left[u^{i}\right]_{p} d p}\right] .
$$

From $g\left(s+t, Y_{t}^{u, s, y}\right)<\Phi\left(s+t, Y_{t}^{u, s, y}\right)-\varepsilon$ for $t<\tau_{\varepsilon}^{u, s, y}$, (2.94) and (2.98), we find

$$
\varepsilon \lim _{i \rightarrow \infty} E_{s, y}^{u^{i}}\left[\int_{0}^{\tau} \beta\left[u^{i}\right]_{t} e^{-\varphi_{t}-\int_{0}^{\tau} \beta\left[u^{i}\right]_{p} d p} d t\right]=0
$$

By lemma 4.2 in Krylov [1980] p 153, (2.99) and (2.98) we deduce

$$
\Phi(s, y)=\lim _{i \rightarrow \infty} E_{s, y}^{u^{i}}\left[\int_{0}^{\tau} f^{u_{t}^{i}}\left(s+t, Y_{t}\right) e^{-\varphi_{t}} d t+\Phi\left(s+\tau, Y_{\tau}\right) e^{-\varphi_{\tau}}\right] .
$$

On the other hand by Corollary 5.18 , the process

$$
K_{t}^{u, s, y}=\Phi\left(s+t, Y_{t}^{u, s, y}\right) e^{-\varphi_{t}^{u, s, y}}+E_{s, y}^{u^{i}}\left[\int_{0}^{t} f^{u_{p}^{i}}\left(s+p, Y_{p}\right) e^{-\varphi_{p}} d p\right]
$$

is a continuous supermartingale. Therefore according with lemma given in appendix 2 in Krylov [1980], $K_{t}^{u, s, y}-\rho_{t}^{Y, \beta[u], s, y}$ is a supermartingale for each $\psi=(u, \beta[u])$. In particular, $E_{s, y}^{u} K_{\tau} \leq E_{s, y}^{\psi} \rho_{\tau}$ which together with (2.100) and (2.94) yields

$$
\begin{aligned}
\Phi(s, y)=\lim _{i \rightarrow \infty} E_{s, y}^{u^{i}} K_{\tau} \leq & \lim _{i \rightarrow \infty} E_{s, y}^{u^{i}, \beta\left[u^{i}\right]} \rho_{\tau} \\
& =\lim _{i \rightarrow \infty} E_{s, y}^{u^{i}}\left[\Phi\left(s+\tau, Y_{\tau}\right) e^{-\varphi_{\tau}-\int_{0}^{\tau} \beta\left[u^{i}\right]_{p} d p}\right. \\
& \left.+\int_{0}^{\tau}\left(f^{u_{t}^{i}}+\beta\left[u^{i}\right]_{t} \Phi\right)\left(s+t, Y_{t}\right) e^{-\varphi_{t}-\int_{0}^{t} \beta\left[u^{i}\right]_{p} d p} d t\right] .
\end{aligned}
$$


Thus

$$
\begin{aligned}
\Phi(s, y) \leq & E_{s, y}^{u}\left[\int_{0}^{\tau}\left(f^{u_{t}}+\beta[u]_{t} \Phi\right)\left(s+t, Y_{t}\right) e^{-\varphi_{t}-\int_{0}^{t} \beta[u]_{p} d p} d t\right. \\
& \left.+\Phi\left(s+\tau, Y_{\tau}\right) e^{-\varphi_{\tau}-\int_{0}^{\tau} \beta[u]_{p} d p}\right] \\
\leq & \Phi(s, y) .
\end{aligned}
$$

The required equality is then proved. 


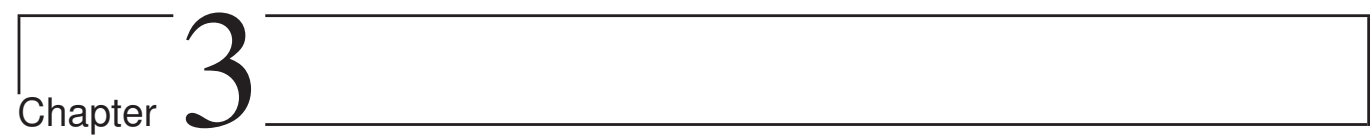

Maximum Principles for Jump Diffusion

Processes with Infinite Horizon

With Bernt Øksendal and Frank Proske.

Published in Automatica. 


\subsection{Introduction}

In this paper we consider a control problem for a performance functional

$$
J(u)=E\left[\int_{0}^{\infty} f(t, X(t), u(t), \omega) d t\right],
$$

where $X(t)$ is a controlled jump diffusion and $u(t)$ is the control process. We allow for the case where the controller only has access to partial information. Thus, we have an infinite horizon problem with partial information. Infinite-horizon optimal control problems arise in many fields of economics, in particular in models of economic growth. Note that because of the general nature of the partial information filtration $\mathcal{E}_{t}$, we cannot use dynamic programming and Hamilton-Jacobi-Bellman (HJB) equations to solve the optimization problem. Thus our problem is different from partial observation control problems.

Stochastic differential equations with jumps are important, because they can provide us with more realistic models. For example, jump processes are natural in finance, because stock prices often exhibit jump type behavior. Moreover, financial markets with stock prices modeled by jump processes provide a rich family of incomplete financial models. For more information about financial markets with jump processes see e.g. Cont and Tankov [2004] and the references therein.

Infinite horizon models are studied in connection with several disciplines, and extensively in economics. Important examples are the perpetual American option and sustainability issues in optimal resource extraction. Again it is important to be able to deal with jump models, because they often are more realistic. When it comes to stochastic control, the possibly infinite horizon does not cause any problems when using dynamic programming. However, dynamic programming can only be used for Markovian models. In non-Markovian models the alternative stochastic control method called the maximum principle can be used in stead. However, the drawback here has been that traditionally it requires a finite terminal time for the associated backward stochastic differential equation (BSDE). Thus, the problem has been: If the terminal time $\mathrm{T}$ is infinite, what is the correct substitute for the terminal value of the solution of the BSDE?

In the deterministic case the maximum principle by Pontryagin (1962) has been extended to infinite-horizon problems, but transversality conditions have not been given in general. The 'natural' transversality condition in the infinite case would be a zero limit condition, meaning in the economic sense that one more unit of good at the limit gives no additional value. But this property is not necessarily verified. In fact Halkin [1974] provides a counterexample for a 'natural' extension of the finite-horizon transversality conditions. Thus some care is needed in the infinite horizon case, and we emphasize that the maximum principle for infinite horizon control is not a straightforward extension of the finite horizon case. We explain this in more detail at the end of section 2. To construct an infinite horizon maximum principle a solution of an infinite horizon backward stochastic differential equation (BSDE) is required. While Pardoux in Pardoux [1999] tackles these kind of equations, they are without jumps. As far as the authors know there have been no papers on infinite horizon BSDE's with jumps. Existence of such equations will most likely be useful in a lot more areas than optimal control.

There are earlier papers on infinite horizon problems. E.g. in Maslowski and Veverka [2011] it is stated a 'natural' extension to infinite horizon discounted control problems. Our paper on the other hand is (to our knowledge) the first which requires a limit inequality on the terminal condition. This seems to be more in line with deterministic theory. See the discussion at the end of Section 2. This type of limit inequality condition is not caused by the presence of jumps, but in view of the interest in jump models as explained above, it is important to give a proof of the limit inequality condition that works also if there are jumps. But we emphasize that also in the no-jump case our condition is new. 
We refer to Sulem and Øksendal [2007] for more information about stochastic control in jump diffusion markets, to Peng and Shi [2000] for a background on backward stochastic differential equations and Seierstad et al. [2006] for a general introduction to infinite-horizon control problems in a deterministic environment.

In this paper we prove several maximum principles for an infinite horizon optimal control problem with partial information. The paper is structured as follows: In Section 4 we prove a maximum principle version of sufficient type (a verification theorem). In Section 5 we give some examples, before we prove a (weak) version of a necessary type of the maximum principle in Section 6.

In a forthcoming paper Agram et al. [2013], the case of infinite horizon for delay equations is treated.

\subsection{Preliminaries}

Let $B(t)=B(t, \omega)=\left(B_{1}(t, \omega), \ldots, B_{n}(t, \omega)\right), t \geq 0, \omega \in \Omega$ and $\tilde{N}(d z, d t)=N(d z, d t)-$ $\nu(d z) d t=\left(\tilde{N}_{1}(d z, d t), \ldots, \tilde{N}_{n}(d z, d t)\right)$ be an n-dimensional Brownian motion and $\mathrm{m}$ independent compensated Poisson random measures, respectively, on a filtered probability space, $\left(\Omega, \mathcal{F},\left\{\mathcal{F}_{t}\right\}_{t \geq 0}, P\right)$, satisfying the usual conditions. Let $X(t)=X^{u}(t)$ be a controlled jump diffusion, described by the stochastic differential equation

$$
\begin{aligned}
d X(t) & =b(t, X(t), u(t), \omega) d t+\sigma(t, X(t), u(t), \omega) d B(t) \\
& +\int_{\mathbb{R}_{0}^{n}} \theta(t, X(t), u(t), z, \omega) \tilde{N}(d z, d t) ; 0 \leq t<\infty \\
X(0) & =x \in \mathbb{R}^{n}
\end{aligned}
$$

where $\mathbb{R}_{0}=\mathbb{R} \backslash\{0\}, b:[0, \infty] \times \mathbb{R}^{n} \times U \times \Omega \rightarrow \mathbb{R}^{n}$ is adapted, $\sigma:[0, \infty] \times \mathbb{R}^{n} \times U \times \Omega \rightarrow \mathbb{R}^{n \times n}$ is adapted with respect to $\left\{\mathcal{F}_{t}\right\}_{t \geq 0}$ and $\theta:[0, \infty] \times \mathbb{R}^{n} \times U \times \Omega \rightarrow \mathbb{R}^{n \times n}$ is predictable with respect to $\left\{\mathcal{F}_{t}\right\}_{t \geq 0}$ (see Rogers and Williams [2000b]). See e.g. Baghery and Øksendal [2007], Sulem and Øksendal [2007] for notation and more information. Let

$$
\mathcal{E}_{t} \subset \mathcal{F}_{t},
$$

be a given subfiltration, representing the information available to the controller at time $t ; t \geq 0$. The process $u(t)$ is our control, assumed to be $\left\{\varepsilon_{t}\right\}_{t>0}$ predictable and with values in a convex set $U \subset \mathbb{R}^{n}$. Let $\mathcal{A}_{\mathcal{E}}$ be our family of $\mathcal{E}_{t}$-predictable controls. Let $\mathcal{R}$ denote the set of functions $r:[0, \infty] \times \mathbb{R}_{0}^{n} \rightarrow \mathbb{R}^{n \times n}$ such that

$$
\int_{\mathbb{R}_{0}^{n}}\left|\theta_{i, j}(t, x, u, z) r_{i, j}(t, z)\right| \nu_{j}(d z)<\infty \text { for all } i, j, t, x .
$$

Let $f:[0, \infty] \times \mathbb{R}^{n} \times U \times \Omega \rightarrow \mathbb{R}^{n}$ be adapted with respect to $\left\{\mathcal{F}_{t}\right\}_{t \geq 0}$ and assume that

$$
E\left[\int_{0}^{\infty}|f(t, X(t), u(t), \omega)| d t\right]<\infty \text { for all } u \in \mathcal{A}_{\mathcal{E}} .
$$


Then we define

$$
J(u)=E\left[\int_{0}^{\infty} f(t, X(t), u(t), \omega) d t\right]
$$

to be our performance functional. We study the problem to find $\hat{u} \in \mathcal{A}_{\mathcal{E}}$ such that

$$
J(\hat{u})=\sup _{u \in \mathcal{A}_{\varepsilon}} J(u)
$$

Let us define the Hamiltonian $H:[0, T] \times \mathbb{R}^{n} \times U \times \mathbb{R}^{n} \times \mathbb{R}^{n \times n} \times \mathcal{R} \rightarrow \mathbb{R}$, by

$$
\begin{aligned}
H(t, x, u, p, q, r) & =f(t, x, u, \omega)+b^{T}(t, x, u, \omega) p+\operatorname{tr}\left(\sigma^{T}(t, x, u, \omega) q\right) \\
& +\sum_{i, j=1}^{n} \int_{\mathbb{R}_{0}^{n}} \theta_{i, j}(t, x, u, z, \omega) r_{i, j}(t, z) \nu_{j}(d z) .
\end{aligned}
$$

For notational convenience we will in the rest of the paper suppress $\omega$ from the notation. The adjoint equation in the unknown $\mathcal{F}_{t}$-predictable processes $(p(t), q(t), r(t, z))$ is the following

$$
\begin{aligned}
d p(t) & =-\nabla_{x} H(t, X(t), u(t), p(t), q(t), r(t, \cdot)) d t+q(t) d B(t) \\
& +\int_{\mathbb{R}_{0}^{n}} r(t, z) \tilde{N}(d z, d t), t \geq 0 .
\end{aligned}
$$

In the finite horizon case (replacing $\infty$ by a finite terminal time $T$ in $J(u)$ above) the adjoint variable $p(t)$ would have the specified terminal value

$$
p(T)=0
$$

In the infinite horizon case it is natural to guess that the corresponding terminal condition would be

$$
\lim _{t \rightarrow \infty} p(t)=0
$$

However, this turns out to be incorrect: The terminal condition must be replaced by a limit inequality. See Theorem 4.1 and Theorem 6.1. This illustrates that the infinite horizon case requires new techniques, and it cannot be deduced from the finite horizon case.

\subsection{Existence and Uniqueness}

In this section we prove a result about existence and uniqueness of the solution $(Y(t), Z(t), K(t, \zeta))$ of infinite horizon BSDEs of the form;

$$
\begin{aligned}
d Y(t) & =-g(t, Y(t), Z(t), K(t, \cdot)) d t+Z(t) d B(t) \\
& +\int_{\mathbb{R}_{0}^{n}} K(t, \zeta) \tilde{N}(d \zeta, d t) ; 0 \leq t \leq \tau, \\
\lim _{t \rightarrow \tau} Y(t) & =\xi(\tau) \mathbf{1}_{[0, \infty)}(\tau),
\end{aligned}
$$


where $\tau \leq \infty$ is a given $\mathcal{F}_{t^{-}}$stopping time, possibly infinite. Our result is an extension to jumps of Theorem 4.1 in Pardoux [1999], Theorem 4 in Peng and Shi [2000] and Theorem 3.1 in Yin [2008]. It is also an extension to infinite horizon of Lemma 2.1 in Li and Peng [2009]. See also Li and Tang [1994], Royer [2006], Barles et al. [2009] and Situ [2002]. The proof in the jump case is not much different from the non-jump case; but we include it for completeness. We assume the following:

1. The function $g: \Omega \times \mathbb{R}_{+} \times \mathbb{R}^{k} \times \mathbb{R}^{k \times d} \times \mathcal{R} \rightarrow \mathbb{R}^{k}$ is such that there exist real numbers $\mu, \lambda, K_{1}$ and $K_{2}$, such that $K_{1}, K_{2}>0$ and

$$
\lambda>2 \mu+K_{1}^{2}+K_{2}^{2}
$$

We assume that the function $g$ satisfies the following requirements:

(a) $g(\cdot, y, z, k)$ is progressively measurable for all $y, z, k$, and

$$
\begin{aligned}
& \left|g(t, y, z, k(\cdot))-g\left(t, y, z^{\prime}, k^{\prime}(\cdot)\right)\right| \leq K_{1}\left\|z-z^{\prime}\right\| \\
& +K_{2}\left\|k(\cdot)-k^{\prime}(\cdot)\right\|_{R},
\end{aligned}
$$

where

$$
\|k(\cdot)\|_{R}^{2}=\int_{\mathbb{R}_{0}^{n}} k^{2}(\zeta) \nu(d \zeta)
$$

and $\|z\|=\left[\operatorname{Tr}\left(z z^{*}\right)\right]^{\frac{1}{2}}$.

(b)

$$
\left\langle y-y^{\prime}, g(t, y, z, k)-g\left(t, y^{\prime}, z, k\right)\right\rangle \leq \mu\left|y-y^{\prime}\right|^{2}
$$

for all $y, y^{\prime}, z, k$ P-a.s.

(c)

$$
E \int_{0}^{\tau} e^{\lambda t}|g(t, 0,0,0)|^{2} d t<\infty
$$

(d) Finally we require that

$$
y \mapsto g(t, y, z, k)
$$

is continuous for all $t, z, k$ P-a.s.

2. We have a final condition on $\xi$, which is $\mathcal{F}_{\tau}$-measurable such that $E\left(e^{\lambda \tau}|\xi|^{2}\right)<\infty$ and

$$
E \int_{0}^{\tau} e^{\lambda t}\left|g\left(t, \xi_{t}, \eta_{t}, \psi_{t}\right)\right|^{2} d t<\infty
$$

where $\xi_{t}=E\left(\xi \mid \mathcal{F}_{t}\right)$ and $\eta, \psi$ are $\left\{\mathcal{F}_{t}\right\}_{t \geq 0}$-adapted and $\left\{\mathcal{F}_{t}\right\}_{t \geq 0}$-predictable, respectively, s.t.

$$
\xi=E \xi+\int_{0}^{t} \eta(s) d B_{s}+\int_{0}^{t} \int_{\mathbb{R}_{0}^{n}} \psi(s, \zeta) \tilde{N}(d \zeta, d s) .
$$


A solution of the BSDE (3.5)-(3.6), is a triple $\left(Y_{t}, Z_{t}, K_{t}\right)$ of progressively measurable processes with values in $\mathbb{R}^{k} \times \mathbb{R}^{k \times d} \times \mathcal{R}$ s.t. $Z_{t}, K_{t}=0$ when $t>\tau$,

1. $E\left[\sup _{t \geq 0} e^{\lambda t}\left|Y_{t}\right|^{2}+\int_{0}^{\tau} e^{\lambda s}\left|Z_{s}\right|^{2} d s+\int_{0}^{\tau} \int_{\mathbb{R}_{0}^{n}} e^{\lambda s} K^{2}(s, \zeta) \nu(d \zeta) d s\right]<\infty$,

2. $Y_{t}=Y_{T \wedge \tau}+\int_{t \wedge \tau}^{T \wedge \tau} g_{s} d s-\int_{t \wedge \tau}^{T \wedge \tau} Z_{s} d B_{s}-\int_{t \wedge \tau}^{T \wedge \tau} \int_{\mathbb{R}_{0}^{n}} K(s, \zeta) \tilde{N}(d \zeta, d s)$ for all deterministic $T<\infty$ and

3. $Y_{t}=\xi$ on the set $\{t \geq \tau\}$.

Remark 3.1 (Infinite Horizon). This incorporates the case where $\tau(\omega)=\infty$ on some set $A$ with $P(A)>0$, possibly $P(A)=1$.

Theorem 3.2 (Existence and uniqueness). Under the above conditions there exists a unique solution $\left(Y_{t}, Z_{t}, K_{t}\right)$ of the BSDE (3.5)-(3.6), which satisfies the condition;

$$
\begin{aligned}
& E\left[\sup _{0 \leq t \leq \tau} e^{\lambda t}\left|Y_{t}\right|^{2}+\int_{0}^{\tau} e^{\lambda s}\left(\left|Y_{s}\right|^{2}+\left\|Z_{s}\right\|^{2}\right) d s+\int_{0}^{\tau} e^{\lambda s} \int_{\mathbb{R}_{0}^{n}} K^{2}(s, \zeta) \nu(d \zeta) d s\right] \\
& \leq c E\left[e^{\lambda \tau}|\xi|^{2}+\int_{0}^{\tau} e^{\lambda s}|g(s, 0,0,0)|^{2} d s\right]
\end{aligned}
$$

for some positive number $c$.

Proof. First, let us show uniqueness. Let $(Y, Z, K)$ and $\left(Y^{\prime}, Z^{\prime}, K^{\prime}\right)$ be two solutions satisfying (3.14) and let $(\bar{Y}, \bar{Z}, \bar{K})=\left(Y-Y^{\prime}, Z-Z^{\prime}, K-K^{\prime}\right)$. From It $\bar{o}$ 's Lemma we have that

$$
\begin{aligned}
& e^{\lambda t \wedge \tau}\left|\bar{Y}_{t \wedge \tau}\right|^{2}+\int_{t \wedge \tau}^{T \wedge \tau}\left[e^{\lambda s}\left(\lambda\left|\bar{Y}_{s}\right|^{2}+\left\|\bar{Z}_{s}\right\|^{2}\right)+e^{\lambda s} \int_{\mathbb{R}_{0}^{n}} \bar{K}^{2}(s, \zeta) \nu(d \zeta)\right] d s \\
& \leq e^{\lambda s}\left|\bar{Y}_{T}\right|^{2}+2 \int_{t \wedge \tau}^{T \wedge \tau}\left[e^{\lambda s}\left(\mu\left|\bar{Y}_{s}\right|^{2}+K_{1}\left|\bar{Y}_{s}\right| \times\left\|\bar{Z}_{s}\right\|\right)\right. \\
& \left.+K_{2}\left|\bar{Y}_{s}\right| e^{\lambda s}\left(\int_{\mathbb{R}_{0}^{n}} \bar{K}^{2}(s, \zeta) \nu(d \zeta)\right)^{\frac{1}{2}}\right] d s-2 \int_{t \wedge \tau}^{T \wedge \tau} e^{\lambda s}\left\langle\bar{Y}_{s}, \bar{Z}_{s} d B_{s}\right\rangle \\
& -\int_{t \wedge \tau}^{T \wedge \tau} e^{\lambda s} \int_{\mathbb{R}_{0}^{n}}\left[\bar{K}^{2}(s, \zeta)+2 \bar{K}(s, \zeta) \bar{Y}(s)\right] \tilde{N}(d \zeta, d s) .
\end{aligned}
$$

Combining the above with the fact that $2 a b \leq a^{2}+b^{2}$ we deduce since $\lambda>2 \mu+K_{1}^{2}+K_{2}^{2}$, that for $t<T$

$$
E\left[e^{\lambda t \wedge \tau}\left|\bar{Y}_{t \wedge \tau}\right|^{2}\right] \leq E\left[e^{\lambda T \wedge \tau}\left|\bar{Y}_{T}\right|^{2}\right]
$$

the same holds with $\lambda$ replaced by $\lambda^{\prime}$, with $\lambda>\lambda^{\prime}>2 \mu+K_{1}^{2}+K_{2}^{2}$

$$
E\left[e^{\lambda t \wedge \tau}\left|\bar{Y}_{t \wedge \tau}\right|^{2}\right] \leq e^{\left(\lambda-\lambda^{\prime}\right) T} E\left[e^{\lambda T \wedge \tau}\left|\bar{Y}_{T}\right|^{2} \mathbf{1}_{\{T<\tau\}}\right]
$$

Condition (3.14) implies that the second factor on the right hand side remains bounded as $T \rightarrow \infty$, while the first factor tends to 0 . This proves uniqueness. 
Proof of existence. For each $n \in \mathrm{N}$ we construct a solution $\left(Y_{t}^{n}, Z_{t}^{n}, K_{t}^{n}\right)$ of the BSDE

$$
\begin{aligned}
Y_{t}^{n} & =\xi+\int_{t \wedge \tau}^{n \wedge \tau} g\left(s, Y_{s}^{n}, Z_{s}^{n}, K_{s}^{n}\right) d s-\int_{t \wedge \tau}^{\tau} Z_{s}^{n} d B_{s} \\
& -\int_{t \wedge \tau}^{\tau} \int_{\mathbb{R}_{0}^{n}} K^{n}(s, \zeta) \tilde{N}(d \zeta, d s)
\end{aligned}
$$

by letting $\left\{\left(Y_{t}^{n}, Z_{t}^{n}, K_{t}^{n}\right) ; 0 \leq t \leq n\right\}$ be defined as a solution of the following BSDE:

$$
\begin{aligned}
Y_{t}^{n} & =E\left[\xi \mid \mathcal{F}_{n}\right]+\int_{t}^{n} \mathbf{1}_{[0, \tau]}(s) g\left(s, Y_{s}^{n}, Z_{s}^{n}, K_{s}^{n}\right) d s \\
& -\int_{t}^{n} Z_{s}^{n} d B_{s}-\int_{t}^{n} \int_{\mathbb{R}_{0}^{n}} K^{n}(s, \zeta) \tilde{N}(d \zeta, d s)
\end{aligned}
$$

for $0 \leq t \leq n$ and $\left\{\left(Y_{t}^{n}, Z_{t}^{n}, K_{t}^{n}\right) ; t \geq n\right\}$ defined by

$$
\begin{aligned}
& Y_{t}^{n}=\xi_{t}, \\
& Z_{t}^{n}=\eta_{t},
\end{aligned}
$$

and

$$
K_{t}^{n}=\psi_{t},
$$

for $t>n$. Next, we find some a priori estimates for the sequence $\left(Y^{n}, Z^{n}, K^{n}\right)$. For any $\epsilon>0$, $\rho<1$ and $\alpha$ we have for all $t \geq 0, y \in \mathbb{R}^{k}, z \in \mathbb{R}^{k \times d}, k \in \mathcal{R}$ with $c=\frac{1}{\epsilon}$,

$$
\begin{aligned}
& 2\langle y, g(t, y, z, k)\rangle=2\langle y, g(t, y, z, k)-g(t, 0, z, k)\rangle \\
& +2\langle y, g(t, 0, z, k)-g(t, 0,0,0)\rangle+2\langle y, g(t, 0,0,0)\rangle \\
& \leq\left(2 \mu+\frac{1}{\rho} K_{1}^{2}+\frac{1}{\alpha} K_{2}^{2}+\epsilon\right)|y|^{2}+\rho\|z\|^{2} \\
& +\alpha \int_{\mathbb{R}_{0}^{n}} k^{2}(\zeta) \nu(d \zeta)+c|g(t, 0,0,0)|^{2} .
\end{aligned}
$$

From Itō's Lemma we have

$$
\begin{aligned}
& e^{\lambda t \wedge \tau}\left|Y_{t \wedge \tau}^{n}\right|^{2}+\int_{t \wedge \tau}^{\tau}\left[e^{\lambda s}\left(\bar{\lambda}\left|Y_{s}^{n}\right|^{2}+\bar{\rho}\left\|Z_{s}^{n}\right\|^{2}\right)\right. \\
& \left.+\bar{\alpha} \int_{t \wedge \tau}^{\tau} e^{\lambda s} \int_{\mathbb{R}_{0}^{n}}\left(K^{n}\right)^{2}(s, \zeta) \nu(d \zeta)\right] d s \\
& \leq e^{\lambda s}|\eta|^{2}+c \int_{t \wedge \tau}^{\tau} e^{\lambda s}|g(s, 0,0,0)|^{2} d s \\
& -2 \int_{t \wedge \tau}^{\tau} e^{\lambda s}<Y_{s}^{n}, Z_{s}^{n} d B_{s}> \\
& -\int_{t \wedge \tau}^{\tau} e^{\lambda s} \int_{\mathbb{R}_{0}^{n}}\left[\left(K^{n}\right)^{2}(s, \zeta)+2 K^{n}(s, \zeta) Y^{n}(s)\right] \tilde{N}(d \zeta, d s),
\end{aligned}
$$


with $\bar{\lambda}=\lambda-2 \mu-\frac{1}{\rho} K_{1}^{2}-\frac{1}{\alpha} K_{2}^{2}-\epsilon>0, \bar{\rho}=1-\rho>0$ and $\bar{\alpha}=1-\alpha$. From this and the martingale inequality it follows that

$$
\begin{aligned}
& E\left[\sup _{t \geq s} e^{\lambda t \wedge \tau}\left|Y_{t \wedge \tau}^{n}\right|^{2}+\int_{s \wedge \tau}^{\tau}\left[e^{\lambda r}\left(\left|Y_{r}^{n}\right|^{2}+\left\|Z_{r}^{n}\right\|^{2}\right)\right.\right. \\
& \left.\left.+e^{\lambda r} \int_{\mathbb{R}_{0}^{n}}\left(K^{n}\right)^{2}(r, \zeta) \nu(d \zeta)\right] d r\right] \\
& \leq D E\left[e^{\lambda \tau}|\xi|^{2}+\int_{s \wedge \tau}^{\tau} e^{\lambda r}|g(r, 0,0,0)|^{2} d r\right] .
\end{aligned}
$$

Let $m>n$ and define $\Delta Y_{t}:=Y_{t}^{m}-Y_{t}^{n}, \Delta Z_{t}:=Z_{t}^{m}-Z_{t}^{n}$ and $\Delta K_{t}:=K_{t}^{m}-K_{t}^{n}$, so that for $n \leq t \leq m$,

$$
\begin{aligned}
\Delta Y_{t} & =\int_{t \wedge \tau}^{m \wedge \tau} g\left(s, Y_{s}^{m}, Z_{s}^{m}, K_{s}^{m}\right) d s-\int_{t \wedge \tau}^{m \wedge \tau} \Delta Z_{s} d B_{s} \\
& -\int_{t \wedge \tau}^{m \wedge \tau} \int_{\mathbb{R}_{0}^{n}} \Delta K(s, \zeta) \tilde{N}(d \zeta, d s) .
\end{aligned}
$$

It then follows that

$$
\begin{aligned}
& e^{\lambda t \wedge \tau}\left|\Delta Y_{t \wedge \tau}\right|^{2}+\int_{t \wedge \tau}^{m \wedge \tau}\left\{e^{\lambda s}\left(\lambda\left|\Delta Y_{s}\right|^{2}+\left\|\Delta Z_{s}\right\|^{2}\right)+e^{\lambda s} \int_{\mathbb{R}_{0}^{n}}(\Delta K)^{2}(s, \zeta) \nu(d \zeta)\right\} d s \\
& =\int_{t \wedge \tau}^{m \wedge \tau} e^{\lambda s}\left\langle\Delta Y_{s}, g\left(s, Y_{s}^{m}, Z_{s}^{m}, K_{s}^{m}\right)\right\rangle d s-2 \int_{t \wedge \tau}^{m \wedge \tau} e^{\lambda s}\left\langle\Delta Y_{s}, \Delta Z_{s} d B_{s}\right\rangle \\
& -\int_{t \wedge \tau}^{m \wedge \tau} e^{\lambda s} \int_{\mathbb{R}_{0}^{n}}\left[(\Delta K)^{2}(s, \zeta)+2 \Delta K(s, \zeta) \Delta Y(s)\right] \tilde{N}(d \zeta, d s) \\
& \leq 2|\eta|^{2} c \int_{t \wedge \tau}^{m \wedge \tau} e^{\lambda s}|g(s, 0,0,0)|^{2} d s-2 \int_{t \wedge \tau}^{m \wedge \tau} e^{\lambda s}\left\langle\Delta Y_{s}, \Delta Z_{s} d B_{s}\right\rangle \\
& -\int_{t \wedge \tau}^{m \wedge \tau} e^{\lambda s} \int_{\mathbb{R}_{0}^{n}}\left[(\Delta K)^{2}(s, \zeta)+2 \Delta K(s, \zeta) \Delta Y(s)\right] \tilde{N}(d \zeta, d s) .
\end{aligned}
$$

From the same arguments as above

$$
\begin{aligned}
& E\left[\sup _{n \leq t \leq m} e^{\lambda t \wedge \tau}\left|\Delta Y_{t \wedge \tau}\right|^{2}+\int_{n \wedge \tau}^{m \wedge \tau}\left\{e^{\lambda s}\left(\left|\Delta Y_{s}\right|^{2}+\left\|\Delta Z_{s}\right\|^{2}\right)\right.\right. \\
& \left.\left.+e^{\lambda s} \int_{\mathbb{R}_{0}^{n}}(\Delta K)^{2}(s, \zeta) \nu(d \zeta)\right\} d s\right] \\
& \leq 4 E\left[\int_{n \wedge \tau}^{\tau} e^{\lambda s}|g(s, \xi, \eta, \psi)|^{2} d s\right] .
\end{aligned}
$$


The last term in the above equation goes to zero as $n \rightarrow \infty$. Now, for $t \leq n$

$$
\begin{aligned}
\Delta Y_{t} & =\Delta Y_{n}+\int_{t \wedge \tau}^{n \wedge \tau}\left\{g\left(s, Y_{s}^{m}, Z_{s}^{m}, K_{s}^{m}\right)-g\left(s, Y_{s}^{n}, Z_{s}^{n}, K_{s}^{n}\right)\right\} d s \\
& -\int_{t \wedge \tau}^{n \wedge \tau} \Delta Z_{s} d B_{s}-\int_{t \wedge \tau}^{n \wedge \tau} \int_{\mathbb{R}_{0}^{n}} \Delta K(s, \zeta) \tilde{N}(d \zeta, d s) .
\end{aligned}
$$

Using the same argument as in the case of uniqueness, we have that

$$
\begin{aligned}
E\left[e^{\lambda t \wedge \tau}\left|\Delta Y_{t \wedge \tau}\right|^{2}\right] & \leq E\left[e^{\lambda t \wedge \tau}\left|\Delta Y_{n}\right|^{2}\right] \\
& \leq c E\left[\int_{n \wedge \tau}^{\tau} e^{\lambda s}\left|g\left(s, \xi_{s}, \eta_{s}, \psi_{s}\right)\right|^{2} d s\right] .
\end{aligned}
$$

It now follows that the sequence $\left(Y^{n}, Z^{n}, K^{n}\right)$ is Cauchy in the norm

$$
\begin{aligned}
\|(Y, Z, K)\|: & =E\left[\sup _{0 \leq t \leq \tau} e^{\lambda t}\left|Y_{t}\right|^{2}+\int_{0}^{\tau} e^{\lambda s}\left(\left|Y_{s}\right|^{2}+\left\|Z_{s}\right\|^{2}\right) d s\right. \\
& \left.+\int_{0}^{\tau} e^{\lambda s} \int_{\mathbb{R}_{0}^{n}} K^{2}(s, \zeta) \nu(d \zeta) d s\right] .
\end{aligned}
$$

So, we have that there is an unique solution to the BSDE (3.5)-(3.6), which satisfies for all $\lambda>2 \mu+K_{1}^{2}+K_{2}^{2}$, the condition

$$
\begin{aligned}
& E\left[\sup _{0 \leq t \leq \tau} e^{\lambda t}\left|Y_{t}\right|^{2}+\int_{0}^{\tau} e^{\lambda s}\left(\left|Y_{s}\right|^{2}+\left\|Z_{s}\right\|^{2}\right) d s+\int_{0}^{\tau} e^{\lambda s} \int_{\mathbb{R}_{0}^{n}} K^{2}(s, \zeta) \nu(d \zeta) d s\right] \\
& \leq c E\left[e^{\lambda \tau}|\xi|^{2}+\int_{0}^{\tau} e^{\lambda s}|g(s, 0,0,0)|^{2} d s\right] .
\end{aligned}
$$

\subsection{Optimal control with partial information and infinite horizon}

Now, let us get back to the problem of maximizing the performance functional

$$
J(u)=E\left[\int_{0}^{\infty} f(t, X(t), u(t)) d t\right]
$$

where $X(t)$ is of the form (3.1). Our aim is to find a $\hat{u} \in \mathcal{A}_{\varepsilon}$ such that

$$
J(\hat{u})=\sup _{u \in \mathcal{A}_{\varepsilon}} J(u)
$$


where $u(t)$ is our predictable control adapted to a subfiltration

$$
\mathcal{E}_{t} \subset \mathcal{F}_{t},
$$

with values in a set $U \subset \mathbb{R}^{n}$. Let $H$ be the Hamiltonian defined by (3.3) and $p$ the solution to the adjoint equation (3.4). Then we have the following maximum principle;

Theorem 3.3 (Sufficient Infinite Horizon Maximum Principle). Let $\hat{u} \in \mathcal{A}_{\varepsilon}$ and let $(\hat{p}(t), \hat{q}(t), \hat{r}(t, z))$ be an associated solution to the equation (3.4). Assume that for all $u \in \mathcal{A}_{\varepsilon}$ the following terminal condition holds:

$$
0 \leq E\left[\varlimsup_{t \rightarrow \infty}\left[\hat{p}(t)^{T}(X(t)-\hat{X}(t))\right]\right]<\infty
$$

Moreover, assume that $H(t, x, u, \hat{p}(t), \hat{q}(t), \hat{r}(t, \cdot))$ is concave in $x$ and $u$ and

$$
\begin{array}{r}
E\left[H(t, \hat{X}(t), \hat{u}(t), \hat{p}(t), \hat{q}(t), \hat{r}(t, \cdot)) \mid \mathcal{E}_{t}\right] \\
=\max _{u \in U} E\left[H(t, \hat{X}(t), u, \hat{p}(t), \hat{q}(t), \hat{r}(t, \cdot)) \mid \mathcal{E}_{t}\right] .
\end{array}
$$

In addition we assume that for all $T<\infty$,

$$
\begin{aligned}
& E\left[\int _ { 0 } ^ { T } ( \hat { X } ( t ) - X ^ { u } ( t ) ) ^ { T } \left[\hat{q} \hat{q}^{T}\right.\right. \\
& \left.\left.+\int_{\mathbb{R}_{0}^{n}} \hat{r} \hat{r}^{T}(t, z) \nu(d z)\right]\left(\hat{X}(t)-X^{u}(t)\right) d t\right]<\infty, \\
& E\left[\int _ { 0 } ^ { T } \hat { p } ( t ) ^ { T } \left[\sigma \sigma^{T}(t, X(t), u(t))\right.\right. \\
& \left.\left.+\int_{\mathbb{R}_{0}^{n}} \theta \theta^{T}(t, X(t), u(t), z) \nu(d z)\right] p(t) d t\right]<\infty, \\
& E\left[\left|\nabla_{u} H(t, \hat{X}(t), \hat{u}(t), \hat{p}(t), \hat{q}(t), \hat{r}(t, \cdot))\right|^{2}\right]<\infty,
\end{aligned}
$$

and that

$$
E\left[\int_{0}^{\infty}|H(s, X(s), u(s), \hat{p}(s), \hat{q}(s), \hat{r}(s, \cdot))|\right]<\infty
$$

for all $u$.

Then we have that $\hat{u}(t)$ is optimal.

Remark 3.4. Note that, since $p(t)$ has the economic interpretation as the marginal value of the resource (alternatively the shadow price if representing an outside resource), the requirement

$$
0 \leq E\left[\varlimsup_{t \rightarrow \infty}\left[\hat{p}(t)^{T}(X(t)-\hat{X}(t))\right]\right]<\infty
$$

has the economic interpretation that if the marginal value is positive at infinity we want to have 
as little resources left as possible.

Remark 3.5. The requirement in the finite horizon case that $p(T)=0$ does not translate into $\lim _{T \rightarrow \infty} p(T)=0$ as was shown in the deterministic case in Halkin [1974].

Proof. Let $I^{\infty}:=E\left[\int_{0}^{\infty}(f(t, X(t), u(t))-f(t, \hat{X}(t), \hat{u}(t))) d t\right]=J(u)-J(\hat{u})$. Then $I^{\infty}=$ $I_{1}^{\infty}-I_{2}^{\infty}-I_{3}^{\infty}-I_{4}^{\infty}$, where

$$
\begin{aligned}
& I_{1}^{\infty}:=E\left[\int_{0}^{\infty}(H(s, X(s), u(s), \hat{p}(s), \hat{q}(s), \hat{r}(s, \cdot))\right. \\
&-H(t, \hat{X}(s), \hat{u}(t), \hat{p}(s), \hat{q}(s), \hat{r}(s, \cdot))) d s], \\
& I_{2}^{\infty}:=E\left[\int_{0}^{\infty} \hat{p}(s)^{T}(b(s, X(s), u(s))-\hat{b}(s, \hat{X}(s), \hat{u}(s))) d s\right], \\
& I_{3}^{\infty}:=E\left[\int_{0}^{\infty} \operatorname{tr}\left[q(s)^{T}(\sigma(s, X(s), u(s))-\hat{\sigma}(s, \hat{X}(s), \hat{u}(s)))\right] d s\right],
\end{aligned}
$$

and

$$
\begin{aligned}
I_{4}^{\infty} & :=E\left[\int_{0}^{\infty} \sum_{i, j} \int_{\mathbb{R}_{0}^{n}}(\theta(s, X(s), u(s), z)\right. \\
& \left.-\hat{\theta}(s, \hat{X}(s), \hat{u}(s), z))^{T} \hat{r}_{i, j}(s, z) \nu_{j}(d z) d s\right] .
\end{aligned}
$$

Write $H_{t, X, u, \hat{p}, \hat{q}, \hat{r}}:=H(t, X(t), u(t), \hat{p}(t), \hat{q}(t), \hat{r}(t, \cdot))$ and similar for other combinations. We have from concavity that

$$
\begin{aligned}
& H_{t, X, u, \hat{p}, \hat{q}, \hat{r}}-H_{t, \hat{X}, \hat{u}, \hat{p}, \hat{q}, \hat{r}} \\
& \leq \nabla_{x} H(t, \hat{X}(t), \hat{u}(t), \hat{p}(t), \hat{q}(t), \hat{r}(t, \cdot))^{T}(X(t)-\hat{X}(t)) \\
& +\nabla_{u} H(t, \hat{X}(t), \hat{u}(t), \hat{p}(t), \hat{q}(t), \hat{r}(t, \cdot))^{T}(u(t)-\hat{u}(t)) .
\end{aligned}
$$

Then we have from $(3.16),(3.19)$ and that $u(t)$ is adapted to $\varepsilon_{t}$,

$$
\begin{aligned}
0 & \geq \nabla_{u} E\left[H_{t, \hat{X}, u, \hat{p}, \hat{q}, \hat{r}} \mid \mathcal{E}_{t}\right]_{u=\hat{u}(t)}^{T}(u(t)-\hat{u}(t)) \\
& =E\left[\nabla_{u} H_{t, \hat{X}, \hat{u}, \hat{p}, \hat{q}, \hat{r}}^{T}(u(t)-\hat{u}(t)) \mid \mathcal{E}_{t}\right] .
\end{aligned}
$$

Combining (3.4), (3.17), (3.21) and (3.22)

$$
\begin{aligned}
I_{1}^{\infty} & \leq E\left[\int_{0}^{\infty} \nabla_{x} H_{t, \hat{X}, \hat{u}, \hat{p}, \hat{q}, \hat{r}}^{T}(X(s)-\hat{X}(s)) d s\right] \\
& =E\left[\int_{0}^{\infty}(X(s)-\hat{X}(s))^{T} d \hat{p}(s)\right]=:-J_{1} .
\end{aligned}
$$


Now, using (3.15) and It $\bar{o}$ 's formula (for simplicity let $\left.\hat{\theta}_{s, X, u, z}:=\hat{\theta}(s, \hat{X}(s), \hat{u}(s), z)\right)$ and $\theta_{s, X, u, z}:=$ $\theta(s, \hat{X}(s), \hat{u}(s), z)))$

$$
\begin{aligned}
0 & \leq E\left[\overline{\lim _{t \rightarrow \infty}}\left[\hat{p}(t)^{T}(X(t)-\hat{X}(t))\right]=E\left[\overline { \operatorname { l i m } _ { t \rightarrow \infty } } \left[\int_{0}^{t} \hat{p}(s)^{T}\left(b_{s, X, u}-b_{s, \hat{X}, \hat{u}}\right) d s\right.\right.\right. \\
& +\int_{0}^{t} \hat{p}(s)^{T}(\sigma(s, X(s), u(s))-\hat{\sigma}(s, \hat{X}(s), \hat{u}(s))) d B(s) \\
& +\int_{0}^{t} \int_{\mathbb{R}_{0}^{n}} \hat{p}(s)^{T}(\theta(s, X(s), u(s), z)-\hat{\theta}(s, \hat{X}(s), \hat{u}(s), z)) \tilde{N}(d z, d s) \\
& +\int_{0}^{t}(X(s)-\hat{X}(s))^{T}\left(-\nabla_{x} \hat{H}(s, \hat{X}(s), \hat{u}(s), \hat{p}(s), \hat{q}(s), \hat{r}(s, \cdot))\right) d s \\
& +\int_{0}^{t} \hat{q}(s)^{T}(X(s)-\hat{X}(s)) d B(s)+\int_{0}^{t} \int_{\mathbb{R}_{0}^{n}} \hat{r}(s, z)(X(s)-\hat{X}(s)) \tilde{N}(d z, d s) \\
& +\int_{0}^{t} \operatorname{tr}\left[\hat{q}(s)^{T}(\sigma(s, X(s), u(s))-\hat{\sigma}(s, \hat{X}(s), \hat{u}(s)))\right] d s \\
& +\int_{0}^{t} \sum_{i, j} \int_{\mathbb{R}_{0}^{n}}\left(\theta_{s, X, u, z}-\hat{\theta}_{s, X, u, z}^{T} \hat{r}_{i, j}(s, z) \nu_{j}(d z) d s\right. \\
& +\int_{0}^{t} \int_{\mathbb{R}_{0}^{n}}\left(\theta_{s, X, u, z}-\hat{\theta}_{s, X, u, z}^{T} \hat{r}(s, z) \tilde{N}(d z, d s)\right]
\end{aligned}
$$


From (3.17), (3.18), we have that

$$
\begin{aligned}
0 & \leq E\left[\varlimsup _ { t \rightarrow \infty } \left[\int_{0}^{t} \hat{p}(s)^{T}\left(b_{s, X, u}-b_{s, \hat{X}, \hat{u}}\right) d s\right.\right. \\
& +\int_{0}^{t}(X(s)-\hat{X}(s))^{T}\left(-\nabla_{x} H_{t, \hat{X}, \hat{u}, \hat{p}, \hat{q}, \hat{r}}\right) d s \\
& +\int_{0}^{t} \operatorname{tr}\left[\hat{q}(s)^{T}(\sigma(s, X(s), u(s))-\hat{\sigma}(s, \hat{X}(s), \hat{u}(s)))\right] d s \\
& \left.\left.+\int_{0}^{t} \sum_{i, j} \int_{\mathbb{R}_{0}^{n}}\left(\theta_{s, X, u, z}-\theta_{s, \hat{X}, \hat{u}, z}\right)^{T} \hat{r}_{i, j}(s, z) \nu_{j}(d z) d s\right]\right] \\
& =E\left[\int_{0}^{\infty} \hat{p}(s)^{T}(b(s, X(s), u(s))-\hat{b}(s, X(s), u(s))) d s\right. \\
& +\int_{0}^{\infty}(X(s)-\hat{X}(s))^{T}\left(-\nabla_{x} H_{t, \hat{X}, \hat{u}, \hat{p}, \hat{q}, \hat{r}}\right) d s \\
& +\int_{0}^{\infty} \operatorname{tr}\left[\hat{q}(s)^{T}(\sigma(s, X(s), u(s))-\hat{\sigma}(s, \hat{X}(s), \hat{u}(s)))\right] d s \\
& \left.+\int_{0}^{\infty} \sum_{i, j} \int_{\mathbb{R}_{0}^{n}}\left(\theta_{s, X, u, z}-\theta_{s, \hat{X}, \hat{u}, z}\right)^{T} \hat{r}_{i, j}(s, z) \nu_{j}(d z) d s\right] \\
& =I_{1,2}^{\infty}+J_{1}^{\infty}+I_{1,3}^{\infty}+I_{1,4}^{\infty} .
\end{aligned}
$$

Finally, combining the above we get

$$
\begin{aligned}
J(u)-J(\hat{u}) & \leq I_{1}^{\infty}-I_{2}^{\infty}-I_{3}^{\infty}-I_{4}^{\infty} \\
& \leq-J_{1}^{\infty}-I_{2}^{\infty}-I_{3}^{\infty}-I_{4}^{\infty} \\
& \leq 0 .
\end{aligned}
$$

This holds for all $u \in \mathcal{A}_{\varepsilon}$, so the proof is complete.

\subsection{Examples}

Example 3.6 (Optimal Consumption Rate). Let

$$
J(u)=E\left[\int_{0}^{\infty} e^{-\rho t} \ln (u(t) X(t)) d t\right],
$$

where

$$
\begin{aligned}
d X(t) & =X(t)(\mu(t)-u(t)) d t+X(t) \sigma(t) d B(t) \\
X(0) & =x_{0}
\end{aligned}
$$


and $\rho \geq 0$. We have that

$$
X(t)=X_{0} \exp \left[\int_{0}^{t}\left[(\mu(s)-u(s))-\frac{1}{2} \sigma^{2}(s)\right] d s+\int_{0}^{t} \sigma(s) d B(s)\right] .
$$

Then we deal with the problem of maximizing $J(u)$ over all $u(t) \geq 0$. In this case the Hamiltonian takes the form

$$
H(t, x, u, p, q)=e^{-\rho t} \ln (u x)+x(\mu-u) p+x \sigma q,
$$

so that we get the partial derivatives

$$
\nabla_{x} H(t, x, u, p, q)=\frac{e^{-\rho t}}{x}+(\mu-u) p+\sigma q
$$

and

$$
\nabla_{u} H(t, x, u, p, q)=\frac{e^{-\rho t}}{u}-x p
$$

This gives us that

$$
-d p(t)=\left[\frac{e^{-\rho t}}{X(t)}+(\mu(t)-u(t)) p(t)+\sigma(t) q(t)\right] d t-q(t) d B(t) .
$$

so that

$$
\hat{u}(t)=\frac{e^{-\rho t}}{\hat{X}(t) \hat{p}(t)} .
$$

Let us try the infinite horizon BSDE with terminal condition $\lim _{t \rightarrow \infty} p(t)=0$,

$$
\begin{aligned}
-d p(t) & =\left[\frac{e^{-\rho t}}{X(t)}+(\mu(t)-u(t)) p(t)+\sigma(t) q(t)\right] d t-q(t) d B(t), \\
\lim _{t \rightarrow \infty} p(t) & =0 .
\end{aligned}
$$

Lemma 3.7 (Solution of infinite horizon linear BSDE with jumps). Let $A(t), \beta(t)$ and $\alpha(t, \zeta)$ be $\mathcal{F}_{t}$-predictable processes such that

$$
E\left[\int_{0}^{\infty}\left\{|A(t)|+\beta^{2}(t)+\int_{\mathbf{R}} \alpha^{2}(s, \zeta) \nu(d \zeta)\right\} d t\right]<\infty
$$

and define $\Gamma_{t, s}$ as the solution of the linear $S D E$

$$
\begin{aligned}
d \Gamma_{t, s} & =\Gamma_{t^{-}, s}\left(A(t) d t+\beta(t) d B(t)+\int_{\mathbb{R}_{0}^{n}} \alpha(t, \zeta) \bar{N}(d \zeta, d t)\right), s \geq t \geq 0, \\
\Gamma_{t, t} & =1 .
\end{aligned}
$$


Let $C(t)$ be a predictable process such that

$$
E\left[\int_{0}^{\infty} \Gamma_{0, s}|C(s)| d s\right]<\infty .
$$

Then a solution $(Y(t), Z(t), K(t, \zeta))$ of the linear BSDE

$$
\begin{aligned}
-d Y(t) & =\left[A(t) Y(t)+Z(t) \beta(t)+C(t)+\int_{\mathbb{R}_{0}^{n}} \alpha(t, \zeta) K(t, \zeta) d \nu(\zeta)\right] d t \\
& -Z(t) d B(t)-\int_{\mathbb{R}_{0}^{n}} K(t, \zeta) \bar{N}(d \zeta, d t), \\
\lim Y(t) & =0, t \rightarrow \infty,
\end{aligned}
$$

is given by

$$
Y(t)=E\left[\int_{t}^{\infty} \Gamma_{t, s} C(s) d s \mid \mathcal{F}_{t}\right], t \geq 0
$$

If in additon

$$
E\left[\int_{0}^{\infty} e^{\lambda t}|Y(t)|^{2} d t\right]<\infty
$$

where $\lambda$ as in (3.7), then $Y(t)$ is the unique solution.

Proof. By Itō's Lemma we have that

$$
\begin{aligned}
d\left(\Gamma_{0, t} Y_{t}\right) & =-\Gamma_{0, t} C_{t} d t+\Gamma_{0, t}\left(Z_{t}+Y_{t} \beta_{t}\right) d B_{t} \\
& +\int_{\mathbb{R}_{0}^{n}}\left[Y(t) \alpha(t, \zeta) \Gamma_{0, t}+K(t, \zeta) \Gamma_{0, t}+K(t, \zeta) \alpha(t, \zeta) \Gamma_{0, t}\right] \tilde{N}(d \zeta, d t) .
\end{aligned}
$$

So

$$
\begin{aligned}
\Gamma_{0, t} Y_{t} & +\int_{t}^{\infty} \Gamma_{0, s} C_{s} d s=\int_{t}^{\infty} \Gamma_{0, s}\left(Z_{s}+Y_{s} \beta_{s}\right) d B(s) \\
& +\int_{t}^{\infty} \int_{\mathbb{R}_{0}^{n}}\left[Y(s) \alpha(s, \zeta) \Gamma_{0, s}+K(s, \zeta) \Gamma_{0, s}+K(s, \zeta) \alpha(s, \zeta) \Gamma_{0, s}\right] \tilde{N}(d \zeta, d s) .
\end{aligned}
$$

By taking expectation we get the desired result. The uniqueness follows from Theorem 3.1.

From the above lemma we see that the solution of the linear, infinite horizon BSDE (3.23) (3.24) is

$$
\hat{p}(t)=E\left[\int_{t}^{\infty} \frac{\hat{\Gamma}_{s}}{\hat{\Gamma}_{t}} \frac{e^{-\rho s}}{\hat{X}_{s}} d s \mid \mathcal{F}_{t}\right],
$$

where

$$
\hat{\Gamma}_{t}=e^{\int_{0}^{t}\left[(\mu(s)-u(s))-\frac{1}{2} \sigma^{2}(s)\right] d s+\int_{0}^{t} \sigma(s) d B(s)}=\frac{\hat{X}(t)}{x_{0}} .
$$


Hence

$$
\hat{p}(t)=\frac{1}{\rho} e^{-\rho t} \frac{1}{\hat{X}(t)}
$$

and

$$
\varlimsup_{t \rightarrow \infty} \hat{p}(t)(X(t)-\hat{X}(t)) \geq \varlimsup_{t \rightarrow \infty} \hat{p}(t) X(t) \geq 0
$$

So

$$
\hat{u}(t)=\rho,
$$

is an optimal control.

Example 3.8 (Optimal portfolio). Let

$$
J(u)=E\left[\int_{0}^{\infty} e^{-\rho t} \ln (u(t) X(t)) d t\right]
$$

where

$$
\begin{aligned}
d X(t) & =X(t) \mu(t)(1-u(t)) d t+X(t) \sigma(t)(1-u(t)) d B(t) \\
X(0) & =x_{0}
\end{aligned}
$$

and $\rho \geq 0$. We have that

$$
\begin{aligned}
X(t) & =X_{0} \exp \left[\int_{0}^{t}\left[\mu(s)(1-u(s))-\frac{1}{2} \sigma^{2}(s)(1-u(t))^{2}\right] d s\right. \\
& \left.+\int_{0}^{t} \sigma(s)(1-u(s)) d B(s)\right] .
\end{aligned}
$$

Then we deal with the problem of maximizing $J(u)$ over all $u(t) \geq 0$. We have the Hamilton function takes the form

$$
H(t, x, u, p, q)=e^{-\rho t} \ln (u x)+x \mu(1-u) p+x \sigma(1-u) q,
$$

so that we get the partial derivatives

$$
\nabla_{x} H(t, x, u, p, q)=\frac{e^{-\rho t}}{x}+\mu(1-u) p+\sigma(1-u) q
$$

and

$$
\nabla_{u} H(t, x, u, p, q)=\frac{e^{-\rho t}}{u}-x \mu p-x \sigma q
$$

This gives us that

$$
-d p(t)=\left[\frac{e^{-\rho t}}{X(t)}+\mu(t)(1-u(t)) p(t)+\sigma(t)(1-u(s)) q(t)\right] d t-q(t) d B(t)
$$

So that

$$
\hat{u}(t)=\frac{e^{-\rho t}}{\hat{X}(t)(\mu \hat{p}(t)+\sigma \hat{q}(t))} .
$$


Let us try the infinite horizon BSDE with terminal condition $\lim _{t \rightarrow \infty} p(t)=0$, so that

$$
\begin{aligned}
-d p(t) & =\left[\frac{e^{-\rho t}}{X(t)}+\mu(t)(1-u(t)) p(t)+\sigma(t)(1-u(s)) q(t)\right] d t \\
& -q(t) d B(t) \\
\lim _{t \rightarrow \infty} p(t) & =0
\end{aligned}
$$

From the above lemma we see that the solution of the linear, infinite horizon BSDE (3.25) (3.26) is

$$
\hat{p}(t)=E\left[\int_{t}^{\infty} \frac{\hat{\Gamma}_{0, s}}{\hat{\Gamma}_{0, t}} \frac{e^{-\rho s}}{\hat{X}_{s}} d s \mid \mathcal{F}_{t}\right],
$$

where

$$
\begin{aligned}
\hat{\Gamma}_{t} & =\exp \left[\int_{0}^{t}\left[\mu(s)(1-u(s))-\frac{1}{2} \sigma^{2}(s)(1-u(s))^{2}\right] d s\right. \\
& \left.+\int_{0}^{t} \sigma(s)(1-u(s)) d B(s)\right] \\
& =\frac{\hat{X}(t)}{x_{0}} .
\end{aligned}
$$

Hence

$$
\hat{p}(t)=\frac{1}{\rho} e^{-\rho t} \frac{1}{\hat{X}(t)} .
$$

and

$$
\varlimsup_{t \rightarrow \infty} \hat{p}(t)(X(t)-\hat{X}(t)) \geq \varlimsup_{t \rightarrow \infty} \hat{p}(t) X(t) \geq 0 .
$$

Since

$$
\begin{aligned}
d\left(e^{-\rho t} \frac{1}{X(t)}\right) & =e^{-\rho t} \frac{1}{X(t)} d t-e^{-\rho t} \frac{1}{X(t)}(\mu(t)-u(t)) d t \\
& +e^{-\rho t} \frac{1}{X(t)} \sigma^{2}(t) d t+e^{-\rho t} \frac{1}{X(t)} \sigma(t) d B(t),
\end{aligned}
$$

we must have that

$$
\hat{q}(t)=\frac{1}{\rho} e^{-\rho t} \frac{1}{\hat{X}(t)} \sigma(t)
$$

So

$$
\hat{u}(t)=\frac{\rho}{\mu+\sigma},
$$

is an optimal control. 
Example 3.9 (Optimal consumption rate - part II). As above, let

$$
J(u)=E\left[\int_{0}^{\infty} e^{-\rho t} \ln (u(t) X(t)) d t\right] .
$$

But add a jump part

$$
\begin{aligned}
d X(t) & =X(t)(\mu(t)-u(t)) d t+X(t) \sigma(t) d B(t)+X(t) \int_{\mathbb{R}_{0}} \theta(t) z \tilde{N}(d z, d t) \\
X(0) & =x_{0}
\end{aligned}
$$

and we also add the assumption that we only know a subset of the information given by the market available at time $t$, represented by $\mathcal{E}_{t} \subset \mathcal{F}_{t}$. Let $\rho \geq 0$ be a constant. Then we deal with the problem of maximizing $J(u)$ over all $u(t) \geq 0$. We have

$$
\begin{gathered}
H(t, x, u, p, q, r)=e^{-\rho t} \ln (u x)+x(\mu-u) p+x \sigma q+x \int_{\mathbb{R}_{0}} \theta(t) z r(t, z) \nu(d z) \\
\nabla_{x} H(t, x, u, p, q, r)=\frac{e^{-\rho t}}{x}+(\mu-u) p+\sigma q+\int_{\mathbb{R}_{0}} \theta(t) z r(t, z) \nu(d z), \\
\nabla_{u} H(t, x, u, p, q, r)=\frac{e^{-\rho t}}{u}-x p
\end{gathered}
$$

and

$$
\begin{aligned}
-d p(t) & =\left[\frac{e^{-\rho t}}{X(t)}+(\mu(t)-u(t)) p(t)+\sigma(t) q(t)+\int_{\mathbb{R}_{0}} \theta(t) z r(t, z) \nu(d z)\right] d t \\
& -q(t) d B(t)-\int_{\mathbb{R}_{0}} \theta(t) z \tilde{N}(d z, d t) \\
\lim _{t \rightarrow \infty} p(t) & =0 .
\end{aligned}
$$

If we maximice

$$
E\left[H(t, \hat{X}(t), u, \hat{p}(t), \hat{q}(t), \hat{r}(t, \cdot)) \mid \mathcal{E}_{t}\right]
$$

we get that

$$
\begin{aligned}
\nabla_{u} E\left[H(t, \hat{X}(t), u, \hat{p}(t), \hat{q}(t)) \mid \mathcal{E}_{t}\right] & =E\left[\nabla_{u} H(t, \hat{X}(t), u, \hat{p}(t), \hat{q}(t)) \mid \mathcal{E}_{t}\right] \\
& =E\left[\frac{e^{-\rho t}}{u}-\hat{X}(t) \hat{p}(t) \mid \mathcal{E}_{t}\right] .
\end{aligned}
$$

Interchanging derivative and integration at $u$ is justified if in some neighbourhood of $u$ we have that

$$
\left|\nabla_{u} H(t, \hat{X}(t), u, \hat{p}(t), \hat{q}(t))(\omega)\right| \leq F(\omega, t)
$$

for some integrable function $F$. So following this we have that

$$
\hat{u}(t)=E\left[\frac{e^{-\rho t}}{\hat{X}(t) \hat{p}(t)} \mid \mathcal{E}_{t}\right] .
$$


The solution of the linear, infinite horizon BSDE (3.23) - (3.24) is (see Royer [2006])

$$
\hat{p}(t)=E\left[\int_{t}^{\infty} \frac{\hat{\Gamma}_{s}}{\hat{\Gamma}_{t}} \frac{e^{-\rho s}}{\hat{X}_{s}} d s \mid \mathcal{F}_{t}\right],
$$

where

$$
\begin{aligned}
& d \hat{\Gamma}_{t}=X(t)(\mu(t)-u(t)) d t+X(t) \sigma(t) d B(t)+X\left(t^{-}\right) \int_{\mathcal{R}_{0}} \theta(t) z \tilde{N}(d z, d t), \\
& X(0)=1
\end{aligned}
$$

So

$$
\hat{\Gamma}_{t}=\frac{\hat{X}(t)}{x_{0}} .
$$

Hence

$$
\hat{p}(t)=\frac{1}{x_{0} \hat{\Gamma}_{t}} \frac{1}{\rho} e^{-\rho t}=\frac{1}{\rho} e^{-\rho t} \frac{1}{\hat{X}(t)} .
$$

Therefore we have that

$$
\varlimsup_{t \rightarrow \infty} \hat{p}(t)(X(t)-\hat{X}(t))=\varlimsup_{t \rightarrow \infty} \hat{p}(t) X(t) \geq 0 .
$$

So

$$
\hat{u}(t)=E\left[\rho, \mid \mathcal{E}_{t}\right]
$$

is an optimal control.

Example 3.10 (Optimal Portfolio Selection With Consumption). For this example let us look at a market with two investment possibilities:

1. A bond or bank account with unit price

$$
d Z_{0}(t)=\rho Z_{0}(t) d t
$$

2. A stock with unit price

$$
d Z_{1}(t)=\mu Z_{1}(t) d t+\sigma Z_{1}(t) d B(t) .
$$

Here $\rho>0 \mu$ and $\sigma \neq 0$ are constants. Let $\left(Y_{0}, Y_{1}\right)$ denote the amount the agent has invested in the bonds and stocks respectively at time $t$. Consider then $\pi(t, \omega)=\pi(t)$, the fraction of the wealth invested in the stocks, e.g.

$$
\pi(t)=\frac{Z_{1}(t)}{Z_{0}(t)+Z_{1}(t)}
$$

Further let $\lambda(t, \omega)=\lambda(t)$ be the consumption rate relative to the wealth so that the investor controls

$$
u(t)=(\pi(t), \lambda(t)) .
$$

Then let

$$
J(u)=E\left[\int_{0}^{\infty} e^{-\delta(t)} \frac{(\lambda(t) X(t))^{\gamma}}{\gamma} d t\right]
$$


be a performance functional, where

$$
d X(t)=X(t)[(\rho+\pi(t)(\mu-\rho)-\lambda(t)) d t+\sigma \pi(t) d B(t)]
$$

and $\rho \geq 0$. We have that

$$
X(t)=x_{0} \exp \left[\int_{0}^{t}\left\{\rho+\pi(s)(\mu-\rho)-\lambda(s)-\frac{1}{2} \sigma^{2} \pi^{2}(s)\right\} d s+\int_{0}^{t} \sigma \pi(s) d B(s)\right] .
$$

Then we want to maximize $J(u)$ over all $u=(\pi(t), \lambda(t)), \lambda \geq 0$. The Hamiltonian is

$$
H(t, x, u, p, q)=e^{-\delta t} \frac{(\lambda(t) X(t))^{\gamma}}{\gamma}+x(\rho+\pi(\mu-\rho)-\lambda) p+x \sigma \pi q,
$$

so that

$$
\nabla_{x} H(t, x, u, p, q)=e^{-\delta t} \lambda^{\gamma} x^{\gamma-1}+(\rho+\pi(\mu-\rho)-\lambda) p+\sigma \pi q .
$$

Further, we also have

$$
\begin{aligned}
-d p(t) & =\left[e^{-\delta t} \lambda^{\gamma}(t) X^{\gamma-1}(t)+(\rho+\pi(t)(\mu-\rho)-\lambda(t)) p(t)+\sigma \pi(t) q(t)\right] d t \\
& -q(t) d B(t) .
\end{aligned}
$$

and

$$
\nabla_{\lambda} H(t, x, u, p, q)=e^{-\delta t}(\lambda(t))^{\gamma-1} X^{\gamma}-x p .
$$

So that

$$
q(t)=-\frac{(\mu-\rho)}{\sigma} p(t)
$$

and

$$
\hat{\lambda}=\frac{1}{x} p^{\frac{1}{\gamma-1}} e^{\frac{\delta t}{\gamma-1}}
$$

Then

$$
\begin{aligned}
d p(t) & =-e^{\frac{\delta t}{\gamma-1}} \frac{1}{X(t)} p^{\frac{\gamma}{\gamma-1}}(t) d t-\left[\rho+\pi(t)(\mu-\rho)-\frac{1}{X(t)} p^{\frac{1}{\gamma-1}} e^{\frac{\delta t}{\gamma-1}}\right] p(t) d t \\
& +\sigma \pi(t) \frac{(\mu-\rho)}{\sigma} p(t) d t-\frac{(\mu-\rho)}{\sigma} p(t) d B(t) \\
& =-\rho p(t) d t-\frac{(\mu-\rho)}{\sigma} p(t) d B(t) .
\end{aligned}
$$


So to ensure that the requirement

$$
E\left[\varlimsup_{t \rightarrow \infty} \hat{p}(t)(X(t)-\hat{X}(t))\right] \geq 0,
$$

is satisfied it suffices that

$$
E\left[\varlimsup_{t \rightarrow \infty} \hat{p}(t) \hat{X}(t)\right] \leq 0
$$

Let us try to choose

$$
\hat{\lambda}(s)=\hat{\lambda}>0 \text { (constant) }
$$

and

$$
\hat{\pi}(s)=\hat{\pi}>0 \text { (constant) }
$$

Then by (3.33) we get

$$
\begin{aligned}
& p(t)=\hat{\lambda}^{(\gamma-1)} e^{-\delta t} \hat{X}^{(\gamma-1)}(t) \\
& =x_{0} \hat{\lambda}^{(\gamma-1)} \exp \left[\int_{0}^{t}\left\{-\delta+\hat{\pi}(\mu-\rho)-\hat{\lambda}-\frac{1}{2} \sigma^{2} \hat{\pi}^{2}\right\}(\gamma-1) d s\right] \\
& \exp \left[\int_{0}^{t} \sigma \hat{\pi}(\gamma-1) d B(s)\right]
\end{aligned}
$$

Comparing (3.36) with (3.34) we get

$$
-\rho-\frac{1}{2}\left(\frac{\mu-\rho}{\sigma}\right)^{2}=\left\{-\delta+\hat{\pi}(\mu-\rho)-\hat{\lambda}-\frac{1}{2} \sigma^{2} \hat{\pi}^{2}\right\}(\gamma-1)
$$

and

$$
-\frac{\mu-\rho}{\sigma}=\sigma \hat{\pi}(\delta-1) .
$$

By (3.38) we get

$$
\hat{\pi}=\frac{\mu-\rho}{\sigma^{2}(1-\gamma)}
$$

Substituted into (3.37) this gives

$$
\hat{\lambda}=\frac{1}{2} \frac{(\mu-\rho)^{2} \gamma}{\sigma^{2}(1-\gamma)^{2}}-\delta-\frac{\rho}{1-\gamma}
$$


provided that

$$
\frac{1}{2} \frac{(\mu-\rho)^{2} \gamma}{\sigma^{2}(1-\gamma)^{2}}-\delta-\frac{\rho}{1-\gamma}>0
$$

It remains to verify that with $\pi=\hat{\pi}, \lambda=\hat{\lambda}$ we have

$$
E\left[\varlimsup_{t \rightarrow \infty} \hat{p}(t) \hat{X}(t)\right] \leq 0
$$

By (3.27) and (3.34) we have

$$
\begin{aligned}
& p(t) \hat{X}(t)=p(0) x_{0} \exp \left[\int _ { 0 } ^ { t } \left\{-\rho-\frac{1}{2}\left(\frac{\mu-\rho}{\sigma}\right)^{2}\right.\right. \\
& \left.+\rho+\frac{(\mu-\rho)^{2}}{\sigma^{2}(1-\gamma)}-\hat{\lambda}-\frac{1}{2} \frac{(\mu-\rho)^{2}}{\sigma^{2}(1-\gamma)^{2}}\right\} d s \\
& \left.+\int_{0}^{t}\left\{-\frac{\mu-\rho}{\sigma}+\frac{\mu-\rho}{\sigma(1-\gamma)}\right\} d B(s)\right] \\
& =p(0) x_{0} \exp \left[\int _ { 0 } ^ { t } \left\{-\frac{1}{2}\left(\frac{\mu-\rho}{\sigma}\right)^{2}+\frac{(\mu-\rho)^{2}}{\sigma^{2}(1-\gamma)}\right.\right. \\
& \left.\left.-\frac{1}{2} \frac{(\mu-\rho)^{2} \gamma}{\sigma^{2}(1-\gamma)^{2}}+\delta+\frac{\rho}{1-\gamma}-\frac{1}{2} \frac{(\mu-\rho)^{2}}{\sigma^{2}(1-\gamma)^{2}}\right\} d s+\int_{0}^{t} \frac{(\mu-\rho) \gamma}{\sigma(1-\gamma)} d B(s)\right] \\
& =p(0) x_{0} \exp \left[\left(\frac{-\gamma(\gamma+1)}{2(1-\gamma)^{2}}+\delta+\frac{\rho}{1-\gamma}\right) t+\frac{(\mu-\rho) \gamma}{\sigma(1-\gamma)} B(t)\right]
\end{aligned}
$$

Therfore (3.42) holds iff

$$
-\frac{\gamma(\gamma+1)}{2(1-\gamma)^{2}}+\delta+\frac{\rho}{1-\gamma}<0
$$

We have proved:

Theorem 3.11. Suppose (3.41) and (3.43) holds. Then the optimal porfolio $\hat{\pi}$ and the optimal relative consumption rate $\hat{\lambda}$ are given by (3.39) and (3.40), respectively.

\subsection{Necessary Maximum Principle}

To answer the question: if $\hat{u}$ is optimal does it satisfy

$$
\begin{array}{r}
E\left[H(t, \hat{X}(t), \hat{u}(t), \hat{p}(t), \hat{q}(t), \hat{r}(t, \cdot)) \mid \mathcal{E}_{t}\right] \\
=\max _{u \in U} E\left[H(t, \hat{X}(t), u, \hat{p}(t), \hat{q}(t), \hat{r}(t, \cdot)) \mid \mathcal{E}_{t}\right],
\end{array}
$$

we assume the following: 
A1 For all $t, h$ such that $0 \leq t<t+h \leq T$, all $i=1, \ldots, k$ and for all bounded $\varepsilon_{t}$-measurable $\alpha=\alpha(\omega)$, the control $\bar{\beta}(s):=\left(0, \ldots, \beta_{i}(s), 0, \ldots, 0\right) \in U \subset \mathbb{R}^{k}$ with

$$
\beta(s):=\alpha_{i} \mathbf{1}_{[t, t+h]}(s),
$$

belongs to $\mathcal{A}_{\varepsilon}$. Here

$$
\mathbf{1}_{[t, t+h]}(s)= \begin{cases}1 & \text { if } s \in[t, t+h] \\ 0 & \text { otherwise }\end{cases}
$$

A2 For all $u, \beta \in \mathcal{A}_{\varepsilon}$ with $\beta$ bounded, there exists $\delta>0$ such that $\hat{u}+\epsilon \beta \in \mathcal{A}_{\varepsilon}$ for all $\epsilon \in(-\delta, \delta)$.

A3 Given $u, \beta \in \mathcal{A}_{\varepsilon}$ with $\beta$ bounded, the process $Y(t)=Y^{(u, \beta)}(t)$ defined by

$$
Y(t)=\left.\frac{d}{d \epsilon} X^{\hat{u}+\epsilon \beta}(t)\right|_{\epsilon=0}=\left(Y_{1}(t), \ldots, Y_{n}(t)\right)^{T},
$$

exists and we have $Y(0)=0$ and

$$
\begin{aligned}
d Y_{i}(t) & =\lambda_{i}(t) d t+\sum_{j=1}^{n} \xi_{i j}(t) d B_{j}(t) \\
& +\sum_{j=1}^{n} \int_{\mathbb{R}_{0}^{n}} \zeta_{i j}(t, z) \tilde{N}_{j}(d z, d t),
\end{aligned}
$$

where

$$
\begin{aligned}
\lambda_{i}(t) & =\left(\nabla_{x} b_{t, X, u}^{i}\right)^{T} Y(t)+\left(\nabla_{u} b_{t, X, u}^{i}\right)^{T} \beta(t), \\
\xi^{i j}(t) & =\left(\nabla_{x} \sigma_{t, X, u}^{i j}\right)^{T} Y(t)+\left(\nabla_{u} \sigma_{t, X, u}^{i j}\right)^{T} \beta(t), \\
\zeta^{i j}(t, z) & =\left(\nabla_{x} \theta_{t, X, u, z}^{i j}\right)^{T} Y(t)+\left(\nabla_{u} \theta_{t, X, u, z}^{i j}\right)^{T} \beta(t) .
\end{aligned}
$$

A4 Assume that $\mathrm{f}$ satisfies a Lipschitz condition of the form

$$
\left|f\left(t, x_{1}, u_{1}\right)-f\left(t, x_{2}, u_{2}\right)\right| \leq C(t)\left(\left|x_{1}-x_{2}\right|+\left|u_{1}-u_{2}\right|\right),
$$

for any $t, x_{i}, u_{i}, i=1,2$.

We can then give an answer to the question.

Theorem 3.12 (Partial Information Necessary Maximum Principle). Suppose $\hat{u} \in \mathcal{A}_{\mathcal{E}}$ is a local maximum for $J(u)$, meaning that for all bounded $\beta \in \mathcal{A}_{\mathcal{E}}$ there exists a $\delta>0$ such that $\hat{u}+\epsilon \beta \in \mathcal{A}_{\varepsilon}$ for all $\epsilon \in(-\delta, \delta)$ and

$$
h(\epsilon):=J(\hat{u}+\epsilon \beta), \epsilon \in(-\delta, \delta)
$$


is maximal at $\epsilon=0$. Let $(\hat{p}(t), \hat{q}(t), \hat{r}(t, z))$ be the solution to the (linear) adjoint equation

$$
\begin{aligned}
d \hat{p}(t) & =-\nabla_{x} H(t, \hat{X}(t), \hat{u}(t), \hat{p}(t), \hat{q}(t), \hat{r}(t, \cdot)) d t \\
& +\hat{q}(t) d B(t)+\int_{\mathbb{R}_{0}^{n}} \hat{r}(z, t) \tilde{N}(d z, d t) .
\end{aligned}
$$

Moreover assume that if $\hat{Y}(t)=Y^{(\hat{u}, \beta)}(t)$, with corresponding coefficients $\hat{\lambda}_{i}, \hat{\xi_{i j}}, \hat{\zeta_{i j}}$, we have

$$
\begin{gathered}
\lim _{T \rightarrow \infty} E\left[\hat{p}(T)^{T} \hat{Y}(T)\right]=0, \\
E\left[\int_{0}^{\infty} C(t)(1+|\hat{Y}(t)|) d t\right]<\infty \\
E\left[\int_{0}^{T}\left\{\hat{Y}(t)^{T}\left[\hat{q} \hat{q}^{T}(t)+\int_{\mathbb{R}_{0}^{n}} \hat{r} \hat{r}^{T}(t, z) \nu(d z)\right] \hat{Y}(t)\right\} d t\right]<\infty,
\end{gathered}
$$

and

$$
\begin{aligned}
& E\left[\int _ { 0 } ^ { T } \hat { p } ^ { T } ( t ) \left[\hat{\xi} \hat{\xi}^{T}(t, \hat{X}(t), \hat{u}(t))\right.\right. \\
& \left.\left.+\int_{\mathbb{R}_{0}^{n}} \hat{\zeta} \hat{\zeta}^{T}(t, \hat{X}(t), \hat{u}(t), z) \nu(d z)\right] \hat{p}(t) d t\right]<\infty .
\end{aligned}
$$

for all $T<\infty$. Then $\hat{u}$ is a stationary point for $E[H \mid \mathcal{E}]$ in the sense that for all $t \geq 0$,

$$
E\left[\frac{\partial}{\partial u} H(t, \hat{X}(t), \hat{u}(t), \hat{p}(t), \hat{q}(t), \hat{r}(t, \cdot)) \mid \varepsilon_{t}\right]=0 .
$$

Proof. For simplicity we consider only the 1-dimensional case. First note that by A3,A4 and (3.46) we have that

$$
\begin{aligned}
& \left.\frac{\partial}{\partial s} J(\hat{u}+s \beta)\right|_{s=0}=\left.\frac{\partial}{\partial s} E\left[\int_{0}^{\infty} f\left(t, X^{\hat{u}+s \beta}(t), \hat{u}(t)+s \beta(t)\right) d t\right]\right|_{s=0} \\
& =\lim _{s \rightarrow 0} \frac{1}{s} E\left[\int_{0}^{\infty}\left\{f\left(t, X^{\hat{u}+s \beta}(t), \hat{u}(t)+s \beta(t)\right)-f\left(t, X^{\hat{u}}(t), \hat{u}(t)\right)\right\} d t\right] \\
& =E\left[\int_{0}^{\infty}\left\{\frac{\partial f}{\partial x}\left(t, X^{\hat{u}}(t), \hat{u}(t)\right) \hat{Y}(t)+\frac{\partial f}{\partial u}\left(t, X^{\hat{u}}(t), \hat{u}(t)\right) \beta(t)\right\} d t\right] .
\end{aligned}
$$


By (3.45), the Itō formula, A2, and (3.47) - (3.48), we have

$$
\begin{aligned}
0 & =\lim _{T \rightarrow \infty} E[\hat{p}(T) \hat{Y}(T)] \\
& =\lim _{T \rightarrow \infty} E\left[\int_{0}^{T} \hat{p}(t)\left\{\frac{\partial b}{\partial x}(t) \hat{Y}(t)+\frac{\partial b}{\partial u}(t) \beta(t)\right\} d t\right. \\
& +\int_{0}^{T} \hat{Y}(t)\left(-\frac{\partial \hat{H}}{\partial x}(t)\right) d t+\int_{0}^{T} \hat{q}(t)\left\{\frac{\partial \sigma}{\partial x}(t) \hat{Y}(t)+\frac{\partial \sigma}{\partial u}(t) \beta(t)\right\} d t \\
& \left.+\int_{0}^{T} \int_{\mathbb{R}} \hat{r}(t, \zeta)\left\{\frac{\partial \gamma}{\partial x}(t, \zeta) \hat{Y}(t)+\frac{\partial \gamma}{\partial u}(t, \zeta) \beta(t)\right\} \nu(d \zeta) d t\right] \\
& =\lim _{T \rightarrow \infty} E\left[\int _ { 0 } ^ { T } \hat { Y } ( t ) \left\{\frac{\partial b}{\partial x}(t) \hat{p}(t)\right.\right. \\
& \left.+\frac{\partial \sigma}{\partial x}(t) \hat{q}(t)+\int_{\mathbb{R}} \frac{\partial \gamma}{\partial x}(t, \zeta) \hat{r}(t, \zeta) \nu(d \zeta)-\frac{\partial \hat{H}}{\partial x}(t)\right\} d t \\
+ & \left.\int_{0}^{T} \beta(t)\left\{\frac{\partial b}{\partial u}(t) \hat{p}(t)+\frac{\partial \sigma}{\partial u}(t) \hat{q}(t)+\int_{\mathbb{R}} \frac{\partial \gamma}{\partial u}(t, \zeta) \hat{r}(t, \zeta) \nu(d \zeta)\right\} d t\right] \\
& =\lim _{T \rightarrow \infty} E\left[\int_{0}^{T} \hat{Y}(t)\left(-\frac{\partial f}{\partial x}(t)\right) d t+\int_{0}^{T} \beta(t)\left\{\frac{\partial \hat{H}}{\partial u}(t)-\frac{\partial f}{\partial u}(t)\right\} d t\right] \\
& =-\lim _{T \rightarrow \infty} E\left[\int_{0}^{T}\left\{\frac{\partial f}{\partial x}(t) \hat{Y}(t)+\frac{\partial f}{\partial u}(t) \beta(t)\right\} d t+\lim _{T \rightarrow \infty} E\left[\int_{0}^{T} \frac{\partial H}{\partial u}(t) \beta(t) d t\right] .\right.
\end{aligned}
$$

Hence

$$
\left.\frac{\partial}{\partial s} J(\hat{u}+s \beta)\right|_{s=0}=\lim _{T \rightarrow \infty} E\left[\int_{0}^{T} \frac{\partial H}{\partial u}(t) \beta(t) d t\right] .
$$

Define $\beta(s):=\alpha \mathbf{1}_{[t, t+h]}(s)$. Then

$$
E\left[\int_{t}^{t+h} \frac{\partial}{\partial u} H(t, \hat{X}(t), \hat{u}(t), \hat{p}(t), \hat{q}(t), \hat{r}(t, \cdot))^{T} \alpha d t\right]=0 .
$$

Differentiating with respect to $h$ at $h=0$ gives

$$
E\left[\frac{\partial}{\partial u} H(t, \hat{X}(t), \hat{u}(t), \hat{p}(t), \hat{q}(t), \hat{r}(t, \cdot)) \alpha\right]=0 .
$$

Since this holds for all $\mathcal{E}_{t}$ measurable $\alpha$, we have that

$$
E\left[\frac{\partial}{\partial u} H(t, \hat{X}(t), \hat{u}(t), \hat{p}(t), \hat{q}(t), \hat{r}(t, \cdot)) \mid \mathcal{E}_{t}\right]=0,
$$

which proves the theorem. 


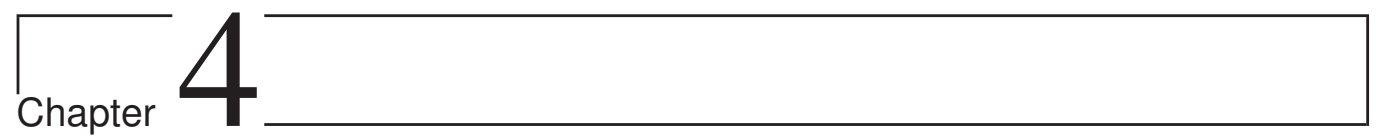

A Maximum Principle for Infinite Horizon Delay Equations

With Nacira Agram, Bernt Øksendal and Frank Proske. Published in SIAM Journal on Mathematical Analysis (Vol. 45, Issue 4). 


\subsection{Introduction}

To solve the stochastic control problems, there are two approaches: The dynamic programming method (HJB equation) and the maximum principle.

In this paper, our system is governed by the stochastic differential delay equation (SDDE in short):

$$
\begin{cases}d X(t)=b(t, X(t), Y(t), A(t), u(t)) d t & \\ +\sigma(t, X(t), Y(t), A(t), u(t)) d B(t) & \\ +\int_{\mathbb{R}_{0}} \theta(t, X(t), Y(t), A(t), u(t), z) \tilde{N}(d t, d z) ; & t \in[0, \infty), \\ X(t)=X_{0}(t) ; & t \in[-\delta, 0], \\ Y(t)=X(t-\delta) ; & t \in[0, \infty), \\ A(t)=\int_{t-\delta}^{t} e^{-\lambda(t-r)} X(r) d r ; & t \in[0, \infty),\end{cases}
$$

with a corresponding performance functional;

$$
J(u)=E\left[\int_{0}^{\infty} f(t, X(t), Y(t), A(t), u(t)) d t\right],
$$

where $u(t)$ is the control process.

The SDDE is not Markovian so we cannot use the dynamic programming method. However, we will prove stochastic maximum principles for this problem. A sufficient maximum principle in infinite horizon with the trivial transversality conditions where treated by Haadem et al. [2013]. The 'natural' transversality condition in the infinite case would be a zero limit condition, meaning in the economic sense that one more unit of good at the limit gives no additional value. But this property is not necessarily verified. In fact Halkin [1974] provides a counterexample for a 'natural' extension of the finite-horizon transversality conditions. Thus some care is needed in the infinite horizon case. For the case of the 'natural' transversality condition the discounted control problem was studied by Maslowski and Veverka [2011].

In real life, delay occurs everywhere in our society. For example this is the case in biology where the population growth depends not only on the current population size but also on the size some time ago. The same situation may occur in many economic growth models.

The stochastic maximum principle with delay has been studied by many authors. For example, Elsanosi et al. [2000] proved a verification theorem of variational inequality. Øksendal and Sulem [2007] established the sufficient maximum principle for certain class of stochastic control systems with delay in the state variable. In Haadem et al. [2013] an inifinite horizon system is studied, but without delay. In Chen and Wu [2010], a finite horizon version of a stochastic maximum principle for a system with delay both in the state variable and the control variable is derived. In Øksendal et al. [2011] a maximum principle for systems with delay is studied in the finite horizon case. However, to our knowledge, no one has studied the infinite horizon case for delay equations.

For backward differential equations see Situ [2002] and Li and Peng [2009]. For the infinite horizon BSDE see Peng and Shi [2000], Pardoux [1999], Yin [2008], Barles et al. [2009] and Royer [2006]. For more details about jump diffusion markets see Øksendal and Sulem [2008] and for background and details about stochastic fractional delay equations see Mohammed and Scheutzow [1997].

In this work, we establish two sufficient maximum principles and one necessary for the stochastic delay systems on infinite horizon with jumps.

Our paper is organized as follows: In the second section, we formulate the problem. The third section is devoted to the first and second sufficient maximum principles with an application 
to the optimal consumption rate from an economic quantity described by a stochastic delay equation. In the fourth section, we formulate a necessary maximum principle and we prove an existence and uniqueness of the advanced backward stochastic differential equations on infinite horizon with jumps in the last section.

\subsection{Formulation of the problem}

Let $(\Omega, \mathcal{F}, P)$ be a probability space with filtration $\left(\mathcal{F}_{t}\right)_{t>0}$ satisfying the usual conditions, on which an $\mathbb{R}$-valued standard Brownian motion $B($.$) and an independent compensated Poisson$ random measure $\tilde{N}(d t, d z)=N(d t, d z)-\nu(d z) d t$ are defined.

We consider the following stochastic control system with delay :

$$
\left\{\begin{aligned}
d X(t) & =b(t, X(t), Y(t), A(t), u(t)) d t \\
& +\sigma(t, X(t), Y(t), A(t), u(t)) d B(t) \\
& +\int_{\mathbb{R}_{0}} \theta(t, X(t), Y(t), A(t), u(t), z) \tilde{N}(d t, d z) ; t \in[0, \infty), \\
X(t) & =X_{0}(t) ; t \in[-\delta, 0], \\
Y(t) & =X(t-\delta) ; t \in[0, \infty), \\
A(t) & =\int_{t-\delta}^{t} e^{-\lambda(t-r)} X(r) d r ; t \in[0, \infty),
\end{aligned}\right.
$$

where $X_{0}(t)$ is a given continuous (deterministic) function, and

$$
\begin{aligned}
& \delta>0, \lambda>0 \text { are given constants. } \\
& b:[0, \infty) \times \mathbb{R} \times \mathbb{R} \times \mathbb{R} \times \mathcal{U} \times \Omega \rightarrow \mathbb{R} \\
& \sigma:[0, \infty) \times \mathbb{R} \times \mathbb{R} \times \mathbb{R} \times \mathcal{U} \times \Omega \rightarrow \mathbb{R} \\
& \theta:[0, \infty) \times \mathbb{R} \times \mathbb{R} \times \mathbb{R} \times \mathcal{U} \times \mathbb{R}_{0} \times \Omega \rightarrow \mathbb{R}
\end{aligned}
$$

are given continuous functions such that for all $t, b(t, x, y, a, u), \sigma(t, x, y, a, u)$ and $\theta(t, x, y, a, u, z)$ are $\mathcal{F}_{t}$-measurable for all $x \in \mathbb{R}, y \in \mathbb{R}, a \in \mathbb{R}, u \in \mathcal{U}$ and $z \in \mathbb{R}_{0}:=\mathbb{R} \backslash\{0\}$. We assume that $b, \sigma, \theta$ are $C^{1}$ (i.e. continuously differentiable/ Frèchet differentiable) with respect to $x, y, a, u$ and $z$, for all $t$ and a.a $\omega$. Let $\mathcal{E}_{t} \subset \mathcal{F}_{t}$ be a given subfiltration, representing the information available to the controller at time $t$. Let $\mathcal{U}$ be a non-empty subset of $\mathbb{R}$. We let $\mathcal{A}_{\varepsilon}$ denote a given family of admissible $\mathcal{E}_{t}$-adapted control processes. An element of $\mathcal{A}_{\mathcal{\varepsilon}}$ is called an admissible control. The corresponding performance functional is

$$
J(u)=E\left[\int_{0}^{\infty} f(t, X(t), Y(t), A(t), u(t)) d t\right] ; u \in \mathcal{A}_{\mathcal{E}}
$$

where we assume that

$$
E \int_{0}^{\infty}\left\{|f(t, X(t), Y(t), A(t), u(t))|+\left|\frac{\partial f}{\partial x_{i}}(t, X(t), Y(t), A(t), u(t))\right|^{2}\right\} d t<\infty .
$$


We also assume that $f$ is $C^{1}$ with respect to $x, y, a, u$ for all $t$ and a.a. $\omega$. The value function $\Phi$ is defined as

$$
\Phi\left(X_{0}\right)=\sup _{u \in \mathcal{A}_{\varepsilon}} J(u) .
$$

An admissible control $u^{*}$ is called an optimal control for (4.3) if it attains the maximum of $J(u)$ over $\mathcal{A}_{\mathcal{E}} \cdot(4.3)$ is called the state equation, the solution $X^{*}(t)$ corresponding to $u^{*}$ is called an optimal trajectory.

\subsection{A sufficient maximum principle}

In this section our objective is to establish a sufficient maximum principle.

\subsubsection{Hamiltonian and time-advanced BSDEs for adjoint equations}

We now introduce the adjoint equations and the Hamiltonian function for our problem. The Hamiltonian is defined by

$$
\begin{aligned}
& H(t, x, y, a, u, p, q, r(\cdot))=f(t, x, y, a, u)+b(t, x, y, a, u) p+\sigma(t, x, y, a, u) q \\
&+\int_{\mathbb{R}_{0}} \theta(t, x, y, a, u, z) r(z) \nu(d z),
\end{aligned}
$$

where

$$
H:[0, \infty) \times \mathbb{R} \times \mathbb{R} \times \mathbb{R} \times \mathcal{U} \times \mathbb{R} \times \mathbb{R} \times \Re \times \Omega \rightarrow \mathbb{R}
$$

and $\Re$ is the set of functions $r: \mathbb{R}_{0} \rightarrow \mathbb{R}$ such that the integral term in (4.7) converges and $\mathcal{U}$ is the set of possible control values.

We suppose that $b, \sigma$ and $\theta$ are $C^{1}$ functions with respect to $(x, y, a, u)$ and that

$$
\begin{gathered}
E\left[\int _ { 0 } ^ { \infty } \left\{\left|\frac{\partial b}{\partial x_{i}}(t, X(t), Y(t), A(t), u(t))\right|^{2}+\left|\frac{\partial \sigma}{\partial x_{i}}(t, X(t), Y(t), A(t), u(t))\right|^{2}\right.\right. \\
\left.\left.+\int_{\mathbb{R}_{0}}\left|\frac{\partial \theta}{\partial x_{i}}(t, X(t), Y(t), A(t), u(t))\right|^{2} \nu(d z)\right\} d t\right]<\infty
\end{gathered}
$$

for $x_{i}=x, y, a$ and $u$. tion

The adjoint processes $(p(t), q(t), r(t, \cdot)), t \in[0, \infty), z \in \mathbb{R}_{0}$ are assumed to satisfy the equa-

$$
d p(t)=E\left[\mu(t) \mid \mathcal{F}_{t}\right] d t+q(t) d B(t)+\int_{\mathbb{R}_{0}} r(t, z) \tilde{N}(d t, d z) ; t \in[0, \infty)
$$

where 


$$
\begin{aligned}
& \mu(t)=-\frac{\partial H}{\partial x}(t, X(t), Y(t), A(t), u(t), p(t), q(t), r(t, \cdot)) \\
& -\frac{\partial H}{\partial y}(t+\delta, X(t+\delta), Y(t+\delta), A(t+\delta), u(t+\delta), p(t+\delta), q(t+\delta), r(t+\delta, \cdot)) \\
& -e^{\lambda t}\left(\int_{t}^{t+\delta} \frac{\partial H}{\partial a}(s, X(s), Y(s), A(s), u(s), p(s), q(s), r(s, \cdot)) e^{-\lambda s} d s\right) .
\end{aligned}
$$

Remark 4.1. Note that we do not require a priori that the solution of (4.9)-(4.10) is unique.

The following result is an infinite horizon version of Theorem 3.1 in Øksendal et al. [2011].

\subsubsection{A first sufficient maximum principle}

Theorem 4.2. Let $\hat{u} \in \mathcal{A}_{\mathcal{E}}$ with corresponding state processes $\hat{X}(t), \hat{Y}(t)$ and $\hat{A}(t)$ and adjoint processes $\hat{p}(t), \hat{q}(t)$ and $\hat{r}(t, \cdot)$ assumed to satisfy the advanced backward stochastic differential equation (ABSDE in short) (4.9) - (4.10). Suppose that the following assertions hold:

(i)

$$
\varlimsup_{T \rightarrow \infty} E[\hat{p}(T)(X(T)-\hat{X}(T))] \geq 0,
$$

for all $u \in \mathcal{A}_{\varepsilon}$ with corresponding solution $X(t)$.

(ii) The function

$$
(x, y, a, u) \rightarrow H(t, x, y, a, u, \hat{p}(t), \hat{q}(t), \hat{r}(t, \cdot)),
$$

is concave for each $t \in[0, \infty)$ a.s.

(iii)

$$
E\left[\int_{0}^{T}\left\{\hat{q}^{2}(t)(\sigma(t)-\hat{\sigma}(t))^{2}+\int_{\mathbb{R}} \hat{r}^{2}(t, z)(\theta(t, z)-\hat{\theta}(t, z))^{2} \nu(d z)\right\} d t\right]<\infty
$$

for all $T<\infty$.

(iiii)

$$
\begin{aligned}
& \max _{v \in \mathcal{U}} E\left[H(t, \hat{X}(t), \hat{X}(t-\delta), \hat{A}(t), v, \hat{p}(t), \hat{q}(t), \hat{r}(t, \cdot)) \mid \mathcal{E}_{t}\right] \\
& =E\left[H(t, \hat{X}(t), \hat{X}(t-\delta), \hat{A}(t), \hat{u}(t), \hat{p}(t), \hat{q}(t), \hat{r}(t, \cdot)) \mid \varepsilon_{t}\right],
\end{aligned}
$$

for all $t \in[0, \infty)$ a.s.

Then $\hat{u}$ is an optimal control for the problem (4.6).

Proof. Choose an arbitrary $u \in \mathcal{A}_{\varepsilon}$, and consider

$$
J(u)-J(\hat{u})=I_{1},
$$


where

$$
I_{1}=E\left[\int_{0}^{\infty}\{f(t, X(t), Y(t), A(t), u(t))-f(t, \hat{X}(t), \hat{Y}(t), \hat{A}(t), \hat{u}(t))\} d t\right] .
$$

By the definition (4.7) of $H$ and the concavity, we have

$$
\begin{aligned}
I_{1} & \leq E\left[\int _ { 0 } ^ { \infty } \left\{\frac{\partial H}{\partial x}(t)(X(t)-\hat{X}(t))+\frac{\partial H}{\partial y}(t)(Y(t)-\hat{Y}(t))+\frac{\partial H}{\partial a}(t)(A(t)-\hat{A}(t))\right.\right. \\
& +\frac{\partial H}{\partial u}(t)(u(t)-\hat{u}(t))-(b(t)-\hat{b}(t)) \hat{p}(t)-(\sigma(t)-\hat{\sigma}(t)) \hat{q}(t) \\
& \left.\left.-\int_{\mathbb{R}_{0}}(\theta(t, z)-\hat{\theta}(t, z)) \hat{r}(t, z) \nu(d z)\right\} d t\right],
\end{aligned}
$$

where we have used the simplified notation

$$
\frac{\partial H}{\partial x}(t)=\frac{\partial H}{\partial x}(t, \hat{X}(t), \hat{Y}(t), \hat{A}(t), \hat{u}(t), \hat{p}(t), \hat{q}(t), \hat{r}(t, \cdot)),
$$

and similarly for $b$ and $\sigma$.

Applying the Itô formula to $\hat{p}(t)(X(t)-\hat{X}(t))$ we get, by (4.11) and (4.12),

$$
\begin{aligned}
0 & \leq \varlimsup_{T \rightarrow \infty} E[\hat{p}(T)(X(T)-\hat{X}(T))] \\
& =\varlimsup_{T \rightarrow \infty} E\left[\left(\int_{0}^{T}(b(t)-\hat{b}(t)) \hat{p}(t) d t+\int_{0}^{T}(X(t)-\hat{X}(t)) E\left[\hat{\mu}(t) \mid \mathcal{F}_{t}\right] d t\right.\right. \\
& \left.\left.+\int_{0}^{T}(\sigma(t)-\hat{\sigma}(t)) \hat{q}(t) d t+\int_{0}^{T} \int_{\mathbb{R}_{0}}(\theta(t, z)-\hat{\theta}(t, z)) \hat{r}(t, z) \nu(d z) d t\right)\right] \\
& =\varlimsup_{T \rightarrow \infty} E\left[\left(\int_{0}^{T}(b(t)-\hat{b}(t)) \hat{p}(t) d t+\int_{0}^{T}(X(t)-\hat{X}(t)) \hat{\mu}(t) d t\right.\right. \\
& \left.\left.+\int_{0}^{T}(\sigma(t)-\hat{\sigma}(t)) \hat{q}(t) d t+\int_{0}^{T} \int_{\mathbb{R}_{0}}(\theta(t, z)-\hat{\theta}(t, z)) \hat{r}(t, z) \nu(d z) d t\right)\right]
\end{aligned}
$$


Using the definition (4.10) of $\mu$ we see that

$$
\begin{aligned}
\varlimsup_{T \rightarrow \infty} E\left[\left(\int_{0}^{T}(X(t)-\hat{X}(t)) \hat{\mu}(t) d t\right)\right] \\
=\varlimsup_{T \rightarrow \infty} E\left[\left(\int_{\delta}^{T+\delta}(X(t-\delta)-\hat{X}(t-\delta)) \hat{\mu}(t-\delta) d t\right)\right] \\
=\varlimsup_{T \rightarrow \infty} E\left[\left(-\int_{\delta}^{T+\delta} \frac{\partial H}{\partial x}(t-\delta)(X(t-\delta)-\hat{X}(t-\delta)) d t\right.\right. \\
-\int_{\delta}^{T+\delta} \frac{\partial H}{\partial y}(t)(Y(t)-\hat{Y}(t)) d t \\
\left.\left.-\int_{\delta}^{T+\delta}\left(\int_{t-\delta}^{t} \frac{\partial H}{\partial a}(s) e^{-\lambda s} d s\right) e^{\lambda(t-\delta)}(X(t-\delta)-\hat{X}(t-\delta))\right) d t\right] .
\end{aligned}
$$

Using Fubini and substituting $r=t-\delta$, we obtain

$$
\begin{aligned}
\int_{0}^{T} \frac{\partial H}{\partial a}(s)(A(s)-\hat{A}(s)) d s \\
=\int_{0}^{T} \frac{\partial H}{\partial a}(s) \int_{s-\delta}^{s} e^{-\lambda(s-r)}(X(r)-\hat{X}(r)) d r d s \\
=\int_{0}^{T}\left(\int_{r}^{r+\delta} \frac{\partial H}{\partial a}(s) e^{-\lambda s} d s\right) e^{\lambda r}(X(r)-\hat{X}(r)) d r \\
=\int_{0}^{T+\delta}\left(\int_{t-\delta}^{t} \frac{\partial H}{\partial a}(s) e^{-\lambda s} d s\right) e^{\lambda(t-\delta)}(X(t-\delta)-\hat{X}(t-\delta)) d t
\end{aligned}
$$


Combining (4.17), (4.18) and (4.19) we get,

$$
\begin{aligned}
0 \leq & \varlimsup_{T \rightarrow \infty} E[\hat{p}(T)(X(T)-\hat{X}(T))]=E\left[\int_{0}^{\infty}(b(t)-\hat{b}(t)) \hat{p}(t) d t\right. \\
& -\int_{0}^{\infty} \frac{\partial H}{\partial x}(t)(X(t)-\hat{X}(t)) d t-\int_{\delta}^{\infty} \frac{\partial H}{\partial y}(t)(Y(t)-\hat{Y}(t)) d t \\
- & \int_{\delta}^{\infty} \frac{\partial H}{\partial a}(t)(A(t)-\hat{A}(t)) d t+\int_{0}^{\infty}(\sigma(t)-\hat{\sigma}(t)) \hat{q}(t) d t \\
& \left.\left.+\int_{0}^{\infty} \int_{\mathbb{R}_{0}}^{\infty}(\theta(t, z)-\hat{\theta}(t, z)) \hat{r}(t, z) \nu(d z) d t\right)\right]
\end{aligned}
$$

Subtracting and adding $\int_{0}^{\infty} \frac{\partial H}{\partial u}(t)(u(t)-\hat{u}(t)) d t$ in (4.19) we conclude

$$
\begin{aligned}
0 \leq & \varlimsup_{T \rightarrow \infty} E[\hat{p}(T)(X(T)-\hat{X}(T))]=E\left[\int_{0}^{\infty}(b(t)-\hat{b}(t)) \hat{p}(t) d t\right. \\
& -\int_{0}^{\infty} \frac{\partial H}{\partial x}(t)(X(t)-\hat{X}(t)) d t-\int_{\delta}^{\infty} \frac{\partial H}{\partial y}(t)(Y(t)-\hat{Y}(t)) d t \\
& -\int_{\delta}^{\infty} \frac{\partial H}{\partial a}(t)(A(t)-\hat{A}(t)) d t+\int_{0}^{\infty}(\sigma(t)-\hat{\sigma}(t)) \hat{q}(t) d t \\
& +\int_{0}^{\infty} \int_{\mathbb{R}_{0}}^{\infty}(\theta(t, z)-\hat{\theta}(t, z)) \hat{r}(t, z) \nu(d z) d t \\
& \left.\left.-\int_{0}^{\infty} \frac{\partial H}{\partial u}(t)(u(t)-\hat{u}(t)) d t+\int_{0}^{\infty} \frac{\partial H}{\partial u}(t)(u(t)-\hat{u}(t)) d t\right)\right] \\
\leq & -I_{1}+E\left[\int_{0}^{\infty} E\left[\frac{\partial H}{\partial u}(t)(u(t)-\hat{u}(t)) \mid \mathcal{E}_{t}\right] d t\right] .
\end{aligned}
$$

Hence

$$
I_{1} \leq E\left[\int_{0}^{\infty} E\left[\frac{\partial H}{\partial u}(t) \mid \mathcal{E}_{t}\right](u(t)-\hat{u}(t)) d t\right] \leq 0 .
$$

Since $u \in \mathcal{A}_{\mathcal{E}}$ was arbitrary, this proves the Theorem. 


\subsubsection{A second sufficient maximum principle}

We extend the result in Øksendal and Sulem [2007] to infinite horizon with jump diffusions.

Consider again the system

$$
\begin{cases}d X(t)=b(t, X(t), Y(t), A(t), u(t)) d t & \\ +\sigma(t, X(t), Y(t), A(t), u(t)) d B(t) & \\ +\int_{\mathbb{R}_{0}} \theta(t, X(t), Y(t), A(t), u(t), z) \tilde{N}(d t, d z) ; & t \in[0, \infty), \\ X(t)=X_{0}(t) ; & t \in[-\delta, 0], \\ Y(t)=X(t-\delta) ; & t \in[0, \infty), \\ A(t)=\int_{t-\delta}^{t} e^{-\lambda(t-r)} X(r) d r ; & t \in[0, \infty) .\end{cases}
$$

We now give an Itô formula which is proved in Elsanosi et al. [2000] without jumps. Adding the jump parts is just an easy observation.

Lemma 4.3. The Itô formula for delayed system

Consider a function

$$
G(t)=F(t, X(t), A(t)),
$$

where $F$ is a function in $C^{1,2,1}\left(\mathbb{R}^{3}\right)$. Note that

$$
A(t)=\int_{-\delta}^{0} e^{\lambda s} X(t+s) d s
$$

Then

$$
\begin{aligned}
d G(t)= & (L F)(t, X(t), Y(t), A(t), u(t)) d t \\
+ & \sigma(t, X(t), Y(t), A(t), u(t)) \frac{\partial F}{\partial x}(t, X(t), A(t)) d B(t) \\
& +\int_{\mathbb{R}_{0}}\left\{F\left(t, X\left(t^{-}\right)+\theta(t, X(t), Y(t), A(t), u(t), z), A\left(t^{-}\right)\right)\right. \\
& -F\left(t, X\left(t^{-}\right), A\left(t^{-}\right)\right) \\
& \left.-\frac{\partial F}{\partial x}\left(t, X\left(t^{-}\right), A\left(t^{-}\right)\right) \theta(t, X(t), Y(t), A(t), u(t), z)\right\} \nu(d z) d t \\
+ & \int_{\mathbb{R}_{0}}\left\{F\left(t, X\left(t^{-}\right)+\theta(t, X(t), Y(t), A(t), u(t), z), A\left(t^{-}\right)\right)\right. \\
& \left.-F\left(t, X\left(t^{-}\right), A\left(t^{-}\right)\right)\right\} \tilde{N}(d t, d z) \\
+ & {\left[X(t)-\lambda A(t)-e^{-\lambda \delta} Y(t)\right] \frac{\partial F}{\partial a}(t, X(t), A(t)) d t, }
\end{aligned}
$$

where

$$
L F=L F(t, x, y, a, u)=\frac{\partial F}{\partial t}+b(t, x, y, a, u) \frac{\partial F}{\partial x}+\frac{1}{2} \sigma^{2}(t, x, y, a, u) \frac{\partial^{2} F}{\partial x^{2}}
$$


In particular, note that

$$
d A(t)=X(t)-\lambda A(t)-e^{-\lambda \delta} Y(t) ; t \geq 0
$$

Now, define the Hamiltonian, $H^{\prime}: \mathbb{R}_{+} \times \mathbb{R} \times \mathbb{R} \times \mathbb{R} \times \mathcal{U} \times \mathbb{R}^{3} \times \mathbb{R}^{3} \times \Re \rightarrow \mathbb{R}$ as

$$
\begin{aligned}
& H^{\prime}(t, x, y, a, u, p, q, r(\cdot)) \\
& =f(t, x, y, a, u)+b(t, x, y, a, u) p_{1}+\left(x-\lambda a-e^{-\lambda \delta} y\right) p_{3} \\
& +\sigma(t, x, y, a, u) q_{1}+\int_{\mathbb{R}_{0}} \theta(t, x, y, a, u, z) r(z) \nu(d z),
\end{aligned}
$$

where $p=\left(p_{1}, p_{2}, p_{3}\right)^{T} \in \mathbb{R}^{3}$ and $q=\left(q_{1}, q_{2}, q_{3}\right) \in \mathbb{R}^{3}$. For each $u \in \mathcal{A}_{\varepsilon}$ the associated adjoint equations are the following backward stochastic differential equations in the unknown $\mathcal{F}_{t}$-adapted processes $(p(t), q(t), r(t, \cdot))$ given by

$$
\begin{aligned}
d p_{1}(t) & =-\frac{\partial H^{\prime}}{\partial x}(t, X(t), Y(t), A(t), u(t), p(t), q(t), r(t, \cdot)) d t+q_{1}(t) d B(t) \\
& +\int_{\mathbb{R}_{0}} r(t, z) \tilde{N}(d t, d z), \\
d p_{2}(t) & =-\frac{\partial H^{\prime}}{\partial y}(t, X(t), Y(t), A(t), u(t), p(t), q(t), r(t, \cdot)) d t \\
d p_{3}(t) & =-\frac{\partial H^{\prime}}{\partial a}(t, X(t), Y(t), A(t), u(t), p(t), q(t), r(t, \cdot)) d t+q_{3}(t) d B(t),
\end{aligned}
$$

Theorem 4.4 (A second infinite horizon maximum principle for delay equations). Suppose $\hat{u} \in \mathcal{A}_{\varepsilon}$ and let $(\hat{X}(t), \hat{Y}(t), \hat{A}(t))$ and $(\hat{p}(t), \hat{q}(t), \hat{r}(t, \cdot))$ be corresponding solutions of (4.25)(4.27), respectively. Suppose that

$$
\left.(x, y, a, u) \mapsto H^{\prime}(t, x, y, a, u, \hat{p}(t), \hat{q}(t), \hat{r}(t, \cdot))\right)
$$

is concave for all $t \geq 0$ a.s. and

$$
\begin{aligned}
& E\left[H^{\prime}(t, \hat{X}(t), \hat{Y}(t), \hat{A}(t), \hat{u}(t), \hat{p}(t), \hat{q}(t), \hat{r}(t, \cdot)) \mid \mathcal{E}_{t}\right] \\
& =\max _{u \in \mathcal{U}} E\left[H^{\prime}(t, \hat{X}(t), \hat{Y}(t), \hat{A}(t), u, \hat{p}(t), \hat{q}(t), \hat{r}(t, \cdot)) \mid \mathcal{E}_{t}\right] .
\end{aligned}
$$

Further, assume that

$$
\varlimsup_{T \rightarrow \infty} E\left[\hat{p}_{1}(T)(X(T)-\hat{X}(T))+\hat{p}_{3}(T)(A(T)-\hat{A}(T))\right] \geq 0 .
$$

In addition assume that

$$
\hat{p}_{2}(t)=0,
$$

for all $t$. Then $\hat{u}$ is an optimal control for the control problem (4.6). 
Proof. To simplify notation we put

$$
\zeta(t)=(X(t), Y(t), A(t))
$$

and

$$
\hat{\zeta}(t)=(\hat{X}(t), \hat{Y}(t), \hat{A}(t))
$$

Let

$$
I:=J(\hat{u})-J(u)=E\left[\int_{0}^{\infty}(f(t, \hat{\zeta}(t), \hat{u}(t))-f(t, \zeta(t), u(t))) d t\right]
$$

Then we have that

$$
\begin{aligned}
I & =\varlimsup_{T \rightarrow \infty} E\left[\int_{0}^{T}\left(H^{\prime}(t, \hat{\zeta}(t), \hat{u}(t), \hat{p}(t), \hat{q}(t), \hat{r}(t, \cdot))-H^{\prime}(t, \zeta(t), u(t), \hat{p}(t), \hat{q}(t), \hat{r}(t, \cdot)) d t\right]\right. \\
& -E\left[\int_{0}^{T}\left(b(t, \hat{\zeta}(t), \hat{u}(t))-b(t, \zeta(t), u(t)) \hat{p}_{1}(t) d t\right]\right. \\
& -E\left[\int_{0}^{T}\left\{\left(\hat{X}(t)-\lambda \hat{A}(t)-e^{-\lambda \delta} \hat{Y}(t)\right)-\left(X(t)-\lambda A(t)-e^{-\lambda \delta} Y(t)\right)\right\} \hat{p}_{3}(t) d t\right] \\
& -E\left[\int_{0}^{T}\{\sigma(t, \hat{\zeta}(t), \hat{u}(t))-\sigma(t, \zeta(t), u(t))\} \hat{q}_{1}(t) d t\right] \\
& -E\left[\int_{0}^{T} \int_{\mathbb{R}_{0}}(\theta(t, \hat{\zeta}(t), \hat{u}(t), z)-\theta(t, \zeta, u(t), z)) \hat{r}(t, z) \nu(d z) d t\right] \\
& =: I_{1}+I_{2}+I_{3}+I_{4}+I_{5} .
\end{aligned}
$$

Since $(\zeta, u) \rightarrow H^{\prime}(t, \zeta, u, \hat{p}(t), \hat{q}(t), \hat{r}(t, \cdot))$ is concave, we have by (4.28) that

$$
\begin{aligned}
& H^{\prime}(t, \zeta, u, \hat{p}(t), \hat{q}(t), \hat{r}(t, \cdot))-H^{\prime}(t, \hat{\zeta}, \hat{u}, \hat{p}(t), \hat{q}(t), \hat{r}(t, \cdot)) \\
& \leq \nabla_{\zeta} H^{\prime}(t, \hat{\zeta}, \hat{u}, \hat{p}(t), \hat{q}(t), \hat{r}(t, \cdot)) \cdot(\zeta-\hat{\zeta})+\frac{\partial H^{\prime}}{\partial u}(t, \hat{\zeta}, \hat{u}, \hat{p}(t), \hat{q}(t), \hat{r}(t, \cdot)) \cdot(u-\hat{u}) \\
& \leq \nabla_{\zeta} H^{\prime}(t, \hat{\zeta}, \hat{u}, \hat{p}(t), \hat{q}(t), \hat{r}(t, \cdot)) \cdot(\zeta-\hat{\zeta})
\end{aligned}
$$

where $\nabla_{\zeta} H^{\prime}=\left(\frac{\partial H^{\prime}}{\partial x}, \frac{\partial H^{\prime}}{\partial y}, \frac{\partial H^{\prime}}{\partial a}\right)$. From this we get that

$$
\begin{aligned}
I_{1} & \geq \varlimsup_{T \rightarrow \infty} E\left[\int_{0}^{T}-\nabla_{\zeta} H^{\prime}(t, \hat{\zeta}(t), \hat{u}(t), \hat{p}(t), \hat{q}(t), \hat{r}(t, \cdot)) \cdot(\zeta(t)-\hat{\zeta}(t)) d t\right] \\
& =\varlimsup_{T \rightarrow \infty} E\left[\int_{0}^{T}(\zeta(t)-\hat{\zeta}(t)) d \hat{p}(t)\right] \\
& =E\left[\int_{0}^{\infty}(X(t)-\hat{X}(t)) d \hat{p}_{1}(t)+\int_{0}^{\infty}(A(t)-\hat{A}(t)) d \hat{p}_{3}(t)\right] .
\end{aligned}
$$


From (4.25), (4.26) and (4.27) we get that

$$
\begin{aligned}
0 & \geq-\varlimsup_{T \rightarrow \infty} E\left[\hat{p}_{1}(T)(X(T)-\hat{X}(T))+\hat{p}_{3}(T)(A(T)-\hat{A}(T))\right] \\
& =-\varlimsup_{T \rightarrow \infty} E\left[\int_{0}^{T}(X(t)-\hat{X}(t)) d \hat{p}_{1}(t)+\int_{0}^{T} \hat{p}_{1}(t) d(X(t)-\hat{X}(t))\right. \\
& +\int_{0}^{T}[\sigma(t, \zeta(t), u(t))-\sigma(t, \hat{\zeta}(t), \hat{u}(t))] \hat{q}_{1}(t) d t \\
& +\int_{0}^{T} \int_{\mathbb{R}_{0}}(\theta(t, \zeta(t), u(t), z)-\theta(t, \hat{\zeta}, \hat{u}(t), z)) \hat{r}(t, z) \nu(d z) d t \\
& \left.+\int_{0}^{T}(A(t)-\hat{A}(t)) d \hat{p}_{3}(t)+\int_{0}^{T} \hat{p}_{3}(t) d(A(t)-\hat{A}(t))\right]
\end{aligned}
$$

Combining this with (4.30) and (4.31) and using (4.23), we have that

$$
-I=I_{1}+I_{2}+I_{3}+I_{4}+I_{5} \leq 0 .
$$

Hence $J(\hat{u})-J(u)=I \geq 0$, and $\hat{u}$ is an optimal control for our problem.

Example 4.5 (A non-delay infinite horizon example). Let us first consider a non-delay example. Assume we are given the performance functional

$$
J(u)=E\left[\int_{0}^{\infty} e^{-\rho t} \frac{1}{\gamma} u^{\gamma}(t) d t\right],
$$

and the state equation

$$
\left\{\begin{aligned}
d X(t) & =[X(t) \mu-u(t)] d t \\
& +\sigma(t, X(t), u(t)) d B(t) ; t \geq 0 \\
X(0) & =X_{0}>0
\end{aligned}\right.
$$

where $X_{0}>0, \gamma \in(0,1), \rho>0$ and $\mu \in \mathbb{R}$ are given constants. We assume that

$$
\mu \gamma<\rho
$$

Here $u(t) \geq 0$ is our control. It can be interpreted as the consumption rate from a cash flow $X(t)$. The performance $J(u)$ is the total expected discounted utility of the consumption. For $u$ to be admissible we require that $E[X(t)] \geq 0$ for all $t \geq 0$.

In this case the Hamiltonian (4.24) takes the form

$$
\begin{aligned}
H^{\prime}(t, x, u, p, q) & =e^{-\rho t} \frac{1}{\gamma} u^{\gamma}+[x \mu-u] p \\
& +\sigma(t, x, u) q,
\end{aligned}
$$


so that we get the partial derivative

$$
\frac{\partial H^{\prime}}{\partial u}(t, u, x, p, q)=e^{-\rho t} u^{\gamma-1}-p+\frac{\partial \sigma}{\partial u} q
$$

Therefore, if $\frac{\partial H^{\prime}}{\partial u}=0$ we get

$$
p(t)=e^{-\rho t} u^{\gamma-1}(t)+\frac{\partial \sigma}{\partial u}(t, X(t), u(t)) q(t) .
$$

We now see that the adjoint equation is given by:

$$
d p(t)=-\left[\mu p(t)+\frac{\partial \sigma}{\partial x}(t, X(t), u(t)) q(t)\right] d t+q(t) d B(t)
$$

Now assume that

$$
\sigma(t, x, u)=\sigma_{0}(t) x
$$

for some bounded adapted process $\sigma_{0}(t)$. Let us try to choose $q=0$. Then

$$
d p(t)=-\mu p(t) d t
$$

which gives

$$
p(t)=p(0) e^{-\mu t},
$$

for some constant $p(0)$. Hence, by (4.37)

$$
\hat{u}(t)=p^{\frac{1}{\gamma-1}}(0) e^{\frac{(\mu-\rho) t}{1-\gamma}},
$$

for all $t>0$. Inserting $\hat{u}(t)$ into the dynamics of $\hat{X}(t)$, we get that

$$
d \hat{X}(t)=\left[\mu \hat{X}(t)-p^{\frac{1}{\gamma-1}}(0) e^{\frac{1}{\gamma-1}(\rho t-\mu t)}\right] d t+\sigma_{0}(t) \hat{X}(t) d B(t) .
$$

So

$$
\hat{X}(t)=\left[\hat{X}(0) \Gamma(t)-p^{\frac{1}{\gamma-1}}(0) \int_{0}^{t} \frac{\Gamma(t)}{\Gamma(s)} \exp \left(\frac{(\mu-\rho) s}{1-\gamma}\right) d s\right] .
$$

where

$$
\Gamma(t)=\exp \left(\int_{0}^{t} \sigma_{0}(s) d B(s)+\mu t-\frac{1}{2} \int_{0}^{t} \sigma_{0}^{2}(s) d s\right) .
$$

Hence

$$
E[\hat{X}(t)]=e^{\mu t}\left[\hat{X}(0)-p^{\frac{1}{\gamma-1}}(0) \int_{0}^{t} \exp \left(\frac{(\mu \gamma-\rho) s}{1-\gamma}\right) d s\right]
$$


Therefore, to ensure that $E[\hat{X}(t)]$ is non-negative, we get the optimal $\hat{p}(0)$ as

$$
\hat{p}(0)=\left[\frac{\hat{X}(0)}{\int_{0}^{\infty} \exp \left(\frac{(\mu \gamma-\rho) s}{1-\gamma}\right) d s}\right]^{\gamma-1} .
$$

We now see that $\varlimsup_{T \rightarrow \infty} E[\hat{p}(T) \hat{X}(T)]=0$, so that we have

$$
\varlimsup_{T \rightarrow \infty} E[\hat{p}(T)(X(T)-\hat{X}(T))] \geq 0 .
$$

This tells us that $\hat{u}$ with $p(0)=\hat{p}(0)$ given by (4.42) the control $\hat{u}$ given by (4.39) is indeed an optimal control.

Example 4.6 (An infinite horizon example with delay). Now let us consider a case where we have delay. This is an infinite horizon version of Example 1 in Øksendal and Sulem [2007]. Let

$$
J(u)=E\left[\int_{0}^{\infty} e^{-\rho t} \frac{1}{\gamma} u(t)^{\gamma} d t\right]
$$

and define

$$
\begin{cases}d X(t) & =d X^{(u)}(t)=[X(t) \mu+Y(t) \beta+\alpha A(t)-u(t)] d t \\ & +\sigma(t, X(t), Y(t), A(t), u(t)) d B(t) ; t \geq 0, \\ X(t) & =X_{0}(t)>0 ; t \in[-\delta, 0] .\end{cases}
$$

We want to find a consumption rate $u^{*}(t)$ such that

$$
J\left(u^{*}\right)=\sup \left\{J(u) ; E\left[X^{(u)}(t)\right] \geq 0 \text { for all } t \geq 0\right\} .
$$

Here $\gamma \in(0,1), \rho, \delta \geq 0$ and $\beta \in \mathbb{R}$ are given constants.

In this case the Hamiltonian (4.24) takes the form

$$
\begin{aligned}
H^{\prime}(t, x, u, y, a, p, q) & =e^{-\rho t} \frac{1}{\gamma} u^{\gamma}+[x \mu+\beta y+\alpha a-u] p_{1} \\
& +\left[x-\lambda a-e^{-\lambda \delta} y\right] p_{3}+\sigma(t, x, y, a, u) q_{1},
\end{aligned}
$$

so that we get the partial derivative

$$
\frac{\partial H^{\prime}}{\partial u}(t, x, u, y, a, p, q)=e^{-\rho t} u^{\gamma-1}-p_{1}+\frac{\partial \sigma}{\partial u} q_{1}
$$

This, together with the maximality condition, gives that

$$
p_{1}(t)=e^{-\rho t} u(t)^{\gamma-1}+\frac{\partial \sigma}{\partial u} q_{1}
$$


We now see that the adjoint equations are given by:

$$
\begin{aligned}
& d p_{1}(t)=-\left[\mu p_{1}(t)+p_{3}(t)+\frac{\partial \sigma}{\partial x} q_{1}(t)\right] d t+q_{1}(t) d B(t) \\
& d p_{2}(t)=-\left[\beta p_{1}(t)-e^{-\lambda \delta} p_{3}(t)+\frac{\partial \sigma}{\partial y} q_{1}(t)\right] d t \\
& d p_{3}(t)=-\left[\alpha p_{1}(t)-\lambda p_{3}(t)+\frac{\partial \sigma}{\partial a} q_{1}(t)\right] d t+q_{3}(t) d B(t)
\end{aligned}
$$

Since the coefficents in front of $p_{1}$ and $p_{3}$ are deterministic we can choose $q_{1}=q_{3}=0$. Since we want $p_{2}(t)=0$, we then get

$$
p_{1}(t)=\frac{e^{-\lambda \delta}}{\beta} p_{3}(t)
$$

which gives us that

$$
\begin{aligned}
& d p_{1}(t)=-\left[\mu p_{1}(t)+\beta e^{\lambda \delta} p_{1}(t)\right] d t \\
& d p_{3}(t)=-\left[\frac{\alpha}{\beta} e^{-\lambda \delta} p_{3}(t)-\lambda p_{3}(t)\right] d t
\end{aligned}
$$

and

$$
u(t)=e^{\frac{\rho t}{\gamma-1}} p_{1}^{\frac{1}{\gamma-1}}(t) .
$$

Hence, to ensure that

$$
p_{1}(t)=\frac{e^{-\lambda \delta}}{\beta} p_{3}(t)
$$

we need that

$$
\alpha=\beta e^{\lambda \delta}\left(\mu+\lambda+\beta e^{\lambda \delta}\right) .
$$

So

$$
p_{1}(t)=p_{1}(0) e^{-\left(\mu+\beta e^{\lambda \delta}\right) t},
$$

for some constant $p_{1}(0)$. Hence by (4.46) we get

$$
u(t)=u_{p_{1}(0)}=p_{1}(0)^{\frac{1}{\gamma-1}} \exp \left(\frac{\left(\mu+\beta e^{\lambda \delta}-\rho\right) t}{1-\gamma}\right),
$$

for all $t>0$ and some $p_{1}(0)$. Now assume that

$$
\alpha=0 \text {, i.e. } \lambda+\beta e^{\lambda \delta}=-\mu,
$$

and that

$$
\sigma(t, X(t), Y(t), A(t), u(t))=\kappa A(t)(\kappa \text { constant })
$$

Then (3.37) gets the form

$$
\left\{\begin{array}{l}
d X(t)=[\mu X(t)+\beta Y(t)-u(t)] d t+\kappa A(t) d B(t) ; t \geq 0 \\
X(t)=X_{0}(t) ; t \in[-\delta, 0]
\end{array}\right.
$$


and

$$
p_{1}(t)=p_{1}(0) e^{\lambda t}
$$

Let $\theta$ be the unique solution of the equation

$$
\mu+\theta+|\beta| e^{\theta \delta}=0
$$

Then by Corollary 4.1 in Mohammed and Scheutzow [1997] the top a.s. Lyapunov exponent $\lambda_{1}$ of the solution $X^{(0)}(t)$ of the stochastic delay equation (4.53) corresponding to $u=0$ satisfies the inequality

$$
\lambda_{1} \leq-\theta+\frac{\kappa^{2}}{2|\beta|} e^{|\theta| \delta}
$$

Therefore we see that

$$
\begin{aligned}
& \lim _{T \rightarrow \infty} \hat{p}_{1}(T) \hat{X}(T) \leq \lim _{T \rightarrow \infty} \hat{p}_{1}(T) \hat{X}^{(0)}(T) \\
& \leq \text { const. } \lim _{T \rightarrow \infty} \exp \left(-\left(-\lambda+\theta-\frac{\kappa^{2}}{2|\beta|} e^{|\theta| \delta}\right) T\right)=0
\end{aligned}
$$

if

$$
\lambda+\frac{\kappa^{2}}{2|\beta|} e^{|\theta| \delta}<\theta .
$$

By (4.47) condition (4.57) also implies that

$$
\lim _{T \rightarrow \infty} \hat{p}_{3}(T) \hat{A}(T)=0
$$

We conclude that (4.29) holds. It remains to determine the optimal value of $\hat{p}_{1}(0)$. To maximize the expected utility of the consumption (4.43), we choose $\hat{p}_{1}(0)$ as big as possible under the constraint that $E[\hat{X}(t)] \geq 0$ for all $t \geq 0$. Hence we put

$$
\hat{p}_{1}(0)=\sup \left\{p_{1}(0) ; E\left[X^{(\hat{u})}(t)\right] \geq 0 \text { for all } t \geq 0\right\},
$$

where

$$
\left\{\begin{aligned}
& d X^{(\hat{u})}(t)=\left[X^{(\hat{u})}(t) \mu+Y^{(\hat{u})}(t) \beta-p_{1}^{\frac{1}{\gamma-1}}(0) \exp \left(\frac{-(\lambda+\rho) t}{1-\gamma}\right)\right] d t \\
&+\kappa A^{(\hat{u})}(t) d B(t) ; t \geq 0, \\
& X^{(\hat{u})}(t) \quad=X_{0}(t)>0 ; t \in[-\delta, 0] .
\end{aligned}\right.
$$

In this case, however, in lack of a solution formula for $E\left[X^{(u)}(t)\right]$, we are not able to find an explicit expression for $\hat{p}_{1}(0)$, as we could in the above example. We conclude that our candidate 
for the optimal control is given by

$$
\hat{u}(t)=\hat{p}_{1}^{\frac{1}{\gamma-1}}(0) \exp \left(\frac{-(\lambda+\rho) t}{1-\gamma}\right) .
$$

\subsection{A necessary maximum principle}

In addition to the assumptions in Sections 4.2 and 4.3.1, we now assume the following.

$\left(A_{1}\right)$ For all $u \in \mathcal{A}_{\varepsilon}$ and all $\beta \in \mathcal{A}_{\varepsilon}$ bounded, there exists $\epsilon>0$ such that

$$
u+s \beta \in \mathcal{A}_{\varepsilon} \quad \text { for all } s \in(-\epsilon, \epsilon) .
$$

$\left(A_{2}\right)$ For all $t_{0}, h$ such that $0 \leq t_{0}<t_{0}+h \leq T$ and all bounded $\varepsilon_{t_{0}}$-measurable random variables $\alpha$, the control process $\beta(t)$ defined by

$$
\beta(t)=\alpha 1_{\left[t_{0}, t_{0}+h\right]}(t)
$$

belongs to $\mathcal{A}_{\mathcal{E}}$.

$\left(A_{3}\right)$ The derivative process

$$
\xi(t):=\left.\frac{d}{d s} X^{u+s \beta}(t)\right|_{s=0}
$$

exists and belongs to $L^{2}(m \times P)$, where $m$ denotes the Lebesgue measure on $\mathbb{R}$.

It follows from (4.3) that

$$
\begin{aligned}
& d \xi(t)=\left\{\frac{\partial b}{\partial x}(t) \xi(t)+\frac{\partial b}{\partial y}(t) \xi(t-\delta)+\frac{\partial b}{\partial a}(t) \int_{t-\delta}^{t} e^{-\lambda(t-r)} \xi(r) d r+\frac{\partial b}{\partial u}(t) \beta(t)\right\} d t \\
& +\left\{\frac{\partial \sigma}{\partial x}(t) \xi(t)+\frac{\partial \sigma}{\partial y}(t) \xi(t-\delta)+\frac{\partial \sigma}{\partial a}(t) \int_{t-\delta}^{t} e^{-\lambda(t-r)} \xi(r) d r+\frac{\partial \sigma}{\partial u}(t) \beta(t)\right\} d B(t) \\
& +\int_{\mathbb{R}_{0}}\left\{\frac{\partial \theta}{\partial x}(t, z) \xi(t)+\frac{\partial \theta}{\partial y}(t, z) \xi(t-\delta)+\frac{\partial \theta}{\partial a}(t, z) \int_{t-\delta}^{t} e^{-\lambda(t-r)} \xi(r) d r+\frac{\partial \theta}{\partial u}(t, z) \beta(t)\right\} \tilde{N}(d t, d z),
\end{aligned}
$$

where, for simplicity of notation, we define

$$
\frac{\partial}{\partial x} b(t):=\frac{\partial}{\partial x} b(t, X(t), X(t-\delta), A(t), u(t)),
$$

and use that

$$
\left.\frac{d}{d s} Y^{u+s \beta}(t)\right|_{s=0}=\left.\frac{d}{d s} X^{u+s \beta}(t-\delta)\right|_{s=0}=\xi(t-\delta),
$$

and 


$$
\begin{aligned}
\left.\frac{d}{d s} A^{u+s \beta}(t)\right|_{s=0} & =\left.\frac{d}{d s}\left(\int_{t-\delta}^{t} e^{-\lambda(t-r)} X^{u+s \beta}(r) d r\right)\right|_{s=0} \\
& =\left.\left(\int_{t-\delta}^{t} e^{-\lambda(t-r)} \frac{d}{d s} X^{u+s \beta}(r) d r\right)\right|_{s=0} \\
& =\int_{t-\delta}^{t} e^{-\lambda(t-r)} \xi(r) d r .
\end{aligned}
$$

Note that

$$
\xi(t)=0 \text { for } t \in[-\delta, 0]
$$

Theorem 4.7 (Necessary maximum principle). Suppose that $\hat{u} \in \mathcal{A}_{\mathcal{E}}$ with corresponding solutions $\hat{X}(t)$ of (4.3)-(4.4) and $\hat{p}(t), \hat{q}(t)$, and $\hat{r}(t, \cdot)$ of (4.8)-(4.9), and corresponding derivative process $\hat{\xi}(t)$ given by (4.62). Assume that for all $u \in \mathcal{A}_{\varepsilon}$ with corresponding $(X(t), p(t), q(t), r(t, \cdot))$ the following hold:

$$
\begin{aligned}
& E\left[\int _ { 0 } ^ { T } \hat { p } ^ { 2 } ( t ) \left\{\left(\frac{\partial \sigma}{\partial x}\right)^{2}(t) \hat{\xi}^{2}(t)+\left(\frac{\partial \sigma}{\partial y}\right)^{2}(t) \hat{\xi}^{2}(t-\delta)+\left(\frac{\partial \sigma}{\partial a}\right)^{2}(t)\left(\int_{t-\delta}^{t} e^{-\lambda(t-r)} \hat{\xi}(r) d r\right)^{2}\right.\right. \\
& +\left(\frac{\partial \sigma}{\partial u}\right)^{2}(t)+\int_{\mathbb{R}_{0}}\left\{\left(\frac{\partial \theta}{\partial x}\right)^{2}(t, z) \hat{\xi}^{2}(t)+\left(\frac{\partial \theta}{\partial y}\right)^{2}(t, z) \hat{\xi}^{2}(t-\delta)\right. \\
& \left.\left.+\left(\frac{\partial \theta}{\partial a}\right)^{2}(t, z)\left(\int_{t-\delta}^{t} e^{-\lambda(t-r)} \hat{\xi}(r) d r\right)^{2}+\left(\frac{\partial \theta}{\partial u}\right)^{2}(t, z)\right\} \nu(d z)\right\} d t \\
& \left.+\int_{0}^{T} \hat{\xi}^{2}(t)\left\{\hat{q}^{2}(t)+\int_{\mathbb{R}_{0}} \hat{r}^{2}(t, z) \nu(d z)\right\} d t\right]<\infty, \text { for all } T<\infty
\end{aligned}
$$

and

$$
\lim _{T \rightarrow \infty} E[\hat{p}(T) \hat{\xi}(T)]=0 .
$$

Then the following assertions are equivalent.

(i) For all bounded $\beta \in \mathcal{A}_{\varepsilon}$,

$$
\left.\frac{d}{d s} J(\hat{u}+s \beta)\right|_{s=0}=0 .
$$

(ii) For all $t \in[0, \infty)$,

$$
E\left[\frac{\partial H}{\partial u}(t, \hat{X}(t), \hat{Y}(t), \hat{A}(t), u, \hat{p}(t), \hat{q}(t), \hat{r}(t, \cdot)) \mid \mathcal{E}_{t}\right]_{u=\hat{u}(t)}=0 \text { a.s. }
$$


Proof. Suppose that assertion $(i)$ holds. Then

$$
\begin{aligned}
0 & =\left.\frac{d}{d s} J(\hat{u}+s \beta)\right|_{s=0} \\
& =\frac{d}{d s} E\left[\left.\int_{0}^{\infty} f\left(t, X^{\hat{u}+s \beta}(t), Y^{\hat{u}+s \beta}(t), A^{\hat{u}+s \beta}(t), \hat{u}(t)+s \beta(t) d t\right]\right|_{s=0}\right. \\
& =E\left[\int_{0}^{\infty}\left\{\frac{\partial f}{\partial x}(t) \xi(t)+\frac{\partial f}{\partial y}(t) \xi(t-\delta)+\frac{\partial f}{\partial a}(t) \int_{t-\delta}^{t} e^{-\lambda(t-r)} \xi(r) d r+\frac{\partial f}{\partial u}(t) \beta(t)\right\} d t\right] .
\end{aligned}
$$

We know by the definition of $H$ that

$$
\frac{\partial f}{\partial x}(t)=\frac{\partial H}{\partial x}(t)-\frac{\partial b}{\partial x}(t) p(t)-\frac{\partial \sigma}{\partial x}(t) q(t)-\int_{\mathbb{R}_{0}} \frac{\partial \theta}{\partial x}(t, z) r(t, z) \nu(d z)
$$

and the same for $\frac{\partial f}{\partial y}(t), \frac{\partial f}{\partial a}(t)$ and $\frac{\partial f}{\partial u}(t)$.

Applying the Itô formula to $\hat{p}(t) \hat{\xi}(t)$, we obtain by (4.7) and (4.67)

$$
\begin{aligned}
0 & =\lim _{T \rightarrow \infty} E[\hat{p}(T) \xi(T)] \\
& =E\left[\int_{0}^{\infty} \hat{p}(t)\left\{\frac{\partial b}{\partial x}(t) \xi(t)+\frac{\partial b}{\partial y}(t) \xi(t-\delta)+\frac{\partial b}{\partial a}(t) \int_{t-\delta}^{t} e^{-\lambda(t-r)} \xi(r) d r+\frac{\partial b}{\partial u}(t) \beta(t)\right\} d t\right. \\
& +\int_{0}^{\infty} \xi(t) E\left(\mu(t) \mid \mathcal{F}_{t}\right) d t \\
& +\int_{0}^{\infty} q(t)\left\{\frac{\partial \sigma}{\partial x}(t) \xi(t)+\frac{\partial \sigma}{\partial y}(t) \xi(t-\delta)+\frac{\partial \sigma}{\partial a}(t) \int_{t-\delta}^{t} e^{-\lambda(t-r)} \xi(r) d r+\frac{\partial \sigma}{\partial u}(t) \beta(t)\right\} d t \\
& +\int_{0}^{\infty} \int_{\mathbb{R}_{0}} r(t, z)\left\{\frac{\partial \theta}{\partial x}(t, z) \xi(t)+\frac{\partial \theta}{\partial y}(t, z) \xi(t-\delta)+\frac{\partial \theta}{\partial a}(t, z) \int_{t-\delta}^{t} e^{-\lambda(t-r)} \xi(r) d r\right. \\
& \left.\left.+\frac{\partial \theta}{\partial u}(t, z) \beta(t)\right\} \nu(d z) d t\right] \\
& =-\frac{d}{d s} J(\hat{u}+s \beta) \mid s=0+E\left(\int_{0}^{\infty} \frac{\partial H}{\partial u}(t) \beta(t) d t\right) .
\end{aligned}
$$

Therefore

$$
E\left(\int_{0}^{\infty} \frac{\partial H}{\partial u}(t) \beta(t) d t\right)=\left.\frac{d}{d s} J(\hat{u}+s \beta)\right|_{s=0}
$$


Now apply this to

$$
\beta(t)=\alpha 1_{\left[t_{0}, t_{0}+h\right]}(t),
$$

where $\alpha$ is bounded and $\mathcal{E}_{t_{0}}$-measurable, $0 \leq t_{0}<t_{o}+h \leq T$. Then if (i) holds we get

$$
E\left(\int_{t_{0}}^{t_{0}+h} \frac{\partial H}{\partial u}(t) d t \alpha\right)=0
$$

Differentiating with respect to $h$ at 0 , we have

$$
E\left(\frac{\partial H}{\partial u}\left(t_{0}\right) \alpha\right)=0 .
$$

This holds for all $\varepsilon_{t_{0}}$-measurable $\alpha$ and hence we obtain that

$$
E\left(\frac{\partial H}{\partial u}\left(t_{0}\right) \mid \mathcal{E}_{t_{0}}\right)=0
$$

This proves that assertion $(i)$ implies $(i i)$.

To complete the proof, we need to prove the converse implication; which is obtained since every bounded $\beta \in \mathcal{A}_{\varepsilon}$ can be approximated by linear combinations of controls $\beta$ of the form (4.61).

\subsection{Existence and uniqueness of the time-advanced BSDEs on infinite horizon}

The main result in this section refers to the existence and uniqueness for (4.9)-(4.10) where the coefficients satisfy a Lipschitz condition.

Given a positive constant $\delta$, denote by $D([0, \delta], \mathbb{R})$ the space of all càdlàg paths from $[0, \delta]$ into $\mathbb{R}$. For a path $X(\cdot): \mathbb{R}_{+} \rightarrow \mathbb{R}, X_{t}$ will denote the function defined by $X_{t}(s)=X(t+s)$ for $s \in[0, \delta]$. Let $\mathcal{H}=L^{2}(\nu)$ be the set of all functions $r: \mathbb{R}_{0} \rightarrow \mathbb{R}$ such that $\int_{\mathbb{R}_{0}} r^{2}(z) \nu(d z)<\infty$. Consider the $L^{2}$ space $V_{1}:=L^{2}([0, \delta] \rightarrow \mathbb{R} ; d s)$ and $V_{2}:=L^{2}([0, \delta] \rightarrow \mathcal{H} ; d s)$. Let

$$
F: \mathbb{R}_{+} \times \mathbb{R} \times \mathbb{R} \times V_{1} \times \mathbb{R} \times \mathbb{R} \times V_{1} \times \mathcal{H} \times \mathcal{H} \times V_{2} \times \Omega \rightarrow \mathbb{R}
$$

be a function satisfying the following Lipschitz condition: There exists a positive constant $C$ such that

$$
\begin{aligned}
& \left|F\left(t, p_{1}, p_{2}, p, q_{1}, q_{2}, q, r_{1}, r_{2}, r, \omega\right)-F\left(t, \bar{p}_{1}, \bar{p}_{2}, \bar{p}, \bar{q}_{1}, \bar{q}_{2}, \bar{q}, \bar{r}_{1}, \bar{r}_{2}, \bar{r}, \omega\right)\right| \\
& \leq C\left(\left|p_{1}-\bar{p}_{1}\right|+\left|p_{2}-\bar{p}_{2}\right|+|p-\bar{p}|_{V_{1}}+\left|q_{1}-\bar{q}_{1}\right|+\left|q_{2}-\bar{q}_{2}\right|+|q-\bar{q}|_{V_{1}}\right. \\
& \left.+\left|r_{1}-\bar{r}_{1}\right|_{\mathcal{H}}+\left|r_{2}-\bar{r}_{2}\right|_{\mathcal{H}}+|r-\bar{r}|_{V_{2}}\right), \text { a.s. }
\end{aligned}
$$

Assume that $(t, \omega) \rightarrow F\left(t, p_{1}, p_{2}, p, q_{1}, q_{2}, q, r_{1}, r_{2}, r, \omega\right)$ is predictable for all $p_{1}, p_{2}, p, q_{1}, q_{2}, q, r_{1}, r_{2}, r$. 
Further we assume the following:

$$
E \int_{0}^{\infty} e^{\lambda t}|F(t, 0,0,0,0,0,0,0,0,0)|^{2} d t<\infty
$$

for all $\lambda \in \mathbb{R}$. We now consider the following backward stochastic differential equation in the unknown $\mathcal{F}_{t}$-adapted, $\mathbb{R} \times \mathbb{R} \times \mathcal{H}$-valued process $(p(t), q(t), r(t)=r(t, \cdot))$ :

$$
\begin{aligned}
d p(t) & =E\left[F\left(t, p(t), p(t+\delta), p_{t}, q(t), q(t+\delta), q_{t}, r(t), r(t+\delta), r_{t}\right) \mid \mathcal{F}_{t}\right] d t \\
& +q(t) d B(t)+\int_{\mathbb{R}_{0}} r(t, z) \tilde{N}(d t, d z)
\end{aligned}
$$

where

$$
E\left[\int_{0}^{\infty} e^{\lambda t}|p(t)|^{2} d t\right]<\infty
$$

for all $\lambda \in \mathbb{R}$.

Theorem 4.8 (Existence and uniqueness). Assume the conditions (4.69)-(4.70) are fulfilled. Then the backward stochastic differential equation (4.71) - (4.72) admits a unique solution $(p(t), q(t), r(t, \cdot))$ such that

$$
E\left[\int_{0}^{\infty} e^{\lambda t}\left\{|p(t)|^{2}+|q(t)|^{2}+\int_{\mathbb{R}_{0}}|r(t, z)|^{2} \nu(d z)\right\} d t\right]<\infty,
$$

for all $\lambda>0$.

Proof.

\section{Existence:}

\section{Step 1:}

First, assume $\mathrm{F}$ is independent of its second, third and fourth parameter.

Set $q^{0}(t):=0, r^{0}(t, \cdot):=0$. For $n \geq 1$, define $\left(p^{n}(t), q^{n}(t), r^{n}(t, \cdot)\right)$ to be the unique solution of the following BSDE:

$$
\begin{aligned}
d p^{n}(t) & =E\left[F\left(t, q^{n-1}(t), q^{n-1}(t+\delta), q_{t}^{n-1}, r^{n-1}(t, \cdot), r^{n-1}(t+\delta, \cdot), r_{t}^{n-1}(\cdot)\right) \mid \mathcal{F}_{t}\right] d t \\
& +q^{n}(t) d B(t)+\int_{\mathbb{R}_{0}} r^{n}(t, z) \tilde{N}(d t, d z) ;
\end{aligned}
$$

for $t \in[0, \infty)$ such that

$$
E\left[\int_{0}^{\infty} e^{\lambda t}\left|p^{n}(t)\right|^{2} d t\right]<\infty .
$$

The triples $\left(p^{n}(t), q^{n}(t), r^{n}(t, \cdot)\right)$ exist by Theorem 3.1 in Haadem et al. [2013].

Our goal is to show that $\left(p^{n}(t), q^{n}(t), r^{n}(t, \cdot)\right)$ forms a Cauchy sequence. By the Itô formula we 
get that

$$
\begin{aligned}
0 & =E\left[e^{\lambda t}\left|p^{n+1}(t)-p^{n}(t)\right|^{2}+\int_{t}^{\infty} \lambda e^{\lambda s}\left|p^{n+1}(s)-p^{n}(s)\right|^{2} d s\right. \\
& +\int_{t}^{\infty} e^{\lambda s}\left|q^{n+1}(s)-q^{n}(s)\right|^{2} d s \\
& +\int_{t}^{\infty} e^{\lambda s} \int_{\mathbb{R}_{0}}\left|\left(r^{n+1}(s, z)-r^{n}(s, z)\right)\right|^{2} d s \nu(d z) \\
& \left.+2 \int_{t}^{\infty} e^{\lambda s}\left(p^{n+1}(s)-p^{n}(s)\right) E\left[F^{n}(s)-F^{n-1}(s) \mid \mathcal{F}_{s}\right] d s\right] .
\end{aligned}
$$

Rearranging, using that for all $a, b \in \mathbb{R}: 2 a b \leq \frac{a^{2}}{\epsilon}+\epsilon b^{2}$ we have by the Lipschitz requirement (5.1)

$$
\begin{aligned}
& E\left[e^{\lambda t}\left|p^{n+1}(t)-p^{n}(t)\right|^{2}\right] \\
& +E\left[\int_{t}^{\infty} \lambda e^{\lambda s}\left|p^{n+1}(s)-p^{n}(s)\right|^{2} d s\right] \\
& +E\left[\int_{t}^{\infty} e^{\lambda s}\left|q^{n+1}(s)-q^{n}(s)\right|^{2} d s\right] \\
& +E\left[\int_{t}^{\infty} \int_{\mathbb{R}_{0}} e^{\lambda s}\left|\left(r^{n+1}(s, z)-r^{n}(s, z)\right)\right|^{2} \nu(d z) d s\right] \\
& \leq C_{\epsilon} E\left[\int_{t}^{\infty} e^{\lambda s}\left|p^{n+1}(s)-p^{n}(s)\right|^{2} d s\right] \\
& +\epsilon 6 E\left[\int_{t}^{\infty} e^{\lambda s}\left|q^{n}(s)-q^{n-1}(s)\right|^{2} d s\right] \\
& +\epsilon 6 E\left[\int_{t}^{\infty} e^{\lambda s}\left|q^{n}(s+\delta)-q^{n-1}(s+\delta)\right|^{2} d s\right] \\
& +\epsilon 6 E\left[\int_{t}^{\infty} e^{\lambda s} \int_{s}^{s+\delta}\left|q^{n}(u)-q^{n-1}(u)\right|^{2} d u d s\right] \\
& +\epsilon 6 E\left[\int_{t}^{\infty} e^{\lambda s}\left|r^{n}(s)-r^{n-1}(s)\right|_{\mathcal{H}}^{2} d s\right] \\
& +\epsilon 6 E\left[\int_{t}^{\infty} e^{\lambda s}\left|r^{n}(s+\delta)-r^{n-1}(s+\delta)\right|_{\mathcal{H}}^{2} d s\right] \\
& +\epsilon 6 E\left[\int_{t}^{\infty} e^{\lambda s} \int_{s}^{s+\delta}\left|r^{n}(u)-r^{n-1}(u)\right|_{\mathcal{H}}^{2} d u d s\right],
\end{aligned}
$$

where $C_{\epsilon}=\frac{C^{2}}{\epsilon}$ and we used the abbreviation

$$
F^{n}(t):=F\left(t, q^{n}(t), q^{n}(t+\delta), q_{t}^{n}, r^{n}(t, \cdot), r^{n}(t+\delta, \cdot), r_{t}^{n}(\cdot)\right)
$$


Note that

$$
\begin{aligned}
& E\left[\int_{t}^{\infty} e^{\lambda s}\left|q^{n}(s+\delta)-q^{n-1}(s+\delta)\right|^{2} d s\right. \\
& \quad \leq e^{-\lambda \delta} E\left[\int_{t}^{\infty} e^{\lambda s}\left|q^{n}(s)-q^{n-1}(s)\right|^{2} d s\right] .
\end{aligned}
$$

Using Fubini

$$
\begin{aligned}
& E\left[\int_{t}^{\infty} \int_{s}^{s+\delta} e^{\lambda s}\left|q^{n}(u)-q^{n-1}(u)\right|^{2} d u d s\right. \\
& \leq E\left[\int_{t}^{\infty} \int_{u-\delta}^{u} e^{\lambda s}\left|q^{n}(u)-q^{n-1}(u)\right|^{2} d s d u\right. \\
& \leq \frac{1}{\lambda}\left(1-e^{-\lambda \delta}\right) E\left[\int_{t}^{\infty} e^{\lambda s}\left|q^{n}(s)-q^{n-1}(s)\right|^{2} d s\right] \\
& \leq E\left[\int_{t}^{\infty} e^{\lambda s}\left|q^{n}(s)-q^{n-1}(s)\right|^{2} d s\right] .
\end{aligned}
$$

Similarly for $r^{n}-r^{n-1}$. It now follows that

$$
\begin{aligned}
& E\left[e^{\lambda t}\left|p^{n+1}(t)-p^{n}(t)\right|^{2}\right] \\
& +E\left[\int_{t}^{\infty} e^{\lambda s}\left|q^{n+1}(s)-q^{n}(s)\right|^{2} d s\right] \\
& +E\left[\int_{t}^{\infty} \int_{\mathbb{R}_{0}} e^{\lambda s}\left|\left(r^{n+1}(s, z)-r^{n}(s, z)\right)\right|^{2} \nu(d z) d s\right] \\
& \leq\left(C_{\epsilon}-\lambda\right) E\left[\int_{t}^{\infty} e^{\lambda s}\left|p^{n+1}(s)-p^{n}(s)\right|^{2} d s\right] \\
& +\epsilon 6\left(2+e^{-\lambda \delta}\right) E\left[\int_{t}^{\infty} e^{\lambda s}\left|q^{n}(s)-q^{n-1}(s)\right|^{2} d s\right] \\
& +\epsilon 6\left(2+e^{-\lambda \delta}\right) E\left[\int_{t}^{\infty} e^{\lambda s}\left|r^{n}(s)-r^{n-1}(s)\right|_{\mathcal{H}}^{2} d s\right] .
\end{aligned}
$$

Choosing $\epsilon=\frac{1}{12\left(2+e^{-\lambda \delta}\right)}$ we get

$$
\begin{aligned}
& E\left[e^{\lambda t}\left|p^{n+1}(t)-p^{n}(t)\right|^{2}\right] \\
& +E\left[\int_{t}^{\infty} e^{\lambda s}\left|q^{n+1}(s)-q^{n}(s)\right|^{2} d s\right] \\
& +E\left[\int_{t}^{\infty} \int_{\mathbb{R}_{0}} e^{\lambda s}\left|\left(r^{n+1}(s, z)-r^{n}(s, z)\right)\right|^{2} \nu(d z) d s\right] \\
& \leq\left(C_{\epsilon}-\lambda\right) E\left[\int_{t}^{\infty} e^{\lambda s}\left|p^{n+1}(s)-p^{n}(s)\right|^{2} d s\right]+\frac{1}{2} E\left[\int_{t}^{\infty} e^{\lambda s}\left|q^{n}(s)-q^{n-1}(s)\right|^{2} d s\right] \\
& +\frac{1}{2} E\left[\int_{t}^{\infty} e^{\lambda s}\left|r^{n}(s)-r^{n-1}(s)\right|_{\mathcal{H}}^{2} d s\right] .
\end{aligned}
$$


From this we deduce that

$$
\begin{aligned}
& -\frac{d}{d t}\left(e^{\left(C_{\epsilon}-\lambda\right) t} E\left[\int_{t}^{\infty} e^{\lambda s}\left|p^{n+1}(s)-p^{n}(s)\right|^{2} d s\right]\right) \\
& +e^{\left(C_{\epsilon}-\lambda\right) t} E\left[\int_{t}^{\infty} e^{\lambda s}\left|q^{n+1}(s)-q^{n}(s)\right|^{2}\right] d s \\
& +e^{\left(C_{\epsilon}-\lambda\right) t} E\left[\int_{t}^{\infty} \int_{\mathbb{R}_{0}} e^{\lambda s}\left|\left(r^{n+1}(s, z)-r^{n}(s, z)\right)\right|^{2} \nu(d z) d s\right] \\
& \leq \frac{1}{2} e^{\left(C_{\epsilon}-\lambda\right) t} E\left[\int_{t}^{\infty} e^{\lambda s}\left|q^{n}(s)-q^{n-1}(s)\right|^{2} d s\right] \\
& +\frac{1}{2} e^{\left(C_{\epsilon}-\lambda\right) t} E\left[\int_{t}^{\infty} e^{\lambda s}\left|r^{n}(s)-r^{n-1}(s)\right|_{\mathcal{H}}^{2} d s\right] .
\end{aligned}
$$

Integrating the last inequality we get that

$$
\begin{aligned}
& E\left[\int_{0}^{\infty} e^{\lambda t}\left|p^{n+1}(t)-p^{n}(t)\right|^{2} d t\right] \\
& +\int_{0}^{\infty} e^{\left(C_{\epsilon}-\lambda\right) t} E\left[\int_{t}^{\infty} e^{\lambda s}\left|q^{n+1}(s)-q^{n}(s)\right|^{2} d s\right] d t \\
& +\int_{0}^{\infty} e^{\left(C_{\epsilon}-\lambda\right) t} E\left[\int_{t}^{\infty} \int_{\mathbb{R}_{0}} e^{\lambda s}\left|\left(r^{n+1}(s, z)-r^{n}(s, z)\right)\right|^{2} \nu(d z) d s\right] d t \\
& \leq \frac{1}{2} \int_{0}^{\infty} e^{\left(C_{\epsilon}-\lambda\right) t} E\left[\int_{t}^{\infty} e^{\lambda s}\left|q^{n}(s)-q^{n-1}(s)\right|^{2} d s\right] d t \\
& +\frac{1}{2} \int_{0}^{\infty} e^{\left(C_{\epsilon}-\lambda\right) t} E\left[\int_{t}^{\infty} e^{\lambda s}\left|r^{n}(s)-r^{n-1}(s)\right|_{\mathcal{H}}^{2} d s\right] d t .
\end{aligned}
$$

So that

$$
\begin{aligned}
& \int_{0}^{\infty} e^{\left(C_{\epsilon}-\lambda\right) t} E\left[\int_{t}^{\infty} e^{\lambda s}\left|q^{n+1}(s)-q^{n}(s)\right|^{2} d s\right] d t \\
& +\int_{0}^{\infty} e^{\left(C_{\epsilon}-\lambda\right) t} E\left[\int_{t}^{\infty} \int_{\mathbb{R}_{0}} e^{\lambda s}\left|\left(r^{n+1}(s, z)-r^{n}(s, z)\right)\right|^{2} \nu(d z) d s\right] d t \\
& \leq \frac{1}{2} \int_{0}^{\infty} e^{\left(C_{\epsilon}-\lambda\right) t} E\left[\int_{t}^{\infty} e^{\lambda s}\left|q^{n}(s)-q^{n-1}(s)\right|^{2} d s\right] d t \\
& +\frac{1}{2} \int_{0}^{\infty} e^{\left(C_{\epsilon}-\lambda\right) t} E\left[\int_{t}^{\infty} e^{\lambda s}\left|r^{n}(s)-r^{n-1}(s)\right|_{\mathcal{H}}^{2} d s\right] d t
\end{aligned}
$$


This gives that

$$
\begin{aligned}
& \int_{0}^{\infty} e^{\left(C_{\epsilon}-\lambda\right) t} E\left[\int_{t}^{\infty} e^{\lambda s}\left|q^{n+1}(s)-q^{n}(s)\right|^{2} d s\right] d t \\
& +\int_{0}^{\infty} e^{\left(C_{\epsilon}-\lambda\right) t} E\left[\int_{t}^{\infty} \int_{\mathbb{R}_{0}} e^{\lambda s}\left|\left(r^{n+1}(s, z)-r^{n}(s, z)\right)\right|^{2} \nu(d z) d s\right] d t \\
& \leq \frac{1}{2^{n}} C_{3},
\end{aligned}
$$

if $\lambda>\frac{C}{\epsilon}$. It then follows from (4.75) that

$$
E\left[\int_{0}^{\infty} e^{\lambda t}\left|p^{n+1}(t)-p^{n}(t)\right|^{2} d t\right] \leq \frac{1}{2^{n}} C_{3}
$$

From (4.74) and (4.75), we now get

$$
\begin{aligned}
& E\left[\int_{t}^{\infty} \int_{\mathbb{R}_{0}} e^{\lambda s}\left|\left(r^{n+1}(s, z)-r^{n}(s, z)\right)\right|^{2} \nu(d z) d s\right] \\
& +E\left[\int_{t}^{\infty} e^{\lambda s}\left|q^{n+1}(s)-q^{n}(s)\right|^{2} d s\right] \leq \frac{1}{2^{n}} C_{3} n C_{\epsilon} .
\end{aligned}
$$

From this we conclude that there exist progressively measurable processes $(p(t), q(t), r(t, \cdot))$, such that

$$
\begin{aligned}
\lim _{n \rightarrow \infty} E\left[e^{\lambda t}\left|p^{n}(t)-p(t)\right|^{2} d t\right] & =0, \\
\lim _{n \rightarrow \infty} E\left[\int_{0}^{\infty} e^{\lambda t}\left|p^{n}(t)-p(t)\right|^{2} d t\right] & =0, \\
\lim _{n \rightarrow \infty} E\left[\int_{0}^{\infty} e^{\lambda t}\left|q^{n}(t)-q(t)\right|^{2} d t\right] & =0, \\
\lim _{n \rightarrow \infty} E\left[\int_{t}^{\infty} \int_{\mathbb{R}_{0}} e^{\lambda s}\left|r^{n}(s, z)-r(s, z)\right|^{2} \nu(d z) d s\right] & =0 .
\end{aligned}
$$

Letting $n \rightarrow \infty$ in $(4.73)$ we see that $(p(t), q(t), r(t, \cdot))$ satisfies

$$
\begin{aligned}
d p(t) & =E\left[F\left(t, q(t), q(t+\delta), q_{t}, r(t, \cdot), r(t+\delta, \cdot), r_{t}(\cdot)\right) \mid \mathcal{F}_{t}\right] d t \\
& +q(t) d B(t)+\int_{\mathbb{R}_{0}} r(t, z) \tilde{N}(d t, d z),
\end{aligned}
$$

for all $t>0$.

\section{Step 2:}

General F.

Let $p^{0}(t)=0$. For $n \geq 1$ define $\left(p^{n}(t), q^{n}(t), r^{n}(t, \cdot)\right)$ to be the unique solution to the following 
ABSDE:

$$
\begin{aligned}
d p^{n}(t) & =E\left[F\left(t, p^{n-1}(t), p^{n-1}(t+\delta), p_{t}^{n-1}, q^{n}(t), q^{n}(t+\delta), q_{t}^{n}, r^{n}(t), r^{n}(t+\delta), r_{t}^{n}\right) \mid \mathcal{F}_{t}\right] d t \\
& +q^{n}(t) d B(t)+\int_{\mathbb{R}_{0}} r^{n}(t, z) \tilde{N}(d z, d t),
\end{aligned}
$$

for $t \in[0, \infty)$. The existence of $\left(p^{n}(t), q^{n}(t), r^{n}(t, \cdot)\right)$ was proved in Step 1 .

By using the same arguments as above, we deduce that

$$
\begin{aligned}
& E\left[e^{\lambda t}\left|p^{n+1}(t)-p^{n}(t)\right|^{2}\right] \\
& +E\left[\int_{t}^{\infty} e^{\lambda s}\left|q^{n+1}(s)-q^{n}(s)\right|^{2} d s\right] \\
& +E\left[\int_{t}^{\infty} e^{\lambda s} \int_{\mathbb{R}_{0}}\left|\left(r^{n+1}(s, z)-r^{n}(s, z)\right)\right|^{2} \nu(d z) d s\right] \\
& \leq\left(C_{\epsilon}-\lambda\right) E\left[\int_{t}^{\infty} e^{\lambda s}\left|p^{n+1}(s)-p^{n}(s)\right|^{2} d s\right]+\frac{1}{2} E\left[\int_{t}^{\infty} e^{\lambda s}\left|p^{n}(s)-p^{n-1}(s)\right|^{2} d s\right] .
\end{aligned}
$$

This implies that

$$
-\frac{d}{d t}\left(e^{\left(C_{\epsilon}-\lambda\right) t} E\left[\int_{t}^{\infty} e^{\lambda s}\left|p^{n+1}(s)-p^{n}(s)\right|^{2} d s\right]\right) \leq \frac{1}{2} e^{\left(C_{\epsilon}-\lambda\right) t} E\left[\int_{t}^{\infty} e^{\lambda s}\left|p^{n}(s)-p^{n-1}(s)\right|^{2} d s\right] .
$$

Integrating from 0 to $\infty$, we get

$$
E\left[\int_{0}^{\infty} e^{\lambda s}\left|p^{n+1}(s)-p^{n}(s)\right|^{2} d s\right] \leq \frac{1}{2} \int_{0}^{\infty} e^{\left(C_{\epsilon}-\lambda\right) t} E\left[\int_{t}^{\infty} e^{\lambda s}\left|p^{n}(s)-p^{n-1}(s)\right|^{2} d s\right] d t .
$$

So if $\lambda \geq C_{\epsilon}$ then by iteration we see that

$$
E\left[\int_{0}^{\infty} e^{\lambda s}\left|p^{n+1}(s)-p^{n}(s)\right|^{2} d s\right] \leq \frac{K}{2^{n}\left(\lambda-C_{\epsilon}\right)^{n}}
$$

for some constant $K$.

\section{Uniqueness:}

In order to prove the uniqueness, we assume that there are two solution triples $\left(p^{1}(t), q^{1}(s), r^{1}(s, \cdot)\right)$ and $\left(p^{2}(t), q^{2}(s), r^{2}(s, \cdot)\right)$ to the ABSDE

$$
\begin{aligned}
d p(t) & =E\left[F\left(t, p(t), p(t+\delta), p_{t}, q(t), q(t+\delta), q_{t}, r(t), r(t+\delta), r_{t}\right) \mid \mathcal{F}_{t}\right] d t \\
& +q(t) d B(t)+\int_{\mathbb{R}_{0}} r(t, z) \tilde{N}(d t, d z) ; t \in[0, \infty),
\end{aligned}
$$


where

$$
E\left[\int_{0}^{\infty} e^{\lambda t}|p(t)|^{2} d t\right]<\infty
$$

and

$$
\lambda \geq \frac{3 C^{2}}{\epsilon}+\frac{1}{2}
$$

By the Itô formula, we have

$$
\begin{aligned}
& E\left[e^{\lambda t}\left|p^{1}(t)-p^{2}(t)\right|^{2}\right]+E\left[\int_{t}^{\infty} \lambda e^{\lambda s}\left|p^{1}(s)-p^{2}(s)\right| d s\right] \\
& +E\left[\int_{t}^{\infty} e^{\lambda s}\left|q^{1}(s)-q^{2}(s)\right|^{2} d s\right]+E\left[\int_{t}^{\infty} e^{\lambda s} \int_{\mathbb{R}_{0}}\left|r^{1}(s, z)-r^{2}(s, z)\right|^{2} d s \nu(d z)\right] \\
& =2 E\left[\int _ { t } ^ { \infty } e ^ { \lambda s } \left[\left|p^{1}(s)-p^{2}(s)\right|\right.\right. \\
& \times\left(E\left[F\left(s, p^{1}(s), p^{1}(s+\delta), p_{s}^{1}, q^{1}(s), q^{1}(s+\delta), q_{s}^{1}, r^{1}(s), r^{1}(s+\delta), r_{s}^{1}\right) \mid \mathcal{F}_{s}\right]\right. \\
& \left.\left.-E\left[F\left(s, p^{2}(s), p^{2}(s+\delta), p_{s}^{2}, q^{2}(s), q^{2}(s+\delta), q_{s}^{2}, r^{2}(s), r^{2}(s+\delta), r_{s}^{2}\right) \mid \mathcal{F}_{s}\right]\right)\right] d s \\
& \left.\left.\left.+\left|r^{1}(s)-r^{2}(s)\right|_{\mathcal{H}}^{2}+\left|r^{1}(s+\delta)-r^{2}(s+\delta)\right|_{\mathcal{H}}^{2}+\int_{s}^{s+\delta}\left|r^{1}(u)-r^{2}(u)\right|_{\mathcal{H}}^{2} d u\right)\right] d s\right] \\
& \leq 2 E\left[\int _ { t } ^ { \infty } e ^ { \lambda s } \left[\left|p^{1}(s)-p^{2}(s)\right|\right.\right. \\
& \times C\left(\left|p^{1}(s)-p^{2}(s)\right|+\left|p^{1}(s+\delta)-p^{2}(s+\delta)\right|+\int_{s}^{s+\delta}\left|p^{1}(u)-p^{2}(u)\right| d u\right. \\
& \times
\end{aligned}
$$


By the above inequalities for $(p, q, r)$ and the fact that $2 a b \leq \frac{a^{2}}{\epsilon}+\epsilon b^{2}$, we have that

$$
\begin{aligned}
& E\left[e^{\lambda t}\left|p^{1}(t)-p^{2}(t)\right|^{2}\right]+E\left[\int_{t}^{\infty} e^{\lambda s}\left|q^{1}(s)-q^{2}(s)\right|^{2} d s\right] \\
& +E\left[\int_{t}^{\infty} e^{\lambda s} \int_{\mathbb{R}_{0}}\left|r^{1}(s, z)-r^{2}(s, z)\right|^{2} d s \nu(d z)\right] \\
& \leq\left(\frac{3 C^{2}}{\epsilon}-\lambda\right) E\left[\int_{t}^{\infty} e^{\lambda s}\left|p^{1}(s)-p^{2}(s)\right|^{2} d s\right] \\
& +\left(2+e^{-\lambda \delta}\right) \epsilon E\left[\int_{t}^{\infty} e^{\lambda s}\left|p^{1}(s)-p^{2}(s)\right|^{2} d s\right] \\
& +\left(2+e^{-\lambda \delta}\right) \epsilon E\left[\int_{t}^{\infty} e^{\lambda s}\left[\left|q^{1}(s)-q^{2}(s)\right|^{2}+\left|r^{1}(s, z)-r^{2}(s, z)\right|_{\mathcal{H}}^{2}\right] d s\right] .
\end{aligned}
$$

Taking $\epsilon$ such that $\left(2+e^{-\lambda \delta}\right) \epsilon=\frac{1}{2}$

$$
\begin{aligned}
& E\left[e^{\lambda t}\left|p^{1}(t)-p^{2}(t)\right|^{2}\right]+E\left[\int_{t}^{\infty} e^{\lambda s}\left|q^{1}(s)-q^{2}(s)\right|^{2} d s\right] \\
& +E\left[\int_{t}^{\infty} e^{\lambda s} \int_{\mathbb{R}_{0}}\left|r^{1}(s, z)-r^{2}(s, z)\right|^{2} d s \nu(d z)\right] \\
& \leq\left(\frac{3 C^{2}}{\epsilon}-\lambda+\frac{1}{2}\right) E\left[\int_{t}^{\infty} e^{\lambda s}\left|p^{1}(s)-p^{2}(s)\right|^{2} d s\right] \\
& +\frac{1}{2} E\left[\int_{t}^{\infty}\left|q^{1}(s)-q^{2}(s)\right|^{2} d s\right] \\
& +\frac{1}{2} E\left[\int_{t}^{\infty}\left|r^{1}(s, z)-r^{2}(s, z)\right|_{\mathcal{H}}^{2} d s\right] .
\end{aligned}
$$


We get

$$
\begin{aligned}
& E\left[e^{\lambda t}\left|p^{1}(t)-p^{2}(t)\right|^{2}\right]+\frac{1}{2} E\left[e^{\lambda s}\left|q^{1}(s)-q^{2}(s)\right|^{2} d s\right] \\
& +\frac{1}{2} E\left[\int_{t}^{\infty} e^{\lambda s} \int_{\mathbb{R}_{0}}\left|r^{1}(s, z)-r^{2}(s, z)\right|^{2} d s \nu(d z)\right] \\
& \leq\left(\frac{3 C^{2}}{\epsilon}-\lambda+\frac{1}{2}\right) E\left[\int_{t}^{\infty} e^{\lambda s}\left|p^{1}(s)-p^{2}(s)\right|^{2} d s\right] .
\end{aligned}
$$

Using the fact that $\lambda \geq \frac{3 C^{2}}{\epsilon}+\frac{1}{2}$, we obtain for all $t \in[0, \infty)$,

$$
E\left[e^{\lambda t}\left|p^{1}(t)-p^{2}(t)\right|^{2}\right]=0
$$

which proves that $p^{1}(t)$ and $p^{2}(t)$ are indistinguishable.

Acknowledgment 4.9. We thank Salah-E.A. Mohammed for fruitful conversations. 


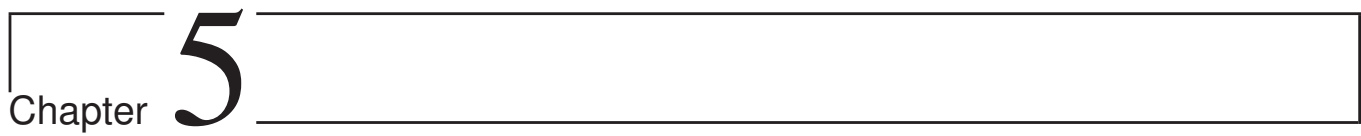

Backward Stochastic Fractional Differential

Equations and a Xaximum Principle with

Infinite Horizon Driven by a Fractional

Brownian Motion 


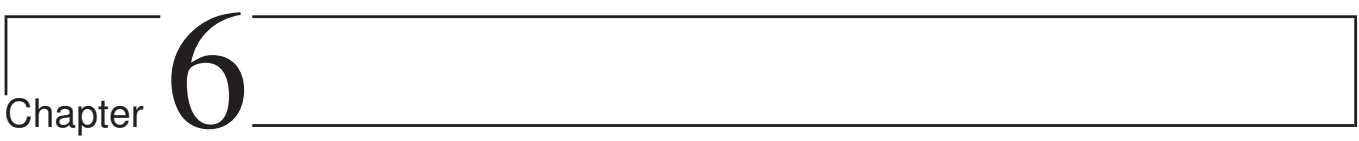

On the Construction and Malliavin

Differentiability of Lévy Noise Driven SDEs

with Singular Coefficients

With Frank Proske.

To be published in Journal of Functional Analysis. 


\section{Part II}

\section{Future Work}




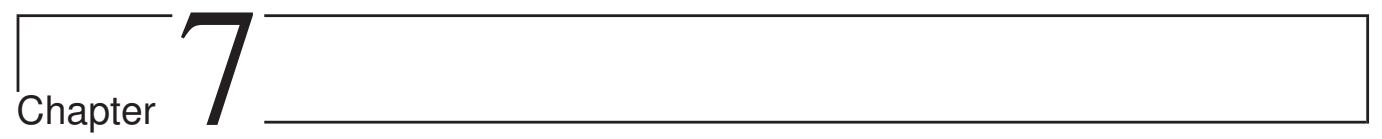

\section{Future Work}

In this chapter we briefly scetch some thoughts on further research. Many of the resu By using fractional Brownian instead of Lévy noise as in chapter 6 we get an equation which is very "noisy". Because of the interaction between the irregularities in the noise and in the solution, we may be able to smooth out ODEs and receive a much smoother solution than with other type of noise. When one works with fractional Brownian motion many of the nice equations we have with Lévy noise gets really rough. So we may need to employ different techniques and tactics. Rough paths may be the key.

\subsection{Connection with Besov Spaces}

As we have seen above, for $H>\frac{1}{2}$ it was in Zähle [1998] shown that almost surely, the sample paths of $W^{H}$ belongs to the Besov space $\mathcal{W}_{2, \infty}^{\beta}$ for $\frac{1}{2}<\beta<H$. Here $\mathcal{W}_{2, \infty}^{\beta}$ is the Besov space of all bounded measurable functions $f:[0,1] \rightarrow \mathbb{R}$ such that

$$
\int_{0}^{1} \int_{0}^{1} \frac{(f(t)-f(s))^{2}}{|-s|^{2 \beta+1}} d s d t<\infty
$$

If we try to employ the same ideas as in chapter 6 to fractional Brownian motion we soon run into troubles do to the complexity of the Itô isometry. After taking the Malliavin derivative we end up with

$$
\begin{aligned}
D_{l} X_{t} & =D_{l}\left(\int_{0}^{t} b\left(s, X_{s}\right) d s+B_{t}^{H}\right)=-\frac{\partial u}{\partial x}\left(t, X_{t}\right)+\int_{l}^{t} \frac{\partial^{2} u}{\partial x^{2}}\left(s, X_{s}\right) D_{l} X_{s} d B_{s}^{H} \\
& +M\left(\frac{\partial u}{\partial x}\left(l, X_{l}\right)\right)+M\left(\mathbf{1}_{[0, t]}\right)(l)
\end{aligned}
$$


so that

$$
\begin{aligned}
E\left(D_{l} X_{t}\right)^{2} & \leq K E\left[\frac{\partial u}{\partial x}\left(t, X_{t}\right)\right)^{2}+\int_{l}^{t}\left(M \mathbf{1}_{[l, t]} \frac{\partial^{2} u}{\partial x^{2}}\left(s, X_{s}\right) D_{l} X_{s}\right)^{2} d s \\
& +\int_{\mathbb{R}} \int_{\mathbb{R}} D_{u}\left[\left(M \mathbf{1}_{[l, t]} \frac{\partial^{2} u}{\partial x^{2}}\left(s, X_{s}\right) D_{l} X_{s}\right)\right] D_{s}\left[\left(M \mathbf{1}_{[l, t]} \frac{\partial^{2} u}{\partial x^{2}}\left(u, X_{u}\right) D_{l} X_{u}\right)\right] d(s, u) \\
& +\left(M\left(\frac{\partial u}{\partial x}\left(l, X_{l}\right)\right)\right)^{2}+\left(M \mathbf{1}_{[0, t]}(l)\right)^{2} .
\end{aligned}
$$

As mentioned above it may be helpful to turn our attention to rough paths.

\subsection{Rough Paths}

We will now work with rough paths but first we need a heat equation that suits our fractional Brownian motion.

\subsubsection{Time scaled heat equation}

This approach is a fractional version of the approach in Flandoli [2010].

Theorem 7.1. Let $\phi \in C\left([0, T] ; C_{b}^{\alpha}\left(\mathbb{R}^{d}\right)\right.$, and consider

$$
u(t, x)=\int_{0}^{t} E\left[\phi\left(s, x+W_{\left(t^{2 H}-s^{2 H}\right)}\right)\right] d s .
$$

Then $u(t, x)$ solves

$$
\frac{\partial u}{\partial t}=H t^{2 H-1} \Delta u+\phi,
$$

with $u(0, x)=0$. Further there exists $C(T)$ such that

$$
\left\|D^{2} u\right\| \leq C(T)
$$

,for some constant $K$, and

$$
\|\nabla u\|_{C_{b}^{\alpha}(T)} \leq C(T)\|\phi\|_{C_{b}^{\alpha}(T)}
$$

Proof. Note that

$$
\begin{aligned}
E\left[\phi\left(s, x+W_{\left(t^{2 H}-s^{2 H}\right)}\right)\right] & =\frac{1}{(2 \pi)^{\frac{d}{2}}\left(t^{2 H}-s^{2 H}\right)^{\frac{d}{2}}} \int_{\mathbb{R}^{d}} \phi(s, x+z) \exp \left(-\frac{|z|^{2}}{2\left(t^{2 H}-s^{2 H}\right)}\right) d z \\
& =\frac{1}{(2 \pi)^{\frac{d}{2}}\left(t^{2 H}-s^{2 H}\right)^{\frac{d}{2}}} \int_{\mathbb{R}^{d}} \phi(s, y) \exp \left(-\frac{|y-x|^{2}}{2\left(t^{2 H}-s^{2 H}\right)}\right) d y
\end{aligned}
$$


This gives us

$$
D E\left[\phi\left(s, x+W_{\left(t^{2 H}-s^{2 H}\right)}\right)\right]=\frac{1}{\left(t^{2 H}-s^{2 H}\right)} E\left[\phi\left(s, x+W_{\left(t^{2 H}-s^{2 H}\right)}\right) W_{\left(t^{2 H}-s^{2 H}\right)}\right]
$$

and

$D^{2} E\left[\phi\left(s, x+W_{\left(t^{2 H}-s^{2 H}\right)}\right)\right]=\frac{1}{\left(t^{2 H}-s^{2 H}\right)^{2}} E\left[\phi\left(s, x+W_{\left(t^{2 H}-s^{2 H}\right)}\right)\left(\left(W_{\left(t^{2 H}-s^{2 H}\right)}\right)^{2}-\left(t^{2 H}-s^{2 H}\right) I_{d}\right)\right]$.

Taking the derivative in the direction of $t$, we get

$$
\frac{\partial}{\partial t} E\left[\phi\left(s, x+W_{\left(t^{2 H}-s^{2 H}\right)}\right)\right]=\frac{H t^{2 H-1}}{\left(t^{2 H}-s^{2 H}\right)^{2}} E\left[\phi\left(s, x+W_{\left(t^{2 H}-s^{2 H}\right)}\left(\left(W_{\left(t^{2 H}-s^{2 H}\right)}\right)^{2}-\left(t^{2 H}-s^{2 H}\right) I_{d}\right)\right] .\right.
$$

So that

$$
\begin{aligned}
& \frac{\partial}{\partial t} \int_{0}^{t} E\left[\phi\left(s, x+W_{\left(t^{2 H}-s^{2 H}\right)}\right)\right] d s \\
& =\int_{0}^{t} \frac{H t^{2 H-1}}{\left(t^{2 H}-s^{2 H}\right)^{2}} E\left[\phi\left(s, x+W_{\left(t^{2 H}-s^{2 H}\right)}^{H}\right)\left(\left(W_{\left(t^{2 H}-s^{2 H}\right)}\right)^{2}-\left(t^{2 H}-s^{2 H}\right) I_{d}\right) d s\right]+\phi(t, x) \\
& =H t^{2 H-1} \Delta u+\phi(t, x)
\end{aligned}
$$

Then,

$$
\begin{aligned}
\left\|D^{2} E\left[\phi\left(s, x+W_{\left(t^{2 H}-s^{2 H}\right)}\right)\right]\right\| & \leq \frac{1}{\left(t^{2 H}-s^{2 H}\right)^{2}}\|\phi\|_{C_{b}^{\alpha}} E\left[\left|W_{\left(t^{2 H}-s^{2 H}\right)}\right|^{\alpha}\left(W_{\left(t^{2 H}-s^{2 H}\right)}^{2}+\left(t^{2 H}-s^{2 H}\right)\right]\right. \\
& \leq \frac{C\|\phi\|_{b}^{\alpha}}{\left(t^{2 H}-s^{2 H}\right)^{1-\alpha / 2}} .
\end{aligned}
$$

so that

$$
\int_{0}^{t}\left\|D^{2} E\left[\phi\left(s, x+W_{\left(t^{2 H}-s^{2 H}\right)}\right)\right]\right\| d s \leq C\|\phi\|_{C_{b}^{\alpha}} \int_{0}^{t} \frac{1}{\left(t^{2 H}-s^{2 H}\right)^{1-\alpha / 2}} d s .
$$

This theorem allows to consider the backward Kolmogorov equation.

Theorem 7.2. For all $\left.\phi \in C[0, T]: C_{b}^{\alpha}\left(\mathbb{R}^{d}\right)\right)$ there exists at least one solution to the backward Kolmogorov equation:

$$
\frac{\partial U_{b}}{\partial t}+b \nabla U_{b}+H t^{2 H-1} \Delta U_{b}=-b
$$

$u(T, x)=0$, with

$$
u \in C\left([0, T]: C_{b}^{2, \alpha^{\prime}}\left(\mathbb{R}^{d}\right)\right) \cup C^{1}\left([0, T]: C_{b}^{\alpha^{\prime}}\left(\mathbb{R}^{d}\right)\right)
$$


for all $\alpha^{\prime} \in(0, \alpha)$ and

$$
\left\|D u^{2}\right\|_{C_{b}^{\alpha^{\prime}(T)}} \leq C_{\alpha^{\prime}}\|\phi\|_{C_{b}^{\alpha}(T)}
$$

and

$$
\|\nabla u\|_{C_{b}^{\alpha}(T)} \leq C(T)\|\phi\|_{C_{b}^{\alpha}(T)}
$$

with

$$
\lim _{T \rightarrow 0} C(T)=0 .
$$

Proof. Using a standard Picard iteration tactic based on the Heat equation:

$$
\frac{\partial u^{n+1}}{\partial t}+H t^{2 H-1} \Delta u^{n+1}=-(b \cdot \nabla) u^{n}-\phi
$$

in $[0, T]$, and $u^{n+1}(T, x)=0$. From Theorem 7.1 we have a solution

$$
u^{n+1} \in C\left([0, T]: C_{b}^{2, \alpha^{\prime}}\left(\mathbb{R}^{d}\right)\right) \cup C^{1}\left([0, T]: C_{b}^{\alpha^{\prime}}\left(\mathbb{R}^{d}\right)\right),
$$

for every $\alpha^{\prime} \leq \alpha$ and $n$ with

$$
\left\|D u^{2}\right\|_{C_{b}^{\alpha^{\prime}(T)}} \leq C_{\alpha^{\prime}}\|\phi\|_{C_{b}^{\alpha}(T)}
$$

and

$$
\|\nabla u\|_{C_{b}^{\alpha}(T)} \leq C(T)\|\phi\|_{C_{b}^{\alpha}(T)}
$$

with

$$
\lim _{T \rightarrow 0} C(T)=0 .
$$

Further, we see that

$$
\begin{aligned}
\left\|(b \cdot \nabla) u^{n}+\phi\right\|_{C_{b}^{\alpha}(T)} & \leq\|\phi\|_{C_{b}^{\alpha}(T)}+2\|b\|_{C_{b}^{\alpha}(T)}\left\|\nabla u^{n}\right\|_{C_{b}^{\alpha}(T)} \\
& \leq\|\phi\|_{C_{b}^{\alpha}(T)}+2\|b\|_{C_{b}^{\alpha}(T)} C(T)\left\|(b \cdot \nabla) u^{n-1}+\phi\right\|_{C_{b}^{\alpha}(T)}
\end{aligned}
$$

Choose $T$ such that $2\|b\|_{C_{b}^{\alpha}(T)} C(T) \leq \frac{1}{2}$ and set $\nu^{n}:=(b \cdot \nabla) u^{n}+\phi$. Then we get that

$$
\begin{aligned}
\left\|\nu^{n}\right\|_{C_{b}^{\alpha}(T)} & \leq\|\phi\|_{C_{b}^{\alpha}(T)}+\frac{1}{2}\left\|\nu^{n-1}\right\|_{C_{b}^{\alpha}(T)} \\
& \leq\left(1+\frac{1}{2}+\ldots+\frac{1}{2^{n-1}}\right)\|\phi\|_{C_{b}^{\alpha}(T)}+\frac{1}{2^{n}}\left\|\nu^{n-1},\right\|_{C_{b}^{\alpha}(T)}
\end{aligned}
$$


So

$$
\left\|\nu^{n}\right\| \leq 2\|\phi\|_{C_{b}^{\alpha}(T)}
$$

This proves that

$$
\left\|D u^{2}\right\|_{C_{b}^{\alpha^{\prime}(T)}} \leq C_{\alpha^{\prime}}\|\phi\|_{C_{b}^{\alpha}(T)}
$$

and

$$
\|\nabla u\|_{C_{b}^{\alpha}(T)} \leq C(T)\|\phi\|_{C_{b}^{\alpha}(T)}
$$

with

$$
\lim _{T \rightarrow 0} C(T)=0
$$

We also have

$$
\left\|\frac{\partial u^{n+1}}{\partial t}\right\|_{C_{b}^{\alpha}(T)} \leq C
$$

We now have from Acoli-Arzelà theorem that there exists a convergent subsequence that converges uniformly to some $u$. It is easy to check that $u$ has the same regularity and bounds as $u^{n}$, with exception of $\frac{\partial u^{n}}{\partial t}$. By passing to the limit in the equation

$$
u^{n+1}(t, x)=\int_{t}^{T}\left((b \cdot \nabla) u^{n}(s, x)+H s^{2 H-1} \Delta u^{n+1}(s, x)+\phi(s, x)\right) d s,
$$

we get the same equation for $u$, which implies that

$$
\frac{\partial u}{\partial t} \in C\left([0, T]: C_{b}^{2, \alpha^{\prime}}\left(\mathbb{R}^{d}\right)\right)
$$

and that $u$ is a solution of (7.1). The proof is complete.

Corollary 7.3. We have the following representation:

$$
\begin{aligned}
\int_{0}^{t} b\left(s, X_{s}\right) d s & =u(0, x)-u\left(t, X_{t}\right)+\int_{0}^{t} \frac{\partial u}{\partial x}\left(s, X_{s}\right) \circ d B_{s}^{H} \\
& -H \int_{0}^{t} \frac{\partial^{2} u}{\partial x^{2}}\left(s, X^{H}(s)\right) s^{2 H-1} d s .
\end{aligned}
$$


Proof. Using Itô's Lemma for fBm, we get the dynamics:

$$
\begin{aligned}
u\left(t, X^{H}(t)\right) & =u(0,0)+\int_{0}^{t} \frac{\partial u}{\partial s}\left(s, X^{H}(s)\right) d s \\
& +\int_{0}^{t} \frac{\partial u}{\partial x}\left(s, X^{H}(s)\right) \circ d X^{H}(s) \\
& =\int_{0}^{t} \frac{\partial u}{\partial s}\left(s, X^{H}(s)\right) d s+\int_{0}^{t} \frac{\partial u}{\partial x}\left(s, X^{H}(s)\right) b\left(s, X^{H}(s)\right) d s \\
& +\int_{0}^{t} \frac{\partial u}{\partial x}\left(s, X^{H}(s)\right) \circ d B^{H}(s)
\end{aligned}
$$

Using Theorem 7.2 we know that the equation

$$
\frac{\partial U_{b}}{\partial t}+b \nabla U_{b}+H t^{2 H-1} \Delta U_{b}=-b
$$

has a solution. This gives us that

$$
\begin{aligned}
\int_{0}^{t} b(s, X(s)) d s & =U_{b}(0,0)-U_{b}(t, X(t))+\int_{0}^{t} \frac{\partial U_{b}}{\partial x}(t, X(t)) \circ d B^{H}(t) \\
& -H \int_{0}^{t} \frac{\partial^{2} u}{\partial x^{2}}\left(s, X^{H}(s)\right) s^{2 H-1} d s .
\end{aligned}
$$

From this we have that

$$
\begin{aligned}
X_{t} & =x+\int_{0}^{t} b\left(s, X_{s}\right) d s+B_{t}^{H}=u(0, x)-u\left(t, X_{t}\right)+\int_{0}^{t} \frac{\partial u}{\partial x}\left(s, X_{s}\right) \circ d B_{s}^{H} \\
& +B_{t}^{H}-H \int_{0}^{t} \frac{\partial^{2} u}{\partial x^{2}}\left(s, X^{H}(s)\right) s^{2 H-1} d s .
\end{aligned}
$$

Remember we have a integration of parts for Riemann-Stieltjes integrals $\int f d g$ if $f \in C^{\lambda}$ and $g \in C^{\mu}$ where $\lambda+\mu>1$. For $1-\mu<\alpha<\lambda$ we have

$$
\int_{a}^{b} f d g=(-1)^{\alpha} \int_{a}^{b} D_{a_{+}}^{\alpha} f(x) D_{b_{-}}^{1-\alpha} g_{b_{-}}(x) d x
$$

where $g_{b_{-}}(x)=g(x)-g(b)$.

As in Hu and Nualart [2009] let us define the compensated fractional derivative for $r \in[0, a]$. ( can we use fractional Laplacian instead?)

$$
\hat{D}_{a_{+}}^{\alpha} f(x)(r)=\frac{1}{\Gamma(1-\alpha)}\left(\frac{f\left(x_{r}\right)}{(r-a)^{\alpha}}+\alpha \int_{a}^{r} \frac{f\left(x_{r}\right)-f\left(x_{\theta}-\sum_{i=1}^{m} \partial_{i} f\left(x_{\theta}\right)\left(x_{r}^{i}-x_{\theta}^{i}\right)\right.}{(r-\theta)^{\alpha+1}} d \theta\right) .
$$




$$
\mathcal{D}_{b_{-}}^{1-\alpha}(x \otimes y)(r)=\frac{(-1)^{1-\alpha}}{\Gamma(\alpha)}\left(\frac{(x \otimes y)_{r, b}}{(b-r)^{1-\alpha}}+\left(1-\alpha \int_{r}^{b} \frac{(x \otimes y)_{r, s}}{(s-r)^{1-\alpha}} d s\right)\right.
$$

Lejay [2009] Friz and Victoir [2010]

Definition 7.4. Let $(x, y, x \otimes y)$ be in $M_{m, d}^{\beta}(0, T)$. Let $f: \mathbb{R}^{m} \rightarrow \mathbb{R}^{d}$ be a continuously differentiable function such that $f^{\prime}$ is locally $\lambda$-Hölder continuous where $\lambda>\frac{1}{\beta}-2$. Fix $\alpha>0$ such that $1-\beta<\alpha<2 \beta$ and $\alpha<\frac{\lambda \beta+1}{2}$. Then for any $0 \leq a<b \leq T$ we let

$$
\begin{aligned}
\int_{a}^{b} f\left(x_{r}\right) d y_{r} & =(-1)^{\alpha} \sum_{j=1}^{d} \int_{a}^{b} \hat{D}_{a_{+}}^{\alpha} f_{j}(x)(r) D_{b_{-}}^{1-\alpha} y_{b_{-}}^{j}(r) d r \\
& -(-1)^{2 \alpha-1} \sum_{i=1} \sum_{j=1} \int_{a}^{b} D_{a_{+}}^{1-\alpha} \partial_{i} f_{j}(x)(r) D_{b_{-}}^{1-\alpha} \mathcal{D}_{b_{-}}^{1-\alpha}(x \otimes y)^{i, j}(r) d r .
\end{aligned}
$$

Corollary 7.5. We have the following representation.

$$
\begin{aligned}
\int_{0}^{t} b\left(s, X_{s}\right) d s & =u(0, x)-u\left(t, X_{t}\right)+(-1)^{\alpha} \sum_{j=1}^{d} \int_{a}^{b} \hat{D}_{a_{+}}^{\alpha} u_{j}(X)(r) D_{b_{-}}^{1-\alpha} B_{b_{-}}^{H, j}(r) d r \\
& -(-1)^{2 \alpha-1} \sum_{i=1} \sum_{j=1} \int_{a}^{b} D_{a_{+}}^{1-\alpha} \partial_{i} u_{j}(X)(r) D_{b_{-}}^{1-\alpha} \mathcal{D}_{b_{-}}^{1-\alpha}\left(B^{H} \otimes B^{H}\right)^{i, j}(r) d r \\
& -H^{\prime} \int_{0}^{t} \frac{\partial^{2} u}{\partial x^{2}}\left(s, X^{H}(s)\right) s^{2 H^{\prime}-1} d s
\end{aligned}
$$


Corollary 7.6. We now have the following representation.

$$
\begin{aligned}
& \int_{0}^{t}\left(b\left(s, X_{s}\right)-b\left(s, X^{\prime}\right)\right) d s=u(0, x)-u\left(0, x^{\prime}\right)+u\left(t, X_{t}^{\prime}\right)-u\left(t, X_{t}\right) \\
& +(-1)^{\alpha} \sum_{j=1}^{d} \int_{a}^{b} \hat{D}_{a_{+}}^{\alpha}\left(u_{j}(X)(r)-u_{j}\left(X^{\prime}\right)(r)\right) D_{b_{-}}^{1-\alpha} B_{b_{-}}^{H, j}(r) d r \\
& -(-1)^{2 \alpha-1} \sum_{i=1} \sum_{j=1} \int_{a}^{b} D_{a_{+}}^{1-\alpha}\left(\partial_{i}^{2} u_{j}(X)(r)-\partial_{i}^{2} u_{j}\left(X^{\prime}\right)(r)\right) \\
& \times D_{b_{-}}^{1-\alpha} \mathcal{D}_{b_{-}}^{1-\alpha}\left(X \otimes B^{H}\right)^{i, j}(r) d r \\
& -(-1)^{2 \alpha-1} \sum_{i=1} \sum_{j=1} \int_{a}^{b} D_{a_{+}}^{1-\alpha} \partial_{i}^{2} u_{j}\left(X^{\prime}\right)(r) \\
& \times D_{b_{-}}^{1-\alpha} \mathcal{D}_{b_{-}}^{1-\alpha}\left(\left(X-X^{H}\right) \otimes B^{H}\right)^{i, j}(r) d r \\
& -H \int_{0}^{t}\left(\frac{\partial^{2} u}{\partial x^{2}}(s, X(s))-\frac{\partial^{2} u}{\partial x^{2}}\left(s, X^{\prime}(s)\right)\right) s^{2 H-1} d s \\
& =u(0, x)-u\left(0, x^{\prime}\right)+u\left(t, x_{t}^{\prime}\right)-u\left(t, X_{t}\right) \\
& +(-1)^{\alpha} \sum_{j=1}^{d} \int_{a}^{b} \frac{1}{\Gamma(1-\alpha)}\left[\frac{u\left(X_{r}\right)-u\left(x_{r}^{\prime}\right.}{(r-a)^{\alpha}}\right. \\
& +\alpha \int_{a}^{r}\left[\frac{u_{j}(X)(r)-u_{j}\left(X^{\prime}\right)(r)+u_{j}\left(X^{\prime}\right)(\theta)-u_{j}(X(\theta))}{(r-\theta)^{\alpha+1}}\right. \\
& \left.\left.+\frac{\sum \partial_{i} u\left(X_{\theta}^{\prime}\right)\left(X_{r}^{\prime}, i-X_{\theta}^{\prime, i}\right)-\partial_{i} u\left(X_{\theta}\right)\left(X_{r}^{i}-X_{\theta}^{i}\right)}{(r-\theta)^{\alpha+1}}\right] d \theta\right] D_{b_{-}}^{1-\alpha} B_{b_{-}}^{H}(r) d r \\
& -(-1)^{2 \alpha-1} \sum_{i=1} \sum_{j=1} \int_{a}^{b} D_{a_{+}}^{1-\alpha}\left(\partial_{i}^{2} u_{j}(X)(r)-\partial_{i}^{2} u_{j}\left(X^{\prime}\right)(r)\right) D_{b_{-}}^{1-\alpha} \mathcal{D}_{b_{-}}^{1-\alpha}\left(X \otimes B^{H}\right)^{i, j}(r) d r \\
& -(-1)^{2 \alpha-1} \sum_{i=1} \sum_{j=1} \int_{a}^{b} D_{a_{+}}^{1-\alpha} \partial_{i}^{2} u_{j}\left(X^{\prime}\right)(r) D_{b_{-}}^{1-\alpha} \mathcal{D}_{b_{-}}^{1-\alpha}\left(\left(X-X^{\prime}\right) \otimes B^{H}\right)^{i, j}(r) d r \\
& -H^{\prime} \int_{0}^{t}\left(\frac{\partial^{2} u}{\partial x^{2}}(s, X(s))-\frac{\partial^{2} u}{\partial x^{2}}\left(s, X^{\prime}(s)\right)\right) s^{2 H^{\prime}-1} d s
\end{aligned}
$$


For simplicity let $m=d=1$. Then we have.

$$
\begin{aligned}
& \int_{0}^{t}\left(b\left(s, X_{s}\right)-b\left(s, X^{\prime}\right)\right) d s=u(0, x)-u\left(0, x^{\prime}\right)+u\left(t, X_{t}^{\prime}\right)-u\left(t, X_{t}\right) \\
& +(-1)^{\alpha} \int_{a}^{b} \frac{1}{\Gamma(1-\alpha)}\left[\frac{u\left(X_{r}\right)-u\left(X_{r}^{\prime}\right)}{(r-a)^{\alpha}}\right. \\
& +\alpha \int_{a}^{r}\left[\frac{u(X)(r)-u\left(X^{\prime}\right)(r)+u\left(X^{\prime}\right)(\theta)-u(X(\theta))}{(r-\theta)^{\alpha+1}}\right. \\
& \left.\left.+\frac{u^{\prime}\left(X_{\theta}^{\prime}\right)\left(X_{r}^{\prime}-X_{\theta}^{\prime}\right)-u^{\prime}\left(X_{\theta}\right)\left(X_{r}-X_{\theta}\right)}{(r-\theta)^{\alpha+1}}\right] d \theta\right] D_{b_{-}}^{1-\alpha} B_{b_{-}}^{H}(r) d r \\
& -(-1)^{2 \alpha-1} \int_{a}^{b} D_{a_{+}}^{1-\alpha}\left(u^{\prime \prime}(X)(r)-u^{\prime \prime}\left(X^{\prime}\right)(r)\right) D_{b_{-}}^{1-\alpha} \mathcal{D}_{b_{-}}^{1-\alpha}\left(X \otimes B^{H}\right)(r) d r \\
& -(-1)^{2 \alpha-1} \int_{a}^{b} D_{a_{+}}^{1-\alpha} u^{\prime \prime}\left(X^{\prime}\right)(r) D_{b_{-}}^{1-\alpha} \mathcal{D}_{b_{-}}^{1-\alpha}\left(\left(X-X^{\prime}\right) \otimes B^{H}\right)(r) d r \\
& -H^{\prime} \int_{0}^{t}\left(\frac{\partial^{2} u}{\partial x^{2}}(s, X(s))-\frac{\partial^{2} u}{\partial x^{2}}\left(s, X^{\prime}(s)\right)\right) s^{2 H^{\prime}-1} d s
\end{aligned}
$$

It now follows that,

$$
\begin{aligned}
& \left|\int_{0}^{t}\left(b\left(s, X_{s}\right)-b\left(s, X^{\prime}\right)\right) d s\right| \leq \mid u(0, x)-u\left(0, x^{\prime}\right)+u\left(t, X_{t}^{\prime}\right)-u\left(t, X_{t}\right) \\
& +(-1)^{\alpha} \int_{a}^{b} \frac{1}{\Gamma(1-\alpha)}\left[\frac{u\left(X_{r}\right)-u\left(X_{r}^{\prime}\right)}{(r-a)^{\alpha}}\right. \\
& +\alpha \int_{a}^{r}\left[\frac{u(X)(r)-u\left(X^{\prime}\right)(r)+u\left(X^{\prime}\right)(\theta)-u(X(\theta))}{(r-\theta)^{\alpha+1}}\right. \\
& \left.\left.+\frac{u^{\prime}\left(X_{\theta}^{\prime}\right)\left(X_{r}^{\prime}-X_{\theta}^{\prime}\right)-u^{\prime}\left(X_{\theta}\right)\left(X_{r}-X_{\theta}\right)}{(r-\theta)^{\alpha+1}}\right] d \theta\right] D_{b_{-}}^{1-\alpha} B_{b_{-}}^{H}(r) d r \\
& -(-1)^{2 \alpha-1} \int_{a}^{b} D_{a_{+}}^{1-\alpha}\left(u^{\prime \prime}(X)(r)-u^{\prime \prime}\left(X^{\prime}\right)(r)\right) D_{b_{-}}^{1-\alpha} \mathcal{D}_{b_{-}}^{1-\alpha}\left(X \otimes B^{H}\right)(r) d r \\
& -(-1)^{2 \alpha-1} \int_{a}^{b} D_{a_{+}}^{1-\alpha} u^{\prime \prime}\left(X^{\prime}\right)(r) D_{b_{-}}^{1-\alpha} \mathcal{D}_{b_{-}}^{1-\alpha}\left(\left(X-X^{\prime}\right) \otimes B^{H}\right)(r) d r \\
& -H^{\prime} \int_{0}^{t}\left(\frac{\partial^{2} u}{\partial x^{2}}(s, X(s))-\frac{\partial^{2} u}{\partial x^{2}}\left(s, X^{\prime}(s)\right)\right) s^{2 H^{\prime}-1} d s \mid .
\end{aligned}
$$

Similar to the methods used in recent work we get that, 
Theorem 7.7.

$$
\begin{aligned}
& \left\|\int\left(u^{\prime}\left(X_{r}\right)-u^{\prime}\left(X_{r}^{\prime}\right)\right) d B_{r}^{H}\right\|_{a, b, \beta} \leq k\left|u^{\prime}\left(X_{a}\right)-u\left(X_{a}^{\prime}\right)\right|\left\|B^{H}\right\|_{a, b, \beta} \\
& +k\left(\left\|\left(X-X^{\prime}\right) \otimes B^{H}\right\|_{a, b, 2 \beta}+\left\|\left(X-X^{\prime}\right)\right\|_{a, b, \beta}\left\|B^{H}\right\|_{a, b, \beta}\right) \\
& \times\left(\left\|u^{\prime}\right\|_{\infty}+\left\|u^{\prime \prime}\right\|_{\lambda}\left\|X-X^{\prime}\right\|_{a, b, \beta}(b-a)^{\alpha \beta}\right)(b-a)^{\beta}
\end{aligned}
$$

We need the following proposition.

Lemma 7.8 (Proposition 6.4 in Hu and Nualart [2009]).

$$
\begin{aligned}
& \left\|\int u^{\prime}(X) d B^{H}-\int u^{\prime}\left(X^{\prime}\right) d B^{H}\right\|_{\beta} \leq k H_{a, b}\left(B^{H}\right)\left\|X-X^{\prime}\right\|_{a, b, \infty} \\
& +k H_{a, b}^{2}\left(B^{H}\right)\left\|X-X^{\prime}\right\|_{a, b, \beta}+k H_{a, b}^{3}\left\|\left(X-X^{\prime}\right) \otimes B^{H}\right\|_{a, b, 2 \beta}
\end{aligned}
$$

By using the above we should be able to prove.

Conjecture 7.9. (Uniqueness) Strong uniqueness holds. 


\section{Part III}

\section{Background Material}




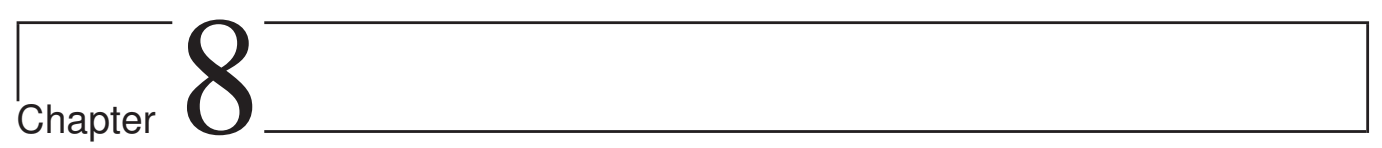

\section{Basic Stochastic Analysis}

Stochastic analysis is a deep and fascinating subject. It has many connections to other mathematical areas and has proven to be very valuable both in general mathematical theory and in practical applications. It allow us to model systems that behave randomly, which is of immense value. It gives us a consistent theory of integration for stochastic processes. Important subfields and applications of stochastic analysis include:

1. Itô integration.

2. Malliavin calculus.

3. White Noise framework.

4. Lévy processes.

5. Fractional Brownian motion.

6. Rough Paths.

7. Stochastic Processes on manifolds.

8. Risk measures.

9. Optimal control.

10. Optimal stopping.

11. Stochastic differential equations and backward stochastic differential equations.

12. Delay equations.

13. Hilbert and Banach valued stochastic processes.

14. Biology.

15. Weather analysis.

16. Quantitative finance, in which asset prices are often assumed to follow stochastic differential equations.

In the following chapters we will try to explore some of the richness of stochastic analysis. We start off in this chapter with the very basic building blocks of stochastic analysis.

The theory in this chapter is taken from Karatzas and Shreve [2000], Rogers and Williams [2000b], Rogers and Williams [2000a] and Øksendal [2007]. 
In this chapter we start with a measurable space $(\Omega, \mathbf{P}, \mathcal{F})$ and a set $T \subseteq[0, \infty)$. Let us start with some definitions.

Definition 8.1 (Filtration). A filtration is a family of $\sigma$-algebras $\left\{\mathcal{F}_{t}\right\}_{t \geq 0}$ with $\mathcal{F}_{t} \subseteq \mathcal{F}$ for each $t$ and

$$
t_{1} \leq t_{2} \Longrightarrow \mathcal{F}_{t_{1}} \subseteq \mathcal{F}_{t_{2}}
$$

In these notes we will frequently assume that our filtered probability space satisfies the usual conditions.

Definition 8.2 (Usual Conditions for a Filtered Space). A filtered probability space is said to satisfy the usual conditions if $\mathcal{F}_{0}$ contains all $\mathbf{P}$-null sets and it is right-continuous (i.e. $\mathcal{F}_{t}=\mathcal{F}_{t+}:=\bigcap_{s>t} \mathcal{F}_{s}$ for all times $\left.t\right)$.

A property that is very useful and includes processes like Brownian motion is the martingale property.

Definition 8.3 (Martingales, Super-Martingales and Sub-Martingales). A stochastic process $M: T \times \Omega \rightarrow S$ is a martingale (respectively super-martingale, sub-martingale) with respect to a filtration $\left\{\mathcal{F}_{t}\right\}_{t \geq 0}$ and probability measure $\mathbf{P}$ if

1. $\left\{\mathcal{F}_{t}\right\}_{t \geq 0}$ is a filtration of the underlying probability space $(\Omega, \mathbf{P}, \mathcal{F})$;

2. $M$ is adapted to the filtration $\left\{\mathcal{F}_{t}\right\}_{t \geq 0}$, i.e., for each $t$ in the index set $T$, the random variable $M_{t}$ is a $\mathcal{F}_{t}$-measurable function;

3. for each $t, M_{t}$ lies in the $L_{p}$ space $L_{1}\left(\Omega, \mathcal{F}_{t}, \mathbf{P} ; S\right)$, i.e.

$$
E\left(\left|M_{t}\right|\right)<+\infty
$$

4. and for all $s$ and $t$ with $s \leq t$

$$
E\left[M_{t} \mid \mathcal{F}_{s}\right]=M_{s}\left(\text { respectively } \leq M_{s} \text { and } \geq M_{s}\right)
$$

Definition 8.4 (Completion and Augmentation). Let

$$
\mathcal{N}_{t}:=\left\{F \subseteq \Omega ; \exists G \in \mathcal{F}_{t} \text { with } F \subset G, P(G)=0\right\} .
$$

For any $0 \leq t<\infty$, define

1. the completion by $\overline{\mathcal{F}}_{t}:=\sigma\left\{\mathcal{F}_{t} \cup \mathcal{N}_{t}\right\}$

2. the augmentation by $\hat{\mathcal{F}}_{t}:=\sigma\left\{\mathcal{F}_{t} \cup \mathcal{N}_{\infty}\right\}$

where $\sigma\{N\}$ for a set $N \in \mathcal{F}$ is the smallest $\sigma$-algebra generated by $N$.

\subsection{Regularity}

Martingales are one of the building blocks of stochastic analysis. As we will explore later with fractional Brownian motion, things get a lot rougher without the martingale property. One important thing to be aware of when working with martingales is to know when they have a càdlàg modification. This is why the following theorem is so essential to both the theory of martingales and stochastic analysis in general. It is one of the pillars of stochastic analysis. 
Theorem 8.5 (Doob's Regularity Theorem). Let $\left(\Omega, \mathcal{F}, \mathbf{P},\left\{\mathcal{F}_{t}\right\}\right)$ be a complete filtered space satisfying the usual. Let $\left\{N_{t}\right\}_{t \geq 0}$ be a super-martingale. Then $\left\{N_{t}\right\}_{t \geq 0}$ has an càdlàg modification, $\left\{M_{t}\right\}_{t \geq 0}$, if and only if the map $t \mapsto E\left[N_{t}\right]$ is right-continuous. Further, $\left\{M_{t}\right\}_{t \geq 0}$ is a super-martingale.

Proof. See theorem 67.7 in Rogers and Williams [2000b].

\subsection{Convergence and Local Martingales}

For all processes the aspect of convergence is of great importance. There are several important results and we list a few.

Firstly, it is important to know when $\lim _{t \rightarrow \infty} X_{t}$ exits.

Theorem 8.6 (Sub-martingale Convergence). Let $\left\{X_{t}, \mathcal{F}_{t}\right\}_{t \geq 0}$ be a right-continuous sub-martingale and assume $C:=\sup _{t \geq 0} E\left[X_{t}^{+}\right]<\infty$. Then

$$
X_{\infty}:=\lim _{t \rightarrow \infty} X_{t}
$$

exists a.e. and $E\left[\left|X_{\infty}\right|\right]<\infty$.

Proof. See Theorem 2.3.15 in Karatzas and Shreve [2000].

We have the following equivalent properties of a non-negative right-continuous sub-martingale.

Corollary 8.7. Let $\left\{X_{t}, \mathcal{F}_{t}\right\}_{t \geq 0}$ be a non-negative right-continuous sub-martingale. Then the following are equivalent:

1. $\left\{X_{t}, \mathcal{F}_{t}\right\}_{t \geq 0}$ is a uniformly integrable family of random variables;

2. $\left\{X_{t}, \mathcal{F}_{t}\right\}_{t \geq 0}$ converges in $L^{1}$ as $t \rightarrow \infty$;

3. $\left\{X_{t}, \mathcal{F}_{t}\right\}_{t \geq 0}$ converges $\mathcal{P}$-a.s. as $t \rightarrow \infty$ to an integrable random variable $X_{\infty}$ such that $\left\{X_{t}, \mathcal{F}_{t}\right\}_{0 \leq t \leq \infty}$ is a sub-martingale.

We have also have this set of equivalent conditions for convergence.

Corollary 8.8. Let $\left\{X_{t}, \mathcal{F}_{t}\right\}_{t \geq 0}$ be a non-negative right-continuous martingale. Then the following are equivalent:

1. $\left\{X_{t}, \mathcal{F}_{t}\right\}_{t \geq 0}$ is a uniformly integrable family of random variables;

2. $\left\{X_{t}, \mathcal{F}_{t}\right\}_{t \geq 0}$ converges in $L^{1}$ as $t \rightarrow \infty$;

3. $\left\{X_{t}, \mathcal{F}_{t}\right\}_{t \geq 0}$ converges $\mathcal{P}$-a.s. as $t \rightarrow \infty$ to an integrable random variable $X_{\infty}$ such that $\left\{X_{t}, \mathcal{F}_{t}\right\}_{0 \leq t \leq \infty}$ is a martingale;

4. there exists an integrable random variable $Y$, such that $X_{t}=E\left[Y \mid \mathcal{F}_{t}\right] \mathcal{P}$-a.s. for every $t \geq 0$.

A form of uniform integrability that is a required condition for several important theorems is class D and DL.

Definition 8.9 (Class D and DL). Let $\mathcal{S}$, respectively $\mathcal{S}_{a}$ be the set of all stopping times $T$ such that $P(T<\infty)=1$, respectively $P(T<a)=1$. A right-continuous process, $\left\{X_{t}, \mathcal{F}_{t}\right\}_{0 \leq t<\infty}$, is said to be of class $D$, if the family $\left\{X_{T}\right\}_{t \in \mathcal{S}}$ is uniformly integrable, or of class $D$ l, if the family $\left\{X_{T}\right\}_{t \in \mathcal{S}_{a}}$ is uniformly integrable for every $0<a<\infty$. 
The notion of martingales is often to strong so we need a weaker concept.

Definition 8.10 (Local Martingale). Let $\left\{X_{t}, \mathcal{F}_{t}\right\}_{0<t<\infty}$ be a continuous process. If there exists a non-decreasing sequence $\left\{T_{n}\right\}_{n=1}^{\infty}$ of stopping times such that $\left\{X_{t}^{(n)}=X_{t \wedge T_{n}}, \mathcal{F}_{t}\right\}_{0 \leq t<\infty}$ is a martingale for each $n \geq 1$ and $P\left[\lim _{n \rightarrow \infty} T_{n}=\infty\right]=1$, then we call $X$ a continuous local martingale. We denote all continuous local martingales, $X$, with $X_{0}=0$ by $\mathcal{M}^{c, l o c}$.

We note some cases where a local martingale is actually a martingale:

1. A local martingale of class DL is a martingale;

2. A non-negative local martingale is a super-martingale;

3. If $M \in \mathcal{M}^{c, l o c}$ and $S$ is a stopping time, then $E\left(M_{S}^{2}\right) \leq E\left[\langle M\rangle_{S}\right]$, where $M_{\infty}^{2}=\lim _{t \rightarrow \infty} M_{t}^{2}$.

\subsection{Semigroups and Generators}

An important analytical tool that connects PDEs and stochastic processes is semigroups and their generators. Let us start with a measurable space $(E, \mathcal{E})$ and assume we are given a measurable transition function, $\left\{P_{t}\right\}$. Then we define the resolvent as

$$
R_{\lambda} f(x)=\int_{0}^{\infty} e^{-\lambda t} P_{t} f(x) d t=\int_{E} f(y) R_{\lambda}(x, d y),
$$

where

$$
R_{\lambda}(x, \Gamma)=\int_{0}^{\infty} e^{-\lambda t} P_{t}(x, \Gamma) d t
$$

We need some requirements on $P_{t}$ so we define a strongly continuous contraction semigroup (SCCSG) as follows;

Definition 8.11. Let $B_{0}$ be a Banach space. A family $\left\{P_{t}\right\}$ of bounded linear operators $P_{t}$ : $B_{0} \rightarrow B_{0}$ is called a one-parameter strongly continuous contraction semigroup if

1. for all $f \in B_{0}$ we have that $\left\|P_{t} f-f\right\| \rightarrow 0$ as $t \rightarrow 0$;

2. $\left\|P_{t}\right\| \leq 1$ for $t \geq 0$;

3. $P_{t} P_{S}=P_{t+s}$ for $t, s \geq 0$.

From an SCCSG we define its generator;

Definition 8.12. Let $B_{0}$ be a Banach space and $\left\{P_{t}\right\}$ a SCCSG on $B_{0}$. the (infinitesimal) generator $\mathcal{G}$ of $\left\{P_{t}\right\}$ is the unbounded operator $\mathcal{G}: D(\mathcal{G}) \rightarrow B_{0}$ defined by: $f$ is in the domain of $\mathcal{G}, D(\mathcal{G})$ if for some $g \in B_{0}$ we have

$$
\left\|\frac{\left(P_{\epsilon} f-f\right)-g}{\epsilon}\right\| \rightarrow 0 \text { as } \epsilon \rightarrow 0,
$$

and we define $\mathcal{G} f$ to be $g$.

We are now ready to show the celebrated Hilde-Yosida theorem. 
Theorem 8.13. Let $\left\{R_{\lambda}\right\}$ be a strongly continuous contraction resolvent family on $B_{0}$. Then there exists a unique strongly continuous contraction semigroup $\left\{P_{t}\right\}$ on $B_{0}$ such that

$$
\int_{0}^{\infty} e^{-\lambda t} P_{t} f d t=R_{\lambda} f
$$

for all $f \in B_{0}$ and $\lambda>0$.

Let us now assume that $E$ is a locally compact Hausdorff space with countable base (LCCB).

Definition 8.14. A Feller-Dynkin (FD) semigroup, $\left\{P_{t}\right\}$ is a strongly continuous, sub-Markov semigroup of linear operators on $C_{0}(E)$, i.e.;

1. $P_{t}: C_{0}(E) \rightarrow C_{0}(E)$;

2. for all $f \in C_{0}(E)$ such that $0 \leq f \leq 1$ then $0 \leq P_{t} f \leq 1$;

3. $P_{s} P_{t}=P_{s+t}$ for all $s, t \geq 0$ and $P_{0}=I$;

4. for all $f \in C_{0}(E)$ we have that $\left\|P_{t} f-f\right\| \rightarrow 0$ as $t \rightarrow 0$.

We let $\mathcal{G}$ be the strong generator of the FD semigroup $P_{t}$. Then

$$
\mathcal{G} f(x)=\lim _{t \rightarrow 0} \frac{1}{t}\left[\int_{E} f(y) P_{t}(x, d y)-f(x)\right] .
$$

For a d-dimensional Markov family

$$
X_{t}^{(i)}=x_{i}+\int_{0}^{t} b_{i}\left(X_{s}\right) d s+\sum_{j=1}^{r} \int_{0}^{t} \sigma_{i, j}\left(X_{s}\right) d W_{s}^{(j)}
$$

where $W$ is an r-dimensional Brownian motion, the generator takes the form

$$
\mathcal{A} f(x)=\frac{1}{2} \sum_{i, j=1}^{d} a_{i, k}(x) \frac{\partial^{2} f}{\partial x_{i} \partial x_{k}}(x)+\sum_{i=1}^{d} b_{i}(x) \frac{\partial f}{\partial x_{i}}(x)
$$

for all test functions $f$.

\subsection{Integration with Martingales and Semi-Martingales}

To define the integral w.r.t. a martingale we are going to use the standard method of defining the integral against a class of very simple processes where the integral is easily constructed. Then by showing the Itô isometry we can extend the integral by continuity. Note that by exploiting the fact that semi-martingales are given as a martingale and a process of finite variation there is no problem of extending martingale integration to semi-martingales.

Let us start by defining this class of simple process, simply called simple processes. 
Definition 8.15. A process $X$ is called simple if there exist a strictly increasing sequence of real numbers $\left\{t_{n}\right\}_{n=0}^{\infty}$ with $t_{0}=0$ and $\lim t_{n}=\infty$, and a sequence of r.m. $\left\{\zeta_{n}\right\}$ and a constant $C$ with $\sup _{n}\left|\zeta_{n}(\omega)\right| \leq C$, such that $\zeta_{n}$ is $\mathcal{F}_{t_{n}}$-measurable for every $n$ and

$$
X_{t}(\omega)=\zeta_{0}(\omega) \mathbf{1}_{0}(t)+\sum_{i=0}^{\infty} \zeta_{i}(\omega) \mathbf{1}_{\left(t_{i}, t_{i+1}\right]}(t) .
$$

The set of simple functions is denoted by $\mathcal{L}_{0}$.

For a measurable, adapted process $X$ let

$$
[X]_{T}^{2}:=E \int_{0}^{T} X_{t}^{2} d\langle M\rangle_{t}
$$

provided the right side is finite. Let us further define $\mathcal{L}$ to be the equivalence set of all measurable, adapted processes, $X$ such that $[X]_{T}<\infty$ for all $T>0$. We define a metric on $\mathcal{L}$ by

$$
[X]:=\sum_{n=1}^{\infty} \frac{1}{2^{n}}\left(1 \wedge[X]_{n}\right) .
$$

Let $\mathcal{L}^{*}$ be the equivalence set of all progressively measurable processes, $X$, such that $[X]_{T}<\infty$ for all $T>0$ equipped with the above metric. A powerful result that will enable us to define the stochastic integral is the Doob-Meyer decomposition.

Theorem 8.16 (Doob-Meyer Decomposition). Suppose that $X=\left\{X_{t}\right\}_{t>0}$ is a right-continuous sub-martingale of class $D L$ then it admits the decomposition

$$
X_{t}=M_{t}+A_{t}
$$

where $M_{t}$ is a right-continuous martingale and $A_{t}$ is an increasing process.

Proof. See Theorem 2.4.9 in Karatzas and Shreve [2000].

We are now going to use this decomposition to construct the integral by decomposing $X^{2}$ (which is a non-negative super-martingale so of class DL) for a right-continuous martingale $X$.

Definition 8.17. Let $\mathcal{M}_{2}$ be the set of all right-continuous martingales such that $E X_{t}<\infty$ for all $t \geq 0$. For all $X \in \mathcal{M}_{2}$ we call $\langle X\rangle_{t}:=A_{t}$, where $A_{t}$ is the increasing process in the Doob-Meyer decomposition of $X^{2}$, the quadratic variation of $X$.

Let us start by looking at continuous martingales. Then we have the following result: We have that for all bounded, measurable, adapted processes there exists a sequence of simple processes, $\left\{X^{m}\right\}$ such that

$$
\sup _{T} \lim _{m \rightarrow \infty} E\left[\int_{0}^{T}\left|X_{t}^{m}-X_{t}\right|^{2} d t\right]=0 .
$$

This is just a special case of the more general proposition;

Proposition 8.18. If the function $t \mapsto\langle M\rangle_{t}(\omega)$ is absolutely continuous with respect to the Lebesgue measure for a.e. $\omega$ then $\mathcal{L}_{0}$ is dense in $\mathcal{L}$ with respect to the metric (8.2). 
Proof. See proposition 3.2.6 in Karatzas and Shreve [2000].

We also have the even more general result;

Lemma 8.19. Let $\left\{A_{t}\right\}$ be a continuous, increasing process adapted to the filtration of the martingale $M$. If $X$ is a progressively measurable process such that

$$
E\left[\int_{0}^{T} X_{t}^{2} d A_{t}\right]<\infty,
$$

for each $T>0$, then there a sequence of simple processes, $\left\{X^{m}\right\}$ such that

$$
\sup _{T>0} \lim _{m \rightarrow \infty} E\left[\int_{0}^{T}\left|X_{t}^{m}-X_{t}\right|^{2} d A_{t}\right]=0 .
$$

Proof. The proof uses a change of clock. See lemma 3.2.7 in Karatzas and Shreve [2000].

Now if we define the integral for $X \in \mathcal{L}_{0}$ on the form (8.1) by

$$
\begin{array}{r}
I_{t}(X)=\zeta_{n}\left(M_{t}-M_{t_{n}}\right)+\sum_{i=0}^{n-1} \zeta_{i}\left(M_{t_{i+1}}-M_{t_{i}}\right) \\
=\sum_{i=0}^{\infty} \zeta_{i}\left(M_{t \wedge t_{i+1}}-M_{t \wedge t_{i}}\right),
\end{array}
$$

for all $t \geq 0$ where $n$ is such that $t_{n} \leq n \leq t_{n+1}$. Now, the following properties are simple to deduce;

1. $I_{0}(X)=0$;

2. $E\left[I_{t}(X) \mid \mathcal{F}_{s}\right]=I_{s}(X)$;

3. $E\left[I_{t}(X)^{2}\right]=E\left[\int_{o}^{t} X_{u}^{2} d\langle M\rangle_{u}\right]$;

4. ||$I(X) \mid=[X]$;

5. $E\left[\left(I_{t}(X)-I_{s}(X)\right)^{2} \mid \mathcal{F}_{s}\right]=E\left[\int_{s}^{t} X_{u}^{2} d\langle M\rangle_{u} \mid \mathcal{F}_{s}\right]$

6. $I(\alpha X+\beta Y)=\alpha I(X)+\beta I(Y)$, for $\alpha, \beta \in \mathbb{R}$

It is now easily, by continuity from the Itô isometry, to extend the linear operator $I$ to $\mathcal{L}^{*}$.

Definition 8.20. For $X \in \mathcal{L}^{*}$ the stochastic integral with respect to the martingale $M \in \mathcal{M}_{2}^{c}$ is the unique, square-integrable martingale $I(X)$ such that $\lim _{n \rightarrow \infty}\left\|I\left(X^{n}\right)-I(X)\right\|=0$. We let

$$
I_{t}(X)=\int_{0}^{t} X_{s} d M_{s}
$$




\subsection{Some Useful Results}

A useful theorem is the Burkholder-Davis-Gundy Inequalities. To keep the notation a little simpler let us define

$$
M_{t}^{*}:=\max _{0 \leq s \leq t}\left|M_{s}\right|
$$

Then we have the following bounds for a continuous local-martingale.

Theorem 8.21. Let $M \in \mathcal{M}^{c, \text { loc }}$. For every $m>0$ there exist positive constants $k_{m}, K_{m}$ such that

$$
k_{m} E\left[\langle M\rangle_{T}^{m}\right] \leq E\left[\left(M_{T}^{*}\right)^{2 m}\right] \leq K_{m} E\left[\langle M\rangle_{T}^{m}\right]
$$

for every stopping time $T$.

Proof. See theorem 3.3.28 in Karatzas and Shreve [2000].

Note that if we let $m=\frac{1}{2}$ and $E\left[\sqrt{\langle M\rangle_{a}}\right]<\infty$ for all $0<a<\infty$ then $E\left[\sup _{0 \leq t \leq}\left|M_{t}\right|\right]<$ $\infty$. So $M$ is of class DL and hence a martingale.

Next theorem shows us that all continuous local martingales are in fact stochastic integrals with respect to Brownian motion.

Theorem 8.22. Suppose $M=\left\{M_{t}^{1}, \ldots, M_{t}^{d}\right\}$ is defined on a probability space $(\Omega, \mathcal{F}, \mathbf{P})$ with $M^{i} \in \mathcal{M}^{c, l o c}$. Suppose all cross-variations $\left\langle M^{i}, M^{j}\right\rangle_{t}$ is absolutely continuous with respect to the the Lebesgue measure. Then there is an extension $(\tilde{\Omega}, \tilde{\mathcal{F}}, \tilde{\mathbf{P}})$ on which there exists a ddimensional Brownian motion $W$ and a matrix $X=\left\{X_{t}^{(i, k)}\right\}$ of measurable adapted processes with

$$
\tilde{\mathbf{P}}\left[\int_{0}^{t}\left(X_{s}^{(i, k)}\right)^{2} d s<\infty\right]=1
$$

such that we have

$$
M_{t}^{i}=\sum_{k=1}^{d} \int_{0}^{t} X_{s}^{(i, k)} d W_{s}^{k}
$$

and

$$
\left\langle M_{t}^{i}, M_{t}^{j}\right\rangle=\sum_{k=1}^{d} \int_{0}^{t} X_{s}^{(i, k)} X_{t}^{(j, k)} d s .
$$

In the above theorem we started without a Brownian motion. The next theorem tells us that given a Brownian motion then every martingale (martingale with respect to the filtration generated by the Brownian motion) can be represented as a integral of a progressively measurable function against the given Brownian motion.

Theorem 8.23. Let $W=\left\{W_{t}^{1}, \ldots, W_{t}^{d}, \mathcal{F}_{t}\right\}$ be a d-dimensional Brownian motion on a probability space $(\Omega, \mathcal{F}, \mathbf{P})$ and let $\left\{\mathcal{F}_{t}\right\}$ be the augmentation of the filtration, $\left\{\mathcal{F}_{t}^{W}\right\}$ generated by the 
Brownian motion $W$. Then for any square integrable martingale $M=\left\{M_{t}^{1}, \ldots, M_{t}^{d}, \mathcal{F}_{t}\right\}$ with $M_{0}=0$ and $R C L L$ paths there exists progressively measurable processes $Y=\left\{Y_{t}^{1}, \ldots, Y_{t}^{d}, \mathcal{F}_{t}\right\}$ such that

$$
E\left[\int_{0}^{T}\left(Y_{t}^{(j)}\right)^{2} d t\right]<\infty
$$

for all $0<T<\infty$ and

$$
M_{t}=\sum_{k=1}^{d} \int_{0}^{t} Y_{s}^{(j)} d W_{s}^{j}
$$

Proof. See theorem 3.4.15 in Karatzas and Shreve [2000].

By using time change Knight has shown that we can represent every continuous local martingale as a Brownian motion.

Theorem 8.24. Let $M=\left\{M_{t}^{1}, \ldots, M_{t}^{d}, \mathcal{F}_{t}, 0 \leq t<\infty\right\}$ be a continuous, adapted process with $M^{(i)} \in \mathcal{M}^{c, l o c}, \lim _{t \rightarrow \infty}\left\langle M^{(i)}\right\rangle_{t}=\infty$ and

$$
\left\langle M^{(i)}, M^{(j)}\right\rangle_{t}=0
$$

for $1 \leq i \neq j \leq d, 0 \leq t<\infty$. Define

$$
T_{i}(s)=\inf \left\{t \geq 0 ;\left\langle M^{(i)}\right\rangle_{t}>s\right\}
$$

for $0 \leq s<\infty, 1 \leq i \leq d$, so that for each $i$ and $s$ the random time $T_{i}(s)$ is a stopping time for the right-continuous filtration $\left\{\mathcal{F}_{t}\right\}$. then the processes

$$
B_{s}^{(i)}:=M_{T_{i}(s)}^{(i)}
$$

for $0 \leq s<\infty, 1 \leq i \leq d$, are independent standard, one-dimensional Brownian motions.

\subsection{Connections with Deterministic PDE}

Let $D \subset \mathbb{R}^{d}$ be open. A mapping, $u$, of $D$ into $\mathbb{R}$ is called harmonic in $D$ if $u \in C^{2}$ and

$$
\Delta u:=\sum_{i=1}^{d} \frac{\partial^{2} u}{\partial x_{i}^{2}}=0 \text { in } D .
$$

Every harmonic function is is of class $C^{\infty}$ and has the mean-value property.

Definition 8.25. We say that the function $u: D \rightarrow \mathbb{R}$ has the mean-value property if, for every $a \in D$ and $0<r<\infty$ such that $a+\bar{B}_{r} \subset D$, we have

$$
u(a)=\int_{\partial B_{r}} u(a+x) \mu_{r}(d x),
$$


where

$$
\mu_{r}(d x)=P^{0}\left[W_{\tau_{B_{r}}} \in d x\right]
$$

and

$$
\tau_{D}:=\left\{t \geq 0 ; W_{t} \in D^{c}\right\}
$$

If $u$ is harmonic in $D$ it has the mean-value property. The converse is also true.

Proposition 8.26. If u maps $D$ into $\mathbb{R}$ han has the mean-value property, then is of class $C^{\infty}$ and is harmonic.

Proof. See proposition 4.2.5 in Karatzas and Shreve [2000].

It is easy to see that the transition density of a one-dimensional Brownian family $\left\{W_{t}\right\}$

$$
p(t ; x, y):=\frac{1}{d y} P\left[W_{t} \in d y\right]=\frac{1}{\sqrt{2 \pi t}} e^{-\frac{(x-y)^{2}}{2 t}},
$$

satisfies the heat equation

$$
\frac{\partial p}{\partial t}=\frac{1}{2} \frac{\partial^{2} p}{\partial x^{2}}
$$




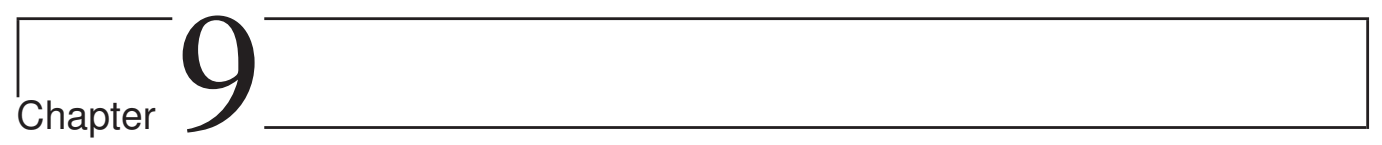

\section{Lévy Processes}

Brownian motion is often a too simple model of real world processes. In many situations we need a model that replicates sudden jumps of the system. As we see in the chapter on risk measures, we often need to account for longer tails in the distributions. To this end we extend the theory to a larger class called Lévy-processes. Lévy-processes are extensively employed in modern models as for example in financial mathematics. For this reason Lévy processes are an extensively studied class of stochastic processes. One reason for the usefulness of Lévy processes is that it is an extension of Brownian motion that gives us discontinuous paths while still belonging to important superclasses. Lévy processes are for example relatively easily used as integrators by extending the theory for continuous martingales. Many frameworks developed for Brownian motion like white noise calculus, Malliavin calculus and optimization has been given their respective extensions to Lévy processes.

This section is based on Applebaum [2009], Bertoin [2005] and Nunno et al. [2009].

\subsection{Basics}

Given a complete probability space, $(\Omega, \mathcal{F}, P)$, a Lévy process is defined as follows.

Definition 9.1. A stochastic process $L(t) \in \mathbb{R}^{d}, t \geq 0$ is called a Lévy process if the following properties hold:

1. $L(0)=0$ P-a.s.,

2. the process has independent increments, that is, for all $t>0$ and $h>0$, the increment $L(t+h)-L(h)$ is independent of $L(s)$ for all $s \leq t$,

3. the process has stationary increments, that is, for all $h>0$, the increment $L(t+h)-L(h)$ has the same law as $L(h)$,

4. the process is stochastically continuous, that is, for every $t>0$ an $\epsilon>0$ we have that $\lim _{s \rightarrow t} P\{|L(t)-L(s)|>\epsilon\}=0$,

5. the paths of the process are càdlàg, that is, the trajectories are right-continuous with existing left limits.

Now, define the jump of $L$ at time $t$ as

$$
\Delta L(t):=L(t)-L\left(t^{-}\right) .
$$


Let $\mathbb{R}_{0}^{d}:=\mathbb{R}^{d} \backslash\{0\}$ and let $\mathcal{B}\left(\mathbb{R}_{0}^{d}\right)$ be the Borel- $\sigma$-algebra on $\mathbb{R}_{0}^{d}$. Further, we now introduce a Poisson random measure on $\mathcal{B}([0, \infty)) \times \mathcal{B}\left(\mathbb{R}_{0}^{d}\right)$ by

$$
N(t, U):=\sum_{0 \leq s \leq t} \mathbf{1}_{U}(\Delta L(s))
$$

for $U \in \mathcal{B}\left(\mathbb{R}_{0}^{d}\right)$. This is the jump measure of $\eta$. The Lévy measure $\nu$ of $\eta$ is defined by

$$
\nu(U):=E[N(1, U)]
$$

for $U \in \mathcal{B}\left(\mathbb{R}_{0}^{d}\right)$.

It can be shown that the characteristic function of a Lévy process is given by the following Lévy-Khintchine formula (see e.g. Bertoin [2005]):

$$
E[\exp (i\langle L(t), u\rangle)]=\exp (-t \Psi(u)), u \in \mathbb{R}^{d}, t \geq 0,
$$

where $\Psi$ is the characteristic exponent

$$
\Psi(u)=-\int_{\mathbb{R}^{d}}\left(e^{i\langle u, y\rangle}-1-i\langle u, y\rangle \mathbf{1}_{\{|y| \leq 1\}}\right) \nu(d y) .
$$

Let us define the compensated jump measure $\widetilde{N}$ by

$$
\tilde{N}(d s, d z):=N(d s, d z)-\nu(d z) d s .
$$

It turns out that Lévy processes have the following representation:

Theorem 9.2 (The Lévy-Itô decomposition). Let $L$ be a Lévy process. Then $L$ admits the following integral representation

$$
\eta(t)=a t+\sigma W(t)+\int_{0}^{t} \int_{|z|<1} z \tilde{N}(d s, d z)+\int_{0}^{t} \int_{|z|>1} z N(d s, d z)
$$

for some $a \in \mathbb{R}^{d}, \sigma \in \mathbb{R}^{d \times d}$ and a standard Wiener process $W(t), t \geq 0$.

\subsection{Semigroups and Generators}

Let us recall the infinitesimal generator $\mathcal{L}$ of the Lévy processes $L_{t}, t \geq 0$ :

The infinitesimal generator of $L_{t}, t \geq 0$ is the operator $\mathcal{L}$, which is defined to act on suitable functions $f$ of some Banach space such that

$$
\mathcal{L} f(x)=\lim _{t \rightarrow 0^{+}} \frac{E^{x}\left[f\left(L_{t}\right)\right]-f(x)}{t}
$$

exists. 


\subsection{Stochastic Integration}

\subsubsection{Random Measures}

Definition 9.3. A random measure is a measure-valued random element.Let $X$ be a complete separable metric space and $\mathfrak{B}(X)$ the $\sigma$-algebra of its Borel sets. A Borel measure $\mu$ on $X$ is boundedly finite if $\mu(A)<\infty$ for every bounded Borel set $A$. Let $M_{X}$ be the space of all boundedly finite measures on $\mathfrak{B}(X)$. Let $(\Omega, \mathcal{F}, \mathcal{P})$ be a probability space, then a random measure maps from this probability space to the measurable space $\left(M_{X}, \mathfrak{B}\left(M_{X}\right)\right)$.

Let $X$ be a Lévy process and $N$ the associated Poisson random measure. Let $f$ be a Borel measurable function from $\mathbb{R}^{d}$ to $\mathbb{R}^{d}$. Then for a set, $A$, bounded below and $t>0$ we define the Poisson integral:

$$
\int_{A} f(x) N(t, d x)=\sum_{x \in A} f(x) N(t,\{x\}) .
$$

Note that the right hand side is a finite sum since $X$ is right-continuous and $A$ is bounded below. Let $M$ be a martingale-valued measure. Assume further that;

1. $M(\{0\}, A)=0$;

2. $M((s, t], A)$ is independent of $\mathcal{F}_{s}$;

3. There exists a $\sigma$-finite measure $\rho$ on $\mathbb{R}^{+} \times E$ such that

$$
E\left(M(t, A)^{2}\right)=\rho(t, A)
$$

for all $0 \leq s<t<\infty$, and $A \in(\mathbb{R})$.

A martingale valued measures satisfying the above conditions is said to be of $(2, \rho)$-type. Let $S=\mathbb{R}^{+} \times E$ where $E \in \mathcal{B}\left(\mathbb{R}^{d}\right)$. For a $(2, \rho)$-type martingale-valued measure and $T>0$ define $H_{2}(T, E)$ to be the linear space of all equivalence classes of functions $F:[0 ; T] \times E \times \Omega \rightarrow \mathbb{R}$ which satisfies:

1. $F$ is predictable;

2. $\int_{0}^{T} \int_{E} E\left[|F(t, x)|^{2}\right] \rho(d t, d x)<\infty$.

Define an inner product $\langle\cdot, \cdot\rangle_{T, \rho}$ on $H_{2}(T, E)$ by

$$
\left.\langle F, G\rangle_{T, \rho}:=\int_{0}^{T} \int_{E} E[(F(t, x), G(t, x))] \rho(d t, d x)\right) .
$$

Lemma 9.4. $H_{2}(T, E)$ is a real Hilbert space.

Proof. See Lemma 4.1.3 in Applebaum [2009].

Define $S(T, E)$ to be all simple processes in $H_{2}(T, E)$. then we have the following:

Lemma 9.5. $S(T, E)$ is dense in $H_{2}(T, E)$.

Proof. See Lemma 4.1.4 in Applebaum [2009]. 
For $F \in S(T, E)$ and some $m, n \in \mathbb{R}$ we write

$$
F=\sum_{j, k=1}^{m, n} F_{k}\left(t_{j}\right) \mathbf{1}_{A_{k}}
$$

and define

$$
I_{T}(F):=\sum_{j, k=1}^{m, n} F_{k}\left(t_{j}\right) M\left(\left(t_{j}, t_{j+1}\right], A_{k}\right) .
$$

It is now easy to see that

$$
E\left[I_{T}(F)^{2}\right]=\int_{0}^{T} \int_{E} E\left[|F(t, x)|^{2}\right] \rho(d t, d x)
$$

and

$$
E\left[I_{T}(F)\right]=0 .
$$

Since $I_{T}$ is a linear isometry and $S(T, E)$ is dense in $H_{2}(T, E)$ we can extend it to the whole of $H_{2}(T, E)$. This extension is called the Itô stochastic integral of $F \in H_{2}(T, E)$. 


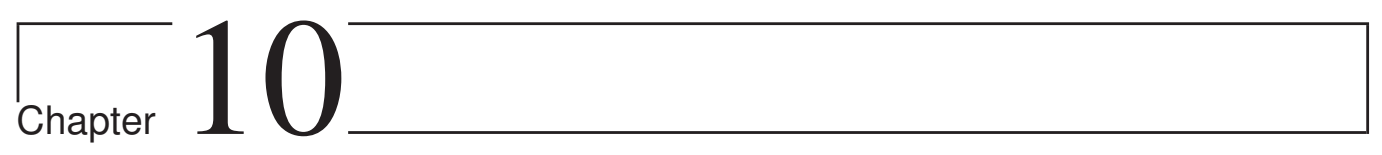

\section{Optimal Control}

Many real world problems is about finding the optimal way to control a dynamic system. There are two principal approaches to optimal control problems, the Pontryagin's maximum principle and Bellman's dynamic programming. The Pontryagin principle was formulated in 1956 by the Russian mathematician Lev Semenovich Pontryagin. The Euler-Lagrange equation of the calculus of variations is a special case of this principle. The principle states informally that a function called the Hamiltonian must be minimized over $\mathcal{U}$, the set of all permissible controls. Separately and independently Richard Bellman discovered a necessary condition for optimality. This method has become known as Bellman's dynamic programming. The point with the dynamic programming method is to breaks the decision problem into smaller subproblems. Start by considering the first decision separately, setting aside all future decisions. Then solve next subproblem until a solution of the original problem is found.

This chapter is based on Yong and Zhou [1999] and Øksendal and Sulem [2000].

\subsection{The Maximum Principle}

We start with the maximum principle. One big advantage of the maximum principle is that it can be used for non-Markovian systems. This principle will give us a set of adjoint equations and a function called the Hamiltonian. First we give a general version. Later we give a simpler version by requiring concavity.

\subsubsection{A General Maximum Principle for Brownian Motions}

Let $\left(\Omega, \mathcal{F},\{\mathcal{F}\}_{t \geq 0}, P\right)$ be a given filtered probability space satisfying the usual conditions on which a m-dimensional Brownian motion, $B(t)$, is given. Consider the following stochastic controlled system:

$$
\left\{\begin{array}{l}
d x(t)=b(t, x(t), u(t)) d t+\sigma(t, x(t), u(t)) d B(t), \quad t \in[0, T] \\
x(0)=x_{0}
\end{array}\right.
$$

with a cost functional

$$
J(u(\cdot))=E\left[\int_{0}^{T} f(t, x(t), u(t)) d t+h(x(T))\right] .
$$


Let $\phi(t, x, u):=(b(t, x, u), f(t, x, u), h(x))$. Assume that

1. $(U, d)$ is a separable metric space and $0<T<\infty$.

2. The functions $\sigma:[0, T] \times \mathbb{R}^{n} \times U \rightarrow \mathbb{R}^{n \times m}, h: \mathbb{R}^{n} \mapsto \mathbb{R}, b:[0, T] \times \mathbb{R}^{n} \times U \rightarrow \mathbb{R}^{n}$, $f:[0, T] \times \mathbb{R}^{n} \times U \rightarrow \mathbb{R}$ are measurable, and there exist a constant $L>0$ and a modulus of continuity $\bar{\omega}:[0, \infty) \rightarrow[0, \infty)$ such that

$$
\begin{cases}|\phi(t, x, u)-\phi(t, \hat{x}, \hat{u})| \leq L|x-\hat{x}|+\bar{\omega}(d(u, \hat{u})), & \text { for all } t \in[0, T], x, \hat{x} \in \mathbb{R}^{n}, u, \hat{u} \in U \\ |\phi(t, 0, u)| \leq L, & \text { for all }(t, u) \in[0, T] \times U .\end{cases}
$$

3. The functions $b, \sigma, f$ and $h$ are $C^{2}$ in $x$, and there exists a constant $L>0$ and a modulus of continuity $\bar{\omega}:[0, \infty) \rightarrow[0, \infty)$ such that

$$
\left|\phi_{x}(t, x, u)-\phi_{x}(t, \hat{x}, \hat{u})\right| \leq L|x-\hat{x}|+\bar{\omega}(d(u, \hat{u}))
$$

and

$$
\left|\phi_{x x}(t, x, u)-\phi_{x x}(t, \hat{x}, \hat{u})\right| \leq \bar{\omega}(|x-\hat{x}|+d(u, \hat{u}))
$$

for all $t \in[0, T], x, \hat{x} \in \mathbb{R}^{n}, u, \hat{u} \in U$.

4. $\left\{\mathcal{F}_{t}\right\}_{t \geq 0}$ is the natural filtration generated by $B(t)$, augmented by all the $\mathbf{P}$-null sets in $\mathcal{F}$. We let

$$
\mathcal{U}[0, T]:=\left\{u:[0, T] \times \Omega \rightarrow U \mid u \text { is }\left\{\mathcal{F}_{t}\right\} \text {-adapted }\right\}
$$

We now have the Stochastic Maximum Problem:

Problem 10.1. Minimize (10.2) over $\mathcal{U}[0, T]$.

Any $\hat{u}(\cdot) \in \mathcal{V}[0, T]$ satisfying

$$
J(\hat{u}(\cdot))=\inf _{u(\cdot) \in \mathcal{U}[0, T]} J(u(\cdot))
$$

is called an optimal control.

\subsubsection{Adjoint Equations}

Let us define the following backward stochastic differential equation, the adjoint equation, as:

$$
\left\{\begin{aligned}
d p(t)= & -\left\{b_{x}(t, \hat{x}(t), \hat{u}(t))^{T} p(t)+\sum_{j=1}^{m} \sigma_{x}^{j}(t, \hat{x}(t), \hat{u}(t))^{T} q_{j}(t)-f_{x}(t, \hat{x}(t), \hat{u}(t))\right\} d t \\
& +q(t) d W(t), t \in[0, T], \\
p(T)= & -h_{x}(\hat{x}(T)),
\end{aligned}\right.
$$

where the Hamiltonian, $H$, is defined as:

$$
H(t, x, u, p, P):=\frac{1}{2} \operatorname{tr}\left(P \sigma(t, x, u) \sigma(t, x, u)^{T}\right)+\langle p, b(t, x, u)\rangle-f(t, x, u)
$$


for all $(t, x, u, p, P) \in[0, T] \times \mathbb{R}^{n} \times U \times \mathbb{R}^{n} \times \mathcal{S}^{n}$. In addition let

$$
\left\{\begin{aligned}
d P(t)= & -\left\{b_{x}(t, \hat{x}(t), \hat{u}(t))^{T} P(t)+P(t) b_{x}(t, \hat{x}(t), \hat{u}(t))\right. \\
& +\sum_{j=1}^{m} \sigma_{x}^{j}(t, \hat{x}(t), \hat{u}(t))^{T} P(t) \sigma_{x}^{j}(t, \hat{x}(t), \hat{u}(t)) \\
& +\sum_{j=1}^{m}\left\{\sigma_{x}^{j}(t, \hat{x}(t), \hat{u}(t))^{T} Q_{j}(t)+Q_{j}(t) \sigma_{x}^{j}(t, \hat{x}(t), \hat{u}(t))\right. \\
& \left.+H_{x x}(t, \hat{x}(t), \hat{u}(t), p(t), q(t))\right\} d t \\
& +\sum_{j=1}^{m} Q_{j}(t) d W^{j}(t), t \in[0, T], \\
& -h_{x x}(\hat{x}(T)) .
\end{aligned}\right.
$$

To take into account the risk related to the diffusion coefficient, we need to introduce the generalized Hamiltonian:

$$
G(t, x, u, p, P):=\frac{1}{2} \operatorname{tr}\left(P \sigma(t, x, u) \sigma(t, x, u)^{T}\right)+\langle p, b(t, x, u)\rangle-f(t, x, u)
$$

for all $(t, x, u, p, P) \in[0, T] \times \mathbb{R}^{n} \times U \times \mathbb{R}^{n} \times \mathcal{S}^{n}$. Define the $\mathcal{H}$-function as follows:

$$
\mathcal{H}(t, x, u):=G(t, x, u, p(t), P(t))+\operatorname{tr}\left\{\sigma(t, x, u)^{T}[q(t)-P(t) \sigma(t, \hat{x}(t), \hat{u}(t))]\right\}
$$

We are now ready to present the main theorem, namely the Stochastic Maximum Principle:

Theorem 10.2 (Stochastic Maximum Principle). Under the above assumptions let $(\hat{x}(\cdot), \hat{u}(\cdot))$ be an optimal pair of problem (10.1). Then there are pair of processes

$$
\left\{\begin{array}{l}
(p(\cdot), q(\cdot)) \in L^{2}\left([0, T], \mathbb{R}^{n}\right) \times\left(L^{2}\left([0, T], \mathbb{R}^{n}\right)\right)^{m} \\
(P(\cdot), Q(\cdot)) \in L^{2}\left([0, T], \mathcal{S}^{n}\right) \times\left(L^{2}\left([0, T], \mathcal{R}^{n}\right)\right)^{m}
\end{array}\right.
$$

satisfying the first-order and second-order adjoint equations (10.3) and (10.5), respectively, such that

$$
\begin{aligned}
& H(t, \hat{x}(t), \hat{u}(t), p(t), q(t))-H(t, \hat{x}(t), u(t), p(t), q(t)) \\
& -\frac{1}{2} \operatorname{tr}\left(\{\sigma(t, \hat{x}(t), \hat{u}(t))-\sigma(t, \hat{x}(t), u(t))\}^{T} P(t)\right. \\
& \times\{\sigma(t, \hat{x}(t), \hat{u}(t))-\sigma(t, \hat{x}(t), u(t))\} \geq 0
\end{aligned}
$$

for all $u \in U$ and a.e $t \in[0, T]$ or, equivalently,

$$
\mathcal{H}(t, \hat{x}(t), \hat{u}(t))=\max _{u \in U} \mathcal{H}(t, \hat{x}(t), u(t)) .
$$

for a.e. $t \in[0, T], \mathbf{P}$-a.s.

Proof. See Theorem 3.2 in Yong and Zhou [1999].

In some cases the requirements simplifies quite a bit. 
1 The diffusion does not contain the control variable. In this case (10.8) reduces to

$$
H(t, \hat{x}(t), \hat{u}(t), p(t), q(t))=\max _{u \in U} H(t, \hat{x}(t), u(t), p(t), q(t))
$$

for a.e. $t \in[0, T], \mathbf{P}$-a.s.

1. The control domain $U \subseteq \mathbb{R}^{k}$ is convex and all the coefficients are $C^{1}$ in $u$. Then (10.8) reduces to

$$
\left\langle H_{u}(t, \hat{x}(t), \hat{u}(t), p(t), q(t)), u-\hat{u}(t)\right\rangle \leq 0,
$$

for all $u \in U$, a.e. $t \in[0, T]$, P-a.s.

\subsubsection{A Concave Maximum Principle for Jump Diffusions}

Under some further restraints we get a much simpler version of the maximum principle. Consider a jump diffusion on the form

$$
\left\{\begin{aligned}
d x(t) & =b(t, x(t), u(t)) d t+\sigma(t, x(t), u(t)) d B(t), t \in[0, T] \\
& +\int_{\mathbb{R}^{l}} \gamma\left(t, X\left(t^{-}\right), u\left(t^{-}\right), z\right) \tilde{N}(d t, d z)
\end{aligned}\right.
$$

with a cost functional

$$
J(u(\cdot))=E\left[\int_{0}^{T} f(t, x(t), u(t)) d t+g(x(T))\right] .
$$

The problem now is:

Problem 10.3. Find $u^{*} \in \mathcal{A}$ such that

$$
J\left(u^{*}\right)=\sup _{u \in \mathcal{A}} J(u)
$$

Now, define the Hamiltonian as

$$
\begin{aligned}
H(t, x, u, p, q, r) & :=f(t, x, u)+b(t, x, u)^{T}+\operatorname{tr}\left(\sigma(t, x, u)^{T} q\right) \\
& +\sum_{j=1}^{l} \sum_{i=1}^{n} \int_{\mathbb{R}} \gamma_{i j}\left(t, x, u, z_{j}\right) r_{i j} \nu_{j}\left(d z_{j}\right),
\end{aligned}
$$

Let the adjoint equation be the triple $p(t) \in \mathbb{R}^{n}, q(t) \in \mathbb{R}^{n \times l}$ and $r(t, z) \in \mathbb{R}^{n \times l}$ defined by the backward stochastic differential equation:

$$
\left\{\begin{aligned}
d p(t)= & -\nabla H(t, X(t), u(t), p(t), q(t), r(t, \cdot)) d t \\
& +q(t) d B(t)+\int_{\mathbb{R}^{l}} r\left(t^{-} z\right) \tilde{N}(d t, d z), t \in[0, T) \\
p(T)= & -\nabla g(X(T))
\end{aligned}\right.
$$

We then have the following maximum principle: 
Theorem 10.4. Let $\hat{u} \in \mathcal{V}$ with solution $\hat{X}=X^{(\hat{x})}$ and suppose there exists a triple $(\hat{p}(t), \hat{q}(t), \hat{r}(t, \cdot))$ of the adjoint equation satisfying

$$
E\left[\int_{0}^{T}\left(\hat{X}(t)-X^{(u)}(t)\right)^{T}\left\{\hat{q} \hat{q}^{T}(t)+\int_{\mathbb{R}^{l}} r r^{T}(t, z) \nu(d z)\right\}\left(\hat{X}(t)-X^{(u)}(t)\right) d t\right]<\infty
$$

and

$$
E\left[\int_{0}^{T} \hat{p}(t)^{T}\left\{\sigma \sigma^{T}\left(t, X^{(u)}(t, u(t))\right)+\int_{\mathbb{R}^{l}} \gamma \gamma^{T}\left(t, X^{(u)}(t), u(t), z\right) \nu(d z)\right\} \hat{p}(t) d t\right]<\infty
$$

Moreover, suppose that

$$
H(t, \hat{X}(t), \hat{u}(t), \hat{p}(t), \hat{q}(t), \hat{r}(t, \cdot))=\sup _{v \in U} H(t, \hat{X}(t), v, \hat{p}(t), \hat{q}(t), \hat{r}(t, \cdot))
$$

for all $t$, that $g(x)$ is a concave function of $x$ and that

$$
\hat{H}(x):=\max _{v \in U} H(t, x, v, \hat{p}(t), \hat{q}(t), \hat{r}(t, \cdot))
$$

exists and is a concave function of $x$, for all $t \in[0, T]$.

Then $\hat{u}$ is an optimal control.

Proof. See Theorem 3.4 in Øksendal and Sulem [2000].

\subsection{Dynamic Programing}

We now present the second way of finding the optimal control for dynamic system. As before let $\left(\Omega, \mathcal{F},\{\mathcal{F}\}_{t \geq 0}, P\right)$ be a given filtered probability space satisfying the usual conditions on which a m-dimensional Brownian motion, $B(t)$, is given.

Consider the following stochastic controlled system:

$$
\left\{\begin{array}{l}
d x(t)=b(t, x(t), u(t)) d t+\sigma(t, x(t 9, u(t)) d B(t), \quad t \in[0, T] \\
x(0)=y
\end{array}\right.
$$

with a cost functional

$$
J(s, y ; u(\cdot))=E\left[\int_{0}^{T} f(t, x(t), u(t)) d t+h(x(T))\right] .
$$

Let $U[s, T]$ be the set of all 5 -tuple $\hat{v}(\cdot:=(\Omega, \mathcal{F}, \mathbf{P}, W(\cdot), u(\cdot)) \in \mathcal{U}[0, T]$ satisfying

1. $(\Omega, \mathcal{F}, \mathbf{P})$ is a complete probability space

2. $u(\cdot)$ is measurable and $\mathcal{F}_{t^{-} \text {adapted, }}$

We now have the Stochastic Dynamic Programing Problem:

Problem 10.5. Minimize (10.14) over $\mathfrak{U}[0, T]$. 
For any $(s, y) \in[0, T) \times \mathbb{R}^{n}$ a 5 -tuple $\hat{v}(\cdot:=(\hat{\Omega}, \hat{\mathcal{F}}, \hat{\mathbf{P}}, \hat{W}(\cdot), \hat{u}(\cdot)) \in \mathcal{U}[0, T]$ satisfying

$$
J(\hat{u}(\cdot))=\inf _{u(\cdot) \in \mathcal{U}[0, T]} J(u(\cdot))
$$

is called an optimal control.

Let $\phi(t, x, u):=(b(t, x, u), \sigma(t, x, u), f(t, x, u), h(x))$. Assume , similar to the above requirements, that

1. $(U, d)$ is a Polish space and $0<T<\infty$.

2. the functions $\sigma:[0, T] \times \mathbb{R}^{n} \times U \rightarrow \mathbb{R}^{n \times m}, h: \mathbb{R}^{n} \mapsto \mathbb{R}, b:[0, T] \times \mathbb{R}^{n} \times U \rightarrow \mathbb{R}^{n}$, $f:[0, T] \times \mathbb{R}^{n} \times U \rightarrow \mathbb{R}$ are uniformly continuous, and there exist a constant $L>0$ and a modulus of continuity $\bar{\omega}:[0, \infty) \rightarrow[0, \infty)$ such that

$$
\begin{cases}|\phi(t, x, u)-\phi(t, \hat{x}, \hat{u})| \leq L|x-\hat{x}|+\bar{\omega}(d(u, \hat{u})), & \text { for all } t \in[0, T], x, \hat{x} \in \mathbb{R}^{n}, u, \hat{u} \in U \\ |\phi(t, 0, u)| \leq L, & \text { for all }(t, u) \in[0, T] \times U\end{cases}
$$

Under the above we can define the value function as

$$
\left\{\begin{array}{l}
V(s, y)=\inf _{u(\cdot) \in \mathcal{U}[[s, T]} J(s, y ; u()) ; \text { for all }(s, y) \in[0, T) \times \mathbb{R}^{n} \\
V(t, y)=h(y) ; \text { for all } y \in \mathbb{R}^{n} .
\end{array}\right.
$$

We now have the following version of Bellman's principle of optimality:

Theorem 10.6. Under the above assumptions for any $(s, y) \in[0, T) \times \mathbb{R}^{n}$

$$
V(s, y)=\inf _{u(\cdot) \in \mathcal{U}[[s, T]} E\left[\int_{s}^{\hat{s}} f(t, x(t ; s, y, u(\cdot)), u(t)) d t+V(\hat{s}, x(\hat{s} ; s, y, u(\cdot)))\right],
$$

for all $0 \leq s \leq \hat{s} \leq T$.

Proof. See Theorem 3.3 in Yong and Zhou [1999]

\subsubsection{HJB Equation}

Proposition 10.7. Under the above assumptions and the assumption that the value function $V \in C^{1,2}\left([0, T] \times \mathbb{R}^{n}\right)$. Then $V$ is a solution of the following terminal value problem of a second-order partial differential equation:

$$
\left\{\begin{array}{l}
-v_{t}+\sup _{u \in U} G\left(t, x, u,-v_{x},-v_{x x}\right)=0 ; \text { for all }(t, x) \in[0, T) \times \mathbb{R}^{n}, \\
\left.v\right|_{t=T}=h(x), x \in \mathbb{R}^{n}
\end{array}\right.
$$

where

$$
G(t, x, u, p, P):=\frac{1}{2} \operatorname{tr}\left(P \sigma(t, x, u) \sigma(t, x, u)^{T}\right)+\langle p, b(t, x, u)\rangle-f(t, x, u)
$$

for all $(t, x, u, p, P) \in[0, T] \times \mathbb{R}^{n} \times U \times \mathbb{R}^{n} \times \mathcal{S}^{n}$.

Proof. See Proposition 3.5 in Yong and Zhou [1999] 
The equation (10.15) is called the Hamilton-Jacobi-Bellman equation (HJB equation), G defined by (10.16) is called the generalized Hamiltonian.

\subsubsection{Viscosity Solution}

Often the requirement of a continuous solution is too strong. Then one needs to find a different concept that does the job. In the our case viscosity solutions is what we need.

Definition 10.1 (Viscosity solutions). A locally bounded function $\varphi \in U S C(\overline{\mathcal{S}})$ is called a viscosity subsolution of (2.20)-(2.21) in $\mathcal{S}$ if (2.21) holds and for each $\psi \in C_{0}^{2}(\mathcal{S})$ and each $y_{0} \in \mathcal{S}$ such that $\psi \geq \varphi$ on $\mathcal{S}$ and $\psi\left(y_{0}\right)=\varphi\left(y_{0}\right)$, we have

$$
\max \left\{\inf _{u \in K}\left[A^{u} \psi\left(y_{0}\right)+f\left(y_{0}, u\left(y_{0}\right)\right)\right], g\left(y_{0}\right)-\psi\left(y_{0}\right)\right\} \geq 0
$$

A function $\varphi \in L S C(\overline{\mathcal{S}})$ is called a viscosity supersolution of (2.20)-(2.21) in $\mathcal{S}$ if (2.21) holds and for each $\psi \in C_{0}^{2}(\mathcal{S})$ and each $y_{0} \in \mathcal{S}$ such that $\psi \leq \varphi$ on $\mathcal{S}$ and $\psi\left(y_{0}\right)=\varphi\left(y_{0}\right)$ we have

$$
\max \left\{\inf _{u \in K}\left[A^{u} \psi\left(y_{0}\right)+f\left(y_{0}, u\left(y_{0}\right)\right)\right], g\left(y_{0}\right)-\psi\left(y_{0}\right)\right\} \leq 0 .
$$

Further, if $\varphi \in C\left([0, T] \times \mathbb{R}^{n}\right)$ is both a viscosity subsolution and a viscosity supersolution it is called a viscosity solution.

\subsubsection{Jump Diffusions}

In this section we consider adding a jump part to our diffusion so that the dynamics are given by:

$$
\left\{\begin{array}{l}
d Y(t)=b(Y(t), u(t)) d t+\sigma(Y(t), u(t)) d B(t) \\
+\int_{\mathbb{R}^{k}} \gamma\left(Y\left(t^{-}\right), u\left(t^{-}\right), z\right) \bar{N}(d t, d z) \\
Y(0)=y \in \mathbb{R}^{k}
\end{array}\right.
$$

Let $\mathcal{S} \subset \mathbb{R}^{k}$ be our solvency region and let the cost functional given by:

$$
J(s, y ; u(\cdot))=E\left[\int_{0}^{\tau_{S}} f(t, Y(t), u(t)) d t+g\left(Y\left(\tau_{s}\right)\right) \mathbf{1}_{\left\{\tau_{s}<\infty\right\}}\right] .
$$

We say that a control is admissible and write $u \in \mathcal{V}$ if there is a unique strong solution, $Y(t)$, of (10.19) for all $y \in \mathcal{S}$ and

$$
E\left[\int_{0}^{\tau_{\mathcal{S}}} f^{-}(t, Y(t), u(t)) d t+g^{-}\left(Y\left(\tau_{\mathcal{S}}\right)\right) 1_{\left\{\tau_{\mathcal{S}}<\infty\right\}}\right]<\infty .
$$

This setup gives us the Stochastic Dynamic Programing Problem for Lévy processes:

Problem 10.8. Minimize (10.20) over $\mathcal{V}[0, T]$.

We now have the following analogue to the above Hamiltonian-Jacobi-Bellman theorem, Theorem 3.1 in Sulem and Øksendal [2007]. 
Theorem 10.9 (HJB for Optimal Control of Jump Diffusions). 1. Suppose $\phi \in C^{2}(\mathcal{S}) \cup C(\overline{\mathcal{S}})$ satisfies the following:

(i) $A^{u} \phi(y)+f(y, u) \leq 0$ for all $y \in \mathcal{S}, u \in u$.

(ii) $Y\left(\tau_{\mathcal{S}}\right) \in \partial \mathcal{S}$ a.s. on $\left\{\tau_{\mathcal{S}}<\infty\right\}$ and

$$
\lim _{t \rightarrow \tau_{\S}^{-}}=g\left(Y\left(\tau_{\S}\right) \mathbf{1}_{\left\{\tau_{\S}<\infty\right\}}\right.
$$

a.s., for all $u \in \mathcal{V}$.

(iii)

$$
E^{y}\left[\mid \phi\left(Y(\tau)\left|+\int_{0}^{\tau_{s}}\right| A \phi(Y(t)) \mid d t\right]<\infty\right.
$$

for all $u \in \mathcal{V}$ and all $\tau \in \mathcal{T}$.

(iv) $\left\{\phi^{-}(Y(\tau))\right\}_{\tau \leq \tau_{\mathcal{S}}}$ is uniformly integrable for all $u \in \mathcal{V}$ and $y \in \mathcal{S}$.

Then

$$
\phi(y) \geq \Phi(y) \text { for all } y \in \mathcal{S} \text {. }
$$

2. Moreover, suppose that for each $y \in \mathcal{S}$ there exists $u=\hat{u} \in U$ such that

(v) $A^{\hat{u}(y)} \phi(y)+f(y, \hat{u}(y))=0$ and

(vi) $\left\{\phi^{-}\left(Y^{(\hat{u})}(\tau)\right)\right\}_{\tau \leq \tau_{8}}$ is uniformly integrable and $u^{*}(t):=\hat{u}\left(Y\left(t^{-}\right)\right) \in \mathcal{V}$.

Then $u^{*}$ is an optimal control and

$$
\phi(y)=\Phi(y)=J^{\left(u^{*}\right)}(y) \text { for all } y \in \mathcal{S} .
$$

Proof. See Theorem 3.1 in Sulem and Øksendal [2007].

\subsection{Connection Between the Maximum Principle and Dy- namic Programing}

For simplicity we look at the relationship and interpretation in an economic setting for a deterministic system.

\subsubsection{Relationship for Deterministic Systems}

Consider a deterministic control system where the diffusion is given by:

$$
\left\{\begin{array}{l}
\dot{x}(t)=b(t, x(t), u(t)), \quad t \in[s, T] \\
x(s)=y
\end{array}\right.
$$


and the cost functional:

$$
J(s, y ; u(\cdot))=\int_{s}^{T} f(t, x(t), u(t)) d t+h(x(T)) .
$$

for an initial time and initial state $(s, y) \in[0, T] \times \mathbb{R}^{n}$. We assume that the controller belongs to the domain

$$
\mathcal{V}[s, T]=\{u(\cdot):[s, T] \rightarrow U, u(\cdot) \text { is measurable }\}
$$

Now we have an optimal deterministic control problem, similar to the stochastic problem we encountered above:

Problem 10.10. Minimize (10.20) under (10.21) over $\mathcal{V}[s, T]$.

Define the value function as:

$$
\left\{\begin{array}{l}
V(s, y)=\inf _{u(\cdot) \in \mathcal{V}[0, T]} J(u(\cdot)) ;(s, y) \in[0, T) \times \mathbb{R}^{n} \\
V(T, y)=h(y), y \in \mathbb{R}^{n}
\end{array}\right.
$$

Let $\phi(t, x, u):=(b(t, x, u), f(t, x, u), h(x))$. Then we require that.

1. $(U, d)$ is a separable metric space and $0<T<\infty$.

2. the functions $h: \mathbb{R}^{n} \mapsto \mathbb{R}, b:[0, T] \times \mathbb{R}^{n} \times U \rightarrow \mathbb{R}^{n}, f:[0, T] \times \mathbb{R}^{n} \times U \rightarrow \mathbb{R}$ are uniformly continuous, and there exist a constant $L>0$ and a modulus of continuity $\bar{\omega}:[0, \infty) \rightarrow[0, \infty)$ such that

$$
\begin{cases}|\phi(t, x, u)-\phi(t, \hat{x}, \hat{u})| \leq L|x-\hat{x}|+\bar{\omega}(d(u, \hat{u})), & \text { for all } t \in[0, T], x, \hat{x} \in \mathbb{R}^{n}, u, \hat{u} \in U \\ |\phi(t, 0, u)| \leq L, & \text { for all }(t, u) \in[0, T] \times U\end{cases}
$$

3. The functions $b, f$ and $h$ are $C^{1}$ in $x$, and there exists a modulus of continuity $\bar{\omega}:[0, \infty) \rightarrow$ $[0, \infty)$ such that

$$
\left|\phi_{x}(t, x, u)-\phi_{x}(t, \hat{x}, \hat{u})\right| \leq \bar{\omega}(d(u, \hat{u}))
$$

for all $t \in[0, T], x, \hat{x} \in \mathbb{R}^{n}, u, \hat{u} \in U$.

4. $\left\{\mathcal{F}_{t}\right\}_{t \geq 0}$ is the natural filtration generated by $B(t)$, augmented by all the $\mathbf{P}$-null sets in $\mathcal{F}$. the associated Hamilton-Jacobi-Bellman equation is given as:

$$
\left\{\begin{array}{l}
-v_{t}+\sup _{u \in U} H\left(t, x, u,-v_{x},-v_{x x}\right)=0 ; \text { for all }(t, x) \in[0, T) \times \mathbb{R}^{n}, \\
\left.v\right|_{t=T}=h(x), x \in \mathbb{R}^{n}
\end{array}\right.
$$

where

$$
H(t, x, u, p, P):=\frac{1}{2} \operatorname{tr}\left(P \sigma(t, x, u) \sigma(t, x, u)^{T}\right)+\langle p, b(t, x, u)\rangle-f(t, x, u)
$$


for all $(t, x, u, p, P) \in[0, T] \times \mathbb{R}^{n} \times U \times \mathbb{R}^{n} \times \mathcal{S}^{n}$. For the maximum principle we have the following adjoint system:

$$
\begin{cases}\dot{x}(t) & =H_{p}(t, x(t), u(t), p(t)), \text { a.e.t } \in[s, T] ; \\ \dot{p}(t) & =-H_{x}(t, x(t), u(t), p(t)), \text { a.e.t } \in[s, T] ; \\ x(s) & =y ; \\ P(T) & =-h_{x}(x(T)) ; \\ H(t, x(t), u(t), p(t)) & =\max _{u \in U} H(t, x(t), u, p(t)), \text { a.e.t } \in[s, T]\end{cases}
$$

Theorem 10.11. Let the above requirements hold. If $(\hat{x}(\cdot), \hat{u}(\cdot), p(\cdot))$ is an optimal triple and the value function $V \in C^{1,1}\left([0, T] \times \mathbb{R}^{n}\right)$. Then

$$
\begin{aligned}
V_{t}(t, \operatorname{hat} x(t)) & =H\left(t, \hat{x}(t), \hat{u}(t),-V_{x}(t, \hat{x}(t))\right) \\
& =\max _{u \in U} H\left(t, x(t), u,-V_{x}(t, \hat{x}(t))\right)
\end{aligned}
$$

If $V \in C^{1,2}\left([0, T] \times \mathbb{R}^{n}\right)$ and $V_{t, x}$ is continuous, then

$$
V_{x}(t, \hat{x}(t))=-p(t), \text { for all } t \in[s, T] .
$$

Proof. See Theorem 3.1 in Yong and Zhou [1999].

\subsubsection{Economic Interpretation}

From the above Theorem 10.11 we see that a change in $x$ direction at the optimal point $\hat{x}$ is given by $-p x$. So $p$ measures the rate at which the best performance increases. Thus we call $p$ the marginal value of the resource or the shadow price. If the firm purchase the resource in the marked, $p$ represents the maximum price that is economically defendable to pay.

\subsection{Singular Control}

Let $\kappa: \mathbb{R}^{k} \rightarrow \mathbb{R}^{k \times p}$ and $\theta: \mathbb{R}^{k} \rightarrow \mathbb{R}^{p}$ be given continuous functions and assume the state $Y(t)$ is given by the dynamics

$$
\left\{\begin{array}{l}
d Y(t)=b(Y(t), u(t)) d t+\sigma(Y(t), u(t)) d B(t) \\
+\int_{\mathbb{R}^{k}} \gamma\left(Y\left(t^{-}\right), u\left(t^{-}\right), z\right) \bar{N}(d t, d z)+\kappa\left(Y\left(t^{-}\right)\right) d \xi(t) \\
Y\left(0^{-}\right)=y \in \mathbb{R}^{k}
\end{array}\right.
$$

We let $\xi(t) \in \mathbb{R}^{p}$ be an adapted cádlág finite variation process with increasing components and $\xi\left(0^{-}\right)=9$. Since $\xi(t)$ may be singular with respect to the Lebesgue measure $t$, we call $\xi$ a singular control. The process $u(t)$ is a adapted cádlág process with values in a set $U$ and is an absolutely continuous control. Let $\mathcal{S} \subset \mathbb{R}^{k}$ be our solvency region and let the cost functional given by:

$$
J(s, y ; u(\cdot))=E\left[\int_{0}^{\tau_{\S}} f(t, Y(t), u(t)) d t+g\left(Y\left(\tau_{\S}\right)\right) \mathbf{1}_{\left\{\tau_{\delta}<\infty\right\}}\right] .
$$


where

$$
\tau_{\mathcal{S}}=\inf \left\{t>0 ; Y^{u, \xi}(t) \notin \mathcal{S}\right\} \leq \infty
$$

Problem 10.12. Find a value function $\Phi(y)$ and an optimal control $\left(u^{*}, \xi^{*}\right) \in \mathcal{A}$ such that

$$
\Phi(y)=\sup _{(u, \xi) \in \mathcal{A}} J^{u, \xi}(y)=J^{u^{*}, \xi^{*}}(y)
$$

\section{Theorem 10.13.}

1. Suppose there exists a function $\phi \in C^{2}\left(\mathcal{S}^{0}\right) \cup C\left(\mathbb{R}^{k}\right)$ such that

(i) $A^{v} \phi(y)+f(y, v) \leq 0$ for all (constant) $v \in U$ and $y \in \mathcal{S}$;

(ii)

$$
\sum_{i=1}^{k} \kappa_{i j}(y) \frac{\partial \phi}{\partial y_{i}}(y)+\theta_{j}(y) \leq 0
$$

for all $y \in \mathcal{S}, j=1, \ldots, p$;

(iii)

$$
\begin{aligned}
& E^{y}\left[\int _ { 0 } ^ { \tau _ { \S } } \left\{\left|\sigma^{T}(Y(t), u(t)) \nabla \phi(Y(t))\right|^{2}\right.\right. \\
& \left.\left.\left.+\sum_{k=1}^{l} \int_{\mathbb{R}} \mid \phi\left(Y(t)+\gamma^{(k)}(Y(t), u(t), z)\right)-\phi(t)\right)\left.\right|^{2} \nu_{k}(d z)\right\} d t\right]<\infty
\end{aligned}
$$

for all $(u, \xi) \in \mathcal{A}$;

(iv) $\lim _{t \rightarrow \tau_{s}^{-}} \phi(Y(t))=g\left(Y\left(\tau_{s}\right) \mathbf{1}_{\left\{\tau_{s}<\infty\right\}}\right.$ a.s. for all $(u, \xi) \in \mathcal{A}$;

(v) $\left\{\phi^{-}(Y(\tau))\right\}_{\tau \leq \tau_{\delta}}$ is uniformly integrable for all $(u, \xi) \in \mathcal{A}, y \in \mathcal{S}$;

Then

$$
\phi(y) \geq \Phi(y) \text { for all } y \in \mathcal{S} \text {. }
$$

2. Define the nonintervention region $D$ by

$$
D=\left\{y \in S ; \max _{1 \leq j \leq p}\left\{\sum_{i=1}^{k} \kappa_{i j}(y) \frac{\partial \phi}{\partial y_{i}}(y)+\theta_{j}(y)\right\}<0\right\} .
$$

Suppose further that for all $y \in \bar{D}$ there exists $v=\hat{u}(y)$ such that

(vi) $A^{v} \phi(y)+f(y, v)=0$ Moreover, suppose there exists $\hat{\xi}$ such that $(u, \xi) \in \mathcal{A}$ and (vii) $Y^{\hat{u}, \hat{\xi}} \in \bar{D}$ for all $t$; 
(viii)

$$
\sum_{j=1}^{p}\left\{\sum_{i=1}^{k} \kappa_{i j}\left(Y\left(t^{-}\right)\right) \frac{\partial \phi}{\partial y_{i}}\left(Y\left(t^{-}\right)\right)+\theta_{j}\left(Y\left(t^{-}\right)\right)\right\} d \hat{\xi}_{j}^{c}=0
$$

for all $t, 1 \leq j \leq p$, where $\xi^{c}$ is the continuous part of $\eta$.

(ix) $\Delta_{\hat{\xi}} \phi\left(Y\left(t_{n}\right)\right)+\sum_{j=1}^{p} \theta_{j}\left(Y\left(t_{n}^{-}\right)\right) \Delta \hat{\xi}\left(t_{n}\right)=0$ for all jumping times $t_{n}$ of $\hat{\xi}$ and

(x)

$$
\lim _{R \rightarrow \infty} E^{y}\left[\phi\left(Y^{\hat{u}, \hat{\xi}}\left(T_{R}\right)\right)\right]=E^{y}\left[g\left(Y^{\hat{u}, \hat{\xi}}\left(\tau_{\mathcal{S}}\right) \mathbf{1}_{\tau_{\mathcal{S}}<\infty}\right)\right]
$$

where $T_{R}:=\min \left(\tau_{\mathcal{S}}, R\right)$ for all $R<\infty$.

Then

$$
\phi(y)=\Phi(y)
$$

and

$$
(\hat{u}, \hat{\xi})
$$

is an optimal control.

Proof. See Theorem 5.2 in Øksendal and Sulem [2000]. 


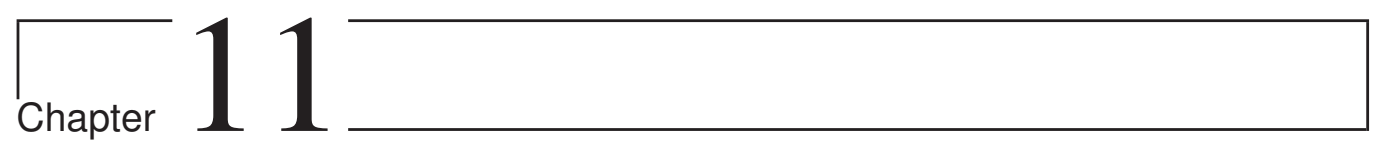

\section{Optimal Stopping}

In addition to the problem of finding an optimal control we often encounter the optimization problem where we need to find the optimal time to stop a dynamic system.

This chapter is based on Sulem and Øksendal [2007].

\subsection{Problem Formulation}

Let us start with a given open set $\mathcal{S} \subset \mathbb{R}^{k}$ (the solvency region) and let $Y(t)$ be a jump diffusion on $\mathbb{R}^{k}$ given by

$$
\begin{cases}d Y(t) & =b(Y(t)) d t+\sigma(Y(t)) d B(t)+\int_{\mathbb{R}^{k}} \gamma\left(Y\left(t^{-}\right), z\right) \bar{N}(d t, d z) \\ Y(0) & =y \in \mathbb{R}^{k}\end{cases}
$$

where $b: \mathbb{R}^{k} \rightarrow \mathbb{R}^{k}, \sigma: \mathbb{R}^{k} \rightarrow \mathbb{R}^{k \times m}$ and $\gamma: \mathbb{R}^{k} \times \mathbb{R}^{k} \rightarrow \mathbb{R}^{k \times l}$ are functions such that a unique solution $Y(t)$ exists. Let

$$
\tau_{\mathcal{S}}=\inf \{t>0 ; Y(t) \notin \mathcal{S}\}
$$

be the bankruptcy time and let $\mathcal{T}$ denote the set of all stopping times $\tau \leq \mathcal{S}$. For a continuous functions $f: \mathbb{R}^{k} \rightarrow \mathbb{R}$ and $g: \mathbb{R}^{k} \rightarrow \mathbb{R}$ such that

$$
E^{y}\left[\int_{0}^{\tau_{s}} f^{-}(Y(t)) d t\right]<\infty
$$

for all $y \in \mathcal{R}^{k}$ and such that the family $\left\{f^{-}(Y(\tau)) \mathbf{1}_{\{\tau<\infty\}}, \tau \in \mathcal{T}\right\}$. Define the performance criterion as

$$
J^{\tau}(y)=E^{y}\left[\int_{0}^{\tau} f(Y(t)) d t+g(Y(\tau)) \mathbf{1}_{\{\tau<\infty\}}\right] .
$$

Then we have the following problem: 
Problem 11.1. Find $\Phi(y)$ and $\tau^{*} \in \mathcal{T}$ such that

$$
\Phi(y):=\sup _{\tau \in \mathcal{T}} J^{\tau}(y)=J^{\tau^{*}}(y), y \in \mathbb{R}^{k} .
$$

\subsection{Sufficient Optimal Stopping Theorem}

We are now able to present some sufficient conditions for that a function $\phi$ is the value function $\Phi$ and that the corresponding stopping time $\tau_{\mathcal{D}}$ is optimal. Let $\overline{\mathcal{S}}$ be the topological closure of $\mathcal{S}$.

Theorem 11.2 (Integrovariational Inequalities for Optimal Stopping).

1. Suppose we can find a function $\phi: \mathcal{S} \rightarrow \mathbb{R}$ such that

(a) $\phi \in C^{1}(\mathcal{S}) \cup C(\overline{\mathcal{S}})$;

(b) $\phi \geq g$ on $S$; Define

$$
D=\{y \in \mathcal{S} ; \phi(y)>g(y)\}
$$

and suppose

(c) $E^{y}\left[\int_{0}^{\tau_{s}} \mathbf{1}_{\partial D}(Y(t)) d t\right]=0$;

(d) $\partial D$ is a Lipschitz surface;

(e) $\phi \in C^{2}(\mathcal{S} \backslash \partial D)$ with locally bounded derivative near $\partial D$;

(f) $A \phi+f \leq 0$ on $\mathcal{S} \backslash \partial D$;

(g) $Y\left(\tau_{\mathcal{S}}\right) \in \partial \mathcal{S}$ a.s. on $\left\{\tau_{\mathcal{S}}<\infty\right\}$ and $\lim _{t \rightarrow \tau_{\mathcal{S}}} \phi(Y(t))=g\left(Y\left(\tau_{\mathcal{S}}\right)\right)$;

(h) $E^{y}\left[|\phi(Y(\tau))|+\int_{0}^{\tau_{s}}|A \phi(Y(t))| d t\right]<\infty$ for all $\tau \in \mathcal{T}$;

Then $\phi(y) \geq \Phi(y)$ for all $y \in \overline{\mathcal{S}}$.

2. Moreover, if

(a) $A \phi+f=0$ on $D$;

(b) $\tau_{\mathcal{D}}:=\inf \{t>0, Y(t) \notin D\}<\infty$ a.s. for all $y$;

(c) $\left\{\phi(Y(\tau)), \tau \in \mathcal{T}, \tau \leq \tau_{D}\right\}$ is uniformly integrable, for all $y$.

Then

$$
\phi(y)=\Phi(y)
$$

and

$$
\tau^{*}=\tau_{D}
$$

is an optimal stopping time.

Proof. See theorem 2.2 in Sulem and Øksendal [2007]. 


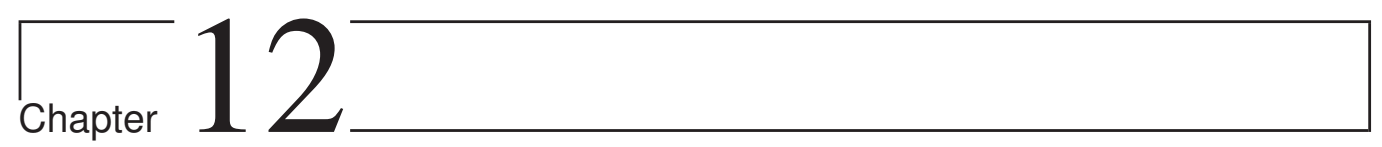

\section{Risk Measures}

Controlling risks is of great value and interest. To be able to control the given risks we need to give it a proper mathematical formulation. We first start with a risk measure, value at risk, that is not constructed around a properly given framework. It is as we will see not a good way of measuring risks. After presenting this risk measure, we give a proper axiomatic definition of a risk measure.

This chapter is based on Haadem [2009].

\subsection{Value at Risk}

Value at Risk (VaR) is defined as follows:

$$
\operatorname{VaR}^{\alpha}(L)=-\inf \{x \in \mathbb{R}: P[L \leq x]>\alpha\}
$$

VaR is widely used in the industry but is heavily criticized for several reasons. Firstly, VaR is completely ignorant of the seriousness of the worst cases, which could create a false sense of security. By investing in asymmetric positions that generate small gains and very rarely have losses, VaR could be constructed to underestimate the risk. An example is the credit-default swap that generates steady income. If the probability of default is less than $1 \%, 99 \%$ Var is 0 , but on the offset of a default a substantial loss could be incurred. Second, a very critical flaw is that VaR can generate scenarios where risk is decreased under decentralization.VaR is not subadditive. That means the VaR of a combined portfolio can be larger than the sum of the VaRs of its components. In the Jorion-Taleb debate, Jorion and Taleb [1997] argued about VaR. Taleb claimed that VaR:

1. Is charlatanism because it claimed to estimate the risks of rare events, which is impossible.

2. Gives false confidence.

3. Could be exploited by traders.

In Brown and Einhorn [2008], David Einhorn also claims that VaR

1. Is potentially catastrophic, as it can create a false sense of security among executives and regulators.

2. Leads to excessive risk-taking and use of leverage.

3. Created an incentive to take remote but excessive risk.

4. Focuses on the manageable risks near the center of the distribution and ignored the tails. 


\subsection{Coherent Risk Measures}

To overcome these shortcomings, an axiomatic approach to risk measure has been a key point for development in risk management and mathematical finance in the recent years. The concept of coherent measures and more generally convex measures follows an axiomatic approach and are now well developed. Initial research into constructing a solid groundwork for risk measures was initiated by Artzner, Delbaen, Eber, and Heath. Artzner and Delbaen provided the pathbreaking axiomatic definition of coherent risk measures in the papers Artzner et al. [1997] and Artzner et al. [1999]. They provided four axioms for a coherent risk measure,

(I) Translation invariance. For all $x \in X$ and all real numbers $\alpha$,

$$
\rho(x+\alpha)=\rho(x)-\alpha,
$$

(II) Sub additivity. For all $x_{1}$ and $x_{2} \in X$,

$$
\rho\left(x_{1}+x_{2}\right) \leq \rho\left(x_{1}\right)+\rho\left(x_{2}\right),
$$

(III) Positive homogeneity. For all $\lambda \geq 0$ and all $x \in X$,

$$
\rho(\lambda x)=\lambda \rho(x)
$$

(IV) Monotonicity. For all $x$ and $y \in X^{\prime}$ with $x \leq y$,

$$
\rho(y) \leq \rho(x)
$$

In financial theory hedging and pricing of claims has been given much attention. In a complete market, if the payoff of a claim can be constructed from the payoff of basis assets, this value is uniquely determined and other prices would lead to an arbitrage opportunity. Whenever this perfect replication is unattainable, due to market frictions, transaction costs and sources of unhedgeable risk, we get an incomplete market. In an incomplete market, the standard BlackScholes pricing methodology fails because the price of the focus asset is no longer unique. This can be solved by the representative agent equilibrium, where the pricing functional is obtained from the marginal utility of the optimized representative agent's consumption. Another way to solve this problem is to construct good deal bounds so that the price of a non-redundant contingent claim is not unique. As Černý and Hodges [2000] points out, no arbitrage is a rather weak requirement as can be seen in their example of a claim with zero price that either earns 1000 or loses 1 with equal probability. Good deals are defined as an opportunity to buy a desirable claim at no cost. To construct a good deal bound given a risk measure, one hedges the given claim with a portfolio of self-financed, basic assets so to maximize w.r.t. the given risk measure. Then one remove prices that give an undesirable good hedging strategy. This provides a price interval for every contingent claim called generalized arbitrage bounds or good deal bounds by Černý and Hodges [2000]. The connection to coherent risk measures were made by Jaschke and Küchler [2001], who proved that coherent risk measures are essentially equivalent to generalized arbitrage bounds.

A valuable property for risk measure is that they have a close relation to utility function and asset pricing. If we consider the classical $(\mu, \sigma)$ portfolio optimization theory of Markowitz we can use $(\mu, \rho)$, where $\rho$ is coherent risk measure. This enables us to consider the $(\mu, \rho)$ problem, which can be viewed as the problem of maximizing $U=\mu-\lambda \rho$. When the preference function $\Phi(X)=E[U(X)]$, where $\mathrm{U}$ denotes the utility function specific to each decision maker, 
do not separate risk or value, Jia and Dyer [1996] proved that it is possible to derive an explicit risk measures: $\rho(X)=-E[U(X-E(X))]$. So from the connection to utility functions and good deal bounds, we can conclude that coherent risk measures are consistent with economic theories of arbitrage as well as utility maximization.

The sub linearity axiom of coherent risk measures gives us that $\rho(\lambda X) \leq \lambda \rho(X)$ but we may want to model cases where a single position $(\lambda X)$ could be less liquid, and therefore more risky, than that of $\lambda$ smaller positions, so convex risk measure was later extended to convex risk measures by Füllmer and Schied [2002] and Frittelli and Gianin [2002]. Although these new risk measures are very useful in risk management, they are static. Static risk measures only quantify risk at a single instance in the future and was by Föllmer and Leukert [1999] generalized to a dynamic setting. To construct a dynamic risk measure Riedel [2003] considered the changes of a position and availability of new information with time. As additional information about the position may be released with time and changes may occur in the position or there may be an intermediate cash flow, there may be a need for a reassessment of the position under this new information. Changes in the position are to be taken into account by recalculating the (stochastic) present value of future positions. Information is processed in a Bayesian way on a set of generalized scenarios. Dynamic risk measures allow a manager the flexibility of adjusting a position. They take a random cash flow and return a random process as a function of time.

We will now review the axioms for coherent risk measures in depth.

\subsection{An Axiomatic Approach}

Capturing reality necessitates a minimum set of requirements or proposition we consider selfevident. This has been the starting point for every mathematical approach to model reality since Euclid. For financial risk, capturing these propositions has been an area of neglect. Concepts have been intuitively developed as they seem to give a logical measure of risk but no formal requirement has been given. These intuitions need to be made concrete and unambiguous. To answer this challenge Artzner et al. [1997] proposed a set of axioms that a risk measure needs to fulfill;

Axiom 12.1 (Translation invariance). For all $x \in X$ and all real numbers $\alpha$,

$$
\rho(x+\alpha r)=\rho(x)-\alpha .
$$

This axiom, translation invariance, ensures that risk measures are given in the same units as the final net worth. We see that by adding a sure return $m$ to a position $X$ the risk $\rho(X)$ decrease by $m$. The next axiom is probably the one that seems most intuitive

Axiom 12.2 (Sub additivity). For all $x_{1}$ and $x_{2} \in X$,

$$
\rho\left(x_{1}+x_{2}\right) \leq \rho\left(x_{1}\right)+\rho\left(x_{2}\right) .
$$

One would naturally think that if two independent financial institutions that separately have adequate reserves to cover extreme scenarios would be in no greater risk after a merger. Therefore the risk of a portfolio should be no more than the sum of its components. This is where VaR often fails. It is coherent in the case of unimodal distributions like the normal- and t-distribution. A perfectly diversified portfolio, due to the Central Limit theorem, is normal distributed. Most portfolios are not perfectly diversified and have significant deviation from the normal distribution. Diversification risk should be monitored as they can lead to inadequate capital reserves and unexpected losses. Market risk factors such as equity indices, foreign exchange rates, commodity prices and interest rates are continuously distributed. They exhibit properties of skewness and excess kurtosis so they are not normally distributed. Many market risk return distributions are not normally distributed but they are very often unimodal so 
that VaR is coherent. Credit rating migrations and insurance risk are typical cases where the return distributions are not unimodal and VaR is not coherent. In this case VaR can be constructed to artificially lower risk. This is mainly due to the large losses of low-probability events.

The above axiom gives us that $\rho(\lambda x) \leq \lambda \rho(x)$, but we should not be able to lower the risk of multiple identical portfolios by merging them.

Axiom 12.3 (Positive homogeneity). For all $\lambda \geq 0$ and all $x \in X$,

$$
\rho(\lambda x)=\lambda \rho(x)
$$

This axiom ensures us that multiplying a position multiply the risk by the same amount. Another intuition we have about risk measures is that it seems clear that when a position is surely larger than another position, the risk of the first should be lower than the risk of the last.

Axiom 12.4 (Monotonicity). For all $x$ and $y \in X^{\prime}$ with $x \leq y$,

$$
\rho(y) \leq \rho(x)
$$

Remark 12.5. The axioms are motivated from a supervising agency's point of view. From this perspective a risk measure is viewed as capital requirement, the amount needed to make the position acceptable.

We can now define a coherent risk measure.

Definition 12.6. A coherent risk measure is a functional $\rho: X \longmapsto \Re$, that satisfies axioms 1.1 .1 - 1.1.4.

Definition 12.7. We say a risk measure is continuous from below (resp. above) if for any increasing (resp. decreasing) sequence $X_{n}$ of elements of $L^{\infty}$ such that $X=\lim X_{n}$ a.s., the sequence $\rho\left(X_{n}\right)$ has the limit $\rho(X)$ a.s.

In Pedersen [1995] (2.3.1) we find the following definition;

Definition 12.8. Let $X$ be a vector space. A Minkowski functional on $X$ is a function $\rho: X \rightarrow$ $\mathbb{R}$ such that

(a) $\rho(x+y) \leq \rho(x)+\rho(y), x, y \in X$

(b) $\rho(\alpha x)=\alpha \rho(x), x \in X, \alpha \geq 0$,

We can see that a coherent risk measure is a Minkowski functional (a semi-norm if $F=\mathbb{R}$ ).

In most cases there will be no loss of generality by assuming $\rho$ is normalized in the sense that

$$
\rho(0)=0 .
$$

If $\rho(x)$ is $\leq 0$ we say the position is acceptable, if not $\rho(x)$ is the amount that must be added to the position to make it acceptable.

Remark 12.9. It can be, by definition of axioms, argued that the stated axioms are not correct ones, but they are nevertheless required as basis for a thorough mathematical approach.

It can be shown that every coherent risk measure can be represented in a specific form.

Theorem 12.10. [Representation theorem Föllmer and Penner [2006b]] A functional $\rho: X^{\prime} \rightarrow$ $\mathbb{R}$ is a coherent risk measure iff there exists a closed convex set $\mathcal{Q}$ of P-absolutely continuous probability measures on a set of states of nature, such that

$$
\rho(x)=\sup _{\mathcal{Q}} E_{Q}[-x], \forall x \in L^{\infty}
$$

This tells us that any coherent risk measure can be represented as the supremum of the expected loss over a set of scenarios. 


\subsubsection{Axioms on Acceptance Sets}

We have discussed the axioms that a risk measure should arguably satisfy. We will now review the related concept of defining an acceptable position through acceptance sets. As we will see later there is a strong correlation between these two approaches.

Definition 12.11. We let $\mathcal{A}_{i, j}, j \in J_{i}$ be a set of final net worth, in currency $i$, which is accepted by regulator $j$.

We now state some axioms on acceptance sets;

Axiom 12.12. The Acceptance set $\mathcal{A}$ contains $X_{+}^{\prime}$.

Axiom 12.13. The Acceptance set $\mathcal{A}$ do not intersect the set $X_{--}^{\prime}$ where

$$
X_{--}^{\prime}=\{X \mid \text { for each } x \in X, X(x)<0\}
$$

The meaning of these two axioms is that a non-negative net worth do not require additional capital to be acceptable. On the other hand a strictly negative one needs additional security.

Axiom 12.14. The acceptance set $\mathcal{A}$ is convex.

This captures the risk aversion of the regulator, exchange director or trading room supervisor. Artzner,Eber and Heath also suggest the less natural requirement of

Axiom 12.15. The acceptance set $\mathcal{A}$ is a positively homogeneous cone.

The correlation between risk measures and acceptance sets will be made clear by using the two definitions given below.

Definition 12.16 (Risk measure associated with an acceptance set). The risk measure associated with the acceptance set $\mathcal{A}$ is the mapping from $X$ into $\mathbb{R}$ defined by

$$
\rho_{\mathcal{A}}(x)=\inf \{m \mid m+x \in \mathcal{A}\} .
$$

Definition 12.17 (Acceptance set associated with a risk measure). The acceptance set associated with a risk measure $\rho$ is the set

$$
\mathcal{A}_{\rho}=\{x \in X \mid \rho(x) \leq 0\}
$$

The next theorem given by Artzner et al. [1997], links acceptance sets to risk measures.

Theorem 12.18. If the set $\mathcal{A}$ satisfy the four axioms 12.12-12.15, the risk measure $\rho_{\mathcal{A}}$ is coherent. Moreover $\mathcal{A}_{\rho_{\mathcal{A}}}=\overline{\mathcal{A}}$.

Earlier we gave a representation theorem for coherent risk measures. This representation has a natural connection to acceptance sets, as shown by Delbaen [2002].

Theorem 12.19. Let $\rho: L^{\infty}(\Sigma, \mathcal{F}, P) \rightarrow \mathbb{R}$ be a coherent risk measure. Then there exist a closed convex set $\mathcal{P}$ of $P$-absolutely continuous probability measures such that

$$
\begin{aligned}
& \rho(x)=\sup _{\mathcal{Q} \in \mathcal{P}} E_{Q}[-x], \forall x \in L^{\infty} \\
& \Leftrightarrow
\end{aligned}
$$

the acceptance set $\left\{x \in L^{\infty}: \rho(x) \leq 0\right\}$ is a $\sigma\left(L^{\infty}, L^{1}\right)$ - closed convex cone

This thorough approach is a breakthrough to risk management and provides a mathematical understanding of risk. When coherent risk measure was first introduced in 1997 it created a heated debate since VaR, which had a strong position amongst regulators and practitioners, was seen to not be coherent and not even convex (see below). 


\subsection{A Generalization to Convex Risk Measures}

By looking at liquidity issues for large portfolios or bonds issued by firms as opposed to treasury bonds or bills, Frittelli and Gianin [2002] argues against the requirement for sub-additivity and positive homogeneity. There could be liquidity risks if an investor holds a portfolio that is large relative to the marked depth. If the market is not able to absorb a sudden sell-off of a large position, doubling the investment in the position increases the risk by more than the double. To account for this the concept of convex risk measures was proposed by Föllmer and Penner [2006a]. Heath considered risk measures in finite settings which was later extended to the infinite case by Föllmer and Schied [2002] and Frittelli and Gianin [2002]. They propose to relax the conditions of positive homogeneity and of sub additivity. They required instead;

Axiom 12.20. Convexity. $\rho(\lambda x+(1-\lambda) y) \leq \lambda \rho(x)+(1-\lambda) \rho(y)$ for any $\lambda \in[0,1]$.

This axiom ensures that the risk is not increased by diversification of the portfolio.

Definition 12.21. A map $\pi: X^{\prime} \longrightarrow \mathbb{R}$ will be called a convex measure of risk if it satisfies the condition of: translation invariance (axiom 12.1), monotonicity (axiom 12.4), and convexity (axiom 12.20).

Let the risk measure, $\pi$, be normalized, meaning $\pi(0)=0$. Then we can see that the axiom of convexity gives

$$
\begin{aligned}
& \pi(\delta x) \leq \delta \pi(x), \forall \delta \in[0,1], \forall x \in X \\
& \pi(\delta x) \geq \delta \pi(x), \forall \delta \geq 1, \forall x \in X
\end{aligned}
$$

In light of the liquidity discussion these two axioms seem reasonable since large portfolios will suffer from liquidity issues. The convexity requirement encourages diversification of risk, which is in coherence with our understanding of portfolio management.

As we showed, coherent risk measures have a representation form as the supremum of the expected loss. It turns out that this representation depends only on the sub-linearity axiom. Frittelli and Gianin [2002] gives a representation for the larger class of convex risk measures.

Definition 12.22. Let $\pi: X \mapsto \mathbb{R}$. If it exists a convex functional $F: X^{\prime} \mapsto \mathbb{R} \cup+\infty$ satisfying $\inf _{x^{\prime} \in X^{\prime}} F\left(x^{\prime}\right)=0$ such that

$$
\pi(x)=\sup _{x^{\prime} \in \mathcal{P}}\left\{x^{\prime}(x)-F\left(x^{\prime}\right)\right\}<+\infty, \text { for all } x \in X,
$$

where $\mathcal{P}=\left\{x^{\prime} \in X^{\prime}: F\left(x^{\prime}\right)<\infty\right\}$ is the effective domain of $F$. Then we say that $\pi$ is representable or that $\rho(x)=\pi(-x)$ is a convex risk measure.

Theorem 12.23 (Frittelli and Gianin [2002]). 1. A functional $\pi: X \mapsto \mathbb{R}$ is representable if and only if it is convex and lower semi-continuous.

2. A functional $\pi: X \mapsto \mathbb{R}$ is representable with $F=0$ on $\mathcal{P}$ if and only if it is sublinear and lower semi-continuous.

The financial interpretation is that $F$ represents a correction term and $x^{\prime}(x)$ the expected loss, $\rho$ is the supremum over a set of generalized scenarios where the correction term is dependent on the scenario. 
One type of penalty function $F(\mathcal{Q})$ is the relative entropy of $\mathcal{Q}$ with respect to $\mathcal{P}$, defined as

$$
F(\mathcal{Q}) \equiv I(\mathcal{Q} ; \mathcal{P}) \equiv E_{\mathcal{Q}}\left[\log \frac{d \mathcal{Q}}{d \mathcal{P}}\right]=E\left[\frac{d \mathcal{Q}}{d \mathcal{P}} \log \frac{d \mathcal{Q}}{d \mathcal{P}}\right] \text {. }
$$

See Grandits and Rheinlander [2002]. Entropy is not a metric because $d(p, q) \neq d(q, p)$ (but $d(p, q) \geq 0, d(p, q)=0$ iff $p=q$.$) . Relative entropy, also called the Kullback-Leibler, gives the$ proximity of two measures. In Cont and Tankov [2004] we see that if a measure are generated by an exponential Lévy model, the relative entropy can be expressed in terms of Lévy measures:

Proposition 12.1. Let $P$ and $Q$ be two equivalent measures on $(\Omega, F)$ generated by an exponential Lévy model with Lévy triplet $\left(\sigma^{2}, \nu^{P}, \gamma^{P}\right)$ and $\left(\sigma^{2}, \nu^{Q}, \gamma^{Q}\right)$. Assume $\sigma>0$. The relative entropy $\xi(Q, P)$ is given by

$$
\begin{aligned}
\xi(Q, P)= & \frac{T}{2 \sigma^{2}}\left\{\gamma^{Q}-\gamma^{P}-\int_{-1}^{1} x\left(\nu^{Q}-\nu^{P}\right)(d x)\right\}^{2} \\
& +T \int_{-\infty}^{\infty}\left(\frac{d \nu^{Q}}{d \nu^{P}} \log \frac{d \nu^{Q}}{d \nu^{P}}+1-\frac{d \nu^{Q}}{d \nu^{P}}\right) \nu^{P}(d x) .
\end{aligned}
$$

We can then see that the first term penalizes the difference in drifts while the second one penalizes the difference in Lévy measures.

Another example is the quadratic distance:

$$
E\left[\left(\frac{d Q}{d P}\right)^{2}\right]
$$

In Föllmer and Schied [2002] theorem 4.12 we have that the penalty function have a given representation.

Theorem 12.24. Any convex risk measure on $X$ is on the form

$$
\rho(x)=\sup _{Q \in M_{a}} E_{Q}[-x]-F_{\min }(Q),
$$

where $M_{a}$ as above and the penalty function $F_{\min }$ is given by

$$
F_{\min }:=\sup _{x \in \mathcal{A}_{\rho}} E_{Q}[-x]
$$

Moreover, $F_{\min }(Q)$ is the minimal penalty function for $\rho$, i.e. for any penalty function $F$, $F(Q) \geq F_{\min }(Q)$ for all $Q \in M_{a}$.

This functional representation has a connection to pricing functionals in incomplete markets. For each non-attainable claim $x \in X$ there is an interval of prices that gives absence of arbitrage. The maximum price $\hat{x}$ in this interval is given by

$$
\hat{x}=\sup _{\mathcal{P}^{\prime}} E_{\mathcal{P}^{\prime}}[x],
$$

see Lüthi and Doege [2005].

Remark 12.25. As pointed out by Acerbi [2004], the issue of designing a convex measure that allow for sub additive violations solely due to liquidity is very difficult, and if the measure 
is allowed to break the sub additivity in general cases, not just to model liquidity, there is a possibility for loss effect.

\section{5 g-Brownian Motion and Dynamic Risk Measures}

The risk measures discussed all quantify risk at a single point in the future. They are static risk measures. Most investors are making portfolio decisions dynamically and usually at discrete times. As a consequence Föllmer and Leukert [1999] came up with the concept of dynamic risk measures. Important research and development of dynamic risk measure include; Peng [1997], Peng [2003], Frittelli and Gianin [2004] and Föllmer and Penner [2006b]. We now define a dynamic (convex or not) risk measure, $\left(\rho_{t}\right)_{t \in A}$, where A is not necessarily countable, and the set of $\rho_{t}$ is a net. At an instance $t \in A, \rho_{t}$ represent the riskiness of our position at time t. We also need the boundary requirement that $\rho_{0}$ is a static risk measure.

Definition 12.26 (Gianin [2002a] and Gianin [2002b]). A dynamic risk measure is a net $\left(\rho_{t}\right)_{t \in A}$ such that

- $\rho_{t}: L^{p}\left(\mathcal{F}_{t}\right) \rightarrow L^{0}\left(\Sigma, \mathcal{F}_{t}, P\right)$, for all $t$.

- $\rho_{0}$ is a static risk measure.

- $\rho_{T}(x)=-x$ P-a.s. for all $x \in X^{\prime}$

As before we will continue the axiomatic approach by listing some desirable properties for $\left(\rho_{t}\right)_{t \in T}$.

Axiom 12.27. Convexity: $\rho_{t}$ is convex for all $t \in[0, T] P$-a.s.

Axiom 12.28. Positivity: $x \geq 0 \Rightarrow \forall t \in[0, T], \rho_{t}(x) \leq \rho_{t}(0)$ P-a.s.

Axiom 12.29. monotonicity: $x \geq y \Rightarrow \forall t \in[0, T], \rho_{t}(x) \leq \rho_{t}(y)$ P-a.s.

Axiom 12.30. Sub-additivity: $\forall x, y \in L^{p}\left(\mathcal{F}_{t}\right), \forall t \in[0, T] \rho_{t}(x+y) \leq \rho_{t}(x)+\rho_{t}(y) P$-a.s.

Axiom 12.31. Positive homogeneity: $\forall \alpha \geq 0, \forall x \in L^{p}\left(\mathcal{F}_{t}\right), \forall t \in[0, T] \rho_{t}(\alpha x)=\alpha \rho_{t}(x)$ P-a.s.

Axiom 12.32. Translation-invariance: $\forall t \in[0, T], \forall \mathcal{F}_{t}$-measurable $a \in L^{p}\left(\mathcal{F}_{t}\right), \forall x \in L^{p}\left(\mathcal{F}_{t}\right)$ $\rho_{t}(x+a) \leq \rho_{t}(x)-a P$-a.s.

Axiom 12.33. Constancy: $\forall c \in \mathbb{R}, \forall t \in[0, T] \rho_{t}(c)=-c$ P-a.s.

Definition 12.34 (Frittelli and Gianin [2002]). Let us now define convex and coherent risk measures respectively as;

1. A dynamic risk measure, $\left(\rho_{t}\right)_{t \in T}$, is called coherent if it satisfy the axiom of positivity, sub-additivity, positive homogeneity and translation invariance.

2. A dynamic risk measure, $\left(\rho_{t}\right)_{t \in T}$, is called convex if it satisfy the axiom of convexity and $\rho_{t}(0)=0$.

Definition 12.35. Two important properties of dynamic risk measures are;

1. $\left(\rho_{t}\right)_{t \in T}$ is said to be time consistent if

$$
\rho_{0}\left[x \mathbf{1}_{A}\right]=\rho_{0}\left[-\rho_{t}(x) \mathbf{1}_{A}\right], \forall t \in[0, T], \forall x \in L^{p}\left(\mathcal{F}_{t}\right), \forall A \in \mathcal{F}_{t} .
$$

2. A dynamic risk measure is continuous from below (resp. above) if each $\rho_{t}$ is continuous from below (resp. above). 


\subsubsection{Dynamic Risk Measures from g-Expectations}

We now look at the connection between g-expectations, introduced by Peng [1997] as a nonlinear expectations, and risk measures. Let the BSDE be given as;

$$
\begin{cases}d Y(t) & =g(t, Y(t), Z(t)) d t-Z(t) d B(t) . \\ Y(T) & =x\end{cases}
$$

then

Definition 12.36 (Conditional g-Expectation,Peng [1997] definition 36.1-36.5). The conditional g-expectation of $x$ under $\mathcal{F}_{t}$ for every $x \in L^{2}\left(\mathcal{F}_{T}\right)$ is defined as

$$
\mathcal{E}_{g}\left[-x \mid \mathcal{F}_{t}\right]:=Y_{t},
$$

where $Y_{t}$ is (the first component of) the solution to the BSDE (12.4) with terminal condition $x$. In particular, for $t=0$,

$$
\mathcal{E}_{g}[-x]:=Y_{0}
$$

is called a g-expectation.

Definition 12.37. Let $g: \Omega \times[0, T] \times \mathbb{R} \times \mathbb{R}^{d} \rightarrow \mathbb{R}$ and let $\rho: L^{2}\left(\mathcal{F}_{T}\right) \rightarrow L^{2}(\mathbb{R})$ be defined as

$$
\rho=\left(\rho_{t}\right)_{t \in[0, T]}, \rho_{t}(x):=\mathcal{E}_{g}\left[-x \mid \mathcal{F}_{t}\right], \forall x \in L^{2}\left(\mathcal{F}_{t}\right)
$$

Theorem 12.38. Using the above definition we get two implications to dynamic risk measures;

1. If the functional $g$ is convex in $(y, z) \in \mathbb{R} \times \mathbb{R}^{d}$, then $\left(\rho_{t}\right)_{t \in[0, T]}$ defined as in Definition 12.37 is a dynamic convex risk measure. Moreover $\left(\rho_{t}\right)_{t \in[0, T]}$ is time-consistent and satisfies the axioms positivity, translation-invariance and constancy.

2. If the functional $g$ is sub-linear in $(y, z) \in \mathbb{R} \times \mathbb{R}^{d}$, then $\left(\rho_{t}\right)_{t \in[0, T]}$ defined as in Definition 12.37 is a dynamic coherent risk measure which is time-consistent.

Another dynamic risk measure that is time-consistent is the dynamic entropic risk measure with threshold, see Nadal [2008].

With the arise of a solid theory for risk measures, how to allocate risk capital by selecting a proper risk measure has become an important issue for further research.

\subsection{G-Brownian motion}

Lets build further on the notion of g-expectation constructed above to construct G-expectations and G-Brownian motions. This construction is from Peng [2007] and Peng [2006]. Let $\mathcal{H}$ be a lattice of real functions defined on $\Omega$ containing 1 .

Definition 12.39. A nonlinear expectation $E$ is a functional $\mathcal{H} \mapsto \mathbb{R}$ satisfying the following properties

1. Monotonicity: If $X, Y \in \mathcal{H}$ and $X \geq Y$ then $E[X] \geq E[Y]$.

2. Preserving of constants: $E[c]=c$.

3. Sub-additivity (or self-dominated property): $E[X]-E[Y] \leq E[X-Y]$, for all $X, Y \in \mathcal{H}$. 
4. Positive homogeneity: $E[\lambda X]=\lambda E[X]$, for all $\lambda \geq 0$, and $X \in \mathcal{H}$.

5. Constant translatability: $E[X+c]=E[X]+c$.

We can now define a $G$-normal distribution.

Definition 12.40. A real valued random variable $X$ with the standard $G$-normal distribution is characterized by its $G$-expectation defined by

$$
E[\phi(X)]=P_{1}^{G}(\phi):=u(1,0), \phi \in \operatorname{lip}(\mathbb{R}) \mapsto \mathbb{R}
$$

where $u=u(t, x)$ is a bounded continuous function on $[0, \infty) \times \mathbb{R}$ which is the (unique) viscosity solution of the following nonlinear parabolic partial differential equation (PDE):

$$
\left\{\begin{array}{l}
\partial_{t} u-G\left(\partial_{x x}^{2} u\right)=0 \\
u(0, x)=\phi(x) .
\end{array}\right.
$$




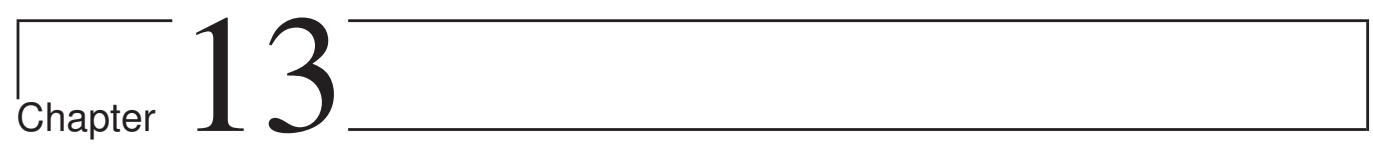

\section{Stochastic Differential Equations}

Consider the ordinary differential equation often used to model systems

$$
\begin{cases}\dot{X}(t)=f(X(t), t), & t>0 \\ X(0)=x_{0}, & x_{0} \in \mathbb{R} .\end{cases}
$$

If we want to model a system that contains noise it seems reasonable to add some noise to the above equation. A formal way to do so is to write

$$
\begin{cases}\dot{X}(t)=f(X(t), t)+g(X(t), t) \xi(t), & t>0, \\ X(0)=x_{0}, & x_{o} \in \mathbb{R} .\end{cases}
$$

where $\xi$ is "white noise"'". This approach presents us with these mathematical problems:

1. Define the "white noise" in a rigorous way.

2. Define what it means for $X$ to solve (13.1).

3. Show that (13.1) has a solution and investigate uniqueness.

To address (1) and (2) we use the Itô integral and define the stochastic differential equation by

$$
d X_{t}^{(i)}=b_{i}\left(X_{t}\right) d t+\sum_{j=1}^{r} \sigma_{i, j}\left(X_{t}\right) d W_{t}^{(j)}
$$

for an r-dimensional Brownian motion $W$. This equation should be interpreted as an informal way of expressing the corresponding Itô integral equation i.e.

$$
X_{t}^{(i)}=x_{0}+\int_{0}^{t} b_{i}\left(X_{s}\right) d s+\sum_{j=1}^{r} \int_{0}^{t} \sigma_{i, j}\left(X_{s}\right) d W_{s}^{(j)}
$$

Let

$$
a_{i, k}(t, x):=\sum_{j=1}^{r} \sigma_{i, j}(t, x) \sigma_{k, j}(t, x)
$$


be the diffusion matrix.

We now need to address the two remaining questions:

1. When do solution to the stochastic differential equation exist?

2. If there exist a solution is the solution unique?

We consider two kind of solutions to (13.2), namely strong and weak.

\subsection{Strong Solutions}

We first consider strong solution defined as:

Definition 13.1. A strong solution to (13.2) on a given probability space $(\Omega, \mathcal{F}, \mathbf{P})$ with a given Brownian motion $W$ and initial condition $\zeta$, is a process $X=\left\{X_{t}\right\}$ with continuous sample paths and such that

1. $X$ is adapted to the filtration $\left\{\mathcal{F}_{t}\right\}$;

2. $P\left[X_{0}=\zeta\right]=1$;

3. $P\left[\int_{0}^{t}\left\{\left|b_{i}\left(s, X_{s}\right)\right|+\sigma_{i, j}^{2}\left(s, X_{s}\right)\right\} d s<\infty\right]=1$;

4.

$$
X_{t}^{(i)}=x_{i}+\int_{0}^{t} b_{i}\left(X_{s}\right) d s+\sum_{j=1}^{r} \int_{0}^{t} \sigma_{i, j}\left(X_{s}\right) d W_{s}^{(j)} .
$$

We also have the notion of strong uniqueness.

Definition 13.2. Suppose when $X$ and $\tilde{X}$ is two strong solutions to (13.2) with initial condition $\zeta$ then $P\left[X_{t}=\tilde{X}_{t} ; 0 \leq t<\infty\right]=1$. We then say that strong uniqueness holds for the pair $(b, \sigma)$.

Under some Lipschitz conditions we can ensure a unique solution to (13.2).

Theorem 13.3. Suppose that the coefficients $b(t, x), \sigma(t, x)$ are locally Lipschitz in the space variable. Then strong uniqueness holds for (13.2).

\subsection{Weak Solutions}

Now we consider another type of solutions, namely weak solutions. As the name suggest it is a weaker notion than strong solutions. In weak solutions we let the probability space be a part of the solution. A simple method to create weak solutions is to use the Girsanov theorem. Let us start by giving the definition of a weak solution.

Definition 13.4. A weak solution of (13.2) is a triple $\left((X, W),(\Omega, \mathcal{F}, \mathbf{P}),\left\{\mathcal{F}_{t}\right\}\right)$, where

1. $(\Omega, \mathcal{F}, \mathbf{P})$ is a probability space, and $\left\{\mathcal{F}_{t}\right\}$ is a filtration of sub- $\sigma$-fields of $\mathcal{F}$ satisfying the usual conditions; 
2. $X=\left\{X_{t}, \mathcal{F}_{t}, 0 \leq t<\infty\right\}$ is a continuous, adapted $\mathbb{R}^{d}$-valued process;

3. $W=\left\{W_{t}, \mathcal{F}_{t}, 0 \leq t<\infty\right\}$ is an $r$-dimensional Brownian motion, and 4.

$$
X_{t}^{(i)}=x_{i}+\int_{0}^{t} b_{i}\left(X_{s}\right) d s+\sum_{j=1}^{r} \int_{0}^{t} \sigma_{i, j}\left(X_{s}\right) d W_{s}^{(j)}
$$

As before we have to define what we mean by unique, but this time we will have two different notions.

Definition 13.5. 1. If $\left((X, W),(\Omega, \mathcal{F}, \mathbf{P}),\left\{\mathcal{F}_{t}\right\}\right)$, and $\left((\tilde{X}, W),(\Omega, \mathcal{F}, \mathbf{P}),\left\{\tilde{\mathcal{F}}_{t}\right\}\right)$, are two solutions to (13.2) such that $P\left[X_{0}=\tilde{X}_{0}\right]=1$ and they are indistinguishable, that is $P\left[X_{t}=\tilde{X}_{t}\right.$, for all $\left.0 \leq t<\infty\right]=1$. The we say that pathwise uniqueness holds for $(13.2)$

2. We say that uniqueness in the sense of probability law holds for equation (13.2) if, for any two weak solutions $\left((X, W),(\Omega, \mathcal{F}, \mathbf{P}),\left\{\mathcal{F}_{t}\right\}\right)$, and $\left((\tilde{X}, \tilde{W}),(\tilde{\Omega}, \tilde{\mathcal{F}}, \tilde{\mathbf{P}}),\left\{\tilde{\mathcal{F}}_{t}\right\}\right)$ with the same initial distribution, i.e.,

$$
P\left[X_{0} \in \Gamma\right]=P\left[\tilde{X}_{0} \in \Gamma\right]
$$

for all $\Gamma \in \mathcal{B}(\mathbb{R})$, the two processes $X$ and $\tilde{X}$ have the same law.

For a solution $(X, W),(\Omega, \mathcal{F}, \nu)$ to (13.2) we let $Y_{t}=X_{t}-X_{0}$ and consider the triple $\left(X_{0}, W, Y\right)$ which induce a measure $P$ on

$$
(\Theta, \mathcal{B}(\Theta)):=\left(\mathbb{R}^{d} \times C[0, \infty)^{r} \times C[0, \infty)^{d}, \mathcal{B}\left(\mathbb{R}^{d}\right) \times \mathcal{B}\left(C[0, \infty)^{r}\right) \times \mathcal{B}\left(C[0, \infty)^{d}\right)\right)
$$

where

$$
P(A):=\nu\left[\left(X_{0}, W, Y\right) \in A\right]
$$

and

$$
\mu(B):=\nu\left[X_{o} \in B\right]
$$

for $B \in \mathcal{B}\left(\mathbb{R}^{d}\right)$ and $A \in \mathcal{B}(\Theta)$.

We have the following important result by Yamada and Watanabe.

Proposition 13.6. Pathwise uniqueness implies uniqueness in the sense of probability law.

Proof. Proposition 5.3.20 in Karatzas and Shreve [2000].

From this proposition we get the following important corollary.

Corollary 13.7. Suppose the stochastic differential equation (13.2) has a weak solution $(X, W)$, $(\Omega, \mathbf{P}, \mathcal{F}),\left\{\mathcal{F}_{t}\right\}$ with initial distribution $\mu$, and suppose that pathwise uniqueness holds. Then 
there exists a $\mathcal{B}(\mathbb{R}) \otimes \mathcal{B}\left(C[0, \infty)^{r} / \mathcal{B}\left(C[0, \infty)^{d}\right)\right.$-measurable function $h: \mathbb{R}^{d} \times C[0, \infty)^{r} \mapsto$ $C[0, \infty)^{d}$, which is also $\hat{\mathcal{B}} / \mathcal{B}_{t}(C[0, \infty))$-measurable for every fixed $0 \leq t<\infty$, such that

$$
X .=h\left(X_{0}, W .\right), \text { a.s. } \mathbf{P} \text {. }
$$

Here, $\hat{\mathcal{B}}$ is the augmentation of the filtration $\mathcal{B}(\mathbb{R}) \otimes \mathcal{B}\left(C[0, \infty)^{r}\right.$ by the null sets of $\mu(d x) \mathbf{P}_{*}(d \omega)$ and $\mathbf{P}_{*}$ is the marginal on the $\omega$-coordinate. Moreover, given any probability space $(\tilde{\Omega}, \tilde{\mathcal{F}}, \tilde{\mathbf{P}})$ rich enough to support an $\mathbb{R}^{d}$-valued random variable $\xi$ with distribution $\mu$ and an independent Brownian motion $\left\{\tilde{W}, \mathcal{F}^{\tilde{W}} ; 0 \leq t<\infty\right\}$, the processes

$$
\tilde{X} .:=h(\xi, \tilde{W} .) .
$$

is a strong solution of the equation (13.2) with initial condition $\xi$.

\subsection{Stochastic Differential Equations for Lévy Processes}

Consider the SDE

$$
\begin{aligned}
d Z_{t} & =b\left(Z\left(t^{-}\right)\right) d t+\sigma\left(Z\left(t^{-}\right) d W(t)+\int_{|x|<c} F\left(Z\left(t^{-}\right), x\right) \tilde{N}(d t, d x)\right. \\
& +\int_{|x| \geq c} G\left(Z\left(t^{-}\right), x\right) \tilde{N}(d t, d x)
\end{aligned}
$$

with initial condition $Z(0)=Z_{0}$. Assume that

1. Lipschitz Condition: There exists $K_{1}>0$ such that for all $y_{1}, y_{2} \in \mathbb{R}^{d}$ we have

$$
\begin{gathered}
\left|b\left(y_{1}\right)-b\left(y_{2}\right)\right|^{2}+\left\|a\left(y_{1}, y_{1}\right)-2 a\left(y_{1}, y_{2}\right)+a\left(y_{2}, y_{2}\right)\right\| \\
+\int_{|x|<c}\left|F\left(y_{1}, x\right)-F\left(y_{1}, x\right)\right|^{2} \nu(d x) \leq K_{1}\left|y_{1}-y_{2}\right|^{2} .
\end{gathered}
$$

2. Growth Condition: There exists $K_{2}>0$ such that for all $y \in \mathbb{R}^{d}$ we have

$$
|b(y)|^{2}+\|a(y, y)\|+\int_{|x|<c}|F(y, x)|^{2} \nu(d x) \leq K_{2}\left(1+|y|^{2}\right)
$$

3. The mapping $y \rightarrow G(y, x)$ is continuous.

Assume the above conditions then we have the following theorem.

Theorem 13.8. There exists a unique solution $Z=\{Z(t)\}$ to the equation (13.4) such that $Z(0)=Z_{0}$ (a.s) such that $Z_{0}$ if $\mathcal{F}_{0}$-measurable (standard initial condition). The process $Z$ is adapted and càdlàg.

Proof. See theorem 6.2.3 in Applebaum [2009]. 


\subsection{Stochastic Flows and Cocycles}

Let $Y_{y}$ be the strong solution to the equation (13.4) with fixed deterministic initial condition $Y_{0}=y$. Define $\Phi: \mathbb{R}^{d}: \times \Omega \rightarrow \mathbb{R}^{d}$ by

$$
\Phi_{s, t}(y, \omega)=y+Y_{y}(t)(\omega)-Y_{y}(s)(\omega) .
$$

Definition 13.9. We say $\Phi$ is a stochastic flow if there exists $\mathcal{N} \subset \Omega$ with $\mathbf{P}(\mathcal{N})=0$, such that for all $\omega \in \Omega \backslash \mathcal{N}$

1. $\Phi_{r, t}^{\omega}=\Phi_{s, t}^{\omega} \circ \Phi_{r, s}^{\omega}$;

2. $\Phi_{s, s}^{\omega}=y$;

If, in addition, each $\Phi_{s, t}^{\omega}$ is a homeomorphism of $\mathbb{R}^{d}$ for all $\omega \in \Omega \backslash \mathcal{N}$, we say that $\Phi$ is a stochastic flow of homeomorphisms. If in addition we have

1. the random variables $\left\{\Phi_{t_{j}, t_{j+1}} ; \leq j \leq n-1\right\}$ are independent;

2. the mappings $t \rightarrow \Phi_{s, t}(y)$ are càdlàg.

Then we say that $\Phi$ is a Lévy flow. If we can strengthen the càdlàg property to continuous then we say that $\Phi$ is a Brownian flow.

Consider the two parameter version of the equation (13.4):

$$
\begin{aligned}
d \Phi_{s, t}(y) & =b\left(\Phi_{s, t^{-}}(y)\right) d t+\sigma\left(\Phi_{s, t^{-}}(y)\right) d W(t)+\int_{|x|<c} F\left(\Phi_{s, t^{-}}(y), x\right) \tilde{N}(d t, d x) \\
& +\int_{|x| \geq c} G\left(\Phi_{s, t^{-}}(y), x\right) \tilde{N}(d t, d x) .
\end{aligned}
$$

Then

Theorem 13.10. $\Phi$ is a Lévy flow.

Proof. See theorem 6.4.2 in Applebaum [2009]. 


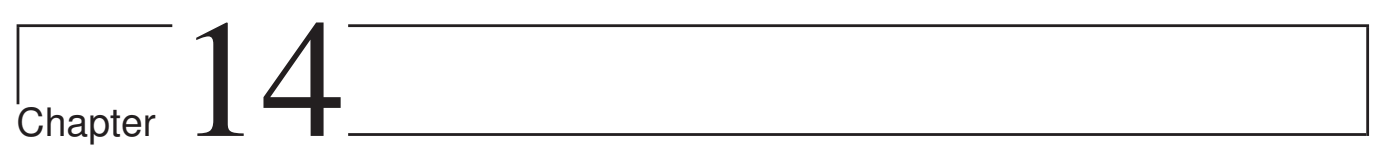

\section{Backward Stochastic Differential Equations}

To solve stochastic differential equation where the equation is given for the final time is not as easy as it is for deterministic systems. We need to ensure that the solution is adapted. So to solve this there have been developed an extensive theory. We will present small parts of it.

This chapter is based on Zhang [2009], Ma and Yong [2007],Øksendal and Zhang [2001], Peng and Shi [2000], Gianin [2002a] and Haadem [2009].

\subsection{Background}

First, let

- $L_{T}^{2, d}\left(\mathbb{R}^{d}\right)$ : is the space of all $\mathcal{F}_{t^{-}}$measurable r.v. $X: \Omega \rightarrow \mathbb{R}^{d}$ s.t. $E\left[|X|^{2}\right]<\infty$.

- $H_{T}^{2}\left(L_{2}\right)$ : space of all predictable processes $\phi: \Omega \times[0, T] \rightarrow \mathbb{R}^{d}$ s.t

$$
\|\phi\|^{2}=E\left[\int_{0}^{T}\left|\phi_{s}\right|^{2} d s\right]<\infty .
$$

Next, for $\beta>0$ we define $\|\phi\|_{\beta}=E\left[\int_{0}^{T} e^{\beta t}\left|\phi_{s}\right|^{2} d s\right]$ so that;

- $H_{T, \beta}^{2}$ : is the space $H_{T}^{2}$ endowed with the norm $\|\cdot\|_{\beta}$. (Its easily seen that $\|\cdot\|_{\beta}$ and $\|\cdot\|$ are equivalent.)

- $L^{2} \mathcal{F}_{T}\left(\Omega, W^{1, \infty}\left(\mathbb{R}^{n} ; \mathbb{R}^{m}\right)\right)$ is the set of all functions $f:[0, T] \times M \times \Omega \rightarrow N$, such that for any fixed $\theta \in M,(t, \omega) \rightarrow f(t, \omega)$ is $\left\{\mathcal{F}_{t}\right\}_{t \geq 0^{-}}$progressively measurable with $f(t, 0, \omega) \in$ $L_{\mathcal{F}}^{2}([0, T] ; N)$, and there exists a constant $\bar{L}>$, such that

$$
|f(t, \theta, \omega)-f(t, \bar{\theta}, \omega)| \leq L|\theta-\bar{\theta}|, \forall \theta, \bar{\theta} \in M \text {, a.e. } t \in[0, T] \text {, a.s.; }
$$

We are now ready to give the definition of BSDEs;

Definition 14.1. A backward stochastic differential equations (BSDE) is equation on the form

$$
\left\{\begin{array}{l}
-d Y(t)=g(t, Y(t), Z(t)) d t-Z(t) d B(t) . \\
Y(T)=\xi
\end{array}\right.
$$


and where $(Y, Z)$ is a solution to the BSDE such that $Y(t), t \in[0, T]$ is a continuous adapted process and $Z(t), t \in[0, T]$ is a predictable process satisfying $\int_{0}^{T}|Z(t)|^{2} d s<\infty P$-a.s. and

$$
Y(t)=\xi+\int_{t}^{T} g(s, Y(s), Z(s)) d s-\int_{t}^{T} Z(s) d B(s), P-a . s .
$$

Definition 14.2. We say that $(f, \xi)$ is a pair of standard parameters for the BSDE if they satisfy

- $\xi \in L_{T}^{2}$.

- $f(\cdot, 0,0) \in H_{T}^{2}$.

- $f$ is uniformly Lipschitz.

\subsection{Solutions of BSDEs}

Under different conditions we get some existence and uniqueness results.

Theorem 14.3 (Existence and uniqueness of solution, Zhang [2009] and also in Ma and Yong [2007] section 4). Let $(f, \xi)$ be a pair of standard parameters for the BSDE (14.2), then there exists an unique pair $(Y, Z) \in H_{T}^{2} \times H_{T}^{2}$ which solves the BSDE (14.2).

Let $V, H$ be two separable Hilbert spaces such that $C$ is continuously, densely imbedded in $H$.Let $A$ be a bounded linear operator from $V$ to $V^{*}$ such that there exists constants $\alpha>0$ and $\lambda \geq 0$ such that

$$
2\langle A u, u\rangle+\lambda|u|_{h}^{2} \geq \alpha\|u\|_{V}^{2}
$$

for all $u \in V$. Let $B_{t}$ be a cylindrical Brownian motion with covariance space $K$ (see chapter 23). Let us further require that there exists a constant $c<\infty$ such that

$$
\left|b\left(t, y_{1}, z_{1}\right)-b\left(t, y_{2}, z_{2}\right)\right|_{H} \leq c\left(\left|y_{1}-y_{2}\right|_{H}+\left|z_{1}-z_{2}\right|_{L_{2}(K, H)}\right)
$$

for all $(t, y, z) \in[0, T] \times H \times L_{2}(K, H)$. Further assume

$$
E\left[\int_{0}^{T} \mid b(t, 0,0)_{H}^{2} d t\right]<\infty .
$$

Consider now the BSDE

$$
\begin{aligned}
d Y(t, x) & =A Y(t, x) d t+b(t, x, Y(t, x), Z(t, x)) d t+Z(t, x) d B(t),(t, x) \in(0, T) \times \mathbb{R}^{n} \\
Y(T, x) & =\phi(x) .
\end{aligned}
$$

Theorem 14.4 (Theorem 3.1 in Øksendal and Zhang [2001]). Assume $E\left[|\phi|_{H}^{2}\right]<\infty$. Then there exists an unique $H \times L_{2}(K, H)$-valued progressively measurable process $\left(Y_{t}, Z_{t}\right)$ such that

(i) $E\left[\int_{0}^{T}\left|Y_{t}\right|_{H}^{2}\right]<\infty$ and $E\left[\int_{0}^{T}\left|Z_{t}\right|_{H}^{2}\right]<\infty$

(ii) $\phi=Y_{t}+\int_{t}^{T} A Y_{s} d s+\int_{t}^{T} b\left(s, Y_{S}, Z_{S}\right) d s+\int_{t}^{T} Z_{s} d B_{s} ; 0 \leq t \leq T$. 


\subsection{BSDE with Concave Generator}

Assume the generator $f(t, y, z)$ is concave w.r.t. $(y, z)$. Define

$$
F(t, \beta, \gamma)=\sup _{(y, z) \in \mathbb{R} \times \mathbb{R}^{n}}[f(t, y, z)-\beta y-\gamma z] .
$$

Since $f$ is concave and continuous, we have from concave analysis that

$$
f(t, y, z)=\sup _{(\beta, \gamma) \in D_{F}}\{F(t, \beta, \gamma)+\beta y+\gamma z\}
$$

where we let, for a pair of predictable processes

$$
f^{\beta, \gamma}(t, y, z):=F(t, \beta, \gamma)+\beta y+\gamma z \text { which is linear in }(y, z)
$$

and

$$
D_{F}=\{(\beta, \gamma): F(t, \beta, \gamma)<\infty\} \subset[-C, C]^{n+1}
$$

Let

$$
A=\left\{\left(\beta_{t}, \gamma_{t}\right): E\left[\int_{0}^{T} F\left(t, \beta_{t}, \gamma_{t}\right)^{2} d t\right]<\infty\right\},
$$

be the set of admissible controls. Then we have

Theorem 14.5. There exists an optimal control $(\bar{\beta}, \bar{\gamma}) \in A$ s.t.

$$
f\left(t, Y_{t}, Z_{t}\right)=f^{\bar{\beta}, \bar{\gamma}}\left(t, Y_{t}, Z_{t}\right) .
$$

Proposition 14.1. Let $f$ be a concave standard parameter and define

$$
f^{\beta, \gamma}(t, y, z)=F\left(t, \beta_{t}, \gamma_{t}\right)+\beta_{t} y+\gamma_{t} z
$$

Then for any $t \geq 0$,

$$
Y_{t}=\underset{(\beta, \gamma) \in A}{\operatorname{essinf}}\left\{Y^{\beta, \gamma}(t)\right\}
$$

where $Y^{\beta, \gamma}(t)$ is the solution of the BSDE associated with the linear generator $f^{\beta, \gamma}$

\subsection{Forward-Backward Stochastic Differential Equations}

We define a Forward-Backward stochastic differential equation (FBSDE) as follows:

$$
\left\{\begin{aligned}
d X(t) & =b\left(X(t), u_{0}(t), u_{1}(t)\right) d t+\sigma\left(X(t), u_{0}(t), u_{1}(t)\right) d B(t) \\
& +\int_{\mathbb{R}^{k}} \gamma\left(X\left(t^{-}\right), u_{2}(t, z), z\right) \tilde{N}(d t, d z) \\
d Y(t) & =-g(t, Y(t), Z(t), K(t)) d t+Z(t) d B(t)+\int_{\mathbb{R}^{k}} K(t, z) \tilde{N}(d t, d z) \\
X(0) & =x_{0}, Y\left(\tau_{S}\right)=-X\left(\tau_{S}\right)
\end{aligned}\right.
$$

Then we have that; 
Theorem 14.6 (Ma and Yong [2007] Theorem 5.1). Let $M=\mathbb{R}^{n} \times \mathbb{R}^{m} \times \mathbb{R}^{l}$, and b, $\sigma, h$ and g satisfy

$$
\left\{\begin{array}{l}
b \in L_{\mathcal{F}}^{2}\left([0, T], W^{1, \infty}\left(M ; \mathbb{R}^{n}\right)\right) \\
g \in L^{2} \mathcal{F}\left([0, T], W^{1, \infty}\left(M ; \mathbb{R}^{m}\right)\right) \\
h \in L^{2} \mathcal{F}_{T}\left(\Omega, W^{1, \infty}\left(\mathbb{R}^{n} ; \mathbb{R}^{m}\right)\right) \\
\sigma \in L^{2} \mathcal{F}\left([0, T], W^{1, \infty}\left(M ; \mathbb{R}^{n \times d}\right)\right)
\end{array}\right.
$$

Moreover, we assume that

$$
\left\{\begin{array}{l}
|\sigma(t ; x ; y ; z ; \omega)-\sigma(t ; x ; y ; z ; \omega)| \leq L_{0}|z-\bar{z}| \\
\forall(x, y) \in \mathbb{R}^{n} \times \mathbb{R}^{n} ; z ; \bar{z} \in \mathbb{R}^{m \times d} ; \text { a.e. } t>0 ; \text { a.s. } \\
\mid g(x ; \omega)-g x ; \omega)\left|\leq L_{1}\right| x-\bar{x} \mid ; \forall x ; \bar{x} \in \mathbb{R}^{n} \text { a.s. }
\end{array}\right.
$$

with

$$
L_{0}, L_{1}<1 \text {. }
$$

Then there exists a $T_{0}>0$, such that for any $T \in\left(0, T_{0}\right]$ and any $x \in \mathbb{R}^{d}$ (14.5) admits an unique adapted solution $(X ; Y ; Z) \in M$.

\subsubsection{Backward Stochastic Differential Equations and g-Expectations}

From the above Peng and Shi [2000] and Gianin [2002a] defined the g-expectation and the conditional g-expectation as follows:

Definition 14.7. For every $x \in L_{T}^{2}\left(\mathcal{F}_{t}\right)$ and for every $t \in[0, T]$ the conditional g-expectation of $x$ under $\mathcal{F}_{t}$ is defined by

$$
\mathcal{E}_{g}\left[x \mid \mathcal{F}_{t}\right]:=Y(t),
$$

where $Y(t)$ is the first component of the solution to the FBSDE (14.2) with terminal condition $\xi=x$. For $t=0$ we have

$$
\mathcal{E}_{g}[x]:=Y(0),
$$

which is called the g-expectation. 


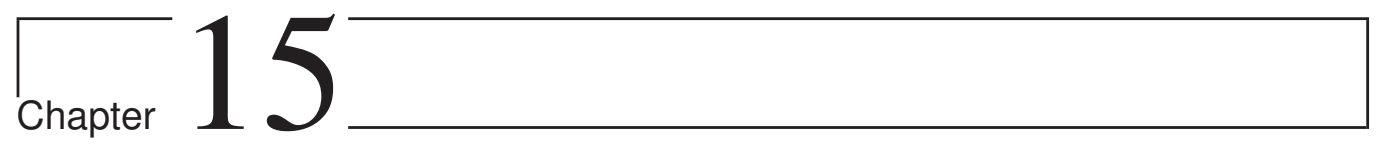

\section{Dirichlet Forms}

A deep and far reaching field of mathematics is the theory of Dirichlet forms. A Dirichlet form is a Markovian closed symmetric form on a $L^{2}$-space. The theory of Dirichlet forms originated in the work of Beurling and Deny $(1958,1959)$ on Dirichlet spaces.

This chapter is based on Fukushima et al. [1994], Rudin [1991] and Applebaum [2009].

\subsection{Unbound Operators in Banach Spaces}

Let $B$ be a real Banach space. Let $T_{1}$ and $T_{2}$ be linear operators in $\mathrm{B}$ with domains $D_{T_{1}}$ and $D_{T_{2}}$ respectively. We say that $T_{2}$ is an extension of $T_{1}$ if

1. $D_{T_{1}} \subseteq D_{T_{2}}$,

2. $T_{1} \psi=T_{2} \psi$ for all $\psi \in D_{T_{1}}$.

Denote this by $T_{1} \subseteq T_{2}$.

Proposition 15.1. A densely defined bounded linear operator $T$ in $B$ has a unique bounded extension whose domain is the whole of $B$,

Proof. See Applebaum [2009] Proposition 3.8.1.

As unbounded operators are not continuous we need a weaker concept. Start with defining the graph, $G_{T}$, of an operator $T$ as

$$
G_{T}:=\left\{(\phi, T \phi) ; \phi \in D_{T}\right\}
$$

We say that $T$ is closed if $G_{T}$ is closed in $B \times B$. Often a linear operator may only not be closed because its domain is too small. So we say that a linear operator $T$ in $B$ is closable if it has a closed extension $\tilde{T}$. The set $\rho(T)=\{\lambda \in \mathbb{C} ; \lambda I-T$ is invertible $\}$ is called the resolvent set to the operator $T$. The spectrum is defined as $\sigma(T):=\rho(T)^{c}$.

A linear operator $T$ is called self-adjoint if $T=T^{*}$ and essentially self-adjoint if it has a unique self-adjoint extension. Any self-adjoint operator can be built in a canonical way from projections. An important theorem is the spectral theorem. It is the starting point for a vast research area of functional analysis called operator theory. 
Theorem 15.2 (Spectral Theorem). Let A be a bounded self-adjoint operator on a Hilbert space $H$. Then there is a measure space $(X, \Omega, \mu)$ and a real-valued essentially bounded measurable function $f$ on $X$ and a unitary operator $U: H \mapsto L_{2}(\mu(X))$ such that

$$
U^{*} T U=A
$$

where $T$ is the multiplication operator:

$$
[T \varphi](x)=f(x) \varphi(x)
$$

and

$$
\|T\|=\|f\|_{\infty} .
$$

\subsubsection{Closed symmetric forms}

Suppose $D$ is a dense linear subspace of a real Hilbert space $B$. A closed symmetric form in $B$ is a bilinear map $\mathcal{E}: D \times D \rightarrow \mathbb{R}$ such that;

1. $\mathcal{E}$ is positive, i.e. $\mathcal{E}(f, f) \geq 0$ for all $f \in D$;

2. $\mathcal{E}$ is symmetric, i.e. $\mathcal{E}(f, g)=\mathcal{E}(g, f)$ for all $f, g \in D$;

3. $D$ is a real Hilbert space with respect to the inner product $\langle\cdot, \cdot\rangle_{\mathcal{E}}$, where

$$
\langle f, g\rangle_{\mathcal{E}}:=\langle f, g\rangle+\mathcal{E}(f, g)
$$

for each $f, g \in D$.

Theorem 15.3. There is a one-to-one correspondence between closed symmetric forms in $B$ and positive self-adjoint operators in $B$.

\subsubsection{Fourier Transform and Pseudo-Differential Operator}

For a integrable function $f: \mathbb{R} \rightarrow \mathbb{C}$ we define the Fourier transform as

$$
\hat{f}(u)=\frac{1}{(2 \pi)^{\frac{d}{2}}} \int_{\mathbb{R}^{d}} e^{-i(u, x)} f(x) d x
$$

for $u \in \mathbb{R}^{d}$.

Theorem 15.4 (Riemann-Lebesgue). If $f \in L^{2}\left(\mathbb{R}^{d}, \mathbb{C}\right)$ then $\hat{f} \in C_{0}\left(\mathbb{R}^{d}, \mathbb{C}\right)$ and $\|\hat{f}\|_{0} \leq\|f\|_{1}$.

We have that the Fourier transform is an isometry $L^{2}\left(\mathbb{R}^{d}, \mathbb{C}\right) \rightarrow L^{2}\left(\mathbb{R}^{d}, \mathbb{C}\right)$.

Theorem 15.5 (Plancherel). If $f \in L^{2}\left(\mathbb{R}^{d}, \mathbb{C}\right)$ then

$$
\int_{\mathbb{R}^{d}}|f(x)|^{2} d x=\int_{\mathbb{R}^{d}}|\hat{f}(u)|^{2} d u .
$$

Also, 
Theorem 15.6 (Parseval). If $f, g \in L^{2}\left(\mathbb{R}^{d}, \mathbb{C}\right)$ then

$$
\int_{\mathbb{R}^{d}} \overline{f(x)} g(x) d x=\int_{\mathbb{R}^{d}} \overline{\hat{f}(u)} \hat{g}(u) d u .
$$

Let $\alpha=\left(\alpha_{1}, \ldots, \alpha_{n}\right)$ be a multi-index so that $\alpha \in(\mathbb{N} \cap\{0\})^{d}$. Then let

$$
D^{\alpha}=\frac{1}{i^{|\alpha|}} \frac{\partial^{\alpha_{1}}}{\partial x_{1}^{\alpha_{1}}} \cdots \frac{\partial^{\alpha_{n}}}{\partial x_{n}^{\alpha_{n}}} .
$$

Define the $S$ chwartz space, $\mathcal{S}\left(\mathbb{R}^{d}, \mathbb{C}\right)$ to be the linear space of all $f \in C^{\infty}\left(\mathbb{R}^{d}, \mathbb{C}\right)$ such that

$$
\sup _{x \in \mathbb{R}^{d}}\left|x^{\beta} D^{\alpha} f(x)\right|<\infty,
$$

for all $\alpha, \beta$. The space $\mathcal{S}\left(\mathbb{R}^{d}, \mathbb{C}\right)$ is a Fréchet space with respect to the family of norms $\left\{\|\cdot\|_{N}\right\}_{N \in \mathbb{N} \cup\{0\}}$ where

$$
\|f\|_{N}:=\max _{|\alpha| \leq N} \sup _{x \in \mathbb{R}^{d}}\left(1+|x|^{2}\right)^{N}\left|D^{\alpha} f(x)\right|
$$

for each $f \in \mathcal{S}\left(\mathbb{R}^{d}, \mathbb{C}\right)$.

\subsection{Closed Forms and Semigroups}

Let us consider a family $\left\{T_{t}\right\}_{t \geq 0}$ of linear operators on a Hilbert space $H$ satisfying the following:

1 each $T_{t}$ is a symmetric operator with domain $\mathcal{D}\left(T_{t}\right)=H$;

2 semigroup property: $T_{t} T_{s}=T_{t+s}$, for all $t, s>0$;

3 contraction property: $\left(T_{t} u, T_{t} u\right) \leq(u, u)$ for all $t>0, u \in H$;

$4\left(T_{t} u-u, T_{t} u-u\right) \rightarrow 0$, for all $t \downarrow 0, u \in H$.

A family satisfying (1)-(3) is called a semigroup. If it satisfies (4) in addition it is called strongly continuous. Also, consider a another family $\left\{G_{\alpha}\right\}_{\alpha \geq 0}$ of linear operators on $H$

1 each $G_{\alpha}$ is a symmetric operator with domain $\mathcal{D}\left(G_{\alpha}\right)=H$;

2 resolvent equation: $G_{\alpha}-G_{\beta}+(\alpha-\beta) G_{\alpha} G_{\beta}=0$;

3 contraction property: $\left(\alpha G_{\alpha} u, \alpha G_{\alpha} u\right) \leq(u, u)$ for all $\alpha>0, u \in H$;

$4\left(\alpha G_{\alpha} u-u, \alpha G_{\alpha} u-u\right) \rightarrow 0$, as $\alpha \rightarrow \infty, u \in H$.

A family satisfying (1)-(3) is called a resolvent. If it satisfies (4) in addition it is called strongly continuous. 


\subsubsection{Connections}

Given a strongly continuous semigroup, $\left\{T_{t}\right\}_{t>0}$, on $H$ we have that

$$
G_{\alpha} u=\int_{0}^{\infty} e^{-\alpha t} T_{t} u d t
$$

define a strongly continuous resolvent, $\left\{G_{\alpha}\right\}_{\alpha>0}$, on $\mathrm{H}$. This is the resolvent of a given semigroup. The generator, $A$, of a strongly continuous semigroup, $\left\{T_{t}\right\}_{t>0}$, on $H$ is defined by

$$
\left\{\begin{array}{l}
A u=\lim _{t \rightarrow 0} \frac{T_{t} u-u}{t}, \\
D(A)=\{u \in H ; A u \text { exists as a strong limit }\} .
\end{array}\right.
$$

We can see that $G_{\alpha}$ is invertible such that

$$
\begin{cases}A u & =\alpha u-G_{\alpha}^{-1} u \\ D(A) & =G_{\alpha}(H)\end{cases}
$$

This operator, $A$ is called the generator of the resolvent $\left\{G_{\alpha}\right\}_{\alpha>0}$.

\section{Lemma 15.7.}

1. The generator of a strongly continuous resolvent is a non-positive definite self-adjoint operator.

2. The generator of a strongly continuous semigroup on $H$ coincides with the generator of its resolvent.

A symmetric operator $S$ on $H$ satisfying $D(S)=H, S^{2}=S$ is called a projection operator. A family $\left\{E_{\lambda}\right\}_{-\infty<\lambda<\infty}$ of projection operators is called a spectral family if

1. $\sup \left\{\left\|E_{\lambda}\right\| \mid \lambda \in \mathbb{R}\right\}<\infty$,

2. $E_{\lambda} E_{\mu}=\lambda, \lambda \leq \mu$,

3. $\lim _{\lambda^{\prime} \rightarrow \lambda} E_{\lambda^{\prime}} u=E_{\lambda} u$,

4. $\lim _{\lambda \rightarrow-\infty} E_{\lambda} u=0$,

5. $\lim _{\lambda \rightarrow \infty} E_{\lambda} u=u$,

for all $u \in H$. If the above holds then $0 \leq\left(E_{\lambda} u, u\right) \rightarrow(u, u), \lambda \rightarrow \infty$ for $u \in H$. Moreover, $\left(E_{\lambda} u, v\right)$ is of bounded variation in $\lambda$ for $u, v \in H$. Given a spectral family, $\left\{E_{\lambda}\right\}_{-\infty<\lambda<\infty}$, on $H$ an a continuous function $\phi(\lambda)$ on $\mathbb{R}$, a self-adjoint operator $A$ can be uniquely defined by

$$
\left\{\begin{array}{l}
(A u, v)=\int_{-\infty}^{\infty} \phi(\lambda) d\left(E_{\lambda} u, v\right), \text { for all } v \in H \\
D(A)=\left\{u \in H ; \int_{-\infty}^{\infty} \phi^{2}(\lambda) d\left(E_{\lambda} u, v\right)<\infty\right\}
\end{array}\right.
$$

This $A$ is denoted by $\int_{-\infty}^{\infty} \phi(\lambda) d E_{\lambda}$. Given a self-adjoint operator $A$ there exists a unique spectral family, $\left\{E_{\lambda}\right\}_{-\infty<\lambda<\infty}$, such that $A=\int_{-\infty}^{\infty} \lambda d E_{\lambda}$. This is called the spectral representation of $A$.

Lemma 15.8. Let $-A$ be a non-negative self-adjoint operator on $H$. Then 
1. $\left\{T_{t}=e^{t A}\right\}_{t>0}$ and $\left\{G_{\alpha}=(\alpha-A)^{-1}\right\}_{\alpha>0}$ are a strongly continuous semigroup and a strongly continuous resolvent on $H$ respectively.

2. The generator of $T_{t}$ coincides with $A$. the strongly continuous semigroup possessing $A$ as its generator is unique. The same goes for the resolvent.

We have a similar theorem by Bouleau and Hirsch [1991].

Theorem 15.9. The Following is equivalent:

1. $\left\{T_{t}\right\}_{t \geq 0}$ is a self-adjoint sub-Markovian semigroup in $L^{2}\left(\mathbb{R}^{d}\right)$ with infinitesimal generator A;

2. $A$ is a Dirichlet operator and $-A$ is poseive self-adjoint;

3. $\mathcal{E}(f)=\left\|(-A)^{\frac{1}{2}} f\right\|^{2}$ is a Dirichlet form with domain $D=D(\sqrt{(-A)})$.

Proof. See Bouleau and Hirsch [1991], pp.12-13.

Observe that

$$
\left\{\begin{array}{l}
D(\mathcal{E})=\left\{u \in H: \int[0, \infty) \lambda d\left(E_{\lambda} u, u\right)<\infty\right\} \\
\mathcal{E}(u, v)=\int[0, \infty) \lambda d\left(E_{\lambda} u, v\right)
\end{array}\right.
$$

where $\left\{E_{\lambda}\right\}$ is the spectral family associated with $-A$. Let us state the following theorem:

Theorem 15.10. There is a one to one correspondence between the family of closed symmetric forms $\mathcal{E}$ on $H$ and the family of non-positive definite, self-adjoint operators $A$ on $H$. The correspondence is given by:

$$
\left\{\begin{array}{l}
D(\mathcal{E})=D(\sqrt{-A}) \\
\mathcal{E}(u, v)=(\sqrt{-A} u, \sqrt{-A} v) .
\end{array}\right.
$$

Proof. See theorem 1.3.2 in Fukushima et al. [1994]. 


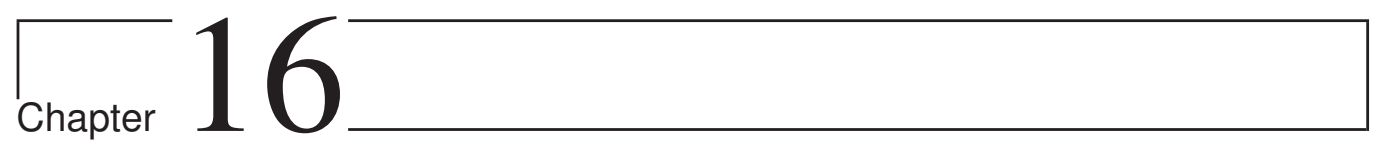

\section{Delay Equations}

As mentioned before many dynamic systems exhibits some kind of delay. It is therefore important to understand how the addition of a delay affects the system.

The chapter is based on Mohammed [2003] and lectures given by Salah-Eldin A. Mohammed Mohammed [2011].

\subsection{Notation}

We start by looking at a segment $x_{t}$ of a path $x$ over the the interval $[t, t+r]$, defines as a process on $J:=[-r, 0]$ by

$$
x_{t}(s):=x(t+s) \text { a.s., } s \in J .
$$

We now consider a SFDE

$$
\begin{cases}d x(t) & =h\left(t, x_{t}\right) d t+g\left(t, x_{t}\right) d W(t), t>0 \\ x_{0} & =\theta \in C\left([-r, 0], \mathbb{R}^{d}\right)\end{cases}
$$

on a filtered probability space $\left(\Omega, \mathcal{F},\{\mathcal{F}\}_{t \geq 0}, \mathcal{P}\right)$ satisfying the usual conditions and $W$ is a $m$ dimensional Brownian motion.. Denote by $C:=C\left([-r, 0], \mathbb{R}^{d}\right)$ equipped with the sup norm. For measurable functions from $\Omega \rightarrow C$ which is $L^{2}$ in the Bochner sense, see Chapter 23 for more details on Banach valued processes. We give $L^{2}(\Omega, C)$ the Banach norm

$$
\|\Phi\|_{L^{2}(\Omega, C)}:=\left[\int_{\Omega}\|\Phi(\omega)\|_{C}^{2} d P(\omega)\right]^{\frac{1}{2}} .
$$

In the above $h:[0, T] \times L^{2}(\Omega, C) \rightarrow L^{2}\left(\Omega, \mathbb{R}^{d}\right)$ is the drift coefficient and $g:[0, T] \times L^{2}(\Omega, C) \rightarrow$ $L^{2}\left(\Omega, \mathbb{R}^{d \times m}\right)$ is the diffusion coefficient satisfying:

1. The coefficient functionals $h$ and $g$ are jointly continuous and uniformly Lipschitz in the second variable with respect to the first, i.e.

$$
\left\|h\left(t, \phi_{1}\right)-h\left(t, \phi_{2}\right)\right\|_{L^{2}\left(\Omega, \mathbb{R}^{d}\right)}+\left\|g\left(t, \phi_{1}\right)-g\left(t, \phi_{2}\right)\right\|_{L^{2}\left(\Omega, \mathbb{R}^{d}\right)} \leq L\left\|\phi_{1}-\phi_{2}\right\|_{L^{2}(\Omega, C)}
$$


for all $t \in[0, T]$ and $\phi_{1}, \phi_{2} \in L^{2}(\Omega, C)$.

2. For each $\left\{\mathcal{F}_{t}\right\}$-adapted process $y:[0, T] \rightarrow L^{2}(\Omega, C)$, the process $h(\cdot, y(\cdot))$ and $g(\cdot, y(\cdot))$ are $\left\{\mathcal{F}_{t}\right\}$-adapted.

\subsection{Existence and Uniqueness}

We are now ready to give an existence and uniqueness result.

Theorem 16.1 (Existence and Uniqueness, Theorem I.1 in Mohammed [1998]). Suppose $h$ and $g$ satisfies the above requirements and let $\theta \in L^{2}\left(\Omega, C ; \mathcal{F}_{0}\right)$. Then the SFDE (16.1) has a unique solution ${ }^{\theta} x:[-r, \infty) \times \Omega \rightarrow \mathbb{R}^{d}$ starting of at $\theta \in L^{2}\left(\Omega, C ; \mathcal{F}_{0}\right)$ with $[0, T] \ni t \mapsto{ }^{\theta} x_{t} \in C$ sample-continuous, and ${ }^{\theta} x \in L^{2}\left(\Omega, C\left([-r, T], \mathbb{R}^{d}\right)\right)$ for all $T>0$. For a given $\theta$, uniqueness holds up to equivalence among all $\{\mathcal{F}\}_{0 \leq t \leq T}$-adapted processes in $L^{2}\left(\Omega, C\left([-r, T], \mathbb{R}^{d}\right)\right)$.

Proof. See Mohammed [1998]

Suppose now that $h$ and $g$ factors through deterministic functionals

$$
\begin{aligned}
& H:[0, T] \times C \rightarrow \mathbb{R}^{d}, \\
& G:[0, T] \times C \rightarrow \mathbb{R}^{d \times m} .
\end{aligned}
$$

Consider then the SFDE

$$
\begin{cases}d x(t) & =H\left(t, x_{t}\right) d t+G\left(t, x_{t}\right) d W(t), t>0 \\ x_{0} & =\theta \in C\left([-r, 0], \mathbb{R}^{d}\right)\end{cases}
$$

where $W$ is a $m$-dimensional Brownian motion. Assume further that we have the following.

1. Suppose that $H$ and $G$ are Lipschitz on bounded sets in $C$ uniformly in the second variable, i.e. for each $n \geq 1$ there exists a constant $L_{n}>0$ such that

$$
\left|H\left(t, \eta_{1}\right)-h\left(t, \eta_{2}\right)\right|+\left\|G\left(t, \eta_{1}\right)-g\left(t, \eta_{2}\right)\right\| \leq L_{n}\left\|\eta_{1}-\eta_{2}\right\|_{C}
$$

for all $t \in[0, T]$ and $\eta_{1}, \eta_{2} \in C$ with $\left\|\eta_{1}\right\|_{C} \leq n$ and $\left\|\eta_{2}\right\|_{C} \leq n$.

2. There is a constant $K>0$ such that

$$
|H(t, \eta)|+\|G(t, \eta)\| \leq K\left(1+\|\eta\|_{C}\right)
$$

for all $t \in[0, T]$ and $\eta \in C$.

Theorem 16.2 (Existence and Uniqueness, Theorem I.2 in Mohammed [1998]). Suppose H and $G$ satisfies the above requirements and let $\theta \in L^{2}\left(\Omega, C ; \mathcal{F}_{0}\right)$. Then the SFDE (16.1) has a unique $\{\mathcal{F}\}_{0 \leq t \leq T}$-adapted solution ${ }^{\theta} x:[-r, T] \times \Omega \rightarrow \mathbb{R}^{d}$ starting of at $\theta \in L^{2}\left(\Omega, C ; \mathcal{F}_{0}\right)$ with $[0, T] \ni t \mapsto{ }^{\theta} x_{t} \in C$ sample-continuous, and ${ }^{\theta} x \in L^{2}\left(\Omega, C\left([-r, T], \mathbb{R}^{d}\right)\right)$ for all $T>0$. For a given $\theta$, uniqueness holds up to equivalence among all $\{\mathcal{F}\}_{0 \leq t \leq T}$-adapted processes in $L^{2}\left(\Omega, C\left([-r, T], \mathbb{R}^{d}\right)\right)$. Furthermore, if $\theta \in L^{2 k}\left(\Omega, C ; \mathcal{F}_{0}\right)$ for some positive integer $k$, then ${ }^{\theta} x_{t} \in L^{2 k}\left(\Omega, C ; \mathcal{F}_{t}\right)$ and

$$
E\left\|^{\theta} x_{t}\right\|_{C}^{2 k} \leq C_{k}\left[1+\|\phi\|_{L^{2 k}(\Omega, C)}^{2 k}\right]
$$


for all $t \in[0 ; T]$ and some positive constant $C_{k}$.

Proof. See Mohammed [1998]

\subsection{Markov Property}

\section{Hypotheses $16.3(\mathrm{M})$.}

1. For each $t \geq 0, \mathcal{F}_{t}$ is the completion of the $\sigma$-algebra $\sigma\{W(u)\}_{0 \leq u \leq t}$;

2. $H$ and $G$ are jointly continuous and globally Lipschitz in the second variable uniformly with respect to the first, i.e.;

$$
\left|H\left(t, \eta_{1}\right)-H\left(t, \eta_{2}\right)\right|+\left\|G\left(t, \eta_{1}\right)-G\left(t, \eta_{2}\right)\right\| \leq L\left\|\eta_{1}-\eta_{2}\right\|_{c}
$$

for all $t \in[0, T]$ and $\eta_{1}, \eta_{2} \in C$.

Theorem 16.4 (Markov Property). Suppose Hypotheses (M) hold. Then the trajectory field $\left\{{ }^{\eta} x_{t}: t \geq 0, \eta \in C\right\}$ is a $C$-valued Feller process with transition probabilities:

$$
p\left(t_{1}, \eta, t_{2}, B\right):=P\left({ }^{\eta} x_{t_{2}}^{t_{1}} \in B\right),
$$

for all $t_{1} \leq t_{2}, B \in \mathcal{B}(C), \eta \in C$. In other words:

$$
P\left(X_{t_{2}} \in B \mid \mathcal{F}_{t_{1}}\right)=p\left(t_{1}, x_{t_{1}}(\cdot), t_{2}, B\right)=P\left(X_{t_{2}} \in B \mid x_{t_{1}}\right)
$$

a.s. Furthermore, if $H$ and $G$ do not depend on $t$, then the trajectory is time-homogeneous:

$$
p\left(t_{1}, \eta, t_{2}, \cdot\right)=p\left(0, \eta, t_{2}-t_{1}, \cdot\right)
$$

for all $0 \leq t_{1} \leq t_{2}, \eta \in C$.

Proof. See Theorem II.1 in Mohammed [2003]. 


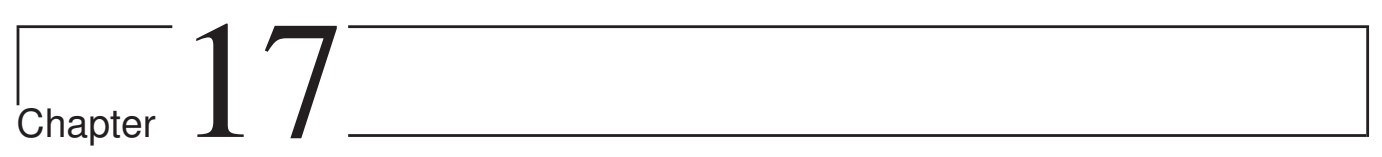

\section{Besov Spaces}

This chapter is about Banach spaces of functions related to fractional Sobolev spaces (or Sobolev-Slobodeckij spaces).

See Adams and Fournier [2003] for more information about these spaces.

Definition 17.1. Let $0<\alpha<2,1 \leq p<\infty$ and let $\Omega \subset \mathbb{R}^{d}$ be a Lipschitz domain. Then, the fractional Sobolev space $W^{\alpha, p}(\Omega)$ can be defined as

$$
W^{\alpha, p}(\Omega)=\left\{f: \Omega \longrightarrow \mathbb{R}:\|f\|_{W^{\alpha, p}(\Omega)}:=\left(\|f\|_{L^{p}(\Omega)}^{p}+[f]_{p, \alpha}^{p}\right)^{1 / p}<\infty\right\}
$$

Here,

$$
[f]_{p, \alpha}:=\left(\int_{\Omega} \int_{\Omega} \frac{|f(x)-f(y)|^{p}}{|x-y|^{d+2 \alpha}} d x d y\right)^{1 / p}
$$

denotes the Slobodeckij semi-norm.

The Sobolev-Slobodeckij spaces form a scale of Banach spaces, i.e. one has the continuous injections or embeddings

$$
W^{k+1, p}(\Omega) \hookrightarrow W^{s^{\prime}, p}(\Omega) \hookrightarrow W^{s, p}(\Omega) \hookrightarrow W^{k, p}(\Omega), \quad k \leq s \leq s^{\prime} \leq k+1 .
$$

Sobolev-Slobodeckij spaces are special cases of Besov spaces. See e.g. Adams and Fournier [2003].

Another approach to define fractional order Sobolev spaces $W^{\alpha, p}(\Omega)$ is

\section{Definition 17.2.}

$$
W^{\alpha, p}(\Omega):=\left\{f \in L^{p}(\Omega): \mathcal{F}^{-1}\left(1+|\xi|^{2}\right)^{\frac{\alpha}{2}} \mathcal{F} f \in L^{p}(\Omega)\right\}
$$

with the norm

$$
\|f\|_{W^{k, p}}:=\left\|\mathcal{F}^{-1}\left(1+|\xi|^{2}\right)^{\frac{k}{2}} \mathcal{F} f\right\|_{L^{p}},
$$

where $\mathcal{F}$ denotes the Fourier-transform. This space is also called a Bessel potential space. $\Omega$ is a domain with uniform $C^{k}$-boundary, $k$ a natural number and $1<p<\infty$. 
By the embeddings

$$
W^{k+1, p}\left(\mathbb{R}^{n}\right) \hookrightarrow W^{s^{\prime}, p}\left(\mathbb{R}^{n}\right) \hookrightarrow W^{s, p}\left(\mathbb{R}^{n}\right) \hookrightarrow W^{k, p}\left(\mathbb{R}^{n}\right), \quad k \leq s \leq s^{\prime} \leq k+1
$$

the Bessel potential spaces form a continuous scale between these Sobolev spaces. 


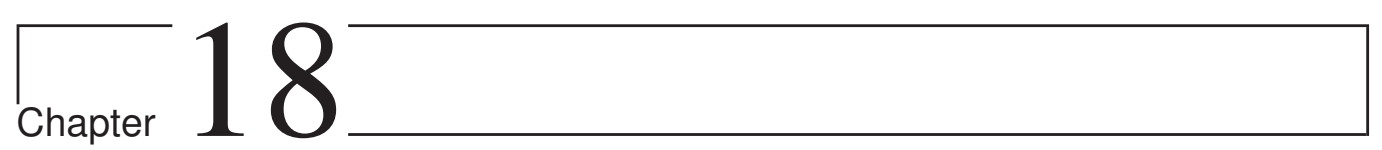

\section{Rough Paths}

Rough path analysis is the study of differential equations controlled by irregular paths such as a Wiener process. Rough path theory gives an approach to stochastic equations by viewing them as deterministic equations, for a fixed choice of driving path, which is in contrast to the more classical stochastic approach like the theory by Itô. The theory was developed in the 1990s by Terry Lyons.

This chapter is based on Lejay [2009],

\subsection{Hölder and Sobolev Spaces}

For $\beta \in(0,1)$ and $k, d \geq 1$, denote by $C_{b}^{\beta}\left(\mathbb{R}^{d}, \mathbb{R}^{k}\right)$ the space of bounded $\beta$-Hölder continuous functions, that is the space of continuous functions $u: \mathbb{R}^{d} \longrightarrow \mathbb{R}^{k}$ such that

$$
\|u\|_{C_{b}^{\beta}\left(\mathbb{R}^{d}, \mathbb{R}^{k}\right)}:=\|u\|_{\infty}+\sup _{x \neq y} \frac{|u(x)-u(y)|}{|x-y|^{\beta}}<\infty,
$$

where $\|u\|_{\infty}:=\sup _{x \in \mathbb{R}^{d}}|u(x)|$ and $|\cdot|$ is the Euclidean norm. We also simply write $C_{b}^{\beta}\left(\mathbb{R}^{d}\right)=$ $C_{b}^{\beta}\left(\mathbb{R}^{d}, \mathbb{R}\right)$. Further, we denote by $C_{b}^{i, \beta}\left(\mathbb{R}^{d}\right)$ for $i \geq 1$ and $0<\alpha<1$ the Banach space of all $i$-times Fréchet differentiable functions $u: \mathbb{R}^{d} \longrightarrow \mathbb{R}$ with $D^{l} u \in C_{b}^{\beta}\left(\mathbb{R}^{d},\left(\mathbb{R}^{d}\right)^{\otimes(l+1)}\right), l=1, \ldots, i$ and norm $\|\cdot\|_{C_{b}^{i, \beta}\left(\mathbb{R}^{d}\right)}$ given by

$$
\|u\|_{C_{b}^{i, \beta}\left(\mathbb{R}^{d}\right)}:=\|u\|_{\infty}+\sum_{l=1}^{i}\left\|D^{l} u\right\|_{\infty}+\sup _{x \neq y} \frac{|u(x)-u(y)|}{|x-y|^{\beta}}
$$

We let $C_{b}^{0, \beta}\left(\mathbb{R}^{d}\right):=C_{b}^{\beta}\left(\mathbb{R}^{d}\right)$. For notational convenience we also denote the norm of the Banach space $C\left([0, T], C_{b}^{i, \beta}\left(\mathbb{R}^{d}\right)\right)$ by $\|\cdot\|_{C_{b}^{i, \beta}}$ defined as

$$
\|u\|_{C_{b}^{i, \beta}}=\sup _{0 \leq t \leq T}\|u(t, \cdot)\|_{C_{b}^{i, \beta}\left(\mathbb{R}^{d}\right)} .
$$




\section{$18.2 p$-Variation and Hölder Continuous Paths}

Let $T>0$ and $\mathrm{x}$ a continuous path from $[0, T]$ to $\mathbb{R}^{d}$. Further, let $\Pi=\left\{t_{i}\right\}_{i=0, \ldots, k}$ be a partition of $[0, T]$ of $k$ elements. For $p \geq 1$, define

$$
\mathcal{P}(x, \Pi, p):=\sum_{i=0}^{k-1}\left|x_{t_{i+1}}-x_{t_{i}}\right|^{p} .
$$

We now define the $p$-variation of $x$ on $[s, t] \subset[0, T]$ by

$$
\operatorname{Var}_{p,[s, t]}(x)=\sup _{\text {all partitions } \Pi \text { of }[0, T]} \mathcal{P}\left(x_{\mid[s, t]}, \Pi \cup[s, t], p\right)^{\frac{1}{p}}
$$

Definition 18.1. A function $x:[0, T] \rightarrow \mathbb{R}^{d}$ is said to be of $p$-variation if $\operatorname{Var}_{p,[s, t]}(x)$ is finite.

Note that if $x$ is of finite $p$-variation it is also of finite $q$-variation for all $q>p$. This is seen simply by noting that

$$
\operatorname{Var}_{q,[0, T]}(x) \leq 2^{\frac{(q-p)}{q}}\|x\|_{\infty}^{\frac{(q-p)}{q}}\left(\operatorname{Var}_{p,[0, T]}(x)\right)^{\frac{p}{q}}
$$

A useful observation is that if $x$ is a path of finite $p$-variation and

$$
\phi(t)=\inf \left\{s>0: \operatorname{Var}_{p,[0, T]}(x)^{p}>t\right\},
$$

then $\phi$ is increasing and $x \circ \phi$ is $\frac{1}{p}$-Hölder continuous. Now, define the Hölder continuity modulus of a path $x:[0, T] \rightarrow \mathbb{R}^{d}, H_{\alpha}(x)$, by

$$
H_{\alpha}(x):=\sup _{0 \leq s \leq t \leq T} \frac{\left|x_{t}-x_{s}\right|}{|t-s|^{\alpha}} .
$$

As above with $p$-variation paths we note that any $\alpha$-Hölder continuous path $x$ is also $\beta$-Hölder continuous for all $\beta<\alpha$ with the analog inequality given by

$$
H_{\beta}(x) \leq 2^{1-\frac{\beta}{\alpha}}\|x\|_{\infty}^{1-\frac{\beta}{\alpha}}\left(H_{\alpha}(x)\right)^{\frac{\beta}{\alpha}}
$$

This is of course only a semi-norm so we denote, for $\alpha$-Hölder continuous paths $x:[0, T] \rightarrow \mathbb{R}^{d}$, $\|x\|_{\alpha}$ to be the norm defined by

$$
\|x\|_{\alpha}=\left|x_{0}\right|+H_{\alpha}(x)
$$

Further let $C^{\alpha}\left([0, T) ; \mathbb{R}^{d}\right)$ be the subset of functions $x$ in $C\left([0, T) ; \mathbb{R}^{d}\right)$ such that $\|x\|_{\alpha}$ is finite. From the Ascoli Theorem and (18.2) we get

Lemma 18.2. Let $\left\{x^{n}\right\}_{n \in \mathbb{N}}$ be such that $x^{n} \in C^{\alpha}\left([0, T) ; \mathbb{R}^{d}\right)$ and $\left\{\left\|x^{n}\right\|_{\alpha}\right\}_{n \in \mathbb{N}}$ is bounded. Then there exists $x$ in $C^{\alpha}\left([0, T) ; \mathbb{R}^{d}\right)$ and a subsequence $\left\{x^{n}\right\}_{n \in \mathbb{N}}$ that converges to $x$ with respect to $\|\cdot\|_{\beta}$ for all $\beta<\alpha$. 


\subsection{Integral Along $\alpha$-Hölder Continuous Paths, $\alpha \in\left(\frac{1}{2}, 1\right]$}

We let for simplicity $d=2$. The following theorem is due to Young [1936]

Theorem 18.3. Let $\alpha, \beta \in(0,1]$ with $\alpha+\beta>1$. Then

$$
(x, y) \mapsto\left(t \mapsto \int_{0}^{t} y_{s} d x_{s}\right)
$$

is bilinear and continuous from $C^{\alpha}([0, T) ; \mathbb{R}) \times C^{\beta}([0, T) ; \mathbb{R})$ to $C^{\alpha}([0, T) ; \mathbb{R})$.

Proof. See Theorem 1 in Lejay [2009].

Let $\mathcal{J}(x ; s, t)$ denote the Young integral

$$
\mathcal{J}(x ; s, t):=\int_{x_{\mid[s, t]}} f=\int_{s}^{t} f_{1}\left(x_{r}\right) d x_{r}^{1}+\int_{s}^{t} f_{2}\left(x_{r}\right) d x_{r}^{2}
$$

for a path $x$ on the interval $(S, T]$ where $0 \leq S \leq s \leq t \leq T$ and $y_{t}=f\left(x_{t}\right)$.

Corollary 18.4. Let $\alpha \in\left(\frac{1}{2}, 1\right]$ and $f \in \operatorname{Lip}\left(\gamma ; \mathbb{R}^{2} \rightarrow \mathbb{R}^{m}\right)$ with $\gamma>\frac{1}{\alpha}-1$. Then $\mathcal{J}(x ; s, t)$ is well defined as a Young integral on $C^{\alpha}\left([0, T] ; \mathbb{R}^{2}\right)$ and is a locally Lipschitz map from $\left(C^{\alpha}\left([0, T) ; \mathbb{R}^{2}\right),\|\cdot\|_{\alpha}\right)$ to $\left(C^{\alpha}\left([0, T) ; \mathbb{R}^{m}\right),\|\cdot\|_{\alpha}\right)$.

\subsection{Integral Along $\alpha$-Hölder Continuous Paths, $\alpha \in\left(\frac{1}{3}, \frac{1}{2}\right]$}

The problem when moving to $\alpha$ below $\frac{1}{2}$ is that we may have a sequence $\left\{x^{n}\right\}_{n \in \mathbb{N}} \subset C^{\alpha}\left([0, T) ; \mathbb{R}^{2}\right.$ and $\left\{z^{n}\right\}_{n \in \mathbb{N}} \subset C^{\alpha}\left([0, T) ; \mathbb{R}^{2}\right)$ both converging uniformly to a path $x$ in $C^{\alpha}\left([0, T) ; \mathbb{R}^{2}\right)$. Hence they converge to $x$ in $C^{\beta}\left([0, T) ; \mathbb{R}^{2}\right.$ for $\beta<\alpha$ and such that $\mathcal{J}\left(x^{n} ; s, t\right) \underset{n \rightarrow \infty}{\longrightarrow} \mathcal{J}(x ; s, t)$ and $\mathcal{J}\left(z^{n} ; s, t\right) \underset{n \rightarrow \infty}{\longrightarrow} \mathcal{J}(x ; s, t)+\int_{0}^{T}[f, f]\left(x_{s}\right) d \phi_{s}$. Here $\phi$ is in $C^{\beta}\left([0, T) ; \mathbb{R}^{2}\right)$ for $\beta \in\left(\frac{2}{3}, 1\right]$ and $f \in$ $\operatorname{Lip}\left(\gamma ; \mathbb{R}^{2} \rightarrow \mathbb{R}^{m}\right)$ with $(\gamma+1) \beta>2$ such that $\phi$ approximates a loop. That is, let $y^{n}$ be a path starting and ending at the same point on the path $x$ piecewise of class $C^{1}$ such that for some fixed $\kappa$

1. $y_{k}^{n}:\left[t_{k}^{n}, t_{k+1}^{n}\right] \rightarrow \mathbb{R}^{2}$ with $y_{k}^{n}\left(t_{k}^{n}\right)=y_{k}^{n}\left(t_{k+1}^{n}\right)=x^{n}\left(t_{k}^{n}\right)$.

2. $\sup _{n=1, \ldots, k=0, \ldots, 2^{n}}\left\|y_{k}^{n}\right\|_{\frac{\beta}{2}}<\infty$,

3. uniformly in $n, k$, Area $\left(y_{k}^{n}\right)-\left(\phi\left(t_{k+1}^{n}\right)-\phi\left(t_{k}^{n}\right) \mid \leq C T^{\kappa} 2^{-n \kappa}\right.$,

where Area $\left(y_{k}^{n}\right)$ is the algebraic area of the loop $y_{k}^{n}$.

\subsubsection{Construction of the Integral by Approximations}

To solve this inconsistency with the limits with need to add some more information to our path. One way to do this is to choose a function $\phi$ so that we consider the space $C^{\alpha}\left([0, T) ; \mathbb{R}^{2} \times\right.$ $C^{2 \alpha}([0, T) ; \mathbb{R})$ equipped with the norm

$$
\|(x, \phi)\|=\|x\|_{\alpha}+\|\phi\|_{2 \alpha}
$$


such that the map

$$
\mathcal{J}(x, \phi):=\mathcal{J}(x)+\int[f, f]\left(x_{s}\right) d \phi_{s}
$$

is continuous. Denote by $L^{\alpha}\left([0, T], \mathbb{R}^{d}\right)$ the set of functions $x \in C^{\alpha}\left([0, T], \mathbb{R}^{d}\right)$ such that the linear approximation $\left\{x^{\Pi^{n}}\right\}_{n \in \mathbb{N}}$, where $\Pi^{n}$ is the dyadic partition of $[0, T]$ at level $n$, satisfy

$$
\mathcal{J}(x):=\lim _{n \in \mathbb{N}} \mathcal{J}\left(x^{\Pi^{n}}\right)
$$

exists in $C^{\alpha}\left([0, T], \mathbb{R}^{d}\right)$ and

$$
\left|\mathcal{J}\left(x_{\mid[s, t]}\right)-\mathcal{J}\left(x_{\mid[s, t]}^{\Pi^{n}}\right)\right| \leq K\left\|x-x^{\Pi^{n}}\right\|_{\alpha}|t-s|^{\alpha} .
$$

Let

$$
\mathcal{U}(x ; s, t):=\frac{1}{2} \int_{s}^{t}\left(x_{r}^{1}-x_{s}^{1}\right) d x_{r}^{2}-\frac{1}{2} \int_{s}^{t}\left(x_{r}^{2}-x_{s}^{2}\right) d x_{r}^{1}
$$

Now, we approximate

$$
J^{n}:=\sum_{k=0}^{2^{n}-1}\left(\int_{t_{k}^{n}+\frac{T}{2^{n+1}}}^{t_{k+1}^{n}} f\left(x_{s}^{n}\right) d x_{s}^{n}+[f, f]\left(x_{t_{k}^{n}}\right) \mathcal{U}\left(x^{n} ; t_{k}^{n}, t_{k}^{n}+\frac{T}{2^{n+1}}\right)\right)
$$

The path $\left(x^{1}, x^{2}, \mathcal{U}(x)\right)$ lives in three dimensions. For $u \in \mathbb{R}$ let

$$
\mathcal{C}(x, u ; t):=\left(x_{t}^{1}, x_{t}^{2}, t+\mathcal{U}(x ; 0, t)\right)
$$

for $t \in[0, T]$. Let us define a new group operator by

$$
a \boxplus b=\left(a^{1}+b^{1}, a^{2}+b^{2}, a^{3}+b^{3}+\frac{1}{2}\left[\begin{array}{l}
a^{1} \\
a^{2}
\end{array}\right] \wedge\left[\begin{array}{l}
b^{1} \\
b^{2}
\end{array}\right]\right) .
$$

Now, we equip $\mathbb{R}^{3}$ with this new group operation $\boxplus$ and call this new group $A\left(\mathbb{R}^{2}\right)$. Then we let the Lie commutator

$$
[a, b]=a \boxplus b-b \boxplus a=\left(0,0, \frac{1}{2}\left[\begin{array}{l}
a^{1} \\
a^{2}
\end{array}\right] \wedge\left[\begin{array}{l}
b^{1} \\
b^{2}
\end{array}\right]\right)
$$

So the group $A\left(\mathbb{R}^{2}\right)$ is non-commutative.

Lemma 18.5. The space $\left(A\left(\mathbb{R}^{2}\right),[\cdot, \cdot]\right)$ is a Lie algebra.

Proof. See Lemma 4 Lejay [2009].

Lemma 18.6. The space $\left(A\left(\mathbb{R}^{2}\right),[\cdot, \cdot]\right)$ is a Lie group.

Proof. See Lemma 5 Lejay [2009]. 
Definition 18.7. For a continuous path $x$ with values in $\mathbb{R}^{2}$ a continuous path $\mathbf{x}$ with values in $A\left(\mathbb{R}^{2}\right)$ such that $x=\left(\mathbf{x}^{1}, \mathbf{x}^{2}\right)$ is called a path lying above $x$ or $a$ lift of $x$.

Let $x$ and $y$ be two smooth path lifted by $\mathbf{x}=\mathcal{C}(x, 0)$ and $\mathbf{y}=\mathcal{C}(y, 0)$. Then it is easy to see that the usual concatenation of paths $\mathbf{x} \circ \mathbf{y}$ is different from $\mathcal{C}(x \circ y, 0)$. Therefore we define a new concatenation of two paths $\mathbf{x}:[0, T] \rightarrow A\left(\mathbb{R}^{2}\right)$ and $\mathbf{y}:[0, S] \rightarrow A\left(\mathbb{R}^{2}\right)$ by:

$$
(\mathbf{x} \boxminus \mathbf{y})_{t}:= \begin{cases}\mathbf{x}_{t} ; & \text { if } t \in[0, T], \\ \mathbf{x}_{T} \boxplus\left(\left(-\mathbf{y}_{0}\right) \boxplus \mathbf{y}_{t-T}\right) ; & \text { if } t \in[T, T+S] .\end{cases}
$$

Now we construct a partition of $x$ by linear interpolation. Let $\Pi^{n}$ be the dyadic partition at level $n$ and

$$
\theta_{k}^{n}=\left(\left(-\mathbf{x}_{t_{k+1}^{n}} \boxplus \mathbf{x}_{t_{k}^{n}}\right)^{3}\right.
$$

Let $\Phi^{n}=\left\{y_{k}^{n}\right\}_{k=0, \ldots, 2^{n}-1}$ with $y_{k}^{n}:\left[t_{k}^{n}, t_{k+1}^{n}\right] \rightarrow \mathbb{R}^{2}$ and

$$
y_{k}^{n}(t)=\sqrt{\frac{\left|\theta_{k}^{n}\right|}{\pi}}
$$

Then, let

$$
x_{t}^{n}=x^{\Pi^{n}} \bowtie \Phi^{n}\left(\frac{t}{2}\right)
$$

for $t \in[0, T]$ and

$$
\mathbf{x}^{n}=\left(x^{n}, \mathbf{x}_{0}^{3}+\mathcal{U}\left(x^{n}, 0, \cdot\right)\right) .
$$

The we can construct the integral

$$
\mathcal{J}(\mathbf{x}):=\mathcal{J}(x):=\int_{\left.x\right|_{[0, \cdot]}} f .
$$

Proposition 18.8. Let $\mathbf{x} \in C^{\alpha}\left([0, T], A\left(\mathbb{R}^{2}\right)\right)$ with $\alpha \in\left(\frac{1}{3}, \frac{1}{2}\right]$ and $f$ a differential form in Lip $\left(\gamma, \mathbb{R}^{2} \rightarrow \mathbb{R}\right)$ with $\gamma>\frac{1}{\alpha}-1$. Let $\{x\}_{n \in \mathbb{N}}$ be as in (18.3) - (18.6). Then $\left\{\mathcal{J}\left(x^{n}\right)\right\}_{n \in \mathbb{N}}$ has a unique limit in $\left(C^{\alpha}([0, T], \mathbb{R}),\|\cdot\|_{\beta}\right)$ for all $\beta<\alpha$, denoted $\mathcal{J}(\boldsymbol{x})$.

Proof. See proposition 3 in Lejay [2009].

Let $H$ denote the Heisenberg group, that is

$$
H=\left\{\left[\begin{array}{lll}
1 & a & c \\
0 & 1 & b \\
0 & 0 & 1
\end{array}\right] \mid a, b, c \in \mathbb{R}\right\}
$$

Further, construct the mapping

$$
\Phi(x)=\left[\begin{array}{lll}
0 & a & c \\
0 & 0 & b \\
0 & 0 & 0
\end{array}\right]
$$


for $x=(a, b, c) \in A\left(\mathbb{R}^{2}\right)$. Then $\phi$ is a one-to-one map between $A\left(\mathbb{R}^{2}\right)$ and $T_{I d} H$. Here $T_{a} M$ of a manifold $M$ is the tangent bundle at $a$. further

$$
\Phi([x, y])=[\Phi(x), \Phi(y)]
$$

for all $x, y \in A\left(\mathbb{R}^{2}\right)$. So $\Phi$ is actually a Lie algebra isomorphism. Thus with the exponential map, exp, we may identify a path $\mathbf{X} \in A\left(\mathbb{R}^{2}\right)$ with a path $\mathbf{Y}=\exp (\mathbf{X})$ in the Heisenberg group. Now, we want to construct a space that contains the Heisenberg group. This we do using the tensor algebra, $T\left(\mathbb{R}^{2}\right)=\mathbb{R} \otimes \mathbb{R}^{2} \otimes\left(\mathbb{R}^{2} \otimes \mathbb{R}^{2}\right)$. For $\zeta \in\{0,1\}$, we denote by $T_{\zeta}\left(\mathbb{R}^{2}\right)$ the subset of $T\left(\mathbb{R}^{2}\right)$ defined by

$$
T_{\zeta}\left(\mathbb{R}^{2}\right)=\left\{\left(\zeta, x^{1}, x^{2}\right) \mid x^{1} \in \mathbb{R}^{2}, c^{2} \in \mathbb{R}^{2} \otimes \mathbb{R}^{2}\right\}
$$

It can relatively easily be shown that the space $\left(T_{1}\left(\mathbb{R}^{2}\right), \otimes\right)$ is a non-commutative group. Define the bracket of $x, y \in T\left(\mathbb{R}^{2}\right)$ by

$$
[x, y]=x \otimes y-y \otimes x
$$

Let

$$
g\left(\mathbb{R}^{2}\right)=\left\{x \in T_{0}\left(\mathbb{R}^{2}\right) \mid x=x^{1}+x^{a}\left[e_{1}, e_{2}\right], x^{1} \in \mathbb{R}^{2}, x^{a} \in \mathbb{R}\right\} .
$$

Note that the tensor algebra $T\left(\mathbb{R}^{2}\right)$ is actually a truncated tensor algebra where only the 3 first levels are non-zero. This gives us that the exponential map on $T_{0}\left(\mathbb{R}^{2}\right)$ is given by

$$
\exp (x)=1+x^{1}+x^{2}+\frac{1}{2} x^{1} \otimes x^{1}
$$

Now let $G\left(\mathbb{R}^{2}\right)=\exp \left(g\left(\mathbb{R}^{2}\right)\right)$. Then $G\left(\mathbb{R}^{2}\right)$ is a subgroup of $\left(T_{1}\left(\mathbb{R}^{2}\right), \otimes\right)$ and exp is a group isomorphism from $\left(g\left(\mathbb{R}^{2}\right), \boxplus\right)$ to $\left(G\left(\mathbb{R}^{2}\right), \otimes\right)$. All of the above extends to arbitrary Euclidean spaces $V$. The space of all smooth rough paths is denoted by $\Omega_{p}^{\infty}(V)$ and is endowed with the $p$-variation distance.

\subsection{Rough Path Differential Equations}

The following important theorem allows us to integrate against rough paths.

Theorem 18.9 (T.Lyons). Let $p \geq 1$ and let $f: \mathbb{R} \times \mathbb{R}^{n} \rightarrow L\left(\mathbb{R}^{d}, \mathbb{R}^{n}\right)$ be a differentiable function with bounded derivatives up to degree $[p]+1$. Denote by $F(\mathbf{W}, x) \in \Omega_{p}^{\infty}\left(\mathbb{R}^{n}\right)$ the smooth rough path associated with the unique solution $Y$ to the differential equation:

$$
\begin{cases}d Y_{t} & =f\left(t, Y_{t}\right) d W_{t} \\ Y_{0} & =x\end{cases}
$$

where $W$ is a continuous path in $\mathbb{R}^{d}$ with finite variation and $\mathbf{W}$ is its associated smooth rough path. Then the map (called the Itô map determined by the above differential equation) is continuous from $\Omega_{p}^{\infty}\left(\mathbb{R}^{d}\right)$ to $\Omega_{p}^{\infty}\left(\mathbb{R}^{n}\right)$ ( with respect to the p-variation distance). Therefore, there is a unique extension of the Itô map $F(\mathbf{W}, x)$ to the space $\Omega_{p}^{\infty}\left(\mathbb{R}^{d}\right)$ of all geometric rough space. 


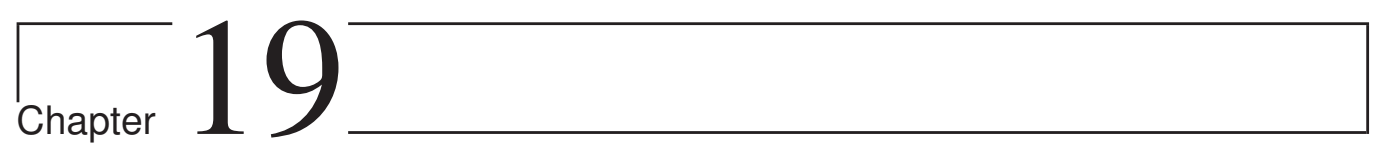

\section{Chaos Expansions and the Malliavin Derivative}

Malliavin Calculus is an infinite-dimensional differential calculus on the Wiener space. It extends the calculus of variations from functions to stochastic processes. Malliavin ideas led to a proof that Hörmander's condition implies the existence and smoothness of a density for the solution of a stochastic differential equation. The calculus allows integration by parts with random variables. This operation is used in mathematical finance to compute the sensitivities of many financial derivatives.

This is a very short introduction to the rich world of the Malliavin calculus. We refer the reader to the books Nualart [2006] and Nunno et al. [2009] for more information on Malliavin calculus.

\subsection{Wiener processes}

Assume we are given a Hilbert space $H$.

Definition 19.1 (Isonormal Gaussian Processes). A stochastic process $W=\{W(h)\}_{h \in H}$ is an isonormal Gaussian process if $W$ is a centred Gaussian family of random variables such that

$$
E[W(h) W(g)]=\langle h, g\rangle_{H}
$$

for all $h, g \in H$.

\subsubsection{Hermite Polynomial, Function and the Wiener Chaos}

Let us define the Hermite polynomials and the Hermite functions.

Definition 19.2 (Hermite Polynomials and Functions).

1.

$$
H_{n}(x)=(-1)^{n} e^{\frac{x^{2}}{2}} \frac{d^{n}}{d x^{n}}\left(e^{-\frac{x^{2}}{2}}\right),
$$

for $n=1,2, \ldots$ is the Hermite polynomial. 
2.

$$
\xi_{n}(x)=\frac{1}{\pi^{\frac{1}{4}}((n-1) !)^{\frac{1}{2}}} H_{n-1}(\sqrt{2} x) e^{-\frac{x^{2}}{2}}
$$

for $n=1,2, \ldots$, is the Hermite function.

We have that $\xi_{n}(x) \in \mathcal{S}(\mathbb{R})$. Let $\mathcal{J}$ be the set of all multi-indices $\alpha=\left(\alpha_{1}, \alpha_{2}, \ldots\right)$ of finite length $l(\alpha)=\max \left\{i ; \alpha_{i} \neq 0\right\}$, with $\alpha_{i} \in \mathbb{N}_{0}$ for all $i$. For $\alpha \in \mathcal{J}$ with length $l(\alpha)=n$, let $\alpha !=a_{1} ! a_{2} ! \cdots a_{n} !$ and $|\alpha|=\alpha_{1}+\cdots+\alpha_{n}$.

Definition $19.3\left(\mathcal{H}_{n}\right)$. For each $n \geq 1$ we will denote by $\mathcal{H}_{n}$ the closed linear subspace of $L^{2}(\Omega, \mathcal{F}, \mathcal{P})$ generated by the random variables

$$
\left\{H_{n}(W(h)), h \in H,\|h\|_{H}=1\right\}
$$

It is easy to see that $\mathcal{H}_{n}$ and $\mathcal{H}_{m}$ are orthogonal whenever $n \neq m$. The space $\mathcal{H}_{n}$ is called the Wiener chaos of order $n$.

Theorem 19.4. The space $L^{2}(\Omega, \mathcal{F}, \mathcal{P})$ can be decomposed as follows;

$$
L^{2}(\Omega, \mathcal{F}, \mathcal{P})=\otimes_{n=0}^{\infty} \mathcal{H}_{n}
$$

\subsubsection{The Derivative Operator}

Let $\mathcal{S}$ denote the class of smooth random variables of the form

$$
F=f\left(W\left(h_{1}\right), \ldots, W\left(h_{n}\right)\right),
$$

for $f \in C_{p}^{\infty}\left(\mathbb{R}^{n}\right), h_{1}, \ldots, h_{n} \in H$ and $n \geq 1$.

Definition 19.5. The operator $D$ on $\mathcal{S}$ is defined by

$$
D F=\sum_{i=1}^{n} \partial_{i} f\left(W\left(h_{1}\right), \ldots, W\left(h_{n}\right)\right) h_{i} .
$$

Lemma 19.6. Suppose that $F$ is a smooth random variable and $h \in H$. Then

$$
E\left(\langle D F, h\rangle_{H}\right)=E(F W(h))
$$

Proof. See Lemma 1.2.1 in Nualart [2006].

Lemma 19.7. Suppose that $F$ and $G$ are smooth random variables and $h \in H$. Then

$$
\left.E\left(G\langle D F, h\rangle_{H}\right)=E\left(-F\langle D G, h\rangle_{H}\right)+F G W(h)\right) .
$$

Proof. See Lemma 1.2.2 in Nualart [2006].

Proposition 19.8. The operator $D$ is closable from $L^{p}(\Omega)$ to $L^{p}(\Omega, H)$.

Proof. See Proposition 1.2.1 in Nualart [2006]. 


\subsubsection{The Divergence Operator}

Definition 19.9. The divergence operator, $\delta$, is defined as the adjoint of the operator $D$.

From the definition of $\delta$ we have that it is an unbounded operator on $L^{2}(\Omega, H)$ with values on $L^{2}(\Omega)$ such that:

1. The domain of $\delta, D o m \delta$, is the set of all $H$-valued square integrable random variables $u \in L^{2}(\Omega, H)$ such that

$$
\left|E\left(\langle D F, u\rangle_{H}\right)\right| \leq c\|F\|_{2},
$$

for all $F \in \mathbb{D}^{1,2}$, where $c$ is a constant depending on $u$.

2. If $u$ belongs to $D o m \delta$, the $\delta(u)$ is the element of $L^{2}(\Omega)$ characterized by

$$
E(F \delta(u))=E\left(\langle D F, u\rangle_{H}\right)
$$

for all $F \in \mathbb{D}^{1,2}$.

\subsection{Lévy processes}

For notational convenience, we assume in this subsection $d=1$. Consider $\Omega=\mathcal{S}\left(\mathbb{R}^{d}\right)=\mathcal{S}(\mathbb{R})$, the space of tempered distributions on $\mathbb{R}$. Then we know from the Bochner-Minlos-Sazonov theorem (see e.g. Kallianpur and Xiong [1995]) that there exists a probability measure $\mu$, such that

$$
\int_{\Omega} e^{i\langle\omega, f\rangle} \mu(d \omega)=\exp \left(\int_{\mathbb{R}} \Psi(f(x)) d x\right),
$$

for $f \in S(\mathbb{R})$, where $\Psi$ is the characteristic exponent given by

$$
\Psi(u)=\int_{\mathbb{R}}\left(e^{i u z}-1-i u z\right) \nu(d z),
$$

where $\langle\omega, f\rangle$ denotes the action of $\omega \in \mathcal{S}^{\prime}(\mathbb{R})$ (Schwartz distribution space) on $f \in \mathcal{S}(\mathbb{R})$ and where $\nu$ is a Lévy measure. The triple $(\Omega, \mathcal{F}, \mu)$ is called the (pure jump) Lévy white noise probability space.

From now on we assume a square integrable Lévy process $L_{t}, t \geq 0$ with Lévy measure $\nu$ constructed on $(\Omega, \mathcal{F}, \mu)$.

\subsubsection{Malliavin Derivative for Lévy processes}

In what follows we want to use the chaos representation property of a square integrable Lévy process to define the Malliavin derivative with respect to such processes. To this end we need some notation:

Let us denote by $\mathcal{J}$ the set of all finite multi-indices $\alpha=\left(\alpha_{1}, \alpha_{2}, \ldots, \alpha_{m}\right), m \in \mathbb{N}_{0}$ of nonnegative integers $a_{i}, i=1, \ldots, m$, and define $|\alpha|:=\alpha_{1}+\ldots \alpha_{m}$. Further, let $e_{i}, i \geq 1$ be an 
orthonormal basis of $L^{2}(\lambda \times \nu)\left(\lambda\right.$ Lebesgue measure) and let for $\alpha=\left(\alpha_{1}, \alpha_{2}, \ldots, \alpha_{m}\right) \in \mathcal{J}$

$$
\begin{aligned}
& H_{\alpha}=\int_{\mathbb{R}} \int_{\mathbb{R}_{0}} \cdots \int_{\mathbb{R}} \int_{\mathbb{R}_{0}} e_{1}^{\otimes \alpha_{1}} \hat{\otimes} \cdots \hat{\otimes} e_{m}^{\otimes \alpha_{m}}\left(\left(s_{1}, z_{1}\right), \ldots,\left(s_{m}, z_{m}\right)\right) \\
& \tilde{N}\left(d s_{1}, d z_{1}\right) \cdots \widetilde{N}\left(d s_{m}, d z_{m}\right),
\end{aligned}
$$

where $\otimes$ and $\hat{\otimes}$ denotes the tensor product and the symmetrized tensor product, respectively.

Then $\left\{H_{\alpha}: \alpha \in \mathcal{J}\right\}$ forms an orthogonal basis of $L^{2}(\mu)$ :

Theorem 19.10 (Chaos expansion). Any $X \in L^{2}(\mu)$ has the unique chaos decomposition of the form

$$
X=\sum_{\alpha \in \mathcal{J}} c_{\alpha} H_{\alpha}
$$

with $c_{\alpha} \in \mathbb{R}$. Moreover

$$
\|X\|_{L^{2}(\mu)}^{2}=\sum_{\alpha \in \mathcal{J}} \alpha ! c_{\alpha}^{2}
$$

where

$$
\alpha !:=\alpha_{1} !, \alpha_{2} !, \ldots, \alpha_{m} !
$$

for $\alpha=\left(\alpha_{1}, \alpha_{2}, \ldots, \alpha_{m}\right)$.

We are now ready to define the Malliavin derivative. The Malliavin derivative of a square integrable functional $X$ of a pure jump Lévy process $L$ with chaos expansion

$$
X=\sum_{\alpha \in \mathcal{J}} c_{\alpha} H_{\alpha}
$$

is defined by

$$
D_{t, z} X=\sum_{\beta \in \mathcal{J}} \sum_{i \in \mathbb{N}}\left(c_{\beta+\epsilon_{i}}\left(\beta_{i}+1\right)\right) e_{i}(t, z) H_{\beta},
$$

provided $X$ belongs to the domain $\mathbb{D}^{1,2} \subset L^{2}(\mu)$ given by

$$
\begin{gathered}
\mathbb{D}^{1,2}:=\left\{X \in L^{2}(\mu)\right. \text { with chaos expansion (19.4): } \\
\left.\sum_{\beta \in \mathcal{J}} \sum_{i \in \mathbb{N}}\left(c_{\beta+\epsilon_{i}}\left(\beta_{i}+1\right)\right)^{2} \beta !<\infty\right\}
\end{gathered}
$$

where $\epsilon_{i}:=(0,0, \ldots, 0,1,0, \ldots, 0)$ with 1 in the $i$-th position. 


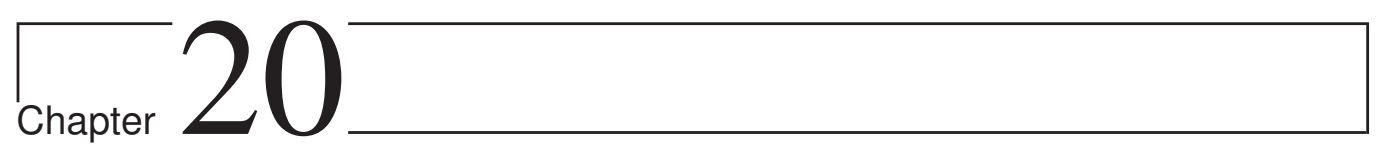

\section{White Noise}

For many problems we need a wider definition of a function or a process. As in pde theory we often use generlaized processes to find solution of differential equations. Defining a generalized stochastic process in stochastic analysis is not of much help since its sample path property is lost and nonlinear functionals of white noise cannot be defined. To overcome these difficulties, T. Hida introduced in 1975 the theory of white noise. This theory enables us to define the white noise $d B(t) / d t$ as a generalized function on an infinite dimensional space. This white noise framework is a flexible framework whith many applications.

This chapter is based on Holden et al. [2010], Kuo [1996], Obata [1994], Hida et al. [1993] and Rudin [1991].

\subsection{Topological Vector Spaces}

In this section we are going to need some background theory from infinite dimensional measures and process. Since there is a "thorough" review in Chapter 23 we are just listing some basic theory here. A norm, $\|\cdot\|$, on $H$ is called measurable if for any $\epsilon>0$, there exists a finite dimensional orthogonal projection $P_{\epsilon}$ such that for any finite dimensional orthogonal projection $P$ with $P P_{\epsilon}=0$, we have

$$
\mu_{P}\{x \in P H,\|x\|>\epsilon\}<\epsilon,
$$

where $\mu_{P}$ is the standard Gaussian measure on $P H$. The link between the above definition and the theory in Chapter 23 is that an inner product norm, $\|\cdot\|$, is measurable if and only if $\|x\|=|T x|$ for an injective Hilbert-Schmidt operator $T$ on $H$. Let $B$ be the completion of $H$ with respect to a measurable norm, $\|\cdot\|$. The pair $(H, B)$ is called an abstract Wiener space.

Theorem 20.1. Let $(H, B)$ be an abstract Wiener space and $i$ the inclusion map from $H$ into $B$. Then the cylindrical measure $\mu \circ i$ on $B$ is $\sigma$-additive.

We now have from the Riesz representation theorem that we have a continuous inclusion $B^{*} \subset H \subset B$. As usual, extend the dual bilinear pairing on $\left(B, B^{*}\right)$ to $\left(H, B^{*}\right)$ by continuity. Now we consider a topological vector space, $V$, over $\mathbb{C}$, with the topology given by a family $\left\{|\cdot|_{n}, n \geq 1\right\}$ of inner product norms. For $u, v \in V$, define

$$
d(u, v)=\sum_{n=1}^{\infty} \frac{1}{2^{n}} \frac{|u-v|_{n}}{1+|u-v|_{n}} .
$$


A topological vector space, $V$, with a family of inner product norms is called a countably-Hilbert space if it is complete with respect to its topology. Without loss of generality we can assume that the norms are increasing. Let $V_{n}$ be the completion of $V$ in the norm $|\cdot|$. Then $V_{n}$ is a Hilbert space and we have

$$
V \subset \cdots \subset V_{n} \subset \cdots \subset V_{1} .
$$

Conversely for a sequence of Hilbert spaces, $\left\{U_{n}\right\}$, such that $U_{n+1}$ is continuously embedded in $U_{n}$. Then if we endow $U:=\cap_{n=1}^{\infty} U_{n}$ with the projective limit topology, it is called the projective limit. For the (topological) dual spaces we have that

$$
V_{1}^{*} \subset \cdots \subset V_{n}^{*} \subset \cdots \subset V^{*}
$$

Then there are several choices of topology to assign to $V^{*}$ :

1. the weak topology;

2. the strong topology;

3. and the inductive limit topology.

Let $V$ be a countably-Hilbert space. If for any $n$, there exists $m \geq n$ such that the inclusion map from $V_{m}$ into $V_{n}$ is a Hilbert-Schmidt operator, then $V$ is called nuclear. On nuclear spaces Minlos theorem applies.

\subsection{White Noise Space}

A function $\xi$ on $\mathbb{R}$ is called rapidly decreasing if it is smooth and for all nonnegative integers $n, k$ :

$$
\left|x^{n} \xi^{(k)}(x)\right| \rightarrow 0
$$

as $|x| \rightarrow \infty$. The space of all rapidly decreasing function is called the Schwartz space, $\mathcal{S}(\mathbb{R})$. Define a family of norms, $|\cdot|_{n, k}$ on $\mathcal{S}(\mathbb{R})$ by

$$
|\xi|_{n, k}=\left(\int_{\mathbb{R}}\left|x^{n} \xi^{(k)}(x)\right|^{2} d x\right)^{\frac{1}{2}} .
$$

The topology generated by this family makes $\mathcal{S}(\mathbb{R})$ into a nuclear space. We also have the following Gel'fand triple

$$
\mathcal{S}(\mathbb{R}) \subset L^{2}(\mathbb{R}) \subset \mathcal{S}^{*}(\mathbb{R})
$$

Define a function on $\mathcal{S}(\mathbb{R})$ by

$$
C(\xi)=e^{-\frac{1}{2}|\xi|_{0}^{2}} .
$$

Then, Minlos theorem apply and we have a unique probability measure, $\mu$, on the dual space $\mathcal{S}^{*}(\mathbb{R})$. 


\subsubsection{Construction of the White Noise Space}

We are going to construct the white noise space from a real separable Hilbert space $E$ with a given norm, $|\cdot|_{0}$. Assume we have an operator, $A$, on $E$ such that we have an orthonormal basis $\left\{\xi_{i}\right\}_{i=1,2, \ldots}$, for $E$ satisfying the following:

1. $A \xi_{i}=\lambda_{i} \xi_{i}$;

2. $1 \leq \lambda_{1} \leq \lambda_{2} \leq \cdots$;

3. $\sum_{i=1}^{\infty} \frac{1}{\lambda_{i}^{\alpha}}<\infty$, for some constant $\alpha>0$.

Now, we can construct a family of norms $|\zeta|_{p}=\left|A^{p} \zeta\right|_{0}$. If we now let

$$
\varepsilon_{p}:=\left\{\zeta \in E,|\zeta|_{p}<\infty\right\}
$$

then is is easy to see that $\mathcal{E}_{p} \subset \mathcal{E}_{q}, p \geq q$, and that the inclusion map $\mathcal{E}_{p+\frac{\alpha}{2}} \hookrightarrow \mathcal{E}_{q}, p \geq 0$, is Hilbert-Schmidt. Thus $\mathcal{E}$ is nuclear and we can apply Minlos theorem to get a measure $\mu$ on $\mathcal{E}^{*}$. By utilizing the Wiener-Itô expansion (see section 19.1.1) or by using the same method as above, we get the space of stochastic test functions and stochastic distributions.

1. Let $\left(L^{2}\right)$ denote the complex Hilbert space $L^{2}\left(\mathcal{E}^{*}, \mu\right)$. We have that every element $\phi \in\left(L^{2}\right)$ can be expressed uniquely as

$$
\phi=\sum_{n=0}^{\infty} I_{n}\left(f_{n}\right),
$$

where $f_{n} \in E_{C}^{\hat{\otimes} n}$. Further,

$$
\|\phi\|_{0}^{2}=\sum_{n=0}^{\infty} n !\left|f_{n}\right|_{0}^{2} .
$$

We apply the same method as above by using the second quantization operator $\Gamma(A)$ of $A$ defined densely on $\left(L^{2}\right)$ by:

$$
\Gamma(A) \phi=\sum_{n=0}^{\infty} I_{n}\left(A^{\otimes n} f_{n}\right),
$$

for $\phi=\sum_{n=0}^{\infty} I_{n}\left(f_{n}\right)$. We now get a new Gel'fand triple by defining

$$
\|\phi\|_{p}=\left\|\Gamma(A)^{p} \phi\right\|_{0}
$$

and letting

$$
\left(\varepsilon_{p}\right):=\left\{\zeta \in\left(L^{2}\right),\|\phi\|_{p}<\infty\right\}
$$

2. The second method by kondratiev and Streit uses the representation as a cornerstone in defining the stochastic test function space and stochastic distribution space. For $0<\beta<$ 
1, define a norm by

$$
\|\phi\|_{p, \beta}=\left(\sum_{n=0}^{\infty}(n !)^{1+\beta}\left|\left(A^{p}\right)^{\otimes n} f_{n}\right|_{0}^{2}\right)^{\frac{1}{2}}
$$

and let

$$
\left(\mathcal{E}_{p}\right)_{\beta}:=\left\{\zeta \in\left(L^{2}\right),\|\phi\|_{p, \beta}<\infty\right\} .
$$

Let

$(\varepsilon)_{\beta}$

be the projective limit of $\left\{\left(\varepsilon_{p}\right)_{\beta}\right\}_{p \geq 0}$ and

$$
(\varepsilon)_{\beta}^{*}
$$

the dual space of $(\varepsilon)_{\beta}$.

\subsection{Wick product}

We now need a operation that in allows us to "multiply" two stochastic distributions. Therfore we introduce the Wick product.

Definition 20.2. For two elements given by

$$
F=\sum_{\alpha} a_{\alpha} H_{\alpha}
$$

and

$$
G=\sum_{\alpha} b_{\alpha} H_{\alpha}
$$

both in $(\varepsilon)_{1}^{*}$. Then the Wick product is defined by

$$
F \diamond G=\sum_{\alpha, \beta} a_{\alpha} b_{\beta} H_{\alpha+\beta}
$$

Definition 20.3. A function $Z(t): \mathbb{R} \rightarrow(\mathcal{E})^{*}$ is called $(\mathcal{E})^{*}$-integrable if

$$
\langle Z(t), f\rangle \in L^{1}(\mathbb{R}, d t)
$$

for all $f \in(\mathcal{E})$. The $(\mathcal{E})^{*}$-integral of $Z(t)$ is the $(\mathcal{E})^{*}$-element such that

$$
\left\langle\int_{\mathbb{R}} Z(t) d t, f\right\rangle=\int_{\mathbb{R}}\langle Z(t), f\rangle d t
$$


Theorem 20.4. If $Y(t)=\sum_{\alpha} c_{\alpha}(t) H_{\alpha}$ is a Skorohod-integrable process and $a, b \in \mathbb{R}$, then $Y(t) \diamond W(t)$ is $(\mathcal{E})^{*}$-integrable over $[a, b]$ and

$$
\int_{a}^{b} Y(t) d B(t)=\int_{a}^{b} Y(t) \diamond W(t) d t .
$$

Proof. See theorem 2.5.9 in Holden et al. [2010].

Now consider a function $\phi \in\left(L^{2}\right)$ and its $\mu$-convolution

$$
\mu \phi(h)=\int_{\mathcal{E}^{*}} \phi(x+h) d \mu(x),
$$

for $h \in E$. By using the translation formula for $\frac{d \mu_{h}}{d \mu}$ we have that

$$
\mu \phi(h)=\int_{\mathcal{E}^{*}} \phi(x) e^{\langle x, h\rangle-\frac{1}{2}|h|_{0}^{2}} d \mu(x)
$$

We now want to generalize this to generalized functions and to achieve that we need to define a generalization of exp. Let

$$
: e^{\langle\cdot, h\rangle}:=e^{\langle\cdot, h\rangle-\frac{1}{2}|h|_{0}^{2}}
$$

for $h \in E$. Then consider its Wiener-Itô decomposition

$$
: e^{\langle\cdot, h\rangle}:=\sum_{n=0}^{\infty} \frac{1}{n !}\left\langle: x^{\otimes n}:, h^{\otimes n}\right\rangle .
$$

Then we can extend the definition and define the $S$-transform using it.

Definition 20.5. The S-transform, $S \Phi$, of a generalized function $\Phi \in(\mathcal{E})_{\beta}^{*}$ is defined as

$$
S \Phi(\zeta)=\left\langle\Phi,: e^{\langle\cdot, \zeta\rangle}:\right\rangle_{(\varepsilon)^{*}}
$$

for $\zeta \in \mathcal{E}_{C}$.

Definition 20.6. the Wick tensor : $x^{\otimes n}$ : of an element $x$ in $\mathcal{E}^{\prime}$ is defined as

$$
: x^{\otimes n}:=\sum_{k=0}^{[n / 2]}\left(\begin{array}{c}
n \\
2 k
\end{array}\right)(2 k-1) ! !: x^{\otimes(n-2 k)}: \hat{\otimes} \tau^{\otimes k}
$$

where $\tau$ is the trace operator.

\subsection{Differential operators}

Let us define a operator differential operator $D_{y}$ on $(\mathcal{E})_{\beta}$ by: 
Definition 20.7. For $\phi(x)=\sum_{n=0}^{\infty}\left\langle: x^{\otimes(n-1)}:, f_{n}\right\rangle$

$$
D_{y} \phi(x)=\sum_{n=0}^{\infty} n\left\langle: x^{\otimes(n-1)}:,\left\langle y, f_{n}\right\rangle\right\rangle .
$$

In the case of the Gel'fand triplets $\mathcal{S} \subset L^{2}(T, \nu) \subset \mathcal{S}^{*}$ we call $D_{\delta_{t}}$ the white noise differential operator and we denote it by $\partial_{t}$. Let us also define

$$
(\nabla \phi)(t, x):=\partial_{y} \phi(x)
$$

where we call $\nabla \phi$ the gradient of $\phi$. Thus $\nabla: D(\nabla) \subset\left(L^{2}\right) \rightarrow L^{2}\left(T \times \mathcal{S}^{*}\right)$. 


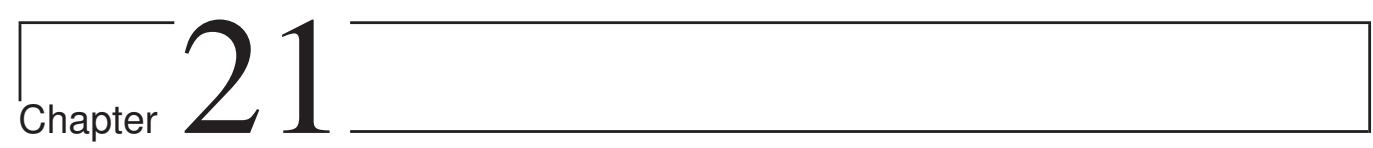

\section{Fractional Derivatives and Integrals}

The possibility of taking real number powers or complex number powers of the differentiation operator

$$
D=\frac{d}{d x},
$$

and the integration operator is of great interest. What would for example be a meaningful interpretation of

$$
\sqrt{D}=D^{\frac{1}{2}} ?
$$

We would like an extension to the differential operator which is a semigroup of powers $D^{a}$ forming a continuous semigroup with parameter a, from which the original discrete semigroup $D^{n}$ for integer $n$ can be recovered as a subgroup.

This chapter is based partly on Hilfer [2008].

Let

$$
K_{ \pm}^{\alpha}(x):=\theta( \pm x) \frac{( \pm x)^{\alpha-1}}{\Gamma(\alpha)}
$$

for $\alpha>0$ where

$$
\theta(x)= \begin{cases}1, & x>0 \\ 0, & x \leq 0\end{cases}
$$

is the Heaviside step function. 


\subsection{Riemann-Liouville Fractional Integrals}

Definition 21.1. Let $-\infty \leq a<x<b \leq \infty$. The Riemann-Liouville fractional integral of order $\alpha>0$ with lower limit $a$ is defined for locally integrable functions $f:[a, b] \rightarrow \mathbb{R}$ by

$$
\left(I_{a^{+}}^{\alpha} f\right)(x)=\frac{1}{\Gamma(\alpha)} \int_{a}^{x}(x-y)^{\alpha-1} f(y) d y
$$

The Riemann-Liouville fractional integral of order $\alpha>0$ with upper limit $b$ is defined by

$$
\left(I_{b^{-}}^{\alpha} f\right)(x)=\frac{1}{\Gamma(\alpha)} \int_{x}^{b}(x-y)^{\alpha-1} f(y) d y
$$

\subsection{Fractional Integrals of Weyls Type}

For $\alpha \in(0,1)$ let

$$
\left(I_{-}^{\alpha} f\right)(x):=\frac{1}{\Gamma(\alpha)} \int_{x}^{\infty} f(t)(t-x)^{\alpha-1} d t=\frac{1}{\Gamma(\alpha)} \int_{0}^{\infty} f(x+t) t^{\alpha-1} d t
$$

and

$$
\left(I_{+}^{\alpha} f\right)(x):=\frac{1}{\Gamma(\alpha)} \int_{-\infty}^{x} f(t)(t-x)^{\alpha-1} d t=\frac{1}{\Gamma(\alpha)} \int_{0}^{\infty} f(x-t) t^{\alpha-1} d t
$$

\subsection{Fractional Integration by Parts}

For $f \in L^{p}([a, b]), g \in L^{q}([a, b])$ with $\frac{1}{p}+\frac{1}{q} \leq 1, p, q \geq 1$ and $p, q \neq 1$ for $\frac{1}{p}+\frac{1}{q}=1+\alpha$ we have

$$
\int_{a}^{b} f(x)\left(I_{a^{+}}^{\alpha} g\right)(x) d x=\int_{a}^{b} g(x)\left(I_{a^{+}}^{\alpha} f\right)(x) d x .
$$

For Weyls fractional integrals we have

$$
\int_{-\infty}^{\infty} f(x)\left(I_{+}^{\alpha} g\right)(x) d x=\int_{-\infty}^{\infty} g(x)\left(I_{-}^{\alpha} f\right)(x) d x .
$$

\subsection{Riemann-Liouville Fractional Derivatives}

Riemann defined the fractional derivative as

Definition 21.2. Let $-\infty \leq a<x<b \leq \infty$. The Riemann-Liouville fractional derivative of order $0<$ alpha $<1$ with lower limit a (upper limit b) is defined for functions $f \in L^{1}([a, b])$ 
such that $f * K^{1-\alpha} \in W^{1,1}([a, b])$ as

$$
\left(D_{a \pm}^{\alpha} f\right)(x)= \pm \frac{d}{d x}\left(I_{a \pm}^{1-\alpha} f\right)(x)
$$

\subsection{Fractional Derivatives of Marchaud's Type}

For $\alpha \in(0,1)$ and $\epsilon>0$,let

$$
\left(D_{ \pm, \epsilon}^{\alpha} f\right)(x):=\frac{\alpha}{\Gamma(1-\alpha)} \int_{\epsilon}^{\infty} \frac{f(x)-f(x \mp t)}{t^{\alpha+1}} d t
$$

Then we define

$$
\left(D_{ \pm}^{\alpha} f\right)(x):=\lim _{\epsilon \rightarrow 0^{+}}\left(D_{ \pm, \epsilon}^{\alpha} f\right)(x) .
$$

Then, from Lemma 3.1 in Pipiras and Taqqu [2000], we have that

$$
(t-s)_{+}^{H-\frac{1}{2}}-(-s)_{+}^{H-\frac{1}{2}}=\Gamma\left(H+\frac{1}{2}\right)\left(D_{-}^{-\left(H-\frac{1}{2}\right)} \mathbf{1}_{(0, t)}\right)(s)
$$




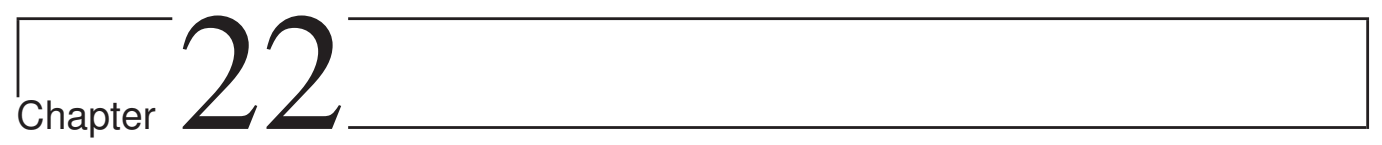

\section{Fractional Brownian Motion}

A fractional Brownian motion ( $\mathrm{fBm}$ ) is a generalization of Brownian motion without independent increments. It represents a natural one-parameter extension and has been widely used to model a number of phenomena in diverse fields from biology to finance. This huge range of potential applications makes fBm an interesting object of study.

This chapter is based on Baghery et al. [2008], Bender [2003] and Chapter 5 in Nualart [2006] .

\section{$22.1 \quad$ Basics}

Definition 22.1 (Fractional Brownian Motion $(\mathrm{fBm})$ ). Let $H \in(0,1)$ be given. A fBm, $\left\{B^{H}(t)\right\}_{t \geq 0}$ of Hurst index $H$ is a continuous and centred Gaussian process with covariance function

$$
R_{H}(t, s):=E\left[B^{H}(t) B^{H}(s)\right]=\frac{1}{2}\left(t^{2 H}+s^{2 H}-|t-s|^{2 H}\right) .
$$

It follows from the definition that the $\mathrm{fBm}$ has the following important properties:

1. $B^{H}(0)=0$,

2. $E\left[B^{H}(t)\right]=0$, for all $t \geq 0$,

3. $B^{H}(t)$ has homogeneous increments,

4. $B^{H}(t)$ is a Gaussian process and $E\left[\left(B^{H}(t)\right)^{2}\right]=t^{2 H}$ for $t \geq 0$,

5. $B^{H}$ has continuous trajectories.

Denote by $\mathcal{S}(\mathbb{R})$ the Schwartz space of rapidly decreasing smooth functions on $\mathbb{R}$ and let $\Omega=$ $\mathcal{S}^{\prime}(\mathbb{R})$ be the topological dual (the space of tempered distributions). We suppress notation by letting $B(t):=B^{(H)}(t)$. 


\subsection{Self-similarity}

\subsubsection{Hölder and Martingale properties}

Lemma 22.2. Let $H \in(0,1)$ then there exists a continuous function $R$ on $\left[-\frac{1}{4}, \frac{1}{4}\right]^{2}$ and a constant $C_{H}$ such that

$$
|R(x, y)| \leq C_{h} \min (|x|,|y|)^{2}|x|^{-H}|y|^{-H}
$$

and for all $(s, t, u, v) \in \mathbb{R}^{4}$ such that $\max \left(\frac{|u|}{|t-s|}, \frac{|v|}{|t-s|}\right) \leq \frac{1}{4}$ we have

$$
\begin{aligned}
& E\left[\left(B^{H}(s+u)-B^{H}(s)\right)\left(B^{H}(t+v)-B^{H}(t)\right)\right]= \\
& H(1-2 H)\left|\frac{|u|}{|t-s|}, \frac{|v|}{|t-s|}\right|^{1-H}+R\left(\frac{u}{t-s}, \frac{v}{t-s}\right) .
\end{aligned}
$$

Proof. See Lemma 1 in Coutin.

From this it follows easily that

Corollary 22.3. The fBm paths have infinite variation on any interval a.s.

Corollary 22.4. For $\alpha>H$, the fBm paths are nowhere locally Hölder continuous of order $\alpha$ a.s.

When constructing the Itô integral we relied heavily on the martingale property. Unfortunately for us we can not do that for fractional Brownian motion.

Proposition 22.5. The $f B m$ is not a semimartingale for $H \neq \frac{1}{2}$.

Proof. See Proposition 5.1.1 in Nualart [2006] or see Baghery et al. [2008].

\subsection{Fractional Dimension}

Proposition 22.6. With probability 1 , the Hausdorff and box dimensions of the graph $\left(t, B^{H}(t)\right)_{t \in[0,1]}$ is equal to $2-H$.

Proof. See Proposition 6 in Coutin.

\subsection{Moving Average Representation}

The following representation of a fBm is due to Mandelbrot and Ness [1968].

Proposition 22.7. Let $\{W(A)\}_{Q \in \mathcal{B}(\mathbb{R}), \mu(A)<\infty}$ be a white noise on $\mathbb{R}$. Then

$$
B_{t}=\frac{1}{C_{1}(H)} \int_{\mathbb{R}}\left[\left((t-s)^{+}\right)^{H-\frac{1}{2}}-\left((-s)^{+}\right)^{H-\frac{1}{2}}\right] d W_{s}
$$


is a $\mathrm{fBm}$ with Hurst parameter $H$, where

$$
C_{1}(H)=\left[\int_{0}^{\infty}\left((1+s)^{H-\frac{1}{2}}-s^{H-\frac{1}{2}}\right)^{2} d s+\frac{1}{2 H}\right]^{\frac{1}{2}} .
$$

\subsection{Representation using Calculus of Variations}

Let $T>0$. Consider a $\operatorname{fBm}\left\{B_{t}\right\}_{t \in[0, T]}$ with $H \in(0,1)$. Let $\mathcal{E}$ be the step functions on $[0, T]$. Let $\mathcal{H}$ be the closure of $\mathcal{E}$ w.r.t. the scalar product

$$
\left\langle\mathbf{1}_{[0, t]}, \mathbf{1}_{[0, s]}\right\rangle_{\mathcal{H}}=R_{H}(t, s) .
$$

By usual arguments we expand the mapping $\mathbf{1}_{[0, T]} \rightarrow B_{t}$ to an isometry between $\mathcal{H}$ and the Gaussian subspace $\mathcal{H}_{1}$. So $\{B(\phi)\}_{\phi \in \mathcal{H}}$ is isonormal Gaussian process as in Definition 19.1.

Let us start by considering: $H>\frac{1}{2}$.

Define a linear operator $K_{H}^{*}$ from $\mathcal{E}$ to $L^{2}([0, T])$ by

$$
\left(K_{H}^{*} \phi\right)(s):=\int_{s}^{T} \phi(t) \frac{\partial K_{H}}{\partial t}(t, s) d t
$$

where

$$
K_{H}(t, s):=c_{H} s^{\frac{1}{2}-H} \int_{s}^{t}(u-s)^{H-\frac{3}{2}} u^{H-\frac{1}{2}} d u,
$$

and $c_{H}=\left[\frac{H(2 H-1)}{\beta\left(2-2 H, H-\frac{1}{2}\right)}\right]^{\frac{1}{2}}$. Then it can be shown that

$$
\left\langle K_{H}^{*} \mathbf{1}_{[0, t]}, K_{H}^{*} \mathbf{1}_{[0, s]}\right\rangle_{L^{2}([0, T])}=\left\langle\mathbf{1}_{[0, t]}, \mathbf{1}_{[0, s]}\right\rangle_{\mathcal{H}} .
$$

It is possible to express the operator $K_{H}^{*}$ in terms of fractional integrals:

$$
\left(K_{H}^{*} \phi\right)(s)=c_{H} \Gamma\left(H-\frac{1}{2}\right) s^{\frac{1}{2}-H}\left(I_{T^{-}}^{H-\frac{1}{2}} u^{H-\frac{1}{2}} \phi(u)\right)(s) .
$$

Now, the process $W=\left\{W_{t}\right\}_{t \in[0, T]}$ defined by

$$
W_{t}=B\left(\left(K_{H}^{*}\right)^{-1}\left(\mathbf{1}_{[0, t]}\right)\right)
$$

is a Wiener process and $\mathrm{B}$ has the integral expression

$$
B_{t}=\int_{0}^{t} K_{H}(t, s) d W_{s}
$$


Now consider the case: $H<\frac{1}{2}$.

Define a linear operator $K_{H}^{*}$ from $\mathcal{E}$ to $L^{2}([0, T])$ by

$$
\left(K_{H}^{*} \phi\right)(s):=K_{H}(T, s) \phi(s)+\int_{s}^{T}\left(\phi(t)-\phi(s) \frac{\partial K_{H}}{\partial r}(t, s) d t,\right.
$$

where

$$
K_{H}(t, s):=c_{H}\left[\left(\frac{t}{s}\right)^{H-\frac{1}{2}}(t-s)^{H-\frac{1}{2}}-\left(H-\frac{1}{2}\right) s^{H-\frac{1}{2}} \int_{s}^{t} u^{H-\frac{3}{2}}(u-s)^{H-\frac{1}{2}} d u,\right.
$$

and $c_{H}=\sqrt{\frac{2 H}{(1-2 H) \beta\left(1-2 H, H+\frac{1}{2}\right)}}$. Now, the process $W=\left\{W_{t}\right\}_{t \in[0, T]}$ defined by

$$
W_{t}=B\left(\left(K_{H}^{*}\right)^{-1}\left(\mathbf{1}_{[0, t]}\right)\right)
$$

is a Wiener process and B has the integral expression

$$
B_{t}=\int_{0}^{t} K_{H}(t, s) d W_{s}
$$

\subsection{Representation using White Noise}

Define for $0<H<1$.

$$
M_{ \pm}^{h} f:= \begin{cases}K_{H} D_{ \pm}^{-\left(H-\frac{1}{2}\right)} f ; & 0<H<\frac{1}{2} \\ f ; & H=\frac{1}{2} \\ K_{H} I_{ \pm}^{H-\frac{1}{2}} f ; & \frac{1}{2}<H<1\end{cases}
$$

From 21.1 we see that a continuous version of $\left\langle\cdot, M_{ \pm}^{h} \mathbf{1}_{(0, t)}\right\rangle$ is a $\mathrm{fBm}$.

\subsection{Integrals w.r.t. fBm}

There are many ways of defining the integral with respect to a fractional Brownian motion. We will explore a few of those.

\subsubsection{Integrals w.r.t. fBm using White Noise}

In this section we define the integral w.r.t. fBm using white noise theory. The first construction uses the standard white noise space. In the second we construct a different white noise space. 


\subsubsection{Wick Itō Skorohod (WIS) Integral for fBm}

In this section we are going to define the integral w.r.t. $\mathrm{fBm}$ for all $H \in(0,1)$. Let $\mathbb{P}$ be the white noise probability measure such that

$$
\int_{\Omega} e^{i\langle\omega, f\rangle} d \mathbb{P}^{H}(\omega)=e^{-\frac{1}{2}\|f\|_{L^{2}(\mathbb{R})}^{2}}
$$

for all $f \in \mathcal{S}(\mathbb{R})$, where $\langle\cdot, \cdot\rangle$ is the dual pairing between $\mathcal{S}(\mathbb{R})$ and $\mathcal{S}^{\prime}(\mathbb{R})$. We are going to relate the $\mathrm{fBm}$ to the standard $\mathrm{Bm}\left(H=\frac{1}{2}\right)$ and to be able to do that we need a special operator.

Definition 22.8 (The M Operator). The operator $M$ is defined on function $f \in \mathcal{S}(\mathbb{R})$ by

$$
\hat{M} f(y)=|y|^{\frac{1}{2}-H} \hat{f}(y), y \in \mathbb{R}
$$

where $\hat{f}(y)$ is the (weighted) Fourier transform.

As usual we extend the operator $M$ to the space

$$
L_{H}^{2}(\mathbb{R}):=\left\{f: \mathbb{R} \rightarrow \mathbb{R},\|f\|_{H}<\infty\right\},
$$

where

$$
\|f\|_{H}:=\|M f\|_{L^{2}(\mathbb{R})}
$$

From the definition 22.8 we see using Parseval's Theorem that

$$
\int_{\mathbb{R}}[M[a, b](x)]^{2} d x=(b-a)^{2 H} .
$$

Using linearity and the polar identity we obtain

$$
\int_{\mathbb{R}} M[0, t] M[0, s] d x=\frac{1}{2}\left(t^{2 H}+s^{2 H}-|t-s|^{2 H}\right) .
$$

We are now ready to define the $\mathrm{fBm}, B^{(H)}(t)$, as the continuous version of

$$
\hat{B}^{(H)}(t):=\hat{B}^{(H)}(\omega, t):=\langle\omega, M[0, t](\cdot)\rangle
$$

It is easy to see that

$$
B^{(H)}(t, \omega)=\sum_{k=1}^{\infty} \int_{0}^{t} M \xi_{k}(s) d s \mathcal{H}_{\epsilon^{(k)}}(\omega)
$$

So we now define the fractional white noise $W^{(H)}(t)$ as

$$
W^{(H)}(t)=\sum_{k=1}^{\infty} M \xi_{k}(t) \mathcal{H}_{\epsilon^{(k)}}(\omega)
$$


so that

$$
\frac{d B^{(H)}(t)}{d t}=W^{(H)}(t)
$$

in $(\mathcal{S})^{*}$.

Definition 22.9 (Definition 4.4.4 in Øksendal and Sulem [2000]). let $Y: \mathcal{R} \rightarrow(\mathcal{S})^{*}$ be such that $Y(t) \diamond W^{(H)}(t)$ is dt-integrable in $(\mathcal{S})^{*}$. Then we say that $Y$ is Wick-Ito-Skorohod (WIS) integrable and we define the integral of $Y$ w.r.t. $B^{(H)}$ by

$$
\int_{\mathbb{R}} Y(t) d B^{(H)}(t):=\int_{\mathbb{R}} Y(t) \diamond W^{(H)}(t) d t
$$

\subsubsection{Fractional Wick Itō Skorohod (fWIS) integrals for fBm with Hurst index}

$$
H>\frac{1}{2}
$$

Let $B_{t}^{H}, t \geq 0$, be a fractional Brownian motion with Hurst index $H \in\left(\frac{1}{2}, 1\right)$ on the probability space $\left(\Omega, \mathcal{F}^{H}, \mathbf{P}^{H}\right)$. We assume $\left(\Omega, \mathcal{F}^{H}, \mathbf{P}^{H}\right)$ is endowed with the natural filtration $\mathcal{F}_{t}^{H}$ of $B^{H}$, where $\mathcal{F}^{H}=\vee_{t \geq 0} \mathcal{F}_{t}^{H}$.

In the following we aim at introducing stochastic integrals with respect to $B_{t}^{H}$ by using techniques from Gaussian white noise analysis. Let $1>H>\frac{1}{2}$, and

$$
\phi(s, t)=\phi_{H}(s, t):=H(2 H-1)|s-t|^{2 H-2} .
$$

For $f, g \in \mathcal{S}(\mathbb{R})$, define

$$
\langle f, g\rangle_{H, t}:=\int_{0}^{t} \int_{0}^{t} f(s) g(r) \phi(s, r) d s d r
$$

and

$$
\langle f, g\rangle_{H}:=\int_{\mathbb{R}} \int_{\mathbb{R}} f(s) g(t) \phi(s, t) d s d t
$$

Now consider the completion of $\mathcal{S}(\mathbb{R})$ under this inner product and denote the resulting separable Hilbert space by

$$
L_{\phi}^{2}
$$

By Bochner-Minlos theorem or an abstract Hilbert space argument we have that there exists a probability measure $\mathbf{P}^{H}$ on the Borel $\sigma$-algebra, $\mathcal{B}(\Omega)$, such that

$$
\int_{\Omega} e^{i\langle\omega, f\rangle} d \mathbf{P}^{H}(\omega)=e^{-\frac{1}{2}\|f\|_{H}^{2}}
$$

for all $f \in \mathcal{S}(\mathbb{R})$, where $\langle\cdot, \cdot\rangle$ is the dual pairing between $\mathcal{S}(\mathbb{R})$ and $\mathcal{S}^{\prime}(\mathbb{R})$. It is now easy to see 
from a Fourier argument that

$$
E_{\mathbf{P}^{H}}[\langle\cdot, f\rangle]:=E[\langle\cdot, f\rangle]=0,
$$

and

$$
E\left[\langle\cdot, f\rangle^{2}\right]=\|f\|_{H}^{2}
$$

Now we define the fractional Brownian motion as

$$
B_{t}^{H}=B^{H}(t, \omega)=\left\langle\omega, \mathbf{1}_{[0, t]}(\cdot)\right\rangle,
$$

by extension, which is an element of $L^{2}\left(\mathbf{P}^{H}\right)$ for each $t$. Let $\mathcal{J}=\left(\mathbb{N}_{0}^{\mathbb{N}}\right)_{c}$ be the set of all finite sequences $\alpha=\left(\alpha_{1}, \ldots, \alpha_{m}\right)$ of nonnegative integers. For $\alpha=\left(\alpha_{1}, \ldots, \alpha_{m}\right)$, let

$$
\mathcal{H}_{\alpha}(\omega):=h_{\alpha_{1}}\left(\left\langle\omega, e_{1}\right\rangle\right) \cdots h_{\alpha_{m}}\left(\left\langle\omega, e_{m}\right\rangle\right)
$$

where $h_{n}$ is the n-th Hermite polynomial (see Holden et al. [2010]) and $\left\{e_{n}\right\}_{n \geq 0}$ a orthonormal basis of $L_{\phi}^{2}(\mathbb{R})$. As in the case of Brownian motion we obtain a Wiener-It $\bar{o}$ chaos expansion.

Theorem 22.10. let $F \in L^{2}\left(\mathbf{P}^{H}\right)$. Then there exist unique $C_{\alpha}, \alpha \in \mathcal{J}$ such that

$$
F(\omega)=\sum_{\alpha \in \mathcal{J}} c_{\alpha} \mathcal{H}_{\alpha}(\omega)
$$

where the convergence is in $F \in L^{2}\left(\mathbf{P}^{H}\right)$. We also have that

$$
\|F\|_{H}^{2}=\sum_{\alpha \in \mathcal{J}} \alpha ! c_{\alpha}^{2}
$$

where $\alpha !:=\alpha_{1} \cdots \alpha_{m}$.

See Holden et al. [2010], Theorem 3.1.8 for a proof. We are now ready to define the fractional Hida test function and distribution spaces.

Definition 22.11 (The Fractional Hida Test Function Space). Let $(\mathcal{S})_{H}$ be the set of all $\psi(\omega)=$ $\sum_{\alpha \in \mathcal{J}} a_{\alpha} \mathcal{H}_{\alpha}(\omega) \in L^{2}\left(\mathbf{P}^{H}\right)$ such that

$$
\|\psi\|_{H, k}^{2}=\sum_{\alpha \in \mathcal{J}} \alpha ! a_{\alpha}^{2}(2 \mathbb{N})^{k \alpha}
$$

where

$$
(2 \mathbb{N})^{\alpha}=\prod_{j}(2 j)^{\gamma_{j}}
$$

Definition 22.12 (The Fractional Hida Distribution Space). Let $(\mathcal{S})_{H}^{*}$ be the set of all formal 
expansions

$$
G(\omega)=\sum_{\beta \in \mathcal{J}} b_{\beta} \mathcal{H}_{\beta}(\omega) \in L^{2}\left(\mathbf{P}^{H}\right)
$$

such that

$$
\|G\|_{H,-q}^{2}=\sum_{\beta \in \mathcal{J}} \beta ! b_{\alpha}^{2}(2 \mathbb{N})^{-q \beta}
$$

for some $q \in \mathbb{N}$.

Let $(\mathcal{S})_{H}$ be equipped with the projective topology and $(\mathcal{S})_{H}^{*}$ with the inductive topology. Then $(\mathcal{S})_{H}^{*}$ is isomorph (in the category of topological vector spaces) to the topological dual of $(\mathcal{S})_{H}$ with action given by

$$
\ll G, \psi \gg:=\langle G, \psi\rangle_{(\mathcal{S})_{H}}:=\sum_{\alpha \in \mathcal{J}} \alpha ! a_{\alpha} b_{\alpha} .
$$

Definition 22.13. Let

$$
F(\omega)=\sum_{\alpha \in \mathcal{J}} a_{\alpha} \mathcal{H}_{\alpha}(\omega)
$$

and

$$
G(\omega)=\sum_{\beta \in \mathcal{J}} b_{\beta} \mathcal{H}_{\beta}(\omega)
$$

belong to $(\mathcal{S})_{H}^{*}$. Then we define the Wick product $F \diamond G$ by

$$
F \diamond G(\omega)=\sum_{\alpha, \beta \in \mathcal{J}} a_{\alpha} b_{\beta} \mathcal{H}_{\alpha+\beta}(\omega)
$$

Further, let

$$
D_{t}^{\phi} F=\int_{\mathbb{R}} D_{s}^{H} F \phi(t, s) d s
$$

denote the Malliavin $\phi$-derivative of F (see Øksendal and Sulem [2000] Definition 3.5.1).

\subsubsection{Integrals w.r.t. fBm using Youngs Integral}

Proposition 22.14 (Young [1936]). Let $p, q$ in $[1, \infty)$ such that $\frac{1}{p}+\frac{1}{q}>1$. Assume that $x$ has finite p-variation and y finite q-variation. The following sequence of riemann sums converges:

$$
\sum_{i=1}^{k_{n}-1} x\left(t_{i}^{n}\right)\left[y\left(t_{i+1}^{n}\right)-y\left(t_{i}^{n}\right)\right]
$$


where $\pi^{n}=\left(t_{i}^{n}\right)_{i=1}^{k_{n}}$ is any sequence of finite subdivisions of $[0,1]$ with mesh going to 0 . The limit denoted by $\int_{0}^{t} x(s) d y(s)$, does not depend upon the choice of the sequence of subdivisions.

We then have the following change of variable formula:

Corollary 22.15. let $x$ be a path of finite p-variation with $1 \leq p<2$. Let $\left\{f_{i}\right\}_{i=0}^{d}$ be a differentiable vector field on $\mathbb{R}^{n}, \alpha$-Hölder continuous for some $\alpha>p-1$. Then

$$
f(x(1))=f(x(0))+\sum_{i=1}^{d} \int_{0}^{t} \frac{\partial f}{\partial x^{i}}(x(s)) d x^{i}(s) .
$$

For $H>\frac{1}{2}$ it was in Zähle [1998] shown that almost surely, the sample paths of $W^{H}$ belongs to the Besov space $\mathcal{W}_{2, \infty}^{\beta}$ for $\frac{1}{2}<\beta<H$. Here $\mathcal{W}_{2, \infty}^{\beta}$ is the Besov space of all bounded measurable functions $f:[0,1] \rightarrow \mathbb{R}$ such that

$$
\int_{0}^{1} \int_{0}^{1} \frac{(f(t)-f(s))^{2}}{|-s|^{2 \beta+1}} d s d t<\infty .
$$

\subsubsection{Integrals w.r.t. fBm using Rough Paths}

Theorem 22.16. Let $W=\left(w_{t}^{i}, t \geq 0, i=1, \ldots, d\right)$ be a d-dimensional fractional Brownian motion with Hurst parameter $H$ and let $\boldsymbol{W}(m)_{s, t}=\left(1, \boldsymbol{W}(m)_{s, t}^{1}, \boldsymbol{W}(m)_{s, t}^{2}, \boldsymbol{W}(m)_{s, t}^{3}\right)$ be the smooth rough path associated with the m-th dyadic approximation of $W$. If $H \in\left(\frac{1}{4}, \frac{1}{2}\right)$ then for any $p<4$ such that $H p>1, \boldsymbol{W}(m)$ converges almost surely to a geometric rough path $\boldsymbol{W}_{s, t}=\left(1, \boldsymbol{W}_{s, t}^{1}, \boldsymbol{W}_{s, t}^{2}, \boldsymbol{W}_{s, t}^{3}\right)$ in p-variation distance. Moreover $\boldsymbol{W}_{s, t}^{1}=W_{t}-W_{s}$. However, if $H \leq \frac{1}{4}$, then even the second level paths $\boldsymbol{W}(m)^{2}$ of its dyadic approximations do not converge in $L^{1}(\Omega, \mathcal{F}, \mathbf{P})$ according to the p-variation distance.

Proof. See theorem 2 in Coutin and Qian [2002]. 


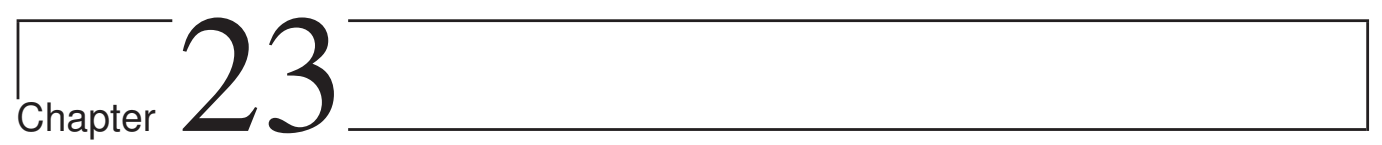

\section{Banach Valued Processes}

By addition of a small amount of operator theory the classical Itô integral can be easily generalized to integrands with values in Hilbert spaces and Hilbert space valued Wiener processes. Recently, van Neerven and Weis introduced for deterministic Banach space valued integrands a stochastic integral with respect to Wiener processes on Banach spaces without any conditions on the underlying Banach space. With Veraar they were able to extend this theory to random integrands by requiring the Banach space to be a UMD space.

This is based on Riedle [2009a], van Nerven [2008] and Dalang and Quer-Sardanyans [2010].

\subsection{Spatially Homogeneous Noise and Random Fields}

We start with a Hilbert space $V$ and a probability space $(\Omega, \mathcal{F}, \mathcal{P})$, satisfying the usual conditions, consider a family of zero mean gaussian variables $\{W(\phi)\}_{\phi \in C_{0}^{\infty}\left(\mathbb{R}^{d+1}\right)}$, where $\mathbb{R}^{d+1}$ denotes the space of infinitely differentiable functions with compact support, with covariance

$$
E[W(\phi) W(\psi)]=\int_{0}^{\infty} \int_{\mathbb{R}^{d}}(\phi(t) * \tilde{\psi}(t))(x) \mathcal{T}(d x) d t
$$

where $*$ denotes convolution in the spatial variable, $\tilde{\psi}(t, x):=\psi(t,-x)$ and $\mathcal{T}$ is a non-negative definite tempered measure on $\mathcal{R}^{d}$. Note that for all $\phi$ belonging to the space of $\mathcal{S}\left(\mathbb{R}^{d}\right)$ of rapidly decreasing $C^{\infty}$ functions,

$$
\int_{\mathbb{R}^{d}} \phi(x) \mathcal{T}(d x)=\int_{\mathbb{R}^{d}} \mathcal{F} \phi(\xi) \mu(d \xi),
$$

and there exists an integer $m \geq 1$ such that

$$
\int_{\mathbb{R}^{d}}\left(1+|\xi|^{2}\right)^{-m} \mu(d \xi)<\infty
$$

Where $\mathcal{F} \phi$ is the Fourier transform of $\phi \in \mathcal{S}\left(\mathbb{R}^{d}\right)$

$$
\mathcal{F} \phi(\xi)=\int_{\mathbb{R}^{d}} \phi(x) e^{-2 \pi i \xi \times x} d x .
$$


See Rudin [1991] or Chapter 20 for more information. We associate a Hilbert space $U$ with $W$ the following way:

Let $U$ be the completion of the Schwartz space $\mathcal{S}\left(\mathbb{R}^{d}\right)$ endowed with the semi-inner product

$$
\langle\phi, \psi\rangle_{U}=\int_{\mathbb{R}^{d}}(\phi(t) * \tilde{\psi}(t))(x) \mathcal{T}(d x)=\int_{\mathbb{R}^{d}} \mathcal{F} \phi(\xi) \mathcal{F} \bar{\psi}(\xi) \mu(d \xi),
$$

for $\phi, \psi \in \mathcal{S}\left(\mathbb{R}^{d}\right)$ and associated semi-norm $\|\cdot\|_{u}$.

Lemma 23.1. The space $C_{0}^{\infty}\left([0, T] \times \mathcal{R}^{d}\right)$ is dense in $U_{T}=L^{2}([0, T], U)$ for $\|\cdot\|_{U_{t}}$.

Proof. See Lemma 2.4 in Dalang and Quer-Sardanyans [2010].

The covariance (23.1) shows that the generalized Gaussian random field $\{W(\phi)\}_{\phi \in C_{0}^{\infty}\left([0, T] \times \mathbb{R}^{d+1}\right)}$ is a random linear functional, i.e.

$$
W(a \phi+b \psi)=a W(\phi)+b W(\psi),
$$

and $\phi \mapsto W(\phi)$ is an isometry from $\left(C_{0}^{\infty}\left([0, T] \times \mathbb{R}^{d+1}\right),\|\cdot\|_{U_{t}}\right)$ into $L^{2}(\Omega, \mathcal{F}, \mathcal{P})$. Using the above Lemma (23.1) we get the following proposition.

Proposition 23.2. For $t \geq 0$ and $\phi \in U$ let $W_{t}(\phi)=W\left(\mathbf{1}_{[0 ; T]}(\cdot) \phi(\star)\right)$ Then $\{W(\phi)\}_{\phi \in U}$ is a cylindrical Wiener process with $Q=I d_{U}$.

\subsubsection{Walsh Method}

Walshs theory of integration uses the concept explored in Section 9.3.1. Let $\left\{M_{t}(A), \mathcal{F}_{t}, t \in\right.$ $\left.[0, T], A \in \mathcal{B}_{b}\left(\mathbb{R}^{d}\right)\right\}$ be a Martingale measure. This Martingale measure is constructed by approximation, extending the $W$ to indicator functions and then letting

$$
M_{t}(A):=W\left(\mathbf{1}_{[0 ; T]}(\cdot) \phi(\star)\right) .
$$

The Walsh integral, $g \dot{M}$ is defined for $g \in P_{+}$where $P_{+}$is the set of predictable processes $(\omega, t, x) \mapsto g(\omega, t, x)$ such that

$$
\|g\|_{+}^{2}:=E\left[\int_{0}^{T} \int_{\mathbb{R}^{d}}(|g(t, \star) * \tilde{g}(t, \star)|)(x) \mathcal{T}(d x)\right]<\infty
$$

For $g \in P_{+}$we let

$$
\|g\|_{0}^{2}:=E\left[\|g\|_{U_{T}}^{2}\right]=E\left[\int_{0}^{T} \int_{\mathbb{R}^{d}}(g(t, \star) * \tilde{g}(t, \star))(x) \mathcal{T}(d x)\right] .
$$

Let $g \dot{M}$ denote the Walsh integral.

$$
g \dot{W}=\int_{0}^{t} g_{s} d W_{s}:=\int_{j=1}^{\infty} \int_{0}^{T}\left\langle g_{s}, e_{j}\right\rangle_{u} d W_{s}\left(e_{j}\right) .
$$




\subsubsection{Connection between the two integrals}

\section{Proposition 23.3.}

1. If $g \in P_{+}$, then $g \in L^{2}(\Omega \times[0, T], U)$ and $g \cdot M=g \cdot W$, where the left-hand side is a Walsh integral and the right-hand is defined by (23.2).

2. If $g \in P_{0}$, then $g \in L^{2}(\Omega \times[0, T], U)$ and $g \cdot M=g \cdot W$, where the left-hand side is a Walsh integral and the right-hand is defined by (23.2).

Proof. See Proposition 2.6 in Dalang and Quer-Sardanyans [2010].

\subsection{Banach Spaces}

Let $U$ be a separable Banach Space over $\mathbb{R}$ with norm $\|\cdot\|_{U}$ (we use $\|\cdot\|$ if there are no confusion can arise). Let $U^{*}$ denote the (topological) dual space. Let $(\Omega, \mathcal{F}, \mathbf{P})$ be a probability space with filtration $\{\mathcal{F}\}_{t \geq 0}$.

Lemma 23.4. Let $T: U^{*} \rightarrow \mathbb{R}$ be a linear functional. Then the following is equivalent:

1. There exists $u_{t} \in U$ such that $T u^{*}=\left\langle u_{t}, u^{*}\right\rangle$ for all $u^{*} \in U$.

2. $T$ is weakly* continuous.

3. $T$ is weakly* sequentially continuous.

Proof. See Megginson [1998].

Remember from the Hahn-Banach Theorem that

$$
\|u\|_{U}=\sup _{\left\|u^{*}\right\|}\left|\left\langle u, u^{*}\right\rangle\right|
$$

Definition 23.5. A linear subspace $V$ of $U^{*}$ is called norming for a subset $S$ of $U$ if

$$
\|u\|=\sup \left\{\mid\left\langle u, u^{*}\right\rangle: u^{*} \in V,\left\|u^{*}\right\| \leq 1\right\} \text { for all } u \in S .
$$

Let $\Gamma \subseteq U^{*}$. The set of the form

$$
Z\left(u_{1}^{*}, \ldots, u_{n}^{*} ; F\right):=\left\{u \in U:\left(\left\langle u, u_{1}^{*}\right\rangle, \ldots,\left\langle u, u_{n}^{*}\right\rangle\right) \in F\right\}
$$

where $u_{1}^{*}, \ldots, u_{n}^{*} \in \Gamma$ and $F \in \mathcal{B}(\mathbb{R})$ are called cylindrical sets. The set of all cylindrical sets

$$
Z\left(u_{1}^{*}, \ldots, u_{n}^{*} ; F\right):=\left\{u \in U:\left(\left\langle u, u_{1}^{*}\right\rangle, \ldots,\left\langle u, u_{n}^{*}\right\rangle\right) \in F\right\}, u_{1}^{*}, \ldots, u_{n}^{*} \in \Gamma, F \in \mathcal{B}(\mathbb{R}), n \in \mathbb{N}
$$

is denoted by $\mathcal{L}(U, \Gamma)$ and is an algebra. The corresponding $\sigma$-algebra is denoted by $\mathcal{C}(U, \Gamma)$. For $\Gamma=U^{*}$ we write $\mathcal{L}(U):=\mathcal{L}(U, \Gamma)$.

Definition 23.6. A measurable mapping $X: \Omega \rightarrow U$ is called an $U$-valued random variable. 
Definition 23.7. A mapping $X: \Omega \rightarrow U$ is called weakly measurable if

$$
\omega \mapsto\left\langle X(\omega), u^{*}\right\rangle
$$

is $\mathcal{F} / \mathcal{B}\left(\mathbb{R}\right.$-measurable for all $u^{*} \in U^{*}$.

Definition 23.8. The characteristic function $\phi_{\mu}: U^{*} \rightarrow \mathbb{C}$ of a probability measure $\mu$ on $\mathcal{B}(U)$ is defined by

$$
\phi_{\mu}\left(u^{*}\right)=\int_{U} e^{i\left\langle u, u^{*}\right\rangle} \mu(d u)
$$

If $X$ is a random variable denote by $\phi_{X}$ the characteristic function of $\mathbf{P}_{X}$.

Theorem 23.9. If $U$ is separable then given two probability measures $\mu_{1}$ and $\mu_{2}$ on $\mathcal{B}(U)$, the following is equivalent:

1. $\mu_{1}=\mu_{2}$.

2. $\phi_{\mu_{1}}=\phi_{\mu_{2}}$.

Theorem 23.10 (Bochner). For a function $\phi: \mathbb{R}^{n} \rightarrow \mathbb{C}$ the following is equivalent:

1. $\phi$ is the characteristic function of a probability measure on $\mathcal{B}(U)$.

2. $\phi$ is positive-definite, continuous and $\phi(0)=1$.

\subsubsection{Gaussian Measures}

We call a measure $\mu$ on $\mathcal{B}(U)$ a Gaussian measure with mean $m \in \mathbb{R}$ and variance $\sigma^{2} \geq 0$ if either $\mu=\delta_{m}$ and $\sigma^{2}=0$ or it has the density

$$
f(s)=\frac{1}{\sqrt{2 \pi \sigma^{2}}} \exp \left(-\frac{1}{2 \sigma^{2}}(s-m)^{2}\right) .
$$

Definition 23.11. A probability measure $\mu$ on $\mathcal{B}(U)$ is called Gaussian if

$$
\mu \circ\left(u^{*}\right)^{-1}: \mathcal{B}(\mathbb{R}) \rightarrow[0,1]
$$

is a Gaussian measure for all $u^{*} \in U^{*}$. A random variable $i$ called Gaussian if $P_{X}$ is Gaussian.

Definition 23.12. Let $\mu$ be a Gaussian measure on $\mathcal{B}(U)$. The element $m \in U$ such that

$$
\left\langle m, u^{*}\right\rangle=\int_{U}\left\langle u, u^{*}\right\rangle \mu(d u)
$$

for all $u^{*} \in U^{*}$, is called the mean of $\mu$. The operator $Q \in \mathcal{L}\left(U^{*}, U\right.$

$$
\left\langle Q u^{*}, v^{*}\right\rangle=\int_{U}\left\langle u, u^{*}\right\rangle\left\langle v, v^{*}\right\rangle \mu(d u)-\left\langle m, u^{*}\right\rangle\left\langle m, v^{*}\right\rangle
$$

for all $u^{*}, v^{*} \in U^{*}$, is called the covariance operator of $\mu$. 
Theorem 23.13. For a measure $\mu$ on $\mathcal{B}(U)$ the following is equivalent:

1. $\mu$ is Gaussian.

2. the characteristic function $\phi_{\mu}$ is on the form

$$
\phi_{\mu}=e^{i\left\langle m, u^{*}\right\rangle-\frac{1}{2}\left\langle Q u^{*}, u^{*}\right\rangle},
$$

for some $m \in U$ and some positive symmetric operator $Q \in \mathcal{L}\left(U^{*}, U\right)$.

Proof. See Theorem 1.1.32 in Riedle [2009a].

Theorem 23.14 (Sazonov). Let $U=H$ be a Hilbert space. For an operator $Q \in \mathcal{L}\left(U^{*}, U\right)$ the following is equivalent:

1. $Q$ is the covariance operator of a Gaussian measure on $\mathcal{B}(H)$.

2. $Q$ is positive, symmetric and nuclear.

\subsubsection{Reproducing Kernel Hilbert Space}

Every positive, symmetric operator $Q: U^{*} \rightarrow U$ can be factorized through a Hilbert space. On the range of $Q$ define a bilinear form

$$
\left[Q u^{*}, Q v^{*}\right]_{H_{Q}}:=\left\langle Q u^{*}, v^{*}\right\rangle \text {. }
$$

Denote the completion of the range of $Q$ with respect to $[\cdot, \cdot]_{H_{Q}}$ by $H_{Q}$.

We list some properties of $Q$ and $H_{Q}$ :

1. The inclusion mapping is continuous on the range of $Q$ and it extends to a bounded linear operator $i_{Q}$ from $H_{Q}$ to $U$.

2. The operator $Q$ decomposes as

$$
Q=i_{q} i_{Q}^{*}
$$

3. The range of $i_{Q}^{*}$ is dense in $H_{Q}$.

4. The inclusion mapping $i_{Q}$ is injective.

5. If $U$ is separable so is $H_{Q}$.

\subsection{Cylindrical Versions}

\subsubsection{Cylindrical Measures}

Definition 23.15. A function

$$
\mu: \mathcal{L}(U) \rightarrow[0, \infty]
$$

is called a cylindrical measure on $\mathcal{L}(U)$, if for each finite subset $\Gamma \subseteq U^{*}$ the restriction of $\mu$ to the $\sigma$-algebra $\mathrm{C}(U, \Gamma)$ is a measure. 
As we had for measure we have for cylindrical measures the definition of characteristic functions.

Definition 23.16. The characteristic function $\phi_{\mu}: U^{*} \rightarrow \mathbb{C}$ of a cylindrical measure $\mu$ on $\mathcal{L}(U)$ is defined by

$$
\phi_{\mu}\left(u^{*}\right)=\int_{U} e^{i\left\langle u, u^{*}\right\rangle} \mu(d u)
$$

If $X$ is a random variable denote by $\phi_{X}$ the characteristic function of $\mathbf{P}_{X}$.

We have the following.

Theorem 23.17. A function $\phi: U^{*} \rightarrow \mathcal{C}$ is a characteristic function of a cylindrical probability measure on $U$ if and only if

1. $\phi(0)=1$.

2. $\phi$ is positive definite.

3. The restriction of $\phi$ to every finite dimensional subset $\Gamma \subseteq U^{*}$ is continuous with respect to the norm topology.

Proof. See theorem 1.2.5 in Riedle [2009a].

\subsubsection{Cylindrical Random Variables}

Definition 23.18. A cylindrical random variable $Y$ in $U$ is a linear map

$$
Y: u^{*} \rightarrow L^{0}(\Omega, \mathbb{R})
$$

\subsubsection{Radonification}

Definition 23.19. $\quad$ 1. A cylindrical measure $\mu$ on $\mathcal{L}(U)$ extends to a Radon measure on $\mathcal{B}(U)$ if there exist a Radon measure $\nu$ on $\mathcal{B}(U)$ such that

$$
\nu(Z)=\mu(Z)
$$

for all $Z \in \mathcal{L}(U)$.

2. A cylindrical random variable $X$ in $U$ is called induced by an $U$-random variable $Y$ if, a.s.

$$
X u^{*}=\left\langle Y, u^{*}\right\rangle
$$

for all $u^{*} \in U^{*}$.

Definition 23.20. A cylindrical measure $\mu$ on $\mathcal{L}(U)$ is called tight if for each $\epsilon>0$ there exists a compact set $K=K(\epsilon) \subseteq U$ such that

$$
\mu \circ \pi_{u_{1}^{*}, \ldots, u_{n}^{*}}^{-1}\left(\pi_{u_{1}^{*}, \ldots, u_{n}^{*}}(K)\right) \geq 1-\epsilon,
$$

for all $u_{1}^{*}, \ldots, u_{n}^{*}$ and all $n \in \mathbb{N}$ 
Theorem 23.21 (Prokhorov). For a cylindrical measure $\mu$ on $\mathcal{L}(U)$ the following is equivalent:

1. $\mu$ extends to a Radon measure.

2. $\mu$ is tight.

\subsubsection{Gaussian Cylindrical Measures}

Definition 23.22. A cylindrical measure $\mu$ on $\mathcal{L}(U)$ is called weakly Gaussian if

$$
\mu \circ\left(u^{*}\right)^{-1}
$$

is a Gaussian measure on $\mathcal{B}(U)$.

Definition 23.23. Let $\mu$ be a weakly Gaussian cylindrical measure on $\mathcal{L}(U)$. The functional

$$
m\left(u^{*}\right):=\int_{U}\left\langle u, u^{*}\right\rangle \mu(d u)
$$

is called the mean of $\mu$. The operator

$$
\left(r u^{*}\right) v^{*}:=\int_{U}\left\langle u, u^{*}\right\rangle\left\langle v, v^{*}\right\rangle \mu(d u)-m\left(u^{*}\right) m\left(v^{*}\right),
$$

is called the covariance operator of $\mu$.

Definition 23.24. A centred weakly Gaussian cylindrical measure $\mu$ on $\mathcal{L}(U)$ is called strongly Gaussian if the covariance operator $Q: U^{*} \rightarrow\left(U^{*}\right)^{\prime}$ is $U$-valued.

Here $U^{\prime}$ is the algebraic dual of $U$.

\subsection{5 $\gamma$-Operators}

If we want to generalize to Banach spaces from Hilbert spaces some care must be taken. Three steps are often enough to allow us to generalize results from the Hilbert case. These are:

1. Replace functions by $\gamma$-radonifying operators.

2. Replace uniform boundedness by $\gamma$-boundedness.

3. Replace orthogonality by "unconditionality".

Definition 23.25. A linear operator $S: H \rightarrow U$ is called $\gamma$-summing if for some, or equivalently for all, $1 \leq p<\infty$,

$$
\|S\|_{\gamma_{p}^{\infty}(H, U)}:=\sup \left(E\left[\left\|\sum_{j=1}^{k} \gamma_{j} S h_{j}\right\|^{p}\right]\right)^{\frac{1}{p}}<\infty
$$

where the supremum is taken over all finite orthonormal systems $\left\{h_{1}, \ldots, h_{k}\right\}$.

Let $\gamma^{\infty}(H, U)$ denote the linear space of all $\gamma$-summing operators from $H$ to $U$. 
Proposition 23.26. The space $\gamma^{\infty}(H, U)$ is a Banach space.

Proof. See proposition 5.2 in van Nerven [2008].

Definition 23.27. Let $\gamma$ be the standard Gaussian cylindrical measure on a separable Hilbert space $H$. A linear bounded operator $F: H \rightarrow U$ is called $\gamma$-radonifying if the cylindrical measure $\gamma \circ F^{-1}$ extends to a Gaussian measure on $\mathcal{B}(U)$.

For $h \in H$ and $u \in U$ we denote by $h \otimes u$ the operation in $\mathcal{L}(H, U)$ defined by

$$
(h \otimes u) h^{\prime}:=\left[h, h^{\prime}\right] u
$$

for $h^{\prime} \in H$. An operator in $\mathcal{L}(H, U)$ is called an finite rank operator if it is finite linear combinations of the form (23.3).

Definition 23.28. The space $\gamma(H, U)$ is the closure of $\gamma^{\infty}(H, U)$ of all finite rank operators.

Theorem 23.29. Suppose $Q \in \mathcal{L}\left(U^{*}, U\right)$ and $R \in \mathcal{L}(H, U)$ satisfies $Q=R R^{*}$. Then the following is equivalent:

1. $Q$ is a Gaussian covariance operator;

2. $R \in \gamma(H, U)$.

Proof. See Theorem 5.16 in van Nerven [2008].

Theorem 23.30. Let $\gamma$ be the standard Gaussian cylindrical measure on a separable Hilbert space $H$ with orthonormal basis $\left\{e_{k}\right\}_{k \in \mathbb{N}}$ and let $\left\{G_{k}\right\}_{k \in \mathbb{N}}$ be a sequence of independent standard real normal random variables. For $F \in \mathcal{L}(H, U)$ the following is equivalent:

1. $F$ is $\gamma$-radonifying;

2. the operator $F F^{*}: U^{*} \rightarrow U$ is the covariance operator of a Gaussian measure on $\mathcal{B}(U)$;

3. the series $\sum_{k=1}^{\infty} G_{k} F e_{k}$ converges a.s. in $U$.

4. the series $\sum_{k=1}^{\infty} G_{k} F e_{k}$ converges a.s. in $L^{p}(\Omega, U)$ for some $p \in[1, \infty)$.

5. the series $\sum_{k=1}^{\infty} G_{k} F e_{k}$ converges a.s. in $L^{p}(\Omega, U)$ for all $p \in[1, \infty)$.

If anybody or equivalently all of the above is true then we have that for every $p \in[1, \infty)$

$$
\int_{U}\|u\|^{p} \mu(d u)=E\left\|\sum_{k=1}^{\infty} G_{k} F e_{k}\right\|^{p} .
$$

\subsection{Wiener Processes}

Definition 23.31. An adapted process $\left\{W_{t}\right\}_{t \geq 0}$ in $U$ is called a Wiener process if

1. $W(0)=0$ P-a.s.

2. $W$ has independent, stationary increments.

3. There exists a Gaussian covariance operator $Q: U^{*} \rightarrow U$ such that

$$
W(t)-W(s) \stackrel{d}{=} N(0,(t-s) Q)
$$

for all $0 \leq s \leq t$ 


\subsubsection{Cylindrical Wiener Processes}

Definition 23.32. An cylindrical process $\left\{X_{t}\right\}_{t \geq 0}$ in $U$

1. is called adapted to a given filtration $\left\{\mathcal{F}_{t}\right\}_{t \geq 0}$ if $X(t) u^{*}$ is $\mathcal{F}_{t}$-measurable for all $t \geq 0$ and all $u^{*} \in U^{*}$.

2. $X$ has weakly independent increments if for all $0 \leq t_{0} \leq t_{1} \leq \cdots \leq t_{n}$ and all $u_{1}^{*}, ; u_{n}^{*} \in U^{*}$ the random variables

$$
\left(X\left(t_{1}\right)-X\left(t_{0}\right)\right) u_{1}^{*}, \ldots,\left(X\left(t_{n}\right)-X\left(t_{n-1}\right) u_{n}^{*}\right.
$$

are independent.

Definition 23.33. An adapted cylindrical process $\left\{W_{t}\right\}_{t \geq 0}$ in $U$ is a cylindrical Wiener process if

1. for all $n \in \mathbb{N}$ and $u_{1}^{*}, ; u_{n}^{*} \in U^{*}$ the $\mathbb{R}^{n}$-valued stochastic process

$$
\left\{W(t) u_{1}^{*}, \ldots, W(t) u_{n}^{*}\right\}_{t \geq 0}
$$

is a Wiener process.

2. The cylindrical distribution $\mu$ of $W(1)$ is strongly Gaussian.

Theorem 23.34. For an adapted cylindrical process $\left\{W_{t}\right\}_{t \geq 0}$ in $U$ the following is equivalent:

1. W is a cylindrical Wiener process.

2. There exists a Hilbert space $H$ with an orthonormal basis $\left\{e_{k}\right\}_{k \in \mathbb{N}}, F$ inL $(H, U)$ and independent real valued standard Wiener process $\left\{B_{k}\right\}_{k \in \mathbb{N}}$

such that

$$
W(t) U^{*}=\sum_{k=1}^{\infty}\left\langle F e_{k}, u^{*}\right\rangle B_{k}(t),
$$

where the convergence is in $L^{2}(\Omega, \mathbb{R})$ and holds for all $t \geq 0$.

Proof. See Riedle [2009b].

Theorem 23.35. For an adapted cylindrical process $\left\{W_{t}\right\}_{t \geq 0}$ in $U$ the following is equivalent:

1. $W$ is a cylindrical Wiener process.

2. There exists a Hilbert space $H$ with an orthonormal basis $\left\{e_{k}\right\}_{k \in \mathbb{N}}, a \gamma$-radonifying operator $F$ inL $(H, U)$ and independent real valued standard Wiener process $\left\{B_{k}\right\}_{k \in \mathbb{N}}$

such that

$$
W(t) U^{*}=\sum_{k=1}^{\infty} F e_{k} B_{k}(t),
$$

where the convergence is in $L^{2}(\Omega, U)$ and holds for all $t \geq 0$.

Proof. See Riedle [2009a]. 


\subsubsection{Cylindrical Stochastic Integral}

In this section we assume that $W$ ha the representation belonging to Theorem 23.34:

$$
W(t) U^{*}=\sum_{k=1}^{\infty}\left\langle i_{Q} e_{k}, u^{*}\right\rangle B_{k}(t),
$$

where $H$ is the reproducing kernel Hilbert space of the covariance operator $Q$ with inclusion mapping $i_{q}: H \rightarrow U$ and an orthonormal basis $\left\{e_{k}\right\}_{k \in \mathbb{N}}$ of $H$. The real valued standard Wiener process $\left\{B_{k}\right\}_{k \in \mathbb{N}}$ are defined by $B_{k}(t)=W(t) u_{k}^{*}$ for some $u_{k}^{*} \in U^{*}$ with $i_{Q}^{*} u_{k}^{*}=e_{k}$.

Definition 23.36. Let $M_{T}(U, V)$ be the set of all random variables $\Phi:[0 ; T] \times \Omega \rightarrow L(U, V)$ such that

1.

$$
(t, \omega) \mapsto i_{Q}^{*} \Phi^{*}(t, \omega) v^{*}
$$

is $\left(\mathcal{B}([0, T] \otimes \mathcal{A} \mathcal{B}(H))\right.$-measurable for all $v^{*} \in V^{*}$.

2.

$$
\omega \mapsto \Phi^{*}(t, \omega) v^{*}
$$

is $\mathcal{F}_{t}$-measurable for all $v^{*} \in V^{*}$ and all $t \in[0, T]$.

3.

$$
\int_{=} E\left\|i_{Q}^{*} \Phi^{*}(s, \cdot) v^{*}\right\|_{H}^{2} d s<\infty
$$

for all $v^{*} \in V^{*}$.

Definition 23.37. For $\Phi \in M_{T}(U, V)$ let

$$
I_{t}(\Phi) v^{*}:=\sum_{k=1}^{\infty} \int_{0}^{t}<\Phi(s) i_{Q} e_{k}, v^{*}>d B_{k}(s)
$$

in $L^{2}(\Omega, \mathbb{R})$ for all $v^{*} \in V^{*}$ and all $t \in[0, T]$.

It can be shown that the limit exists and are independent of the representation of $W$. See Riedle [2009a] Lemma 2.2.3.

Definition 23.38. For $\Phi \in M_{T}(U, V)$ we call the cylindrical random variable

$$
I_{t}(\Phi):=\int_{0}^{t} \Phi(s) d W(s)
$$

the cylindrical stochastic integral w.r.t. $W$. 


\subsubsection{Stochastic Pettis Integral}

First we consider the case $U=\mathbb{R}$.

\subsubsection{Real Valued Wiener Processes}

Let $M_{t}(\mathbb{R}, V)$ be the set of non-random functions $F:[0, T] \rightarrow V$ which is weakly measurable and

$$
\int_{0}^{T}\left|\left\langle F(s), v^{*}\right\rangle\right|^{2} d s<\infty
$$

for all $v^{*} \in V^{*}$. Then $F$ is called a $V$-weakly $L^{2}([0, T])$ function.

Definition 23.39. A function $F:[0, T] \rightarrow V$ is called stochastically Pettis integrable with respect to $B$ if it is $V$-weakly $L^{2}([0, T])$ and there exists a $V$-valued random variable $Y$ such that for all $v^{*} \in V^{*}$

$$
Z v^{*}:=\left\langle Y, v^{*}\right\rangle=\int_{0}^{T}\left\langle F(t), v^{*}\right\rangle d B(t)
$$

a.s. and we denote

$$
Y=\int_{0}^{T} F(t) d B(t) .
$$

The cylindrical random variable $Z$ is induced by a random variable $Y$ in $V$ if and only if the linear mapping $I_{F}$ is $\gamma$-radonifying.

Lemma 23.40. For a $V$-weakly $L^{2}([0, T])$ function $F:[0, T] \rightarrow V$ we let

$$
\left\langle I_{F} f, v^{*}\right\rangle=\int_{0}^{T}\left\langle F(t), v^{*}\right\rangle f(t) d t
$$

for $f \in L^{2}([0, T])$ and for all $v^{*} \in V^{*}$. $I_{F}$ is a bounded linear operator.

Proof. See Riedle [2009a] Lemma 2.3.2.

Let $\left\{f_{k}\right\}_{k \in \mathbb{N}}$ be an orthonormal basis of $L^{2}([0, T[)$ and

$$
\gamma_{k}:=\int_{0}^{T} f_{k}(t) d B(t)
$$

Then, the random variables $\left\{\gamma_{k}\right\}_{k \in \mathbb{N}}$ are real valued, independent centred Gaussian random variables with $E\left[\gamma_{k}\right]^{2}=\sigma^{2}$.

Denote by $L_{\frac{1}{\sigma}}^{2}$ the space $L^{2}([0, T])$ with the norm $\|h\|_{L_{\frac{1}{\sigma}}^{2}}=|\sigma|\|h\|_{L^{2}}$. 
Lemma 23.41. $\quad 1 . \Gamma$ is a well defined Gaussian cylindrical random variable on $L^{2}([0, T])$ with

$$
\phi_{\Gamma}(f)=\exp \left(i \frac{1}{2}\|f\|_{L_{\frac{1}{\sigma}}^{2}}^{2}\right)
$$

The definition of $\Gamma$ does not depend on $\left\{f_{k}\right\}_{k \in \mathbb{N}}$.

2. $Z$ is a Gaussian cylindrical random variable with characteristic function

$$
\phi_{\Gamma}\left(v^{*}\right)=\exp \left(i \frac{1}{2} \int_{0}^{T}\left\langle F(t), v^{*}\right\rangle^{2} d t\right) .
$$

3. $Z v^{*}=\Gamma I_{F}^{*} v^{*}$ for all $v^{*} \in V^{*}$,

4. if $\mu$ denotes the cylindrical distribution of $\Gamma$, then

$$
\phi_{Z}=\phi_{\mu \circ I_{f}^{-1}}
$$

Theorem 23.42. For a $V$-weakly $L^{2}([0, T])$ function $F:[0, T] \rightarrow V$ the following is equivalent:

1. F is stochastically Pettis integrable with respect to $B$.

2. The cylindrical integral $I_{T}(F)$ is induced by a random variable $Y$ in $V$.

3. There exists a Gaussian measure $\mu$ on $\mathcal{B}(V)$ with covariance operator $R \in \mathcal{L}\left(V^{*}, V\right)$ such that

$$
\int_{0}^{T}\left\langle F(t), v^{*}\right\rangle^{2} d t=\left\langle R v^{*}, v^{*}\right\rangle
$$

for all $v^{*} \in V^{*}$.

4. $I_{F}$ is $\gamma$-radonifying.

For any (thus all) of the above we have

$$
E\left\|\int_{0}^{T} F(t) d B(t)\right\|^{2}=E\left\|\sum_{k=1}^{\infty} \gamma_{k} I_{F} f_{k}\right\|^{2} .
$$

Proof. See Theorem 2.3.4 in Riedle [2009a].

\subsubsection{Cylindrical Wiener Processes}

Let $\{W(t)\}_{t \geq 0}$ be a general cylindrical Wiener process in $U$. We have from Theorem 23.34 we can represent $W$ as

$$
W(t) U^{*}=\sum_{k=1}^{\infty}\left\langle i_{Q} e_{k}, u^{*}\right\rangle B_{k}(t),
$$


for all $u^{*} \in U^{*}$, where $\left\{e_{k}\right\}_{k \in \mathbb{N}}$ is an orthonormal basis of $H$ and $B_{k}(t)=W(t) u_{k}^{*}$ for $u^{*} \in U^{*}$ with $i_{q}^{*} u_{k}^{*}=e_{k}$.

A function $F:[0, T] \rightarrow \mathcal{L}(U, V)$ is in $M_{T}(U, V)$ if and only if

$$
t \mapsto\left[i_{Q}^{*} F^{*} v^{*}, h\right]
$$

is measurable for all $v^{*} \in V^{*}, h \in H$ and we have

$$
\int_{0}^{T}\left\|i_{Q}^{*} F^{*} v^{*}\right\|_{H}^{2}<\infty
$$

for all $v^{*} \in V^{*}$. We then say that $F$ is $V$-weakly in $L^{2}([0, T], H)$.

Definition 23.43. A function $F:[0, T] \rightarrow \mathcal{L}(U, V)$ is called stochastically Pettis integrable with respect to $W$ if it is $V$-weakly in $L^{2}([0, T], H)$ and there exists a $V$-valued random variable $Y$ such that for all $v^{*} \in V^{*}$

$$
I_{T}(F) v^{*}:=Z v^{*}:=\left\langle Y, v^{*}\right\rangle=\int_{0}^{T}\left\langle F(t) i_{q} e_{k}, v^{*}\right\rangle d B(t)
$$

a.s. and we denote

$$
Y=\int_{0}^{T} F(t) d W(t)
$$

Theorem 23.44. For a $V$-weakly $L^{2}([0, T])$ function $F:[0, T] \rightarrow V$ the following is equivalent:

1. F is stochastically Pettis integrable with respect to $W$;

2. the cylindrical integral $I_{T}(F)$ is induced by a random variable $Y$ in $V$.

3. there exists a Gaussian measure $\mu$ on $\mathcal{B}(V)$ with covariance operator $R \in \mathcal{L}\left(V^{*}, V\right)$ such that

$$
\int_{0}^{T}\left\|i_{Q}^{*} F^{*}(t) v^{*}\right\|_{H}^{2} d t=\left\langle R v^{*}, v^{*}\right\rangle
$$

for all $v^{*} \in V^{*}$;

4. $I_{F}$ is $\gamma$-radonifying.

Then we have that

$$
E\left\|\int_{0}^{T} F(t) d W(t)\right\|^{2}=E\left\|\sum_{k=1}^{\infty} \gamma_{k, l} I_{F}\left(f_{k} \otimes e_{l}\right)\right\|^{2}
$$

Proof. See Theorem 2.3.4 in Riedle [2009a]. 


\subsection{The Hilbert case}

\subsubsection{Nuclear and Hilbert-Schmidt Operators}

Let $V$ and $H$ be two separable Hilbert spaces and $\left\{e_{k}\right\}_{k \in \mathbb{N}}$ a complete orthonormal basis of $V$. A bounded linear operator $T: V \rightarrow H$ is called Hilbert-Schmidt if

$$
\sum_{k=1}^{\infty}\left\|T\left(e_{k}\right)\right\|_{H}^{2}<\infty
$$

This is property is independent of choice of basis. The set of Hilbert-Schmidt operators from $V$ into $H$ is denoted by $L_{2}(V, H)$ and the corresponding norm by

$$
\|T\|_{2}=\left(\sum_{k=1}^{\infty}\left\|T\left(e_{k}\right)\right\|_{H}^{2}\right)^{\frac{1}{2}} .
$$

With the following inner product

$$
\langle S, T\rangle_{2}=\sum_{k=1}^{\infty}\left\langle S\left(e_{k}\right), T\left(e_{k}\right)\right\rangle_{H},
$$

we have a Hilbert space. Note that for an operator $T \in L_{2}(V, H)$, then $T T^{*} \in L_{1}(H)$, where $T^{*}$ is the adjoint operator, and

$$
\|T\|_{2}^{2}=\operatorname{Tr}\left(T T^{*}\right)
$$

\subsubsection{Hilbert-valued Wiener Processes}

In the case of Hilbert spaces we do not need to take as much care as we do in the Banach case. Thus the theory is simpler and easier to grasp. We start by considering the stochastic integral w.r.t. a class of Hilbert-valued processes, the $Q$-Wiener process, which was introduced in Prato and Zabczyk [1992].

Assume $Q$ is a linear, symmetric, nonnegative definite and bounded operator on a Hilbert space, $H$, such that $\operatorname{Tr} Q<\infty$.

Definition 23.45. A $H$-valued stochastic process, $W$, is called a $Q$-Wiener process if

1. $W(0)=0$.

2. W has continuous trajectories.

3. $W$ has independent increments.

4. The law of $W_{t}-W_{S}$ is Gaussian with mean zero and covariance operator $(t-s) Q$ for all $0 \leq s \leq t$.

Let $\left\{e_{j}\right\}_{j \in \mathbb{N}}$ be an orthonormal basis of $H$ such that each $e_{j}$ is an eigenvalue of $Q$ and let $\lambda_{j}$ be the corresponding eigenvector. Further, let $\left\{\beta_{j}\right\}_{j \in \mathbb{N}}$ be a sequence of independent real-valued 
Brownian motions. Then the $H$-valued process

$$
W_{t}=\sum_{j=1}^{\infty} \sqrt{\lambda_{j}} \beta_{j}(t) e_{j}
$$

defines a $Q$-Wiener process, the convergence is in $L^{2}(\Omega, C([0, T, H))$.

\subsection{H-valued Integrals}

Let $V$ and $H$ be two separable Hilbert spaces. Let $V_{0}:=Q^{\frac{1}{2}}(V)$ and endow it with the following inner product:

$$
\langle h, g\rangle_{0}:=\left\langle Q^{\frac{1}{2}} h, Q^{\frac{1}{2}} g\right\rangle_{V}
$$

Let

$$
L_{2}^{0}:=L_{2}\left(V_{0}, H\right)
$$

be the Hilbert space of all Hilbert-Schmidt operators from $V_{0}$ into $H$ equipped with inner product

$$
\langle\Phi, \Psi\rangle_{L_{2}^{0}}=\sum: j=1^{\infty}\left\langle\Phi \tilde{e}_{j}, \Psi \tilde{e}_{j}\right\rangle_{H}
$$

where $\left\{\tilde{e}_{j}\right\}_{j \in \mathbb{N}}$ is an orthonormal basis of $V_{0}$. By using that

$$
\tilde{e}_{j}=\sqrt{\lambda_{j}} e_{j}=Q^{\frac{1}{2}}\left(e_{j}\right),
$$

where $\left\{e_{j}\right\}_{j \in \mathbb{N}}$ be an orthonormal basis of $H$ such that each $e_{j}$ is an eigenvalue of $Q$ and let $\lambda_{j}$ be the corresponding eigenvector. Then the norm of $\Psi \in L_{w}^{0}$ can be expressed as

$$
\|\Psi\|_{L_{2}^{0}}^{2}=\left\|\Psi \circ Q^{\frac{1}{2}}\right\|_{L_{2}(V, H)}^{2}=\operatorname{Tr}\left(\Psi Q \Psi^{*}\right) .
$$

Let $\Phi=\left\{\Phi_{t}\right\}_{t \in[0, T]}$ be a measurable $L_{2}^{0}$-valued process. Define the norm of $\Phi$ by

$$
\|\Phi\|_{T}:=\left(E\left[\int_{0}^{T}\|\Phi\|_{L_{2}^{0}}^{2} d s\right]\right)^{\frac{1}{2}} .
$$

Consider simple functions, i.e. finite linear combinations of process of the for $\mathrm{m} \Phi_{t}=\Phi_{0} \mathbf{1}_{(a, b]}$. Define for such functions the stochastic integral with values in $H$ as

$$
\int_{0}^{t} \Phi_{s} d W_{s}:=\sum_{j=1}^{n} \Phi_{j}\left(W_{b \wedge t}-W_{a \wedge t}\right)
$$

for $t \in[0, T]$. The map $\Phi \mapsto \int_{0}^{\cdot} \Phi_{s} d W_{s}$ is an isometry between the set of simple process and 
the space $M_{H}$ of square integrable $H$-valued $\mathcal{F}_{t}$-martingales endowed with the norm $\|X\|=$ $\left(E\left[\left\|X_{T}\right\|_{H}^{2}\right]\right)^{\frac{1}{2}}$. It follows from the standard way of proving the Itô-isometry for simple processes that we have

$$
E\left[\left\|\int_{0}^{T} \Phi_{t} d W_{t}\right\|_{H}^{2}\right]=\|\Phi\|_{T}^{2}
$$

Also as in the standard case, we extend this isometry to the linear space of the closure of the simple functions.

\subsection{The Real case}

We have defined the Hilbert space $V_{q}$ and the stochastic integral

$$
g \cdot W=\int_{0}^{T} g_{S} d W_{s}
$$

for a predictable stochastic process $g \in L^{2}\left(\Omega \times[0, T], V_{q}\right)$, such that we have the isometry

$$
E\left((g \cdot W)^{2}\right)=E\left[\left(\int_{0}^{T}\left\|g_{s}\right\|_{V_{q}}^{2} d s\right)^{2}\right] .
$$

For $s \in[0, T]$ and $g \in L^{2}\left(\Omega \times[0, T], V_{Q}\right)$, let us define $\Phi_{s}^{g}: V \rightarrow \mathbb{R}$ by

$$
\Phi_{s}^{g}(\eta):=\left\langle g_{s}, \eta\right\rangle_{V}
$$

for $\eta \in V$. Denote by $L_{2}^{0}$ the set $L_{2}\left(V_{0}, H\right)$ with $V_{0}=Q^{\frac{1}{2}}(V)$ and $H=\mathbb{R}$.

Proposition 23.46. $\Phi^{g}=\left\{\Phi_{s}^{g}\right\}_{s \in[0, T]}$ defines a predictable process with values in $L_{2}^{0}$ such that

$$
E\left[\int_{0}^{T}\left\|\Phi_{s}^{g}\right\|_{L_{2}^{0}}^{2} d W_{s}\right]=E\left[\int_{0}^{T}\left\|g_{s}\right\|_{V_{Q}}^{2} d s\right] .
$$

Therefore

$$
\int_{0}^{T} \Phi_{s}^{g} d W_{s}=\int_{0}^{T} g_{s} d s
$$

Proof. See proposition 3.5 in Dalang and Quer-Sardanyans [2010].

\subsection{The Cylindrical Case}

If $Q$ is a symmetric nonnegative definite and bounded operator on $V$, such that $\operatorname{Tr} Q \leq \infty$. Let $\left\{e_{j}\right\}_{j \in \mathbb{N}}$ be an orthonormal basis of $V$ such that each $e_{j}$ is an eigenvalue of $Q$ and let $\bar{\lambda}_{j}$ be 
the corresponding eigenvector. It is always possible to find a Hilbert space $V_{1}$ and a bounded linear injective operator $J: V \rightarrow V_{1}$ such that the restriction $J_{0}: V_{0} \rightarrow V_{1}$ is Hilbert-Schmidt.

\section{Proposition 23.47.}

1. Define $Q_{1}=J_{0} J_{o}^{*}: V_{1} \rightarrow V_{1}$. Then $Q_{1}$ is symmetric, nonnegative definite and $\operatorname{Tr} Q_{1}<\infty$.

2. Let $\tilde{e}_{j}=Q^{\frac{1}{2}}\left(e_{j}\right)$ and let $\left\{\beta_{j}\right\}$ be a family of independent real-valued Brownian motions. Then

$$
W_{t}:=\sum_{j=1}^{\infty} \beta_{j}(t) J_{0}\left(\tilde{e}_{j}\right),
$$

for $t \geq 0$, is a $Q_{1}$-Wiener process in $V_{1}$.

3. Let $I: V_{0} \rightarrow T_{0}^{*}$ be the one-to-one mapping which identifies $V_{0}$ with its dual $V_{0}^{*}$ and consider the following diagram:

$$
V_{1}^{*} \stackrel{\tilde{J}_{0}^{*}}{\rightarrow} V_{0}^{*} \stackrel{I^{-1}}{\rightarrow} V_{0} \stackrel{J_{0}}{\rightarrow} V_{1}
$$

Then for all $s, t \geq 0$ and $h_{1}, h_{2} \in V_{1}^{*}$,

$$
E\left[\left\langle h_{1}, W_{s}\right\rangle_{1}\left\langle h_{2}, W_{s}\right\rangle_{1}\right]=(t \wedge s)\left\langle\left(I^{-1} \circ \tilde{J}_{0}^{*}\right)\left(h_{1}\right),\left(I^{-1} \circ \tilde{J}_{0}^{*}\right)\left(h_{1}\right)\right\rangle_{V_{0}}
$$

4. $\operatorname{Im} Q_{1}^{\frac{1}{2}}=\operatorname{Im} J_{0}$ and

$$
\|h\|_{0}=\left\|Q^{-\frac{1}{2}} J_{0}(h)\right\|_{V_{1}}=\|\|_{Q^{\frac{1}{2}}\left(V_{1}\right)}
$$

where $Q^{-\frac{1}{2}}$ denotes the pseudo-inverse of $Q^{\frac{1}{2}}$.

Definition 23.48. For any square integrable predictable process $\Phi$ with values in $L_{2}^{=}$such that

$$
E\left[\int_{0}^{T}\left\|\Phi_{t}\right\|_{L_{2}^{0}}^{2} d t\right]<\infty,
$$

the $H$-valued stochastic integral $\Phi \cdot W$ is defined by

$$
\int_{0}^{T} \Phi_{s} d W_{s}=\int_{0}^{T} \Phi_{s} \circ J_{0}^{-1} d W_{s} .
$$

\subsection{Relation with the Random Field Approach}

Proposition 23.49. Let $\Phi=\left\{\Phi_{t}\right\}_{t \in[0, T]}$ be a predictable process in $L^{2}\left(\Omega \times[0, T], L_{2}\left(V_{0}, H\right)\right)$, so the we can define the $H$-valued stochastic integral. Let $g^{\Phi}=\left\{g_{t}^{\Phi}\right\}_{t \in[0, T]}$ be the predictable 
process in $\left.L^{2}\left(\Omega \times[0, T], V_{Q} \otimes H\right)\right)$ which is associated to $\Phi$. That is, for all $t \in[0, T]$,

$$
g_{t}^{\Phi}=\sum_{j, k=1}^{\infty}\left(g_{t}^{\Phi}\right)^{j, k}\left(v_{j} \otimes f_{k}\right)
$$

where $\left(g_{t}^{\Phi}\right)^{j, k}=\left\langle\Phi_{s}\left(\tilde{e}_{j}\right), f_{k}\right\rangle_{H}$.

Then the $H$-valued stochastic integral $\int_{0}^{T} g_{t}^{\Phi} d W_{t}$ is well defined and

$$
\int_{0}^{T} g_{t}^{\Phi} d W_{t}=\int_{0}^{T} \Phi_{t}^{g} d W_{t}
$$

Proof. See proposition 3.12 in Dalang and Quer-Sardanyans [2010].

\subsection{Lèvy Processes in Banach spaces}

Let $\varrho:=d t \otimes \nu$.

Definition 23.50. A function $F:[0, T] \times B \rightarrow V$ is called stochastically Pettis integrable with respect to $\tilde{N}$ if it is $V$-weakly $L^{2}([0, T] \times B, \varrho)$ and there exists a $V$-valued random variable $Y$ such that for all $v^{*} \in V^{*}$

$$
Z v^{*}:=\left\langle Y, v^{*}\right\rangle=\int_{[0, T] \times B}\left\langle F(t, u), v^{*}\right\rangle \tilde{N}(d t, d u),
$$

a.s. and we denote

$$
Y=\int_{[0, T] \times B} F(t) \tilde{N}(d t, d u) .
$$

Definition 23.51. An adapted cylindrical process $L=\left\{L_{t}\right\}_{t \geq 0}$ in $U$ is a cylindrical Lévy process if for all $u_{1}^{*}, \ldots, u_{n}^{*} \in U^{*}$ and $n \in \mathbb{N}$ the $\mathbb{R}^{n}$-valued stochastic process

$$
\left\{L / t\left(u_{1}^{*}, \ldots, L(t) u_{n}^{*}\right\}_{t \geq 0}\right.
$$

is a Lévy process.

Let us identify $H$ with $l^{2}$. From parseval's identity we have

$$
x=\sum\left\langle x, e_{k}\right\rangle e_{k}=\sum x_{k} e_{k}
$$

such that we have an isomorphism $\gamma$ between $H$ and $l^{2}$ defined by

$$
x \in H \mapsto \gamma(x)=\left(x_{k}\right)_{k} \in l^{2} .
$$


Now, let $H_{n}$ be the projection of $H$ to the $n$-dimensional subspace and

$$
U(t, x)=\sum_{k}\left\langle U(t, x), e_{k}\right\rangle e_{k}=\sum_{k} u_{k}(t, x) e_{k}
$$

for $U:[0, T] \times H \rightarrow H$. Assume that we have a family $\left\{L_{n}\right\}_{n \geq 0}$ of independent real-valued Lévy process with Lévy-Khintchine representation

$$
E\left[e^{i \zeta L_{n}(t)}\right]=e^{-t \Psi_{n}(\zeta)}, t \geq 0, \zeta \in \mathbb{R}
$$

The following theorem tells us when a family of Lévy processes,

$$
L:=\left(\sum L_{n}(t) e_{n}\right)_{t \geq 0},
$$

gives us a Hilbert (or even more general Banach (U) ) valued process.

Theorem 23.52 (Theorem 4.40 in Peszat and Zabczyk [2007]).

1. Assume that a U-valued Lévy process is given by (23.5) with independent Lévy processes $L_{n}$ having representation (23.4). Then

$$
\sum_{n} \int_{\mathbb{R}}\left(|z|^{2} \wedge 1\right) v_{n}(d z)<\infty .
$$

Moreover, $L$ is square integrable if and only if

$$
\sum_{n} \int_{\mathbb{R}}|z|^{2} v_{n}(d z)<\infty .
$$

2. Conversely, if (23.4) and (23.6) hold and the $L_{n}$ are independent Lévy processes, then (23.5) defines a Lévy process on $U$. Moreover, the series in (23.5) converges, $\mathbb{P}-a . s$. , uniformly in $t$ on any compact interval.

Proof. See Theorem 4.40 in Peszat and Zabczyk [2007]. 


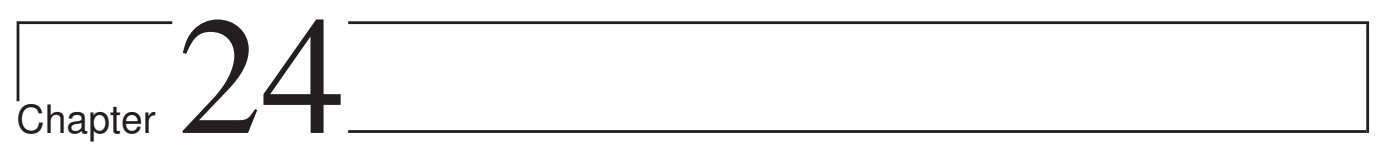

\section{Manifolds and Lie Groups}

This chapter is based on Lejay [2009] and Hsu [2002].

A m-dimensional smooth manifold is a set $M$ such that for each point $p \in M$ there exists open set $U_{p} \subset \mathbb{R}^{m}$ and an open neighbourhood $V_{p}$ of $p$ such that it exists a map $\Phi_{p} \mathrm{~m}$ which is a homeomorphism from $U_{p}$ to $V_{p} \cap M$. Further, for $p, q \in M$ such that $V_{\cap} V_{q} \neq \emptyset$ we have that $\Phi_{p} \circ \Phi_{q}^{-1}$ and $\Phi_{q} \circ \Phi_{p}^{-1}$ are smooth on their domains. Given a manifold $M$, denote by $T_{p} M$ the tangent space of $M$ at $p$ and $T M=\cup_{p \in M} T_{p} M$. A integral curve along $X$ is a smooth path $\gamma:[0, T] \rightarrow M$ such that $\gamma^{\prime}(t)=X_{\gamma(t)}$.

\subsection{Matrix Groups}

Let $M$ be a matrix group. That is a subset of $d \times d$-matrices such that for $p, g \in M$ we have that $p \times q \in M, p^{-1} \in M$ and $M$ is closed. A matrix group forms a smooth manifold. Let us define two smooth maps: for $p \in M$ let

$$
R_{p}(q)=q \times p
$$

and

$$
L_{p}(q)=p \times q
$$

for all $q \in M$. Then the differentials are given by

$$
d_{q} R_{p}(v)=v \times p
$$

and

$$
d_{q} L_{p}(v)=p \times v
$$

for all $q \in M$ and $v \in T_{q} M$. A left-invariant vector field is a vector field $X$ such that $d_{q} L_{p}\left(X_{q}\right)=$ $X_{L_{p}(q)}$. Let $\gamma$ be the integral curve of a left-invariant vector field $X$ with $\gamma(0)=p$. Then

$$
\gamma^{\prime}(t)=X_{\gamma(t)}=\gamma(t) \times X_{I d}=\gamma(t) \times p^{-1} \times X_{p} .
$$


For $p=I d$ and $X_{I d}=v$ we have that $\gamma^{\prime}(t)=\gamma(t) \times v$ such that by solving this we get

$$
\gamma(t)=\exp (t v)
$$

where exp is the matrix exponential

$$
\exp (v)=I d+\sum_{k=1}^{\infty} \frac{1}{k !} v^{k} .
$$

\subsection{Lie groups and algebras}

A group $(G, \times)$ that is such that $(x, y) \mapsto x \times y$ and $x \mapsto x^{-1}$ are of class continuous is called a Lie group.

Definition 24.1. A Lie algebra is a vector space $\mathfrak{g}$ over some field $F$ with a binary operation $[\cdot, \cdot]: \mathfrak{g} \times \mathfrak{g} \rightarrow \mathfrak{g}$ called the Lie bracket, which satisfies the following axioms:

1. Bilinearity:

$$
[a x+b y, z]=a[x, z]+b[y, z], \quad[z, a x+b y]=a[z, x]+b[z, y]
$$

for all scalars $a, b$ in $F$ and all elements $x, y, z$ in $\mathfrak{g}$.

2. Alternating on $\mathfrak{g}$ :

$$
[x, x]=0
$$

for all $x \in \mathfrak{g}$.

3. The Jacobi identity:

$$
[x,[y, z]]+[z,[x, y]]+[y,[z, x]]=0
$$

for all $x \in \mathfrak{g}$.

Lie's fundamental theorems describe a relation between Lie groups and Lie algebras. In particular, any Lie group gives rise to a canonically Lie algebra (the tangent space at the identity), and conversely, for any Lie algebra there is a corresponding connected Lie group. Let $L$ denote a smooth second order elliptic, not necessarily nondegenerate, differential operator on a differentiable manifold $M$.

\subsection{Martingales on Manifolds and Lie groups}

Definition 24.2. Let $\hat{M}=M \cup \delta_{M}$ be the one point compactification of $M$. An $M$-valued path $x$ with explosion time $e(x)>0$ is a continuous map $x:[0, \infty) \rightarrow \hat{M}$ such that $x_{t} \in M$ for $0 \leq t<e$ and $x_{t}=\delta_{M}$ for all $t \geq e$ if $e<\infty$. The space of all $M$-valued path with explosion time is called the path space of $\bar{M}$ and is denoted by $W(M)$.

We now define $M$-valued semimartingales. 
Definition 24.3. Let $\tau$ be an $\mathcal{F}_{*}$-stopping time. A continuous, $M$-valued process $X$ defined on $[0, \tau)$ is called an $M$-valued semimartingale if $f(X)$ is a real-valued semimartingale on $[0, \tau)$ for all $f \in C^{\infty}(M)$, 


\section{References}

K. Aase, B. Øksendal, N. Privault, and J. Ubøe. White noise generalizations of the ClarkHaussmann-Ocone theorem with application to mathematical finance. Finance Stoch., 4: 465-496, 2000. 114

C. Acerbi. Coherent representations of subjective risk-aversion. In:G. Szegö (Ed.). Risk measures for the 21st century. Wiley, pages 147-207, 2004. 207

R. A. Adams and J. J. F. Fournier. Sobolev Spaces. Elsevier, 2nd edition, 2003. 137, 150, 153, 228

N. Agram, S. Haadem, B. Øksendal, and F. Proske. A maximum principle for infinite horizon delay equations. To be published in SIAM journal on Mathematical Analysis, 2013. 54, 107

T. T. K. An and B. Øksendal. A maximum principle for stochastic differential games with partial information. J. of Optim. Theory and Appl, 139:463-483, 2008. 38

T. T. K. An, B. Øksendal, and Y. Y. Okur. A malliavin calculus approach to general stochastic differential games with partial information. Eprint, Univ. of Oslo, 26, 2008. 38

D. Applebaum. Lévy Processes and Stochastic Calculus. Cambridge, 2 edition, 2009. 140, 141, $146,183,185,214,215,220$

P. Artzner, F. Delbaen, J.M Eber, and D. Heath. Thinking coherently. Risk, 10:68-71, 1997. 202, 203, 205

P. Artzner, F. Delbaen, J.M Eber, and D. Heath. Coherent measures of risk. Mathematical Finance, 3:203-228, 1999. 202

F. Baghery and B. Øksendal. A maximum principle for stochastic control with partial information. Stochastic Analysis and Applications, 25(3):705-717, 2007. 54

F. Baghery, Y. Hu, B. Øksendal, and T. Zhang. Stochastic Calculus for Fractional Brownian Motion and Applications. Springer, 2008. 14, 107, 108, 110, 113, 114, 124, 125, 249, 250

G. Barles, R. Buckdahn, and E. Pardoux. Backward stochastic differential equations and integral-partial differential equations. Stochastics and Stochastics Reports, 60:57-83, 2009. $14,56,78,117$

R. F. Bass. Uniqueness in law for pure jump markov processes. Probab. Theory Related Fields, 79:271-287, 1988. 133

R. F. Bass and Z.-Q. Chen. Systems of equations driven by stable processes. Probab. Theory Related Fields, 134:175-214, 2006. 133

R. F. Bass, K. Burdzy, and Z.-Q. Chen. Stochastic differential equations driven by stable processes for which pathwise uniqueness fails. Stoch. Proc. and their Appl., 3(1):1-15, 2004. 132 
R.F. Bass. Stochastic differential equations with jumps. Probability Surveys, 1:1-19, 2004. 132

C. Bender. An Itō formula for generalized functions of a fractional brownina motion with arbitrary Hurst parameter. Stochastic Processes and their Applications, 104:81-106, 2003. 107,249

A. Bensoussan and A. Friedman. Nonlinear variational inequalities and differential games with stopping times. J. Funct. Anal, 16:305-352, 1974. 43

Y. M. Berezansky and Y. G. Kondratiev. Spectral Methods in Infinite-Dimensional Analysis. Kluwer Academic Publishers, 1995. 108

J. Bertoin. Lévy Processes. Cambridge University Press, 4th edition, 2005. 132, 134, 135, 183, 184

F. Biagini, Y. Hu, B. Øksendal, and A. Sulem. A stochastic maximum principle for processes driven by fractional Brownian motion. Stochastic Processes and their Applications, 100: 233-253, 2002. 107, 128

I. H. Biswas. On zero-sum stochastic differential games with jump-diffusion driven state: a viscosity solution framework. Submitted to SIAM J. Control and Opt. 38, 39, 41

N. Bouleau and F. Hirsch. Dirichlet Forms abd Analysis on Wiener Space. deGruyter, 1991. 224

A. Brown and D. Einhorn. Private profits and socialized risk. GARP Risk Review, 42, 2008. URL http://www.garpdigitallibrary .org/download/GRR/2012.pdf . 201

R. Buckdahn, J. Ma, and C. Rainer. Stochastic control problems for systems driven by normal martingales. Ann. Appl. Probab, 18(2):632-663, 2008. 39

C.Bender, T. Sottinen, and E. Valkeila. Arbitrage with fractional Brownian motion? Theory of Stochastic Processes, 12(28), 2006. 2

L. Chen and Z. Wu. Maximum principle for the stochastic optimal control problem with delay and application. Automatica, 46:1074-1080, 2010. 78

P. Cheridito. Arbitrage in fractional Brownian motion models. Finance and Stochastics, 7(4): 533-553, 2003. 2

R. Cont and P. Tankov. Financial modelling with jump processes. Chapman \& Hall/CRC, 2004. 53, 207

L. Coutin. An introduction to (stochastic) calculus with respect to fractional brownin motion. 250

L. Coutin and Z. Qian. Stochastic analysis, rough path analysis and fractional brownin motions. PTRF, 122:108-140, 2002. 257

R. C. Dalang and L. Quer-Sardanyans. Stochastic integrals for spde's: A comparison. 2010. doi: 10.1016/j.exmath.2010.09.005. URL http://dx.doi.org/10.1016/j.exmath. 2010 . 09.005. 258, 259,260,273, 275

F. Delbaen. Coherent measure of risk on general probability spaces. Advances in Financeand Stochastics, pages 1-37, 2002. 205

I. Elsanosi, B. Øksendal, and A. Sulem. Some solvable stochastic control problems with delay. Stochastics and Stochastics Reports, 71:69-89, 2000. 78, 85

L.C. Evans and P.E. Sougadinis. Differential games and representation formulas solutions for hamilton-jacobi-isaacs equations. Indiana University Mathematics Journal, 33(5):773-797, 1984. 42 
F. Flandoli. Random Perturbation of PDEs an Fluid Dynamic Models. Springer, 2010. 148, 163

F. Flandoli, M. Gubinelli, and E. Priola. Well-posedness of the transport equation by stochastic perturbation. Invent. Math., 180:1-53, 2010. 133

F. Flandoli, T. Nilssen, and F. Proske. Malliavin differentiability and strong solutions for a class of sde's in hilbert spaces. Manuscipt, 2012. 132

W. H. Fleming and P.E. Souganidis. On the existence of value functions of two-player, zero-sum stochastic differential games. Indiana University Mathematics Journal, 38(2), 1989. 38

H. Föllmer and P. Leukert. Quantile hedging. Finance and Stochatics, 3:251-273, 1999. 203, 208

H. Föllmer and I. Penner. Back to the future. Statistics \& Decisions, 24:061 - 096, 2006a. 206

H. Föllmer and I. Penner. Convex risk measures and the dynamics of their penalty functions. Statistics \& Decisions, 24:061 - 096, 2006b. 204, 208

H. Föllmer and A. Schied. Convex measures of risk and trading constraints. Finance Stochast, 6:449-477, 2002. 3, 24, 34

H. Föllmer and A. Schied. Robust preferences and convex risk measures. In:Advances in Finance and Stochastics, Essays in Honor of Dieter Sondermann, K. Sandmann and J. Schonbucher, eds. Springer Nwe york, pages 39-56, 2002. 206, 207

M. Frittelli and E. R. Gianin. Dynamic convex risk measures. In:G. Szegö (Ed.). Risk measures for the 21st century. Wiley, 3:227-248, 2004. 208

M. Frittelli and E. Rosazza Gianin. Putting order in risk measures. J. Banking \& Finance, 26: 1473-1486, 2002. 3, 24, 34, 203, 206, 208

P. K. Friz and N. B. Victoir. Multidimensional Stochastic Processes as Rough Paths. Cambridge, 1 edition, 2010. 168

M. Fukushima, Y. Oshida, and M. Takada. Dirichlet Forms and Symmetric Markov Processes. Berlin; New York: de Gruyter, 1994. 220, 224

H. Füllmer and A. Schied. Convex measures of risk and trading constraints. Finance and Stochastic's, 5:429-447, 2002. 203

E. R. Gianin. Some examples of risk measures via g-expectations. Working paper no. 41 july, Università di Milano Bicocca, Italy, 2002a. 208, 216, 219

E. R. Gianin. Convexity and law invariance of risk measures. PhD Thesis, Università di Bergamo, Italy, 2002b. 208

P. Grandits and T. Rheinlander. On the minimal entropy martingale measure. The annals of probability, 30:1003-1038, 2002. 207

I. Gyöngy and N. V. Krylov. Existence of strong solutions for itô's stochastic equations via approximations. Probab. Theory Relat. Fields, 105:143-158, 1996. 132

I. Gyöngy and T. Martinez. On stochastic differential equations with locally unbounded drift. Czechoslovak Mathematical Journal, 51(4):763-783, 2001. 132

S. Haadem. Risk measures and differential games. Duo, 2009. URL https://www.duo.uio. no/handle/10852/10828?locale-attribute=en. 201, 216

S. Haadem, S. Mohammed, B. Øksendal, and F. Proske. Time-advanced backward spde's and optimal control of spde's with delay and partial information. Preliminary version, 2012. 107 
S. Haadem, B. Øksendal, and F. Proske. Maximum principles for jump diffusion processes with infinite horizon. Automatica, 2013. doi: 10.1016/j.automatica.2013.04.011. URL http: //dx.doi.org/10.1016/j.automatica.2013.04.011. 14, 78, 97, 107, 117, 125

H. Halkin. Necessary conditions for optimal control problems with infinite horizons. Econometrica, 42:267-272, 1974. 2, 53, 62, 78, 107

U. Haussman. A stochastic maximum priciple for optimal control of difusions. Longman scientific and technical, 1986. 125

T. Hida, H.Kuo, J. Potthoff, and L. Streit. White Noise: An Infinite Dimensional Calculus. Springer, 1993. 108, 240

R. Hilfer. Threefold introduction to fractional derivatives. In R. Klages et al., editor, Anomalous Transport: Foundation and Applications. Wilet-VCH, Weinheim, 2008. 246

H. Holden, B. Øksendal, J. Ubøe, and T. Zhang. Stochastic partial differential equations: A modeling, white noise functional approach. Springer, second edition, 2010. 108, 109, 240, 244,255

E. P. Hsu. Stochastic Analysis on Manifolds. AMS, 2002. 277

Y. Hu and B. Øksendal. Fractional white noise calculus and applications to finance. Infin. Dimens. Anal. Quantum Probab. Relat. Top., 6:1-32, 2003. 115, 129

Y. Hu and S. Peng. Backward stochastic differential equation driven by fractional Brownian motion. SIAM J. Control Optim, 48(3):1675-1700, 2009. 107, 115, 117, 120, 121

Z. Hu and D. Nualart. Rough path analysis wia fractional calculus. Transactions of the American mathematical society, 361(5):2689-2718, 2009. 167, 171

Y. Ishikawa and H. Kunita. Malliavin calculus on the wiener-poisson space and its. application to canonical sde with jumps. Stoch. Proc. and Appl, 116(12):1743-1769, 2006. 39

J. Jacod and A. Shiryaev. Limit Theorems for Stochastic Processes. Springer-Verlag Berlin, 2nd edition, 2003. 25, 27

S. Jaschke and U. Küchler. Coherent risk measures and good-deal bounds. Finance and Stochatics, 5:181-200, 2001. 202

J. Jia and S. Dyer. A standard measure of risk and risk-value models. Management Science, 42:1691-1705, 1996. 203

P. Jorion and N. Taleb. The jorion-taleb debate. Derivatives Strategy, 1997. URL http: //www. derivativesstrategy . com/magazine/archive/1997/0497fea2. asp. 201

G. Kallianpur and J. Xiong. Stochastic Differential Equations in Infinite Dimensional Spaces. IMS Lecture Notes Monograph Series, 1995. 135, 158, 238

I. Karatzas and S. E. Shreve. Brownian Motion and Stochstic Calculus. Springer-Verlag, New York, 2nd edition, 2000. 173, 175, 178, 179, 180, 181, 182, 213

T. Kato. Perturbation Theory for Linear Operators. Springer, 1 edition, 1995. 152

P. Kim and R. Song. Potential theory of truncated stable processes. Math. Z., 256(1):139-173, 2007. 141

M. L. Kleptsyna. On strong solutions of stochastic equations with degenerate coefficients. Theory of Probab. Appl., 29:403-407, 1984. 132

V. I. Kolyada. Estimates of fourier transform in sobolev spaces. Studia Mathematica, 125(1), 1997. 141 
N. V. Krylov. Controlled Diffusion Processes. Springer, 1980. 29, 38, 44, 45, 46, 48, 50, 132, $146,147,157$

N. V. Krylov and M. Röckner. Strong solutions of stochastic equations with singular time dependent drift. Prob. Theory Rel. Fields, 131(2):154-196, 2005. 132

H. Kuo. White noise distribution theory. CRC Press, 1996. 108, 240

A. Lejay. Yet another introduction to rough paths. Sèminaire de probabilitès, pages 1-101, 2009. $168,230,232,233,234,277$

R. T. Lewis. Singular elliptic operators of second order with purely discrete spectra. Transactions of the American mathematical society, 271(2):653-666, 1982. 152

J. Li and S. Peng. Stochastic optimization theory of backward stochastic differential equations with jumps and viscosity solutions of Hamilton-Jacobi-Bellman equations. Nonlinear Analysis, 70:1779-1796, 2009. 14, 56, 78, 117

X. Li and S. Tang. Necessary conditions for optimal control of stochastic systems with random jumps. SIAM Journal of Control and Optimization, 32:1447-1475, 1994. 14, 56, 117

A. Lunardi. Interpolation theory. Scuala Normale Superiore di Pisa (New Series), Edizioni della Normale, Pisa, 2, 2009. 146

H. Lüthi and J. Doege. Convex risk measures for portfolio optimization and concepts of flexibility. Mathematical Programming, 104:541-559, 2005. 207

J. Ma and J. Yong. Forward-Backward Stochastic Differential Equations and their Applications. Springer, 2007. 216, 217, 219

B. B. Mandelbrot and J.W. Van Ness. Fractional brownian motion, fractional noise and applications. SIAM Review, 10:422-437, 1968. 250

B. Maslowski and P. Veverka. Infinite horizon maxmimum principle for the discounted control problem - incomplete version. arXiv, 2011. 53, 78

S. Mataramvura and B. Øksendal. Risk minimizing portfolios and hjbi equations for stochastic differential games. Stochastics, 80(4):317-337, 2008. 24

R. E. Megginson. An introduction to Banach space theory. New York: Springer, 1998. 260

O. Menoukeu-Pamen, T. Meyer-Brandis, T. Nilssen, F. Proske, and T. Zhang. A variational approach to the construction and malliavin differentiability of strong solutions of sde's. Preprint, University of Oslo, 2011. 132, 133

T. Meyer-Brandis and F. Proske. Construction of strong solutions of sde's via malliavin calculus. Journal of Funct. Anal., 258:3922-3953, 2010. 132, 133

I. Mitoma. Tightness of probabilities in $\mathrm{C}\left([0,1], \mathrm{S}^{\prime}\right)$ and $\mathrm{D}\left([0,1], \mathrm{S}^{\prime}\right)$. Annals. of Probability, 11 (4):989-999, 1983. 156

S.-E. A. Mohammed. Stochastic differential systems with memory. Spring School on Stochastic Delay Differential Equations, 2003. 225, 227

S.-E. A. Mohammed. Stochastic differential systems with memory: Theory, examples and applications. Articles and Preprints, Paper 50, 1998. 226, 227

S.-E.A. Mohammed. Lecture notes in mat4760. UiO, 2011. 225

S.-E.A. Mohammed and M.K.R. Scheutzow. Lyapunov exponents of linear stochastic functional differential equations driven by semimartingales, part ii: Examples and case studies. Annals of Probability, 25(3):1210 - 1240, 1997. 78, 92 
J. Nadal. Dynamic risk measures: Time consistency and risk measures from BMO martingales. Finacial stochastics, 12:219-244, 2008. 209

E. D. Nezza, G. Palatucci, and E. Valdinoci. Hitchiker's guide to the fractional Sobolev spaces, 2011. 153

D. Nualart. The Malliavin Calculus and Related Topics. Springer, 2006. 133, 135, 160, 236, $237,249,250$

G. D. Nunno, Bernt Øksendal, and Frank Proske. Malliavin Calculus for Lèvy Processes with Applications to Finance. Springer, 2009. 133, 134, 135, 153, 157, 183, 236

N. Obata. White noise calculus and Fock space. Springer, 1994. 108, 240

B. Øksendal. Stochastic Differential Equations: An Introduction with Applications. Springer, fourth edition, 2007. 173

B. Øksendal and A. Sulem. A Maximum Principle for Optimal Control of Stochastic Systems with Delay, with Applications to Finance: In J.M. Menaldi, E. Rofman and A. Sulem (editors): Optiaml Control and Partial Differential equations - Innovations and Applications. IOS Press, Amsterdam, 2000. 187, 191, 198, 254, 256

B. Øksendal and A. Sulem. Applied Stochastic Control of Jump Diffusions. Springer-Verlag Berlin, 2nd edition, 2007. 24, 26, 27, 78, 85, 90

B. Øksendal and A. Sulem. A game theoretic approach to martingale measures in incomplete markets. Surveys of Applied and Industrial Mathematics, 15:18-24, 2008. 38, 78

B. Øksendal and T. Zhang. On backwards stochastic differential equations. Preprint,UiO, 2001. 216,217

B. Øksendal, A. Sulem, and T. Zhang. Optimal control of stochastic delay equations and timeadvanced backward stochastic differential equations. Adv. Appl. Prob., 43:572-596, 2011. 78, 81

J. M. Eber P. Artzner, F. Delbaen and D. Heath. Coherent measures of risk. Mathematical Finance, 4:203-228, 1999. 34

E. Pardoux. Bsdes', weak convergence and homogenizations of semilinear pdes. In F.H. Clark and R.J. Stern, editors, Nonlinear Analysis, Differential Equations and Control, pages 503549. Kluwer Academic, Dordrecht, 1999. 14, 53, 56, 78, 117

E. Pardoux and S. Peng. Adapted solution of a backward stochastic differential equation. Syst. Control Lett, 14:55-61, 1990. 107

G. Pedersen. Analysis Now. Springer, 1995. 204

S. Peng. G-expectation, G-Brownian motion and related stochastic calculus of Itô type. arXiv:math/0601035, 2006. 209

S. Peng. G-Brownian motion and dynamic risk measure under volatility uncertainty. arXiv:0711.2834, 2007. 209

S. Peng. Backward sde and related g-expectations. In: Backward Stochastic Differential Equations, N. El Karoui and L. Mazliak (ed)Pitman REs. Notes Math. Ser., 364:141-159, 1997. 208, 209

S. Peng. Nonlinear expectations and risk measures. Lectures to be presented in CIME, July 7-12, 2003 Bressanone, Italy, 2003. 208

S. Peng and Y. Shi. Infinite horizon forward-backward stochastic differential equations. Stoch. Proc. and their Appl., 85:75-92, 2000. 14, 54, 56, 78, 117, 125, 216, 219 
S. Peszat and J. Zabczyk. Stochastic Partial Differential Equations with Lévy Noise. Cambridge, 1 edition, 2007. 276

H. Pham. Optimal stopping of controlled jump diffusion processes. a viscosity solution approach. Journal of Mathematical Systems, Estimations and Control, 8(1):1-27, 1998. 29, 38

V. Pipiras and M. S. Taqqu. Integral questions related to fractional brownina motion. Probability Theory Related Fields, 118:251-291, 2000. 248

J. Potthoff and M. Timpel. On a dual pair of smooth and generalized random variables. Potential Analysis, 4:637-654, 1995. 114

G. Pragarauskas and P. A. Zanzotto. On one-dimensional stochastic differential equations with respect to stable processes. Lithuanian Math. J., 40(3):277-295, 2000. 133

G. D. Prato, P. Malliavin, and D. Nualart. Compact famies of Wiener functionals. C.R. Acad. Sci. Paris, 315:1287-1291, 1992. 138

G. Da Prato and J. Zabczyk. Stochastic equations in infinite dimensions. Cambridge University Press, 1992. 271

G. Da Prato, F. Flandoli, E. Priola, and M. Röckner. Strong uniqueness for stochastic evolution equations in hilbert spaces with bounded and measurable drift. arXiv, 1109.0363v1, 2011. 132

E. Priola. Pathwise uniqueness for singular sde's driven by stable processes. Osaka J. of Math., 49(2):421-447, 2012. 132, 133

P. Protter. Stochastic Integration and Differential Equations. Springer, 2nd edition, 2005. 132

F. Riedel. Dynamic coherent risk measires, working paper. http://www.wiwi.hu-berlin.de/ riedel, 2003. 203

M. Riedle. Stochastic processes in Banach spaces. The University of Manchester, 2009a. 258, $262,263,266,267,268,269,270$

M. Riedle. Cylindrical wiener processes. Sèminaire de provailitès, Berlin:Springer. Lecture Notes in Mathematics, 2009b. 266

L. C. G. Rogers and D. Williams. Diffusions, Markov Processes, and Martingales. Volume Two; Itô Calculus. Cambridge University Press, second edition, 2000a. 173

L. C. G. Rogers and D. Williams. Diffusions, Markov Processes, and Martingales. Volume One: Foundations. Cambridge University Press, second edition, 2000b. 54, 173, 175

M. Royer. Backward stochastic differential equations with jumps and related non-linear expectations. Stochastic Processes and Their Applications, 116:1358-1376, 2006. 14, 56, 70, 78, 117

W. Rudin. Functional Analysis. McGraw-Hill, 2nd edition, 1991. 220, 240, 259

A. Seierstad, A. Strøm, and K. Sydsæter. Matematisk Analyse (In Norwegian). Gyldendal Akademisk, 4 edition, 2006. 54

R. Situ. On solutions of backward stochastic differential equations with jumps and with nonLipschitzian coefficients in Hilbert spaces and stochastic control. Statistics and Probability Letters, 60:279-288, 2002. 14, 56, 78, 117

A. Sulem and B. Øksendal. Applied Stochastic Control of Jump Diffusions. Springer, second edition, 2007. 54, 193, 194, 199, 200

H. Tanaka, M. Tsuchiya, and S. Watanabe. Perturbation of drift-type for Lévy processes. J. Math. Kyoto Univ., 14:73-92, 1974. 132 
J. van Nerven. Stochastic Evolution Equations. 2008. 258, 265

A. Černý and S. Hodges. The theory of good-deal pricing in financial markets. Imperial College Management School, London, 2000. 202

A. Y. Veretennikov. On the strong solutions of stochastic differential equations. Theory Probab. Appl., 24:354-366, 1979. 132

J. Yin. On solutions of a class of infinite horizon FBSDEs. Statistics and Probability Letters, 78:2412-2419, 2008. 14, 56, 78, 117

J. Yong and X. Y. Zhou. Stochastic Controls: Hamiltonian Sytems and HJB Equations. Springer, 1999. 125, 187, 189, 192, 196

L. C. Young. An inequality of the Hölder type, connected with Stieltjes integration. Acta Math., 67:251-282, 1936. 232, 256

M. Zähle. Integration with respcet to fractal functions and stochastic calculus. Probablity Theory and Related Fields, 111(3):333-374, 1998. 162, 257

P. A. Zanzotto. On stochastic differential equations driven by a cauchy process and other stable Lévy motions. Ann. Probab., 30(2):802-825, 2002. 133

T. Zhang. Lecture notes in mat4760. UiO, 2009. 216, 217

A. K. Zvonkin. A transformation of the state space of a diffusion process that removes the drift. Math.USSR (Sbornik), 22:129-149, 1974. 3, 132 
